\title{
Simulación de la transferencia de energía en hornos monoestrato de rodillos para la cocción de baldosas cerámicas
}

\author{
Memoria que para optar al grado de \\ Doctor Ingeniero Químico \\ presenta
}

Alberto Escrig Vidal

Castelló, 2017

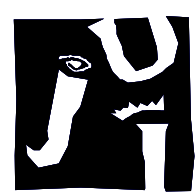

UNIVERSITAT JAUME I

Escola Superior de Tecnologia i Ciències Experimentals

Departament d'Enginyeria Química 

Eliseo Monfort Gimeno, Catedrático de Ingeniería Química de la Universitat Jaume I de Castelló, y Vicent Cantavella Soler, Doctor Ingeniero Químico,

CERTIFICAN: que AlBerto EscRig Vidal ha realizado bajo su dirección, dentro del programa de doctorado «Tecnologies Industrials, Materials i Edificació» de la Universitat Jaume I de Castelló, el trabajo que bajo el título «Simulación de la transferencia de energía en hornos monoestrato de rodillos para la cocción de baldosas cerámicas» presenta en esta Memoria y que constituye su Tesis para optar al grado de Doctor Ingeniero Químico. 



\section{Índice general}

1. Introducción $\quad \mathbf{1}$

1.1. Tipos de baldosas cerámicas y proceso de fabricación . . . . . . . . . . . . 1

1.2. Consumo de energía en la fabricación de baldosas cerámicas . . . . . . . . . 4

1.3. Cocción de baldosas cerámicas . . . . . . . . . . . . . . . 5

1.4. Hornos monoestrato de rodillos . . . . . . . . . . . . . . . . . 7

1.5. Mecanismos de transferencia de energía en hornos de rodillos . . . . . . . . 17

1.6. El método zonal . . . . . . . . . . . . . . . . . . . . . . 22

2. Motivación, objetivos y alcance $\quad 29$

2.1. Motivación ........................ . . 29

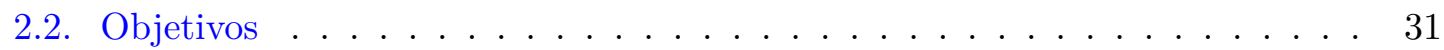

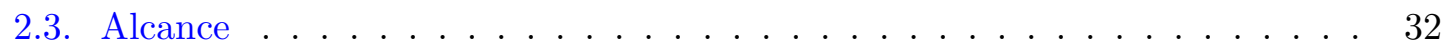

3. Modelo propuesto $\quad 33$

3.1. Planteamiento general. Hipótesis y simplificaciones . . . . . . . . . . . . . 33

3.2. Áreas de intercambio directo . . . . . . . . . . . . . . . . . . 37

3.3. Balances de energía . . . . . . . . . . . . . . . . . . 57

3.4. Resolución de los balances de energía . . . . . . . . . . . . . . . . . . . 66

3.5. Cálculo de la regulación del horno . . . . . . . . . . . . . . . 72

$\begin{array}{ll}\text { 4. Validación del modelo } & \mathbf{7 7}\end{array}$

4.1. Experimental . . . . . . . . . . . . . . . . . . . 77

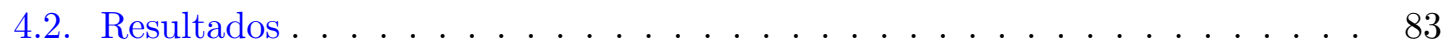

$\begin{array}{lr}\text { 5. Aplicación del modelo } & \mathbf{9 7}\end{array}$

5.1. Caso de estudio . . . . . . . . . . . . . . . . . . . 97

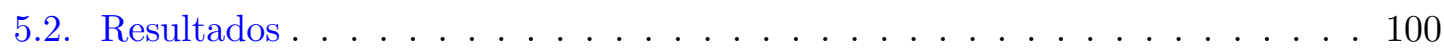

6. Conclusiones y recomendaciones 109

6.1. Conclusiones ....................... . . 109

6.2. Recomendaciones ..................... . . 111

$\begin{array}{ll}\text { A. Apéndice } & 113\end{array}$

A.1. Funciones especiales . . . . . . . . . . . . . . . . . . . 113

A.2. Cambios de variables útiles en el desarrollo de las DEA . . . . . . . . . . 115

A.3. Integrales útiles en el desarrollo de las DEA . . . . . . . . . . . . . . 117

A.4. Desarrollo de las DEA . . . . . . . . . . . . . . . . . 118

A.5. DEA entre superficies en presencia de gases claros . . . . . . . . . . . 136

A.6. DEA en presencia de gases opacos . . . . . . . . . . . . . . . . 138

A.7. Coeficiente de absorción medio . . . . . . . . . . . . . . . . . . 139 
$\begin{array}{ll}\text { Bibliografía } & 141\end{array}$

Nomenclatura 151

Acrónimos 


\section{Agradecimientos}

Este trabajo se realizó en el Instituto de Tecnología Cerámica. En el desarrollo del mismo, fue fundamental la participación de Ana Mezquita, Eva Vaquer y Salva Ferrer, quienes proporcionaron cuanta información se necesitó, participaron en incontables discusiones y efectuaron las medidas experimentales.

Los datos para validación experimental del modelo se obtuvieron en las instalaciones de La Platera y el Grupo Azulev. De estas experiencias debe destacarse la cooperación y la cordialidad de Manuel Gayet, Miguel Ángel Arnal y Antonio Alfaro.

Los directores de esta tesis también merecen una mención en estas líneas. Eliseo Monfort ha sido el principal impulsor de este trabajo y quien ha velado por que se lleve a cabo. Vicent Cantavella supervisó el trabajo teórico e hizo aportaciones esenciales como el algoritmo de integración basado en una cola de prioridades. 



\section{Resumen}

La etapa de cocción es, en muchos aspectos, la más importante del proceso de fabricación de baldosas cerámicas. En la cocción se producen cambios microestructurales en las baldosas que les proporcionan sus propiedades y aspecto finales. En el sector español, la cocción de baldosas cerámicas se realiza, desde los años 1990, casi exclusivamente en hornos monoestrato de rodillos, utilizando gas natural como combustible.

Se desarrolló un modelo matemático, basado en el método zonal, que permite simular la transferencia de energía en la cocción de baldosas cerámicas en hornos de rodillos. El método zonal requiere, en primer lugar, descomponer el horno en un conjunto de zonas, que pueden ser volúmenes o superficies, lo suficientemente pequeñas como para poder ser consideradas isotermas. La descomposición del horno de rodillos en zonas consistió en una división longitudinal en sectores, constituidos por zonas de diversos tipos: gases, baldosas, paredes y rodillos.

El método zonal permite calcular el intercambio de radiación térmica entre las zonas de manera rigurosa, lo que involucra el cálculo de tres conjuntos de áreas de intercambio, las cuales incorporan de manera gradual las propiedades radiantes de los elementos del sistema. Las áreas de intercambio directo son las más costosas desde el punto de vista computacional. Para conseguir que su cálculo fuera eficiente, se desarrollaron analíticamente las integrales múltiples que definen a estas áreas de intercambio, de manera que todos los posibles intercambios pudieron expresarse en términos de integrales simples o dobles. Además, se propuso un algoritmo de integración adaptativo, basado en una cola de prioridades, que permite evaluar las integrales resultantes de manera eficiente.

Tras plantear el intercambio radiativo entre las zonas, pudieron formularse balances de energía para cada una de ellas, incorporando otros flujos de energía, tales como los flujos de entalpía o el intercambio convectivo. Son muchos los términos que deben contemplarse en los balances de energía. Por ejemplo, para las baldosas, debe tenerse en cuenta el transporte de energía a su través por conducción, las reacciones químicas endotérmicas que éstas experimentan e incluso la liberación de especies gaseosas a los gases.

Los balances de energía planteados para cada zona constituyen, colectivamente, un complicado sistema de ecuaciones no lineales. Se desarrollaron dos algoritmos que permiten resolver dicho sistema. El primer algoritmo permite calcular la temperatura de todas las zonas, partiendo de las dimensiones del horno, de las propiedades de los materiales y de los flujos de materia entrantes (incluyendo los caudales de combustible y comburente). En el segundo algoritmo, se especifican temperaturas de consigna para los gases en lugar de los caudales de combustible en cada quemador, siendo estos últimos un resultado del cálculo.

Para validar el modelo, se llevaron a cabo una serie de determinaciones experimentales en tres hornos industriales, bajo condiciones normales de operación. Se recabaron todos los datos de entrada necesarios para poder efectuar el cálculo de las temperaturas de las 
zonas, las cuales se compararon con valores experimentales. Las máximas diferencias entre las temperaturas experimentales y las teóricas fueron inferiores al $10 \%$.

Una vez comprobada la validez del modelo, se realizaron una serie de simulaciones orientadas principalmente a estimar el efecto de modificar ciertas variables de proceso sobre el consumo energético del horno. Las variables estudiadas fueron: la temperatura del aire comburente, el espesor de las baldosas, la duración del ciclo de cocción, la temperatura máxima de cocción y el contenido en carbonatos en la composición. En todos los casos, se estudió la interacción de la modificación de dichas variables con el exceso de aire, debido a que industrialmente el caudal de aire comburente suele permanecer fijo.

Las simulaciones permitieron valorar el peso relativo de las variables estudiadas sobre el consumo energético del horno. Considerando unos intervalos de variación realizables industrialmente, el exceso de aire y el espesor de las baldosas resultaron ser las que más influencia tienen sobre el consumo de energía por unidad de producto. 


\section{Resum}

L'etapa de cocció és, en molts aspectes, la més important del procés de fabricació de taulells ceràmics. En la cocció es produeixen canvis microestructurals en els taulells que els proporcionen les seues propietats i aspecte finals. En el sector espanyol, la cocció de taulells ceràmics es realitza, des dels anys 1990, quasi exclusivament en forns monoestrat de corrons, amb la utilització de gas natural com a combustible.

Es va desenvolupar un model matemàtic, basat en el mètode zonal, que permet simular la transferència d'energia en la cocció de taulells ceràmics en forns de corrons. El mètode zonal requereix, en primer lloc, descompondre el forn en un conjunt de zones, que poden ser volums o superfícies, prou xicotetes com per a poder ser considerades isotermes. La descomposició del forn de corrons en zones va consistir en una divisió longitudinal en sectors, constituïts per zones de diversos tipus: gasos, taulells, parets i corrons.

El mètode zonal permet calcular l'intercanvi de radiació tèrmica entre les zones de manera rigorosa, la qual cosa involucra el càlcul de tres conjunts d'àrees d'intercanvi, les quals incorporen de manera gradual les propietats radiants dels elements del sistema. Les àrees d'intercanvi directe són les més costoses des del punt de vista computacional. Per a aconseguir que el seu càlcul fora eficient, es van desenvolupar analíticament les integrals múltiples que defineixen aquestes àrees d'intercanvi, de manera que tots els possibles intercanvis van poder expressar-se en termes d'integrals simples o dobles. A més, es va proposar un algoritme d'integració adaptatiu, basat en una cua de prioritats, que permet avaluar les integrals resultants de manera eficient.

Després de plantejar l'intercanvi radiatiu entre les zones, van poder formular-se balanços d'energia per a cadascuna d'aquestes, amb la incorporació d'altres fluxos d'energia, com ara els fluxos d'entalpia o l'intercanvi convectiu. Són molts els termes que han de contemplarse en els balanços d'energia. Per exemple, per als taulells, ha de tenir-se en compte el transport d'energia al seu través per conducció, les reaccions químiques endotèrmiques que aquestes experimenten i fins i tot l'alliberament d'espècies gasoses als gasos.

Els balanços d'energia plantejats per a cada zona constitueixen, collectivament, un complicat sistema d'equacions no lineals. Es van desenvolupar dos algoritmes que permeten resoldre aquest sistema. El primer algoritme permet calcular la temperatura de totes les zones, partint de les dimensions del forn, de les propietats dels materials i dels fluxos de matèria entrants (incloent els cabals de combustible i comburent). En el segon algoritme, s'especifiquen temperatures de consigna per als gasos en compte dels cabals de combustible en cada cremador, sent aquests últims un resultat del càlcul.

Per a validar el model, es van dur a terme una sèrie de determinacions experimentals en tres forns industrials, en condicions normals d'operació. Es van reunir totes les dades d'entrada necessàries per a poder efectuar el càlcul de les temperatures de les zones, les quals es van comparar amb valors experimentals. Les màximes diferències entre les temperatures experimentals i les teòriques van ser inferiors al $10 \%$. 
Una vegada comprovada la validesa del model, es van realitzar una sèrie de simulacions orientades principalment a estimar l'efecte de modificar algunes variables de procés sobre el consum energètic del forn. Les variables estudiades van ser: la temperatura de l'aire comburent, la grossària dels taulells, la durada del cicle de cocció, la temperatura màxima de cocció i el contingut en carbonats en la composició. En tots els casos, es va estudiar la interacció de la modificació d'aquestes variables amb l'excés d'aire, pel fet que industrialment el cabal d'aire comburent sol romandre fix.

Les simulacions van permetre valorar el pes relatiu de les variables estudiades sobre el consum energètic del forn. Atesos uns intervals de variació realitzables industrialment, l'excés d'aire i la grossària dels taulells van resultar ser les que més influència tenen sobre el consum d'energia per unitat de producte. 


\section{Abstract}

The firing stage is, in many ways, the most important ceramic tile manufacturing process stage. Firing gives rise to microstructural changes in the tiles, which give tiles their final properties and appearance. In the Spanish ceramic tile sector, tiles have been fired almost exclusively in single-deck roller kilns since the 1990s, using natural gas as fuel.

A mathematical model was developed, based on the zone method, which allows energy transfer in ceramic tile firing in roller kilns to be simulated. The zone method requires, first, breakdown of the kiln into a set of zones, which can be volumes or surfaces, sufficiently small to be deemed isothermal. The roller kiln zonal breakdown consisted of a longitudinal division into sectors, made up of various types of zones: gases, ceramic tiles, walls, and rollers.

The zone method enables the thermal radiation exchange between the zones to be rigorously calculated, involving calculation of three sets of exchange areas, which gradually incorporate the radiant properties of the system elements. The direct exchange areas are the most expensive from a computational viewpoint. To calculate these efficiently, the multiple integrals defining these exchange areas were analytically developed, so that all possible exchanges could be expressed in terms of single or double integrals. In addition, an adaptive integration algorithm, based on a priority queue, was developed for the resulting integrals to be efficiently evaluated.

After addressing the radiative exchanges between the zones, energy balances could be formulated for each zone, incorporating other energy flows, such as enthalpy flows and convective exchange. Many terms need to be considered in energy balances. For example, energy balances on ceramic tiles need to consider the conductive transport of energy through the tiles, the endothermic chemical reactions that the tiles undergo, and even the release of gaseous species into the gases.

The energy balances drawn up for each zone constitute, collectively, a complicated system of non-linear equations. Two algorithms were developed to solve this system. The first algorithm allowed the temperature of every zone to be calculated, based on the kiln dimensions, properties of the materials, and entering mass flows (including the fuel and combustion air flow rates). In the second algorithm, the temperature settings for the gases were specified, instead of the fuel flow rates at each burner, the fuel flow rates being obtained from the calculation.

To validate the model, a series of experimental determinations were conducted in three industrial kilns under typical operating conditions. All the input data required to calculate the zone temperatures were obtained, the calculated values then being compared with the experimental data. The maximum differences between the experimental temperature data and the theoretical values were lower than $10 \%$.

Once the validity of the model had been verified, a series of simulations were performed, aimed mainly at estimating how the modification of certain process variables affected kiln 
energy consumption. The following variables were studied: combustion air temperature, tile thickness, firing cycle time, peak firing temperature, and carbonate content in the tile composition. In every case, the study examined the interaction of the modification of these variables with excess air, as the combustion air flow rate is usually fixed in industrial practice.

The simulations allowed the relative weight of the variables on kiln energy consumption to be evaluated. On considering industrially practicable ranges of variation, excess air and tile thickness were found to be the variables that most influenced energy consumption per unit product. 
Deinde ponendo quod excessus calorum ferri supra calorem atmosphæræ thermometro inventum essent in progressione geometrica ubi tempora sunt in progressione arithmetica.

- Isaac Newton [1]

\section{Introducción}

\subsection{Tipos de baldosas cerámicas y proceso de fabricación}

Las baldosas cerámicas son materiales de construcción destinados al recubrimiento de suelos (pavimentos) y paredes (revestimientos). Se componen de un cuerpo cerámico o soporte de porosidad variable y, en la mayoría de los casos, de una capa superficial, de naturaleza mayoritariamente vítrea, denominada vidriado.

En la actualidad, la producción de pavimentos y revestimientos cerámicos se caracteriza por una amplia diversidad, tanto en sus formas, como en sus características técnicas y estéticas. Las baldosas cerámicas pueden clasificarse en función de su acabado superficial, uso al que van destinadas, características del proceso de fabricación y propiedades del producto acabado. Las normas UNE-EN 14411 [2] e ISO 13006 [3] proponen una clasificación basada, por una parte, en el método de conformado utilizado y, por otra, en la porosidad de las baldosas según un ensayo normalizado (absorción de agua).

Resulta más práctico, al menos para los fines de la presente memoria, utilizar una clasificación alternativa aunque, en cierta medida, equivalente a la normalizada. En concreto, se utilizará un subconjunto de las categorías propuestas en la «Guía de la baldosa cerámica» $[4]$ :

Azulejo. Se trata de baldosas con elevada porosidad abierta (superior al $10 \%$ en términos de absorción de agua) y elevada estabilidad dimensional como consecuencia de la formación de fases cálcicas en el soporte durante la cocción. La porosidad reduce su peso y favorece su adherencia a las paredes, pero disminuye su resistencia mecánica. Estas características hacen que se empleen esencialmente para el revestimiento de paredes.

Gres (esmaltado). Los soportes del gres tienen una porosidad baja, por lo que sus características mecánicas son más adecuadas para su uso como pavimento. Aunque tradicionalmente así ha sido, no es extraño que se empleen también como revestimientos. Al igual que los azulejos, las baldosas de gres siempre cuentan con un vidriado superficial obtenido por aplicación de un esmalte sobre su cara vista y, por ello, es innecesario el uso del calificativo «esmaltado».

Gres porcelánico. Se caracteriza por presentar una porosidad abierta extremadamente baja (absorción de agua inferior al 0,5 \%). La porosidad reducida les proporciona unas propiedades mecánicas superiores, así como una mayor resistencia a la helada, lo que las hace aptas para exteriores en zonas frías. En ocasiones, en lugar de obtener el aspecto estético de este producto por esmaltado, se decora el propio soporte. Aún así, incluso estos productos casi siempre cuentan con una capa de vidriado transparente que les confiere una mayor dureza, aunque por motivos tradicionales se las considere baldosas «no esmaltadas». 
Es también conveniente distinguir si el soporte de las baldosas se ha producido a partir de materias primas de coloración roja o blanca (tras la cocción). En el sector español de fabricación de baldosas, ubicado principalmente en la provincia de Castellón, se producen ambos tipos de productos. La producción de azulejos con soporte blanco tras la cocción, aunque significativa, es menor que la producción de azulejos rojos. Del mismo modo, la producción de gres rojo predomina frente a la de gres blanco. Sin embargo, aunque puede que sea técnicamente posible obtener baldosas de gres porcelánico con materias primas de cocción roja [5], la producción de las mismas en el sector español es escasa. El grueso de la producción de gres porcelánico se obtiene a partir de materias primas de cocción blanca.

El proceso de fabricación de baldosas cerámicas se desarrolla en varias fases sucesivas. La secuencia más extendida en la actualidad en el sector español involucra la molturación de las materias primas por vía húmeda, el conformado de las piezas por prensado uniaxial y la cocción simultánea del soporte y el esmalte (monococción). En la figura 1.1 se muestran las principales etapas que se dan en esta modalidad de fabricación [6].

El proceso comienza con la selección de las materias primas que deben formar parte de la composición de partida. Las materias primas utilizadas son fundamentalmente: arcillas (que pueden ser de coloración roja o blanca), caolines, feldespatos, cuarzo, carbonatos y otras de carácter minoritario que dependen del tipo de producto fabricado. Las materias primas se suelen utilizar, por lo general, tal y como se extraen de la mina o cantera, o después de someterlas a un mínimo tratamiento.

El origen natural de las materias primas exige, en la mayoría de los casos, una homogeneización previa que asegure la continuidad de sus características. Por ello, una vez llegan al centro de producción, éstas son depositadas en cúmulos o montones, que pueden estar a cielo abierto o total o parcialmente cubiertos, donde se homogeneizan y se secan parcialmente. Esta homogeneización puede ir precedida o seguida de un proceso de trituración o molturación primaria.

Tras la homogeneización, las materias primas que constituyen la composición cerámica se someten a un proceso de molturación, el cual se realiza habitualmente por vía húmeda en molinos de bolas. A la suspensión resultante (barbotina), se le elimina, mediante secado por atomización, parte del agua que contiene, hasta alcanzar el contenido en humedad requerido.

En el secado por atomización, la barbotina pulverizada en finas gotas, entra en contacto con una corriente de aire caliente para obtener un producto sólido granular de bajo contenido en agua. El tiempo de contacto entre la suspensión pulverizada y el gas caliente (proveniente de un quemador convencional o de los gases de escape de una turbina de cogeneración) es pequeño, por lo que se produce una evaporación violenta del agua que contiene cada gota, de manera que el granulo atomizado resultante retiene parte de la esfericidad de las gotas.

Una vez finalizado el proceso de preparación de las materias primas, se procede al conformado de las piezas. Este proceso se realiza habitualmente por prensado uniaxial en semiseco (5-8\% de humedad en base seca), mediante el uso de prensas hidráulicas. En algunos casos se moldea por extrusión para conferir al producto final un aspecto estético determinado: por ejemplo, el pavimento rústico.

Después del proceso de conformado, es necesario secar las piezas. De esta forma se consigue reducir su contenido en humedad lo suficiente para poder llevar a cabo las operaciones 


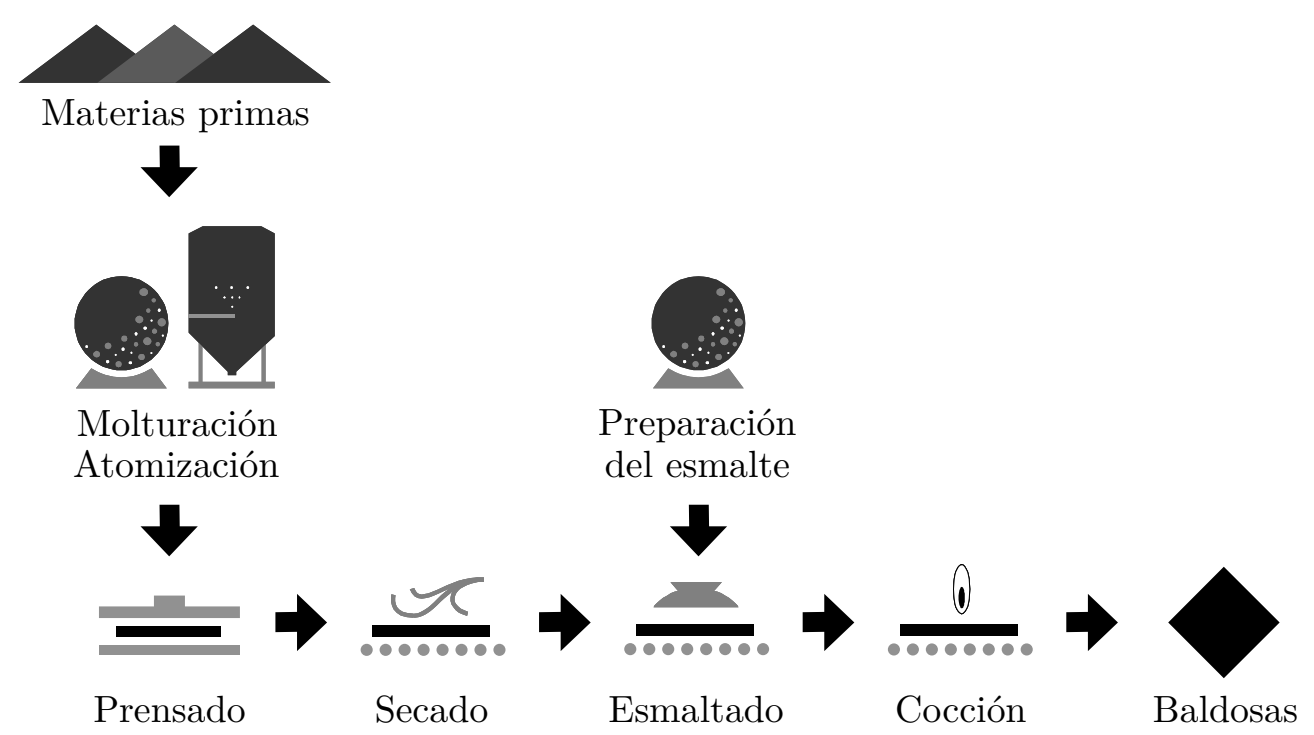

Figura 1.1. Proceso de fabricación de baldosas cerámicas por monococción.

posteriores de esmaltado y cocción. También puede haber un secado adicional tras la etapa de esmaltado. El secado de las piezas se consigue poniéndolas en contacto, en el interior de un secadero, con gases calientes aportados por un quemador o procedentes de la chimenea de enfriamiento del horno.

El esmaltado consiste en la aplicación por distintos métodos de una o varias capas de vidriado, que cubre la superficie de la pieza. Este tratamiento se realiza para conferir al producto cocido una serie de propiedades técnicas y estéticas, como impermeabilidad, facilidad de limpieza, brillo, color, textura y resistencia química y mecánica. El esmaltado de las baldosas se realiza en continuo. Los esmaltes se aplican en cortina, por pulverización o en seco. También se aplican diversas decoraciones por serigrafía, huecograbado o impresión digital, dependiendo del acabado final que se quiera conseguir. Los esmaltes están compuestos por fritas y por material no fritado. El proceso de preparación de esmaltes consiste normalmente en someter a la frita y los otros componentes a una molienda, en molinos de bolas de alúmina, hasta obtener una suspensión acuosa cuyas características dependen del método de aplicación que se vaya a utilizar.

Tras la etapa de esmaltado se realiza la cocción del esmalte en los procesos de bicocción, o la cocción del esmalte y del soporte conjuntamente en los procesos de monococción. La cocción consiste en someter a las piezas a un ciclo térmico, durante el cual tiene lugar una serie de transformaciones en la pieza que provocan cambios en su microestructura y les confiere las propiedades finales deseadas. Esta etapa es una de las más importantes del proceso de fabricación, ya que de ella dependen gran parte de las características del producto final.

En ocasiones, se realizan tratamientos mecánicos (pulido, biselado, rectificado, etc.) sobre las baldosas una vez cocidas. Estos tratamientos se introdujeron inicialmente para los productos de gres porcelánico no esmaltado, pero progresivamente se han extendido a una parte de los productos esmaltados. 


\subsection{Consumo de energía en la fabricación de baldosas cerámicas}

La fabricación de productos cerámicos requiere un gran aporte de energía térmica para realizar los procesos de secado y cocción. En la actualidad, el combustible mayoritariamente empleado en la industria cerámica de Castellón es el gas natural. La energía eléctrica se consume en todas las etapas del proceso, en mayor medida en las fases de molienda de las materias primas y de conformado de las piezas, pero su consumo global es muy inferior al de energía térmica.

Según el estudio sectorial más reciente, realizado en el año 2008, el consumo anual de gas natural como demanda de energía final en el sector alcanzó los $50 \cdot 10^{9} \mathrm{MJ}$, calculados a partir del poder calorífico superior (PCS) del combustible. La energía eléctrica consumida por el sector proviene tanto de la red eléctrica como de los sistemas de cogeneración. El consumo total de electricidad ascendió, en 2008 , a $4,3 \cdot 10^{9} \mathrm{MJ}[7,8]$.

En la figura 1.2 se muestra la distribución media de los consumos de energía en una instalación de fabricación de baldosas cerámicas por monococción. En lo referente al consumo de energía térmica en el proceso de fabricación, éste se produce principalmente en tres etapas: el secado por atomización, el secado de los soportes recién conformados y la cocción de las baldosas. La distribución media del consumo de energía térmica en estas tres etapas también se indica en la figura 1.2.

El PCS del combustible incorpora el calor de condensación del vapor de agua generado en la combustión. La condensación de vapor de agua en el interior de los equipos empleados para el secado y cocción de productos cerámicos causaría serios problemas. No es realista considerar que la energía liberada en la condensación es aprovechable. Por este motivo, en lo sucesivo se expresarán los consumos energéticos en base al poder calorífico inferior (PCI), que excluye el calor de condensación del agua y, por consiguiente, cuantifica el calor útil aportado. Teniendo en cuenta esta consideración, según la bibliografía, el consumo medio de energía térmica en el sector español de fabricación de baldosas cerámicas es de $4,6 \mathrm{MJ} \mathrm{kg}^{-1}$ de producto cocido, siendo la etapa de cocción la de mayor consumo, con un valor medio de $2,6 \mathrm{MJ} \mathrm{kg}^{-1}$ [8]. En el sector italiano, donde se utiliza la misma tecnología, se tienen valores similares [9].

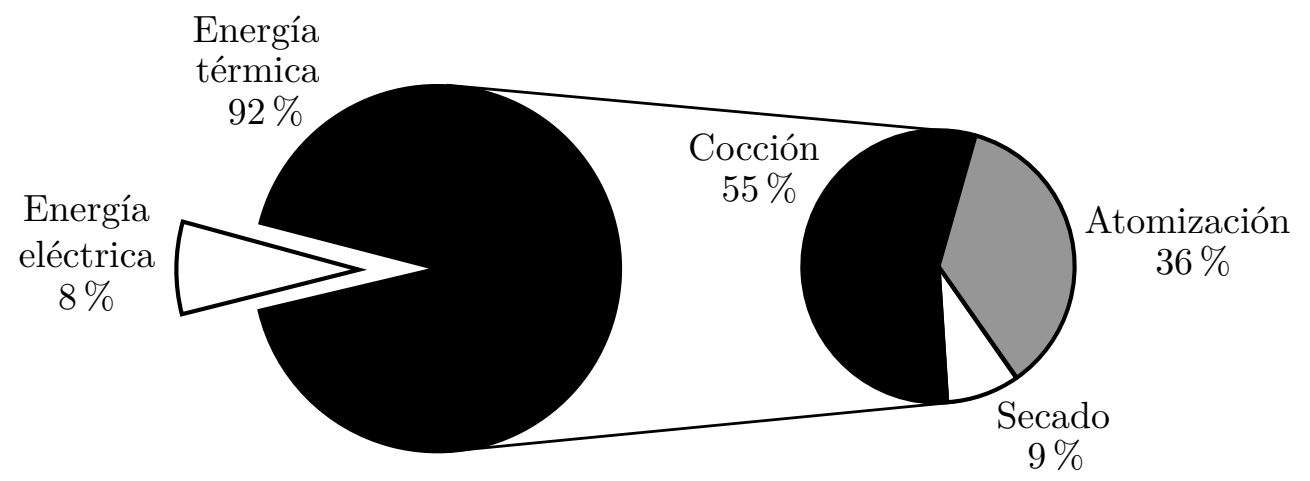

Figura 1.2. Distribución del consumo energético en la fabricación de baldosas cerámicas [7]. 

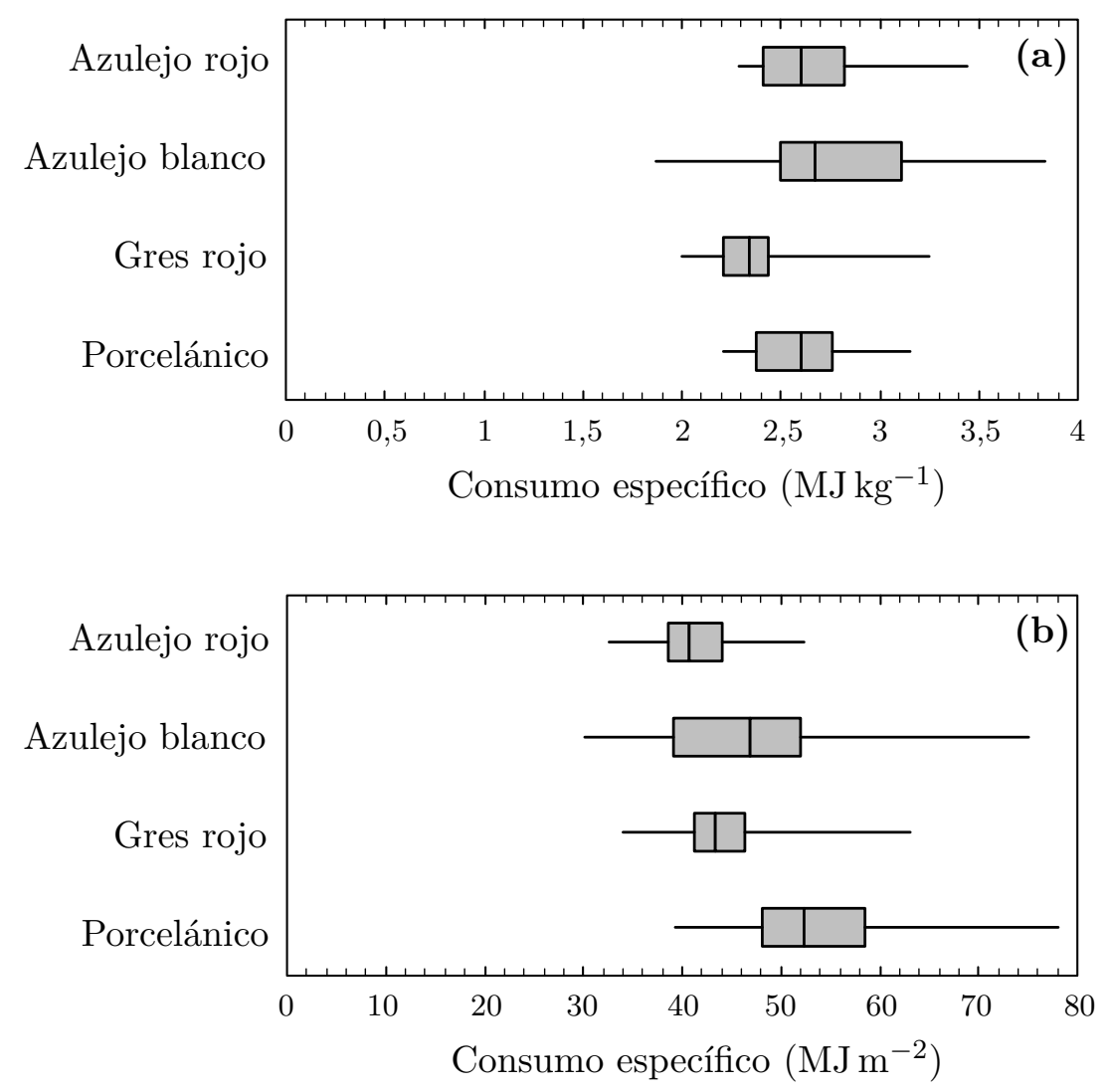

Figura 1.3. Cuartiles e intervalos de variación del consumo energético en la cocción de baldosas cerámicas según el tipo de producto [8].

Como se observa en la figura 1.3, el consumo energético en la cocción depende del tipo de producto [8]. Estas diferencias se justifican por una serie de factores, en los cuales se ahondará más adelante (capítulo 5). Los productos de gres rojo son los que requieren un menor aporte de energía por unidad de masa de producto cocido, en comparación con los azulejos y el gres porcelánico (figura 1.3(a)). Sin embargo, si se expresan los consumos por unidad funcional $\left(\mathrm{m}^{2}\right)$, el menor peso específico de los azulejos $\left(\mathrm{kg} \mathrm{m}^{-2}\right)$ compensa en buena medida su mayor consumo por unidad de masa. En estos términos, el gres porcelánico resulta ser el producto que involucra una mayor demanda energética (figura 1.3(b)) [7].

\subsection{Cocción de baldosas cerámicas}

La etapa de cocción consiste en someter a las piezas a un ciclo térmico controlado durante el que tienen lugar una serie de transformaciones físico-químicas permanentes e irreversibles en el material, que modifican su microestructura y le confieren las propiedades finales (tabla 1.1). En este sentido, la etapa de cocción es, en muchos aspectos, la más importante del proceso de fabricación, pues es en la que el producto final alcanza las características técnicas y estéticas especificadas: dimensiones, planaridad, ortogonalidad, tonalidad, resistencia mecánica, porosidad, acabado superficial, etc. 


\begin{tabular}{cc} 
Temperatura $\left({ }^{\circ} \mathrm{C}\right)$ & Transformaciones \\
\hline ambiente-150 & $\begin{array}{c}\text { Eliminación del agua libre } \\
\text { hidratos de hierro }\end{array}$ \\
\hline $350-650$ & Eliminación del agua de constitución \\
\hline $400-600$ & Combustión de sustancias orgánicas \\
\hline 573 & Transformación polimórfica cuarzo $\alpha \rightarrow$ cuarzo $\beta$ \\
\hline $700-800$ & Inicio de la fusión de los álcalis y óxidos de hierro \\
\hline $800-900$ & Descomposición de los carbonatos. Oxidación del carbón \\
\hline 1000 & Inicio de la fusión de composiciones con CaO y FeO con \\
formación de silicatos
\end{tabular}

Tabla 1.1. Principales transformaciones físico-químicas que tienen lugar durante el tratamiento térmico de las baldosas cerámicas.

Los fenómenos que se producen durante la cocción deben tener lugar de una manera gradual y controlada, de lo contrario pueden causar defectos permanentes en el producto [10]. Por ello, el proceso de cocción se lleva a cabo de acuerdo a un plan previamente establecido que se conoce con el nombre de curva de cocción o, más frecuentemente, ciclo de cocción, en el que se establece la variación de la temperatura con respecto al tiempo.

La forma de la curva de cocción depende fundamentalmente de la composición del soporte del producto que se fabrica (azulejos, gres o porcelánico). No obstante, cualquier curva de cocción incluye, de una manera más o menos definida, las etapas que se indican en la figura 1.4:

1. Etapa inicial de calentamiento. Un factor que limita la velocidad de calentamiento en esta etapa es la humedad residual de las piezas, que puede provocar la rotura de las mismas si la velocidad es excesiva.

2. Etapa de oxidación y descomposición. En esta etapa se produce la oxidación de compuestos de carbono y de las formas reducidas del óxido de hierro, así como la descomposición de los carbonatos de calcio y de magnesio (especialmente en composiciones de azulejo). Es necesario que todas estas reacciones se hayan completado antes de que se produzca la fusión del esmalte y, por este motivo, se reduce la velocidad de calentamiento.

3. Etapa final de calentamiento. Una vez se han completado las reacciones químicas en los intervalos de temperatura apropiados, se incrementa de nuevo la velocidad de calentamiento. 


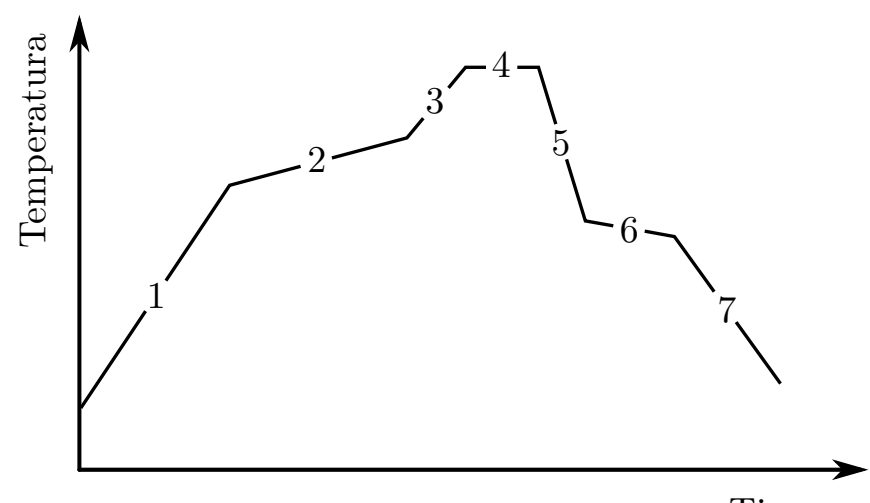

Tiempo

Figura 1.4. Curva de cocción típica.

4. Cocción propiamente dicha. Se lleva a cabo a una temperatura comprendida entre 1100 y $1200^{\circ} \mathrm{C}$, la cual varía principalmente en función del tipo de producto. Las especificaciones del producto se consiguen en gran medida en esta etapa.

5. Enfriamiento rápido. Debido a la elevada resistencia del material al choque térmico, este tramo transcurre a la mayor velocidad posible.

6. Enfriamiento lento. A una temperatura próxima a los $573^{\circ} \mathrm{C}$, la resistencia al choque térmico del material disminuye bruscamente como consecuencia de la transformación polimórfica del cuarzo $\beta$ en cuarzo $\alpha$. Esto limita la velocidad a la que puede llevarse a cabo el enfriamiento en torno a la temperatura señalada.

7. Enfriamiento final. Superado el punto crítico, las piezas se vuelven a enfriar lo más rápidamente posible.

\subsection{Hornos monoestrato de rodillos}

La cocción de baldosas cerámicas sufrió una profunda evolución técnica en los años 1980 y principios de los 1990 [11]. La tecnología utilizada anteriormente consistía en la bicocción tradicional, que se realizaba en hornos túnel de llama directa para la cocción del soporte y hornos de canales para la cocción del esmalte. La disponibilidad de combustibles gaseosos prácticamente exentos de azufre (propano y, más tarde, gas natural), que posibilitan poner en contacto directo los gases de combustión con el producto sin comprometer su calidad, promovió el desarrollo de composiciones y esmaltes para monococción en ciclos rápidos.

La cocción rápida se efectúa en hornos monoestrato de rodillos. En estos hornos, a diferencia de los hornos túnel tradicionales en los que las piezas se apilaban en vagonetas, las baldosas se alimentan formando una única capa. La capa de baldosas se sustenta sobre un banco de rodillos de unos $5 \mathrm{~cm}$ de diámetro cuya rotación realiza la movimentación de las mismas. Los rodillos atraviesan transversalmente la sección de paso de los gases del horno, dividiéndola en dos canales y consiguiendo de este modo una gran exposición de las piezas a los gases.

La implantación generalizada de los hornos monoestrato se justifica por una serie de ventajas frente a los tradicionales [12]: 
- Ciclos de cocción más cortos. La biccocción tradicional incluía una primera cocción del soporte de 30-40 h y una segunda cocción del soporte esmaltado de 10-15 h. La monococción en hornos de rodillos involucra la cocción simultánea del soporte y el esmalte en apenas 45-60 min.

- Control más ajustado de la operación. Debido a la mayor uniformidad de temperatura en la sección transversal del horno, puede conseguirse un mejor control de la curva de cocción.

- Mayor eficiencia energética que deriva en buena medida de una mayor razón carga útil/carga total que en los hornos tradicionales.

- Menor inercia térmica como consecuencia del aislamiento ligero del horno y del bajo peso del material contenido en el horno. Esta característica dota a estos hornos de gran flexibilidad que permite interrumpir y reemprender la producción sin notables dificultades, así como modificar rápidamente la curva de cocción.

Las anchuras típicas de los hornos, junto con las temperaturas máximas de cocción utilizadas, hacen que el uso de rodillos metálicos sea prácticamente inviable debido a la deformación (combadura) que éstos experimentan [13,14]. En su lugar se emplean rodillos cerámicos, los cuales soportan mejor las altas temperaturas, aunque también exhiben una menor resistencia al choque térmico, lo que es particularmente relevante en su limpieza y sustitución.

La utilización de un sistema de movimentación de las piezas basado en rodillos sin soporte refractario ciertamente repercute en muchas de las ventajas enumeradas anteriormente. Sin embargo, los rodillos interfieren en la transmisión de calor por la parte inferior de la pieza e influyen en la planaridad de las baldosas [15].

En la figura 1.5 se muestra una vista general de un horno monoestrato de rodillos para la cocción de baldosas cerámicas. Los hornos monoestrato de rodillos están basados en una estructura modular portante, ejecutada en carpintería metálica, que sustenta la mampostería aislante. Los módulos tienen unos $2 \mathrm{~m}$ de longitud y una anchura útil para el paso de piezas que varía en el intervalo 1,5-3 m. Los hornos de menor producción constan de alrededor de 30 módulos, pero es frecuente que tengan 50 o más.

La ejecución de los diversos módulos del horno difiere según la parte del horno de la que forman parte. Los hornos de rodillos pueden dividirse en dos partes bien diferenciadas: el calentamiento y el enfriamiento, atendiendo a si en ellas se incrementa o se disminuye la temperatura de las piezas. A continuación se describen brevemente cada una de ellas $\mathrm{y}$, seguidamente, se exponen algunos detalles relativos al funcionamiento del horno en su conjunto.

\subsubsection{Calentamiento}

La zona de calentamiento del horno comprende entre el 55 y el $60 \%$ de la longitud total del horno. En ella se incrementa la temperatura de las piezas hasta la temperatura máxima de cocción, que se mantiene durante el tiempo especificado. En la figura 1.6 se muestra un esquema de esta parte del horno. 


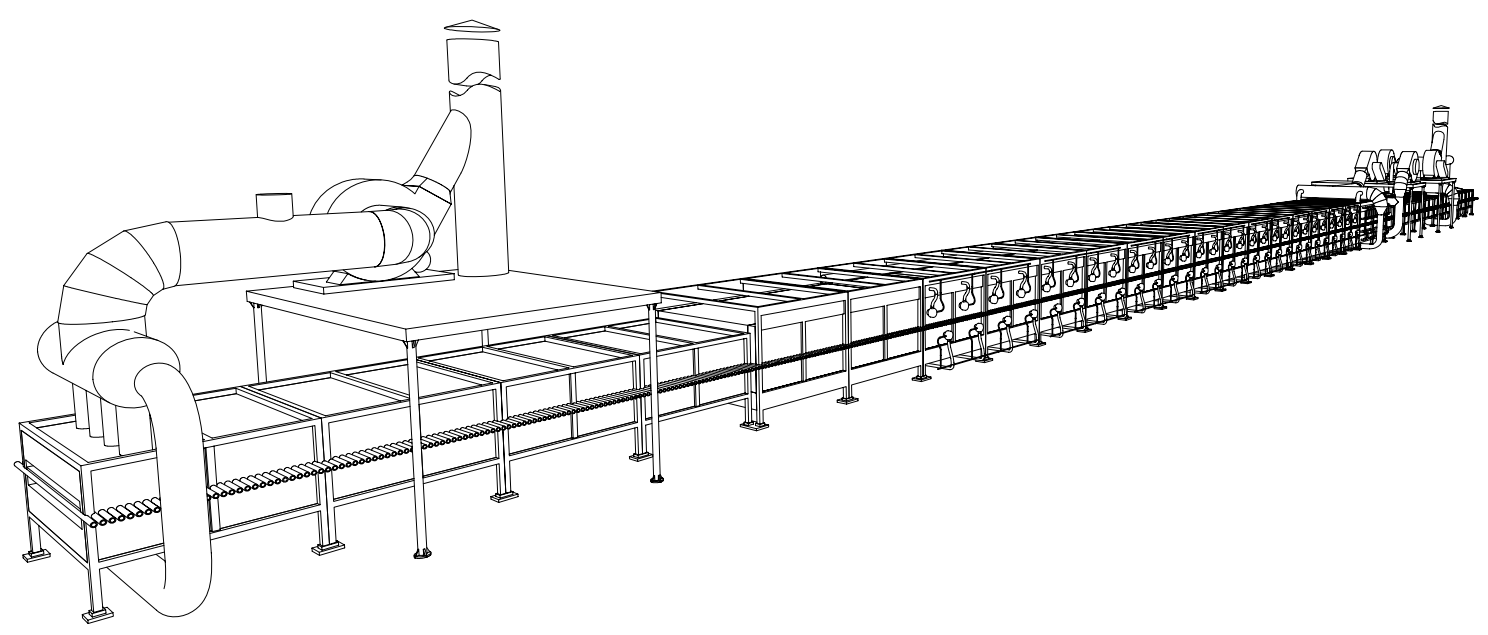

Figura 1.5. Horno monoestrado de rodillos.

\subsubsection{Aspiración de humos}

En esta zona se localiza el acceso de las piezas al horno y es donde se produce la aspiración de los humos para ser evacuados por la chimenea destinada a tal efecto. Esta parte del horno no está dotada de un sistema de aporte de calor autónomo. El calentamiento de las piezas se produce al circular los humos en contracorriente al sentido de avance de las mismas.

Las tomas de aspiración de humos están distribuidas en diversos puntos de extracción por encima y por debajo del banco de rodillos, dotados con válvulas de mariposa para regular el tiro. Estas tomas se localizan generalmente en la entrada del horno, aunque, en ocasiones, la aspiración de humos está dividida en los dos primeros módulos. De este modo, se consigue actuar en cierta medida sobre la velocidad de calentamiento inicial de las piezas.

El motoventilador de evacuación de humos no suele estar diseñado para operar a temperaturas elevadas [14]. Habitualmente la temperatura de trabajo pertenece al intervalo 120-250 ${ }^{\circ} \mathrm{C}$. Para alcanzar estas temperaturas, se dispone de un sistema de dilución que introduce aire ambiente en la corriente de humos, justo aguas arriba del ventilador. Este aire de dilución, junto con el aire parásito que accede por la boca del horno, puede llegar a constituir más de $\frac{2}{3}$ del caudal de gases en la chimenea de humos [16], de donde se deduce que la temperatura de los humos en la entrada del horno es de al menos 300 o $400{ }^{\circ} \mathrm{C}$.

Debido a las relativamente bajas temperaturas que se dan en esta parte del horno (inferiores a $800^{\circ} \mathrm{C}$ ), el espesor del aislamiento es comparativamente reducido. El aislamiento de las paredes y la bóveda es de fibra aislante, en placas rígidas ancladas en la estructura metálica de los módulos. Para la solera se emplea albañilería aislante de mayor resistencia mecánica, de modo que no se deteriore en caso de que caigan piezas rotas y en la eliminación de las mismas. 


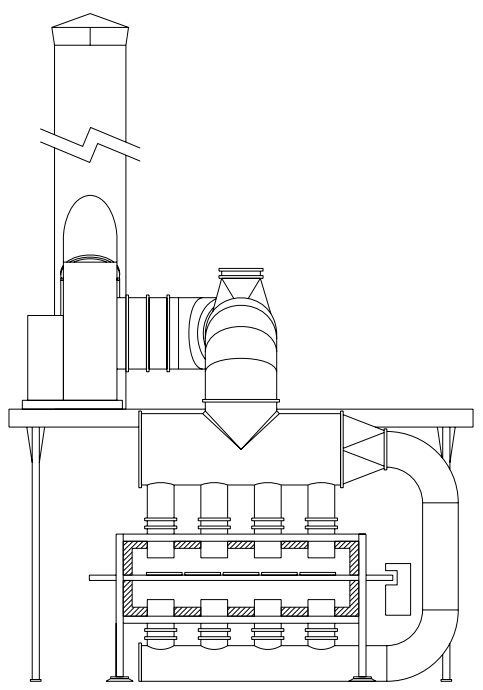

Aspiración de humos

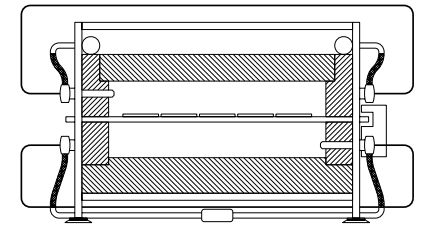

Cocción

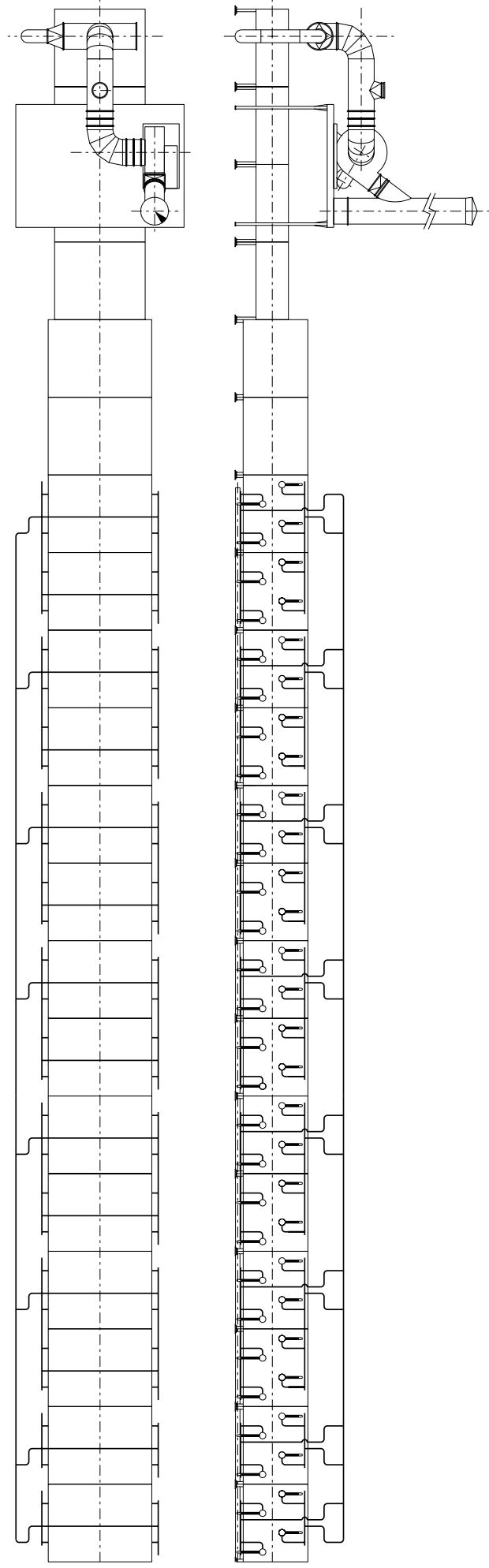

Figura 1.6. Esquema de la zona de calentamiento. 


\subsubsection{Precalentamiento y cocción}

Se suele distinguir entre la zona denominada (impropiamente) precalentamiento y la zona de cocción, siendo esta última donde se mantiene la temperatura máxima de cocción. No obstante, salvo quizás por una mejor calidad del aislamiento en la zona de cocción, no existen grandes diferencias entre ellas en términos constructivos y, por este motivo, se describirán conjuntamente.

Es en estas partes del horno donde se localiza el principal sistema de aporte de calor al horno: los quemadores. Éstos están dispuestos en las paredes laterales por encima y por debajo del banco de rodillos. En los quemadores que se utilizan habitualmente, el combustible y el aire comburente se mezclan directamente en la tobera de salida, que eyecta los productos de la combustión al interior del horno. La turbulencia generada favorece la uniformidad en la sección transversal del horno. La existencia de gradientes transversales de temperatura puede causar defectos o falta de consistencia en la producción, ya que las piezas podrían quedar sometidas a ciclos de cocción que se aparten sensiblemente del nominal, dependiendo de su posición a lo ancho del horno [17].

Cada módulo dotado de quemadores dispone de cuatro unidades sobre y bajo el banco de rodillos. El número total de quemadores está pues condicionado por la longitud del horno y habitualmente supera los 150. La disposición es a tresbolillo, alternándose las paredes en las que se encuentran instalados los sucesivos quemadores a lo largo del horno. Asimismo, la disposición en el canal inferior está invertida con respecto a la del canal superior, de manera que los quemadores superiores e inferiores se encuentran en posiciones antisimétricas (figura 1.6). Sin embargo, no es extraño que determinadas zonas solamente cuenten con quemadores en alguno de los canales o que haya quemadores apagados suministrando aire frío. En segunda y «tercera» cocción, por ejemplo, sólo suelen instalarse quemadores por encima del banco de rodillos.

Los quemadores están agrupados formando unos conjuntos que se denominan anillos. Los anillos suministran una presión de gas natural equitativa a cada quemador del grupo. El control de temperatura actúa sobre una válvula motorizada que regula cada anillo en su conjunto. En el precalentamiento, los anillos suelen comprender los quemadores de las partes superior o inferior de dos módulos. En la zona de máxima temperatura, donde se requiere un control de temperatura más estricto, los anillos agrupan los quemadores de un único módulo.

Los módulos en esta zona presentan una mayor sección transversal pues tienen que albergar un aislamiento de mayor espesor. La parte de las paredes expuesta a los humos está construida con ladrillos refractarios, revestidos con fibras refractarias y rematados con una placa aislante de cobertura en la parte exterior. La bóveda se realiza usualmente con bloques de refractario ligero, suspendidos con anclajes metálicos que se apoyan en la estructura de los módulos. La solera está totalmente construida en albañilería aislante.

El final de la zona de cocción está delimitado por una barrera física, constituida por un muro transversal que secciona casi totalmente el canal, tanto en la parte superior como en la inferior. De este modo se limita la influencia entre las zonas de enfriamiento y cocción. Dispositivos similares, denominados cortafuegos, pueden situarse en lugares estratégicos a lo largo de todo el calentamiento. Los cortafuegos obstaculizan la transferencia de radiación térmica entre las regiones que delimitan. 


\subsubsection{Enfriamiento}

La figura 1.7 muestra esquemáticamente la parte del horno en la que se reduce la temperatura de las baldosas hasta valores próximos a la temperatura ambiente. A diferencia de lo que sucede en la región de calentamiento del horno, hay una conexión más directa entre las zonas en las que se divide el enfriamiento y las etapas indicadas en $§ 1.3$.

\subsubsection{Enfriamiento rápido}

El sistema de enfriamiento rápido consiste esencialmente en tubos de soplado dispuestos por encima y por debajo del banco de rodillos. Se trata de tubos transversales a los que se les han practicado una serie de perforaciones a través de las cuales se proyecta aire sobre las baldosas. El objetivo es disminuir la temperatura de las piezas desde la temperatura de cocción hasta valores próximos, pero superiores, a la temperatura de transformación del cuarzo $\left(573^{\circ} \mathrm{C}\right)$. Destaca la rapidez con la que se desarrolla esta etapa, dada la reducción de temperatura que involucra. Para hornos cortos (tales como el mostrado en la figura 1.7) esta zona apenas comprende dos módulos, lo que pone de manifiesto los grandes volúmenes de aire involucrados.

Esta zona se sitúa a continuación de la zona de cocción, por lo que se construye con materiales adecuados para soportar altas temperaturas (normalmente idénticos a los empleados en la zona de cocción).

\subsubsection{Enfriamiento indirecto}

Esta parte del horno está designada a que se produzca la transformación polimórfica del cuarzo de manera controlada. Esta transformación comporta una contracción brusca de las piezas, que debe producirse con lentitud y con la mayor simultaneidad posible en todo su seno para minimizar las tensiones derivadas del proceso. A tal efecto, en esta zona, el dispositivo para la disipación de calor consiste en una serie de tubos intercambiadores de calor por los que circula aire aspirado del ambiente. La denominación de esta zona se debe a que no existe contacto directo entre el aire que circula por los tubos y la atmósfera del horno. El sentido de circulación del aire por los tubos se alterna en tubos sucesivos de manera que no se establezca un sentido preferencial de enfriamiento.

La sección de esta zona es de nuevo más reducida pues un aislamiento ligero es suficiente para las temperaturas que se dan desde esta zona hasta el final del horno. La zona está delimitada por sendas barreras transversales separadoras.

\subsubsection{Enfriamiento final}

Una vez completada la transformación del cuarzo, se reemprende el enfriamiento «directo» de las piezas de modo que su temperatura se reduzca hasta unos $100^{\circ} \mathrm{C}$. De nuevo, se emplean tubos transversales perforados para proyectar aire sobre las piezas, por encima y por debajo del banco de rodillos. En la bóveda se encuentran instaladas una serie de tolvas colectoras que captan el aire utilizado en el enfriamiento para descargarlo en un colector general. El aire extraído se vierte a la atmósfera a través de una chimenea dotada de un 


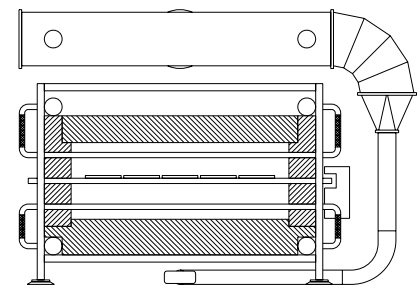

Enfriamiento rápido

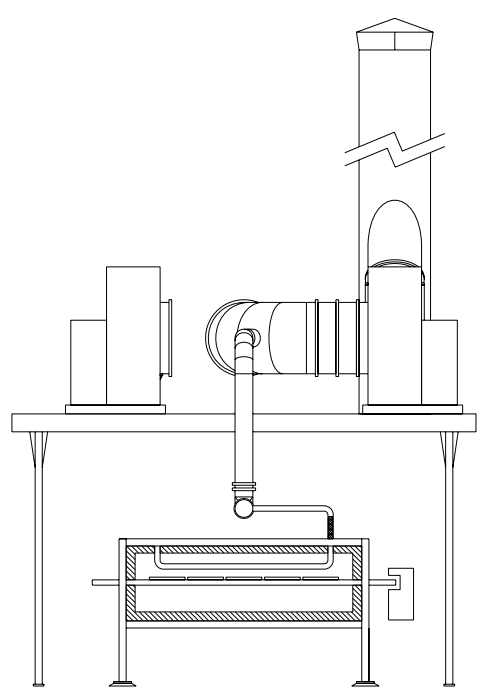

Enfriamiento indirecto
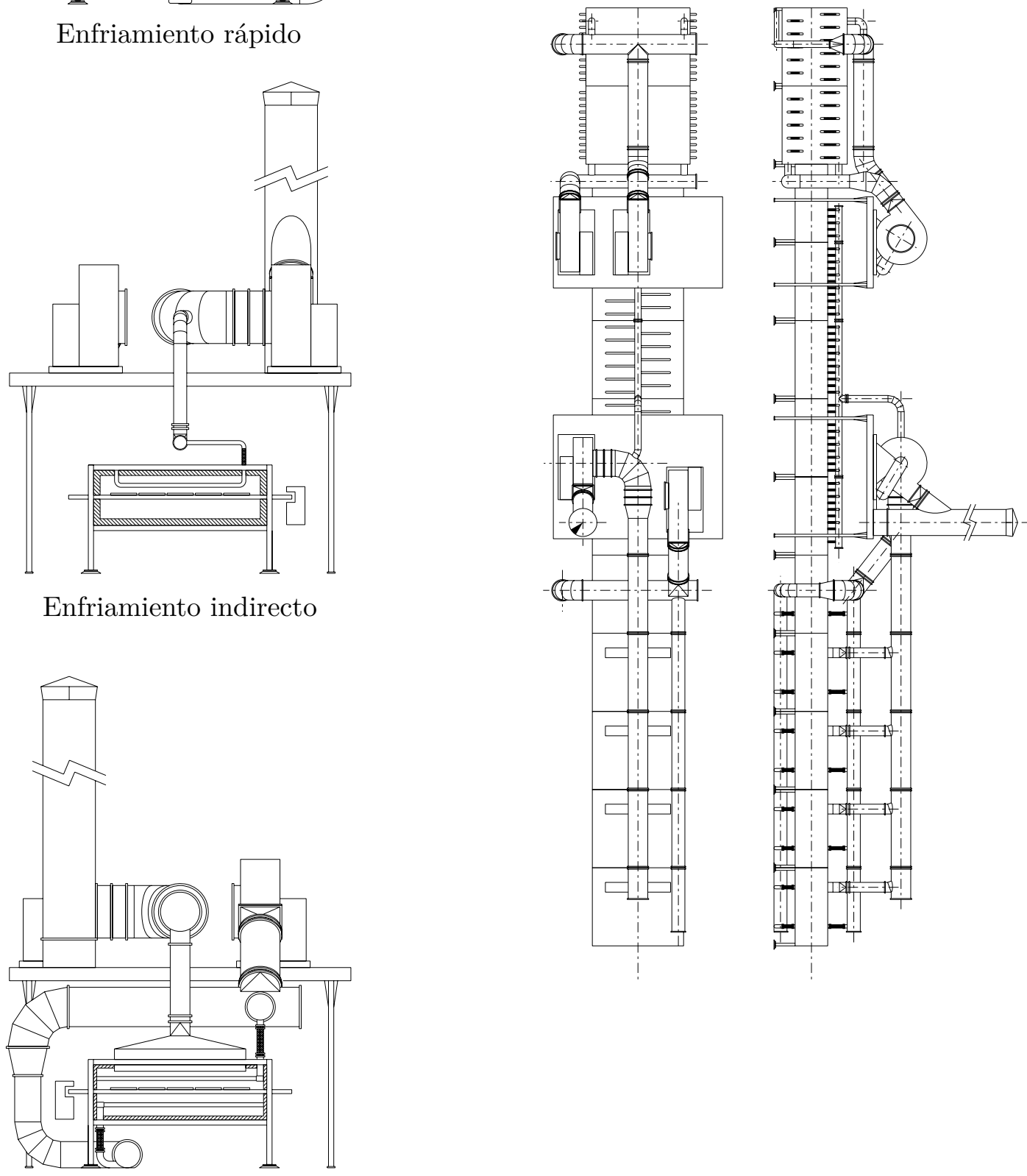

Enfriamiento final

Figura 1.7. Esquema de la zona de enfriamiento. 
ventilador que, con frecuencia, es también el responsable del trasiego de aire por el sistema de enfriamiento indirecto.

\subsubsection{Operación del horno}

\subsubsection{Circulación de gases}

En la figura 1.8 se indican las diversas entradas y salidas de gases en el horno. El ventilador AC impulsa el aire comburente hasta los quemadores. Los productos de la combustión se incorporan a la corriente gaseosa inducida por la depresión generada por el ventilador de la chimenea de humos $\mathrm{CH}$. Puesto que las piezas acceden al horno por la zona de aspiración de humos, en el calentamiento los gases circulan en contracorriente al sentido de avance de las piezas. Los ventiladores ER y EF se encargan del aporte de aire de enfriamiento rápido y final, respectivamente. En la figura 1.8, el ventilador CE es responsable de la evacuación del aire de enfriamiento y de la circulación de aire por el sistema de enfriamiento indirecto, aunque en ocasiones estas corrientes pueden estar separadas, como se apunta más adelante.

La distribución de presiones estáticas en el interior del horno determina la circulación general de gases y, por tanto, es de fundamental importancia en su correcta operación [18]. Los ventiladores de impulsión generan una presión positiva en el interior del horno con respecto a la presión atmosférica. Los ventiladores de extracción, por el contrario, provocan depresiones. Por tanto, en la entrada del horno, donde se produce la aspiración de humos, el horno se encuentra en depresión. Desde la entrada del horno hacia la zona de máxima temperatura, la presión aumenta hasta alcanzar un valor máximo, habitualmente positivo. Tras este máximo, la presión disminuye de nuevo gradualmente en la zona de enfriamiento pero, debido a los múltiples puntos de inyección y extracción de aire, no es posible ofrecer un perfil típico de presiones estáticas en el enfriamiento final.
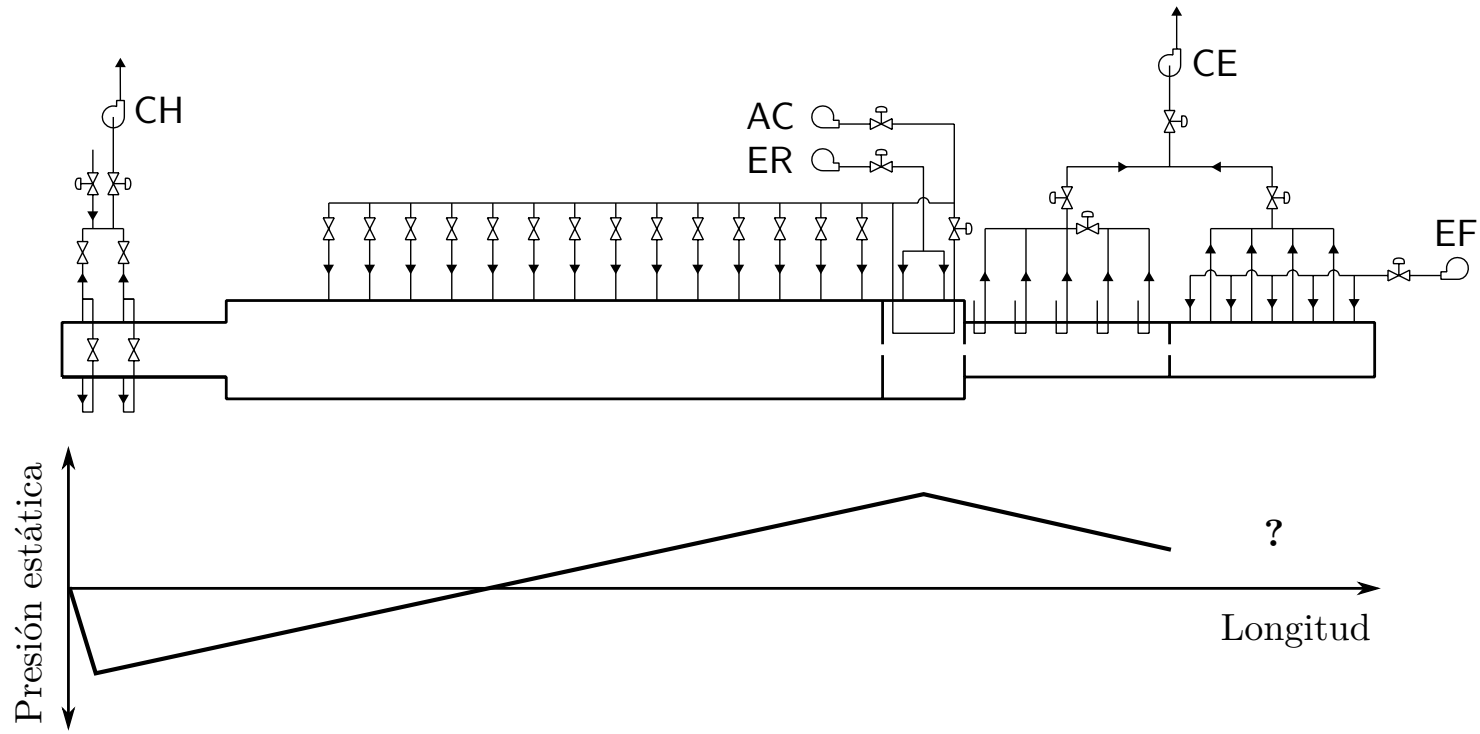

Figura 1.8. Entradas y salidas de gases en el horno de rodillos y curva de presión típica. 
La existencia de un punto de máxima presión estática sugiere la circulación de gases en dos sentidos opuestos: hacia la entrada del horno, donde se sitúa la chimenea de humos, y hacia la salida, donde está la chimenea de enfriamiento. Es conveniente que el máximo se encuentre en la zona de enfriamiento ya que, si se sitúa en la zona de cocción, parte de los gases de combustión se dirigirán a la zona de enfriamiento. Esta situación es claramente desfavorable desde el punto de vista energético. Por el contrario, si el máximo se localiza en el enfriamiento rápido, se incorporará aire de enfriamiento a la corriente de humos. Normalmente en el calentamiento se opera con un exceso de aire importante, para obtener una atmósfera lo suficientemente rica en oxígeno como para que tengan lugar las reacciones de oxidación de la materia orgánica presente en la composición cerámica. La incorporación de aire de enfriamiento, calentado con el calor sensible de las piezas, a la zona de calentamiento puede pues resultar beneficiosa, en tanto en cuanto se reduzca consecuentemente el exceso de aire aportado en los quemadores [19].

\subsubsection{Sistemas de control}

Los hornos de rodillos están dotados de sistemas de control automático. El más notable es el control de temperatura que involucra la medida de la temperatura de los gases en posiciones estratégicas a lo largo del horno. La curva de temperatura se especifica estableciendo los valores de consigna para estas temperaturas. Los dispositivos de medida suelen ser termopares de tipo $\mathrm{K}$ o $\mathrm{S}$, según la temperatura a la que estarán expuestos. En el calentamiento, cada anillo de quemadores cuenta con un termopar asociado situado en una posición representativa. En base a la temperatura registrada, el caudal de gas natural en el anillo se ajusta automáticamente. Suele haber un termopar adicional en la zona de aspiración de humos pero que no cuenta con un actuador asociado. El enfriamiento se controla con un número mucho más limitado de puntos de medida. Habitualmente, se emplea un único termopar por etapa de enfriamiento.

En ocasiones el ventilador encargado de la extracción de humos dispone de un variador de frecuencia que regula automáticamente la velocidad de giro de los álabes para mantener constante la presión estática en el interior del horno. Los hornos modernos también disponen de un sistema de control que modula el caudal total de aire comburente actuando sobre el correspondiente ventilador de impulsión. Este control es de gran utilidad pues amortigua las oscilaciones de temperatura en interrupciones ocasionales de la producción. Ahora bien, la regulación del aire en cada quemador es básicamente manual.

\subsubsection{Balance global de energía}

El calor liberado por la combustión de gas natural en los quemadores del horno acaba, en última instancia, siendo disipado de diversas maneras; por ejemplo, en forma de energía interna de varias corrientes (figura 1.9).

La energía liberada en la combustión hace que los gases resultantes alcancen la temperatura especificada. Los gases de combustión, en su trayecto hacia la chimenea de humos, transfieren parte de su energía a las baldosas y a las paredes. Puesto que este trayecto no es lo suficientemente largo como para ser considerado infinito a efectos de transferencia de energía, los humos retienen parte de la energía aportada, que acaba siendo evacuada por la chimenea. 


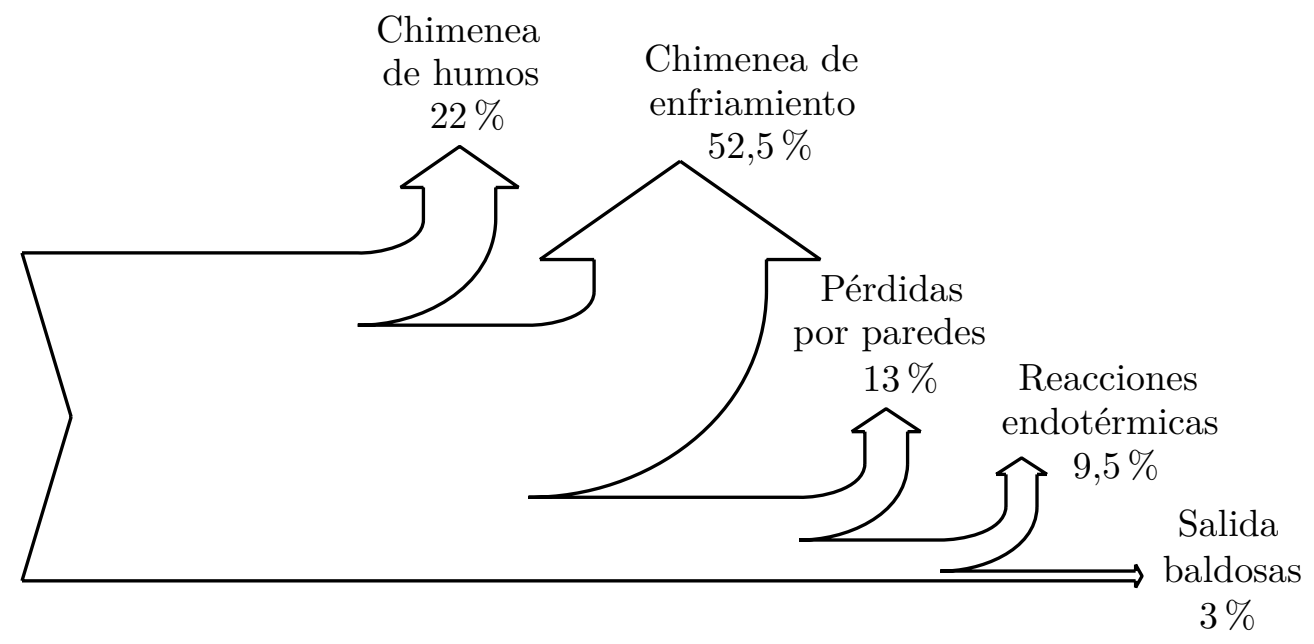

Figura 1.9. Diagrama de Sankey para un horno sin recuperación de calor [14].

El calor transmitido a las paredes es, en parte, conducido a su través para ser subsiguientemente transferido al entorno, constituyendo una fuente de pérdidas de energía. Pérdidas de naturaleza similar se producen en el trasiego de corrientes de gases calientes.

De entre las reacciones químicas que acontecen en el seno de las baldosas, las más significativas desde el punto de vista térmico son endotérmicas [20,21]. Por consiguiente, es necesario invertir una cierta cantidad de energía para que éstas tengan lugar. Esta energía se corresponde con la modificación en la entalpía de las baldosas al pasar de crudas a cocidas. Puesto que se trata de una modesta fracción del calor total aportado, suele argumentarse que la operación de cocción es extremadamente ineficiente [13,16, 22], aunque quizás este razonamiento sea un tanto simplista [23].

Las baldosas abandonan la zona de calentamiento prácticamente a la temperatura máxima de cocción. Este calor se transfiere, principalmente, al aire empleado en el enfriamiento, aunque también a las paredes que las rodean. Las baldosas abandonan el horno a una temperatura ligeramente superior a la temperatura ambiente y, en consecuencia, existe un flujo de calor sensible asociado.

Como puede observarse, una parte importante de las salidas de energía es el aire caliente procedente del enfriamiento. Este hecho no ha pasado inadvertido a los fabricantes de hornos, quienes han introducido diversos sistemas para recuperar esta energía. Un método de recuperación muy común consiste en precalentar el aire empleado como comburente mediante un intercamiador de calor, basado en un haz de tubos, que se sitúa en el enfriamiento rápido. El aire puede proceder del ambiente o de la chimenea de enfriamiento. Para obtener una mayor eficiencia, en ocasiones se instalan dos chimeneas de enfriamiento de manera que pueda separarse el aire de enfriamiento indirecto (o incluso el del enfriamiento rápido), más caliente, del aire de enfriamiento final. Dependiendo del sistema empleado, puede conseguirse que el aire suministrado a los quemadores alcance unos $100-200^{\circ} \mathrm{C}$.

Otra opción de recuperación de calor consiste en aprovechar el aire de enfriamiento, que esencialmente consiste en aire caliente exento de contaminantes, en los secaderos. Por ejemplo, algunos hornos disponen de un secadero túnel de vagonetas acoplado a la entrada del horno que utiliza aire de enfriamiento para el secado. Mediante este sistema se elimina 
el agua que hayan podido adquirir las piezas en el esmaltado, con lo que se previenen las explosiones de las piezas en la entrada del horno derivadas de la evaporación violenta de la misma [24].

El aprovechamiento de la energía contenida en los humos presenta mayor dificultad, ya que la presencia de contaminantes $\left(\mathrm{SO}_{2}, \mathrm{HCl}, \mathrm{HF}\right.$, entre otros) impide la recuperación directa de los gases y exige el uso de intercambiadores de calor [22,25].

\subsection{Mecanismos de transferencia de energía en hornos de rodillos}

\subsubsection{Transferencia entre los gases y las superficies interiores}

Está generalmente admitido que existen dos mecanismos por los cuales se produce la transferencia de energía entre los gases y las superficies interiores del horno: convección y radiación $[26]$.

La convección tiene lugar como consecuencia del contacto entre los gases y las superficies interiores del horno. La convección está asociada a un flujo de materia desde la superficie hacia el seno de los gases (o viceversa). Se distingue entre convección natural y convección forzada, que pueden encontrarse solapadas. En el caso de la convección natural, el flujo de materia se debe exclusivamente a variaciones de densidad que resultan de diferencias de temperatura, mientras que en la convección forzada es la acción de una fuerza externa (diferencia de presión) la que pone en movimiento el fluido. En el interior de los hornos de rodillos la convección forzada predomina, por la turbulencia generada por los quemadores y por el régimen turbulento de circulación de los gases en general.

El flujo de calor $\dot{Q}$ que alcanza a una superficie por convección puede describirse mediante la ley del enfriamiento de Newton:

$$
\dot{Q}=A h_{C}\left(T-T_{g}\right)
$$

donde $A$ es el área de la superficie considerada, $h_{C}$ es el coeficiente individual de transferencia de energía por convección, $T$ es la temperatura de la superficie y $T_{g}$ es la temperatura en el seno de los gases con los que está en contacto la superficie.

La transmisión de calor por radiación se produce sin que sea necesaria la existencia de un medio material que transporte esta energía, a diferencia de lo que ocurría con la convección. Por este motivo, las superficies pueden intercambiar energía por este mecanismo sin que sea necesario un contacto físico entre ellas. Además, ciertos gases, como el dióxido de carbono $\left(\mathrm{CO}_{2}\right)$ y el vapor de agua $\left(\mathrm{H}_{2} \mathrm{O}\right)$, pueden absorber y emitir radiación térmica. Esto significa que, en el horno de rodillos, los humos (pero no el aire de enfriamiento) también participan en el intercambio de energía radiante.

El flujo de calor por radiación depende de la diferencia de temperaturas absolutas elevadas a la cuarta potencia:

$$
\dot{Q}=A \varepsilon_{R} \sigma\left(T^{4}-T_{g}^{4}\right)
$$


donde $\varepsilon_{R}$ es una «emisividad resultante» y $\sigma$ es la constante de Stefan-Boltzmann. En ocasiones, por analogía con la ecuación (1.1), la expresión (1.2) se escribe en la forma:

$$
\dot{Q}=A h_{R}\left(T-T_{g}\right)
$$

siendo:

$$
h_{R}=\varepsilon_{R} \sigma\left(T^{2}+T_{g}^{2}\right)\left(T+T_{g}\right)
$$

Suele considerarse que la temperatura de los gases en la proximidad de la superficie, $T_{g}$, determina completamente el flujo radiativo que alcanza a dicha superficie, de acuerdo con la ecuación (1.2) [27-29], lo que no es estrictamente cierto. Asimismo, el concepto de emisividad resultante es también una simplificación. En §1.6 se presenta un tratamiento más riguroso del intercambio de energía radiante.

Las elevadas temperaturas que se dan en el horno hacen que la radiación sea el principal mecanismo de transferencia de energía entre superficies y gases en la zona de calentamiento [27]. Por el contrario, en la zona de enfriamiento, el aire no interviene significativamente en el intercambio radiativo, puesto que no contiene cantidades sustanciales de especies absorbentes. En consecuencia, en la zona de enfriamiento sólo se produce intercambio de radiación térmica entre superficies. La transferencia de calor entre las superficies y el aire de enfriamiento se produce básicamente por convección.

\subsubsection{Transferencia a través de las paredes y disipación al entorno}

Las paredes transmiten el calor a través de su seno por conducción, que involucra vibraciones reticulares y, en el caso de sólidos metálicos, migración de electrones libres. Para analizar la conducción a través de las paredes del horno, se suele considerar que éstas son láminas de extensión infinita y espesor finito [13]. Bajo esta aproximación, la ley de Fourier de la conducción puede escribirse [30]:

$$
\dot{Q}=-A \lambda \frac{\partial T}{\partial x}
$$

en la que $T$ es la temperatura en una sección cualquiera de la pared, $\lambda$ es la conductividad térmica del sólido y $x$ es la dirección en la que se produce la transmisión.

En estado estacionario, si $\lambda$ no depende de la temperatura, el perfil de temperaturas según (1.5) resulta ser lineal:

$$
\dot{Q}=A \lambda \frac{T_{i}-T_{o}}{\ell}
$$

donde $T_{i}$ y $T_{o}$ son las temperaturas en las superficie interior y exterior de la pared, respectivamente, $\mathrm{y} \ell \mathrm{su}$ espesor.

El flujo de calor que abandona el horno a través de las paredes se transfiere al ambiente (a temperatura $T_{\infty}$ ) por convección y radiación. Resulta conveniente englobar estos mecanismos acoplados en una ecuación de la forma:

$$
\dot{Q}=A U\left(T_{i}-T_{\infty}\right)
$$



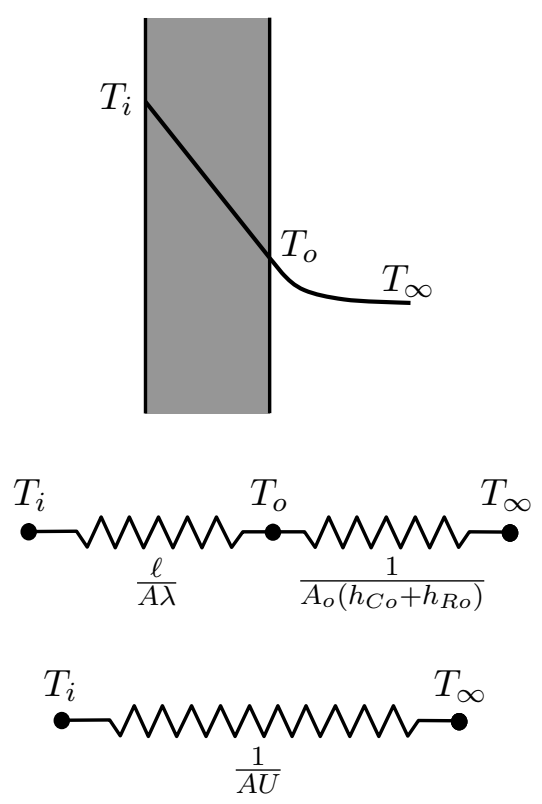

Figura 1.10. Diagrama de Oppenheim para las pérdidas por paredes.

siendo $U$ el coeficiente global de transmisión de calor. Este coeficiente global incorpora los distintos mecanismos implicados (figura 1.10).

De acuerdo con el diagrama de la figura 1.10, se tiene:

$$
\frac{T_{i}-T_{o}}{\frac{\ell}{A \lambda}}=\frac{T_{o}-T_{\infty}}{\frac{1}{A_{o}\left(h_{C o}+h_{R o}\right)}}=\frac{T_{i}-T_{\infty}}{\frac{1}{A U}}
$$

y, por tanto:

$$
\frac{1}{U}=\frac{\ell}{\lambda}+\frac{A / A_{o}}{h_{C o}+h_{R o}}
$$

En este caso la convección es principalmente natural, por lo que $h_{C o}$ puede calcularse a partir de correlaciones empíricas que relacionan el módulo de Nusselt con los módulos de Rayleigh y de Prandtl. El número de Nusselt se define como:

$$
\mathrm{Nu}=\frac{h_{C o} L}{\lambda_{a}}
$$

donde $L$ es una longitud característica y $\lambda_{a}$ es la conductividad térmica del aire. El módulo de Prandtl viene dado por:

$$
\operatorname{Pr}=\frac{\nu_{a}}{\alpha_{a}}
$$

siendo $\nu_{a}$ la viscosidad cinemática del fluido (aire, en este caso) y $\alpha_{a}$ su difusividad térmica. El módulo de Rayleigh se calcula mediante:

$$
\mathrm{Ra}=\frac{g \beta\left|T_{o}-T_{\infty}\right| L^{3}}{\nu_{a} \alpha_{a}}
$$


donde $g$ es la aceleración de la gravedad y $\beta$ es el coeficiente de expansión cúbica, que para gases ideales:

$$
\beta=\frac{1}{V}\left(\frac{\partial V}{\partial T}\right)_{p}=\frac{1}{T}
$$

El coeficiente individual de transferencia por radiación puede calcularse mediante:

$$
h_{R o}=\sigma \varepsilon_{o}\left(T_{o}^{2}+T_{\infty}^{2}\right)\left(T_{o}+T_{\infty}\right)
$$

donde $\varepsilon_{o}$ es la emisividad de la superficie exterior.

\subsubsection{Transferencia en el interior de las piezas}

En el interior de las piezas el mecanismo fundamental de transmisión de calor es también la conducción. La temperatura de las piezas obviamente evoluciona durante la cocción luego, en este caso, no se establecerá un estado estacionario. La ecuación que permite calcular la evolución con el tiempo de la distribución interna de temperaturas adopta la forma [27-29]:

$$
\frac{\partial T_{b}}{\partial t}=\alpha_{b} \nabla^{2} T_{b}
$$

donde $\alpha_{b}$ es la difusividad térmica aparente de las baldosas:

$$
\alpha_{b}=\frac{\lambda_{b}}{\rho_{b}\left(c_{p b}+6 \sum_{k} \frac{w_{k} \Delta_{r} H_{k}^{\ominus}}{T_{k}^{+}-T_{k}^{-}}\left\langle\Theta_{k}\left(1-\Theta_{k}\right)\right\rangle\right)}
$$

siendo $\lambda_{b}$ la conductividad térmica de las baldosas, $\rho_{b}$ su densidad aparente y $c_{p b}$ su capacidad calorífica media. El segundo término del denominador de (1.16) tiene en cuenta el cambio térmico asociado a las reacciones químicas que experimenta la composición cerámica. Se supone que la $k$-ésima reacción química tiene lugar progresivamente entre dos temperaturas $T_{k}^{-}$y $T_{k}^{+}$, liberando en el proceso el calor de reacción $\Delta_{r} H_{k}^{\ominus}$. El calor de reacción está expresado en base a la masa de un componente de referencia cuya fracción másica inicial en la composición cerámica se denota por $w_{k}$. El parámetro $\Theta_{k}$ se define como:

$$
\Theta_{k}=\frac{T_{b}-T_{k}^{-}}{T_{k}^{+}-T_{k}^{-}}
$$

y los símbolos $\langle\bullet\rangle$ representan los corchetes de Macaulay (definidos en §A.1.4).

\subsubsection{Transferencia por contacto entre los rodillos y las piezas}

Los rodillos son los encargados de la sustentación y desplazamiento de las baldosas y, por tanto, existe un contacto físico entre ambos. Como resultado de este contacto, y del hecho que estos elementos del horno son susceptibles de encontrarse a temperaturas muy distintas, cabe la posibilidad de que existan mecanismos adicionales de transferencia de energía entre 
ellos. Se examinarán dos teorías para la descripción de este fenómeno. Ambas teorías son en principio aplicables a cualquier contacto móvil, pero aquí se particularizarán al caso de la transferencia entre los rodillos y las baldosas, para lo que se utilizará el subíndice $r$ para referirse a los rodillos y $b$ para las baldosas.

En primer lugar, se tiene la teoría de Bejan [31], la cual se basa en que entre dos sólidos elásticos en contacto, para los que existe una fuerza que tiende a unirlos (por ejemplo, un sólido es sustentado por el otro), se desarrollará un área de contacto hertziano. Este área de contacto será, de acuerdo con la teoría de Hertz [32], un elipsoide de semiejes $a$ y $b$. Bejan considera a los sólidos en contacto como semiinfinitos a temperatura uniforme, salvo en la interfase entre ellos, donde se desarrolla un perfil de temperaturas gobernado por la ecuación de Fourier de la conducción. La solución analítica de dicha ecuación es:

$$
\frac{\dot{Q}}{T_{r}-T_{b}}=2,789 \frac{b \sqrt{u a}}{\frac{1}{\sqrt{\rho_{b} c_{p b} \lambda_{b}}}+\frac{1}{\sqrt{\rho_{r} c_{p r} \lambda_{r}}}}
$$

donde $u$ es la velocidad lineal de rotación de los rodillos (y de avance de las baldosas) y los otros símbolos han sido definidos previamente.

La teoría de Bejan no parece ser de aplicación al caso de la transferencia entre las baldosas y los rodillos. Aún suponiendo que sus superficies son perfectamente lisas, que no es el caso, la carga que mantiene unidos a estos elementos - el peso de las baldosas - generaría un contacto hertziano de extensión poco relevante a efectos de transferencia de energía. En efecto, el área de contacto será prácticamente puntual.

Specht y colaboradores [33-35] admiten que el contacto entre rodillos y láminas de material soportadas por éstos es puntual, luego no habrá transferencia de energía por conducción a través de los materiales. En su lugar, estos autores asumen que existe una capa límite en la cual se produce el transporte de calor por conducción molecular unidireccional a través del gas. Mediante esta consideración, llegan a la siguiente expresión para un coeficiente local de transmisión de calor:

$$
h_{x}=\frac{\lambda_{g}}{R-\sqrt{R^{2}-x^{2}}+\Lambda}
$$

en la que $\lambda_{g}$ es la conductividad térmica del gas, $\Lambda$ es un recorrido libre medio molecular modificado, $R$ es el radio de los rodillos y $x$ es la distancia entre la línea de contacto y un punto en la superficie de las baldosas. El coeficiente individual de transmisión de calor se obtiene por integración de la ecuación (1.19).

El modelo de Specht y colaboradores parece reproducir adecuadamente los resultados experimentales [35]. No obstante, para que así sea, introducen una distancia adicional en el denominador de (1.19), que interpretan como debida a la rugosidad de los materiales. $\mathrm{Al}$ introducir esta distancia, el coeficiente individual de transferencia desciende drásticamente. Una rugosidad de $500 \mu \mathrm{m}$ (como el relieve del reverso o «costilla» de las baldosas) hace que sea muy inferior a $25 \mathrm{~W} \mathrm{~m}^{-2} \mathrm{~K}^{-1}$, valor que cabría esperar para la transferencia por convección entre los gases y las superficies [36]. Así pues, los resultados de Specht y colaboradores sugieren que la transferencia de calor por contacto entre las baldosas y los rodillos no es muy importante. 


\subsection{El método zonal}

Para analizar la transferencia de energía en el horno de rodillos, cuando se considera éste en su conjunto, es necesario recurrir a modelos más rigurosos que los considerados hasta el momento; en particular, en lo que se refiere a la transferencia de radiación térmica.

El intercambio de radiación térmica en presencia de un gas absorbente es un fenómeno complejo. La transferencia de energía radiante bajo estas circunstancias se describe mediante una ecuación integro-diferencial [37]. Ello se debe a que, cuando se plantea un balance de energía a un volumen de control infinitesimal, éste puede emitir y recibir radiación térmica desde todas las direcciones. La naturaleza integro-diferencial de la ecuación de conservación de la energía radiante propicia que no puedan utilizarse directamente las técnicas habituales de discretización de ecuaciones en derivadas parciales sin recurrir a aproximaciones adicionales.

Una posible aproximación consiste en dividir el ángulo sólido que rodea al elemento de volumen en varias porciones en las cuales se supone que el flujo de radiación es constante. En base a esta hipótesis pueden formularse modelos de dos, cuatro o seis flujos, para resolver problemas de una, dos o tres dimensiones, respectivamente [38].

Los métodos de Monte Carlo evitan tener que utilizar simplificaciones como la indicada [39]. En estos métodos, se simula la emisión y absorción de fotones individuales. Cada fotón tiene una determinada probabilidad de ser absorbido por una superficie o un volumen de gas. Así, mediante la generación de números aleatorios, puede estudiarse la historia de un gran número de fotones emitidos aleatoriamente por los elementos del sistema. Si el número de fotones es lo suficientemente elevado, el método converge a la solución exacta.

Aunque se suele considerar al método de Monte Carlo como una técnica genuina de resolución de la ecuación de conservación de la energía radiante, frecuentemente su uso se limita a la determinación de las áreas de intercambio que surgen en el método zonal [40]. El método zonal, que se desarrolla seguidamente, fue introducido por Hottel y Cohen [41], basándose en el trabajo que constituyó la tesis doctoral de Cohen [42]. Posteriormente, fue revisado por Sarofim [43] y Hottel y Sarofim [44]. En este método, se descompone el sistema en una serie de volúmenes y superficies — zonas - lo suficientemente pequeñas como para ser consideradas isotermas. El método zonal permite calcular los flujos radiativos entre estas zonas. El cálculo involucra determinar sucesivamente tres conjuntos de áreas de intercambio, las cuales se definen a continuación.

\subsection{1. Áreas de intercambio directo}

En esta sección se utilizarán los conceptos de «superficies negras» y «gases grises». Las superficies negras absorben toda radiación incidente y la radiación que emiten no depende de la dirección de emisión (emisor difuso). Los gases grises absorben la radiación térmica según la ley de Lambert-Beer. Realmente, ni las las superficies del horno son negras ni los gases de combustión son grises. El tratamiento de superficies grises y gases reales se abordará en las secciones siguientes.

Considérense dos superficies negras isotermas $A_{i}$ y $A_{j}$, de geometría arbitraria, que intercambian radiación térmica a través de un gas gris con coeficiente de absorción $K$ (figura 1.11). 


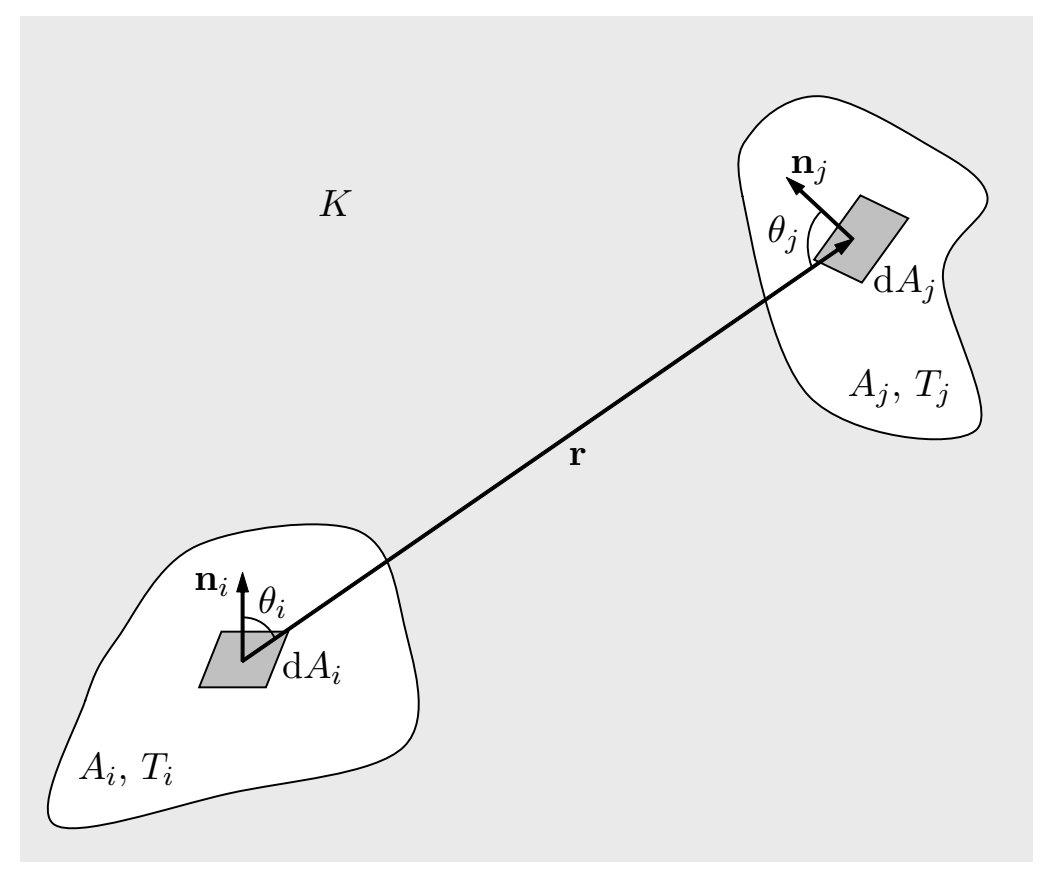

Figura 1.11. Intercambio de radiación térmica entre dos superficies elementales en presencia de un gas gris.

El flujo de energía radiante emitido por el elemento de superficie $\mathrm{d} A_{i}$ que alcanza directamente a $\mathrm{d} A_{j}$ se calcula por [45]:

$$
\mathrm{d} \dot{Q}_{i \rightarrow j}=\frac{\cos \theta_{i} \cos \theta_{j}}{\pi r^{2}} \mathrm{e}^{-K r} E_{i} \mathrm{~d} A_{i} \mathrm{~d} A_{j}
$$

Esta ecuación consta de varios factores:

$\cos \theta_{i} \mathrm{~d} A_{i} \quad$ es la proyección del elemento de superficie $\mathrm{d} A_{i}$ en la dirección en la que se produce el intercambio.

$\frac{\cos \theta_{j}}{\pi r^{2}} \mathrm{~d} A_{j} \quad$ es el ángulo sólido cubierto por $\mathrm{d} A_{j}$ cuando éste es observado desde $\mathrm{d} A_{i}$.

$\mathrm{e}^{-K r} \quad$ es la fracción de energía transmitida (no absorbida) a través del gas gris (transmitancia), según la ley de Lambert-Beer.

$E_{i} \quad$ es el poder emisivo del cuerpo negro a la temperatura de la superficie $A_{i}$, que viene dado por la ley de Stefan-Boltzmann:

$$
E_{i}=\sigma T_{i}^{4}
$$

donde $\sigma$ es la constante de Stefan-Boltzmann y $T_{i}$ es la temperatura absoluta de la superficie $A_{i}$.

El flujo total de radiación que abandona la superficie $A_{i}$ para ser interceptado por la superficie $A_{j}$ se obtiene integrando la ecuación (1.20):

$$
\dot{Q}_{i \rightarrow j}=E_{i} \iint_{A_{j}} \iint_{A_{i}} \frac{\cos \theta_{i} \cos \theta_{j}}{\pi r^{2}} \mathrm{e}^{-K r} \mathrm{~d} A_{i} \mathrm{~d} A_{j}
$$


A la integral de esta expresión se le denomina área de intercambio directo (direct exchange area, DEA) y se le denota:

$$
\overline{s_{i} s_{j}}=\iint_{A_{j}} \iint_{A_{i}} \frac{\cos \theta_{i} \cos \theta_{j}}{\pi r^{2}} \mathrm{e}^{-K r} \mathrm{~d} A_{i} \mathrm{~d} A_{j}
$$

Las DEA dependen únicamente de la geometría del sistema y de las propiedades radiantes del gas gris. De la definición de $\overline{s_{i} s_{j}}$ se desprende que:

$$
\overline{s_{i} s_{j}}=\overline{s_{j} s_{i}}
$$

relación que se conoce como propiedad de reciprocidad.

$\mathrm{Si}$ el gas que se encuentra entre ambas superficies es gris, éste también participa en el intercambio de energía radiante. Así, existe un flujo de calor entre las superficies y el gas:

$$
\mathrm{d} \dot{Q}_{i \rightarrow j}=\frac{\cos \theta_{i} K}{\pi r^{2}} \mathrm{e}^{-K r} E_{i} \mathrm{~d} A_{i} \mathrm{~d} V_{j}
$$

En los gases, la absorción de radiación es un fenómeno volumétrico, en lugar de superficial, por lo que el factor $\cos \theta_{j} \mathrm{~d} A_{j}$ se ve sustituido por $K \mathrm{~d} V_{j}$, en el que $\mathrm{d} V_{j}$ es un elemento infinitesimal de volumen. La integración de la ecuación (1.25) sugiere la definición del DEA entre una superficie y un volumen de gas:

$$
\overline{s_{i} g_{j}}=\iiint_{V_{j}} \iint_{A_{i}} \frac{\cos \theta_{i} K}{\pi r^{2}} \mathrm{e}^{-K r} \mathrm{~d} A_{i} \mathrm{~d} V_{j}
$$

Las DEA entre superficies y gases también obedecen una relación de reciprocidad similar a (1.24):

$$
\overline{s_{i} g_{j}}=\overline{g_{j} s_{i}}
$$

Del mismo modo en el que se divide un sistema en superficies isotermas, el gas también puede considerarse compuesto de diversos volúmenes isotermos. Estos volúmenes de gas son, por supuesto, también susceptibles de intercambiar radiación térmica:

$$
\mathrm{d} \dot{Q}_{i \rightarrow j}=\frac{K^{2}}{\pi r^{2}} \mathrm{e}^{-K r} E_{i} \mathrm{~d} V_{i} \mathrm{~d} V_{j}
$$

pudiéndose definir el DEA entre dos volúmenes de gas $V_{i}$ y $V_{j}$ :

$$
\overline{g_{i} g_{j}}=\iiint_{V_{j}} \iiint_{V_{i}} \frac{K^{2}}{\pi r^{2}} \mathrm{e}^{-K r} \mathrm{~d} V_{i} \mathrm{~d} V_{j}
$$

La relación de reciprocidad es en este caso:

$$
\overline{g_{i} g_{j}}=\overline{g_{j} g_{i}}
$$

Usualmente, los equipos a calcular constituyen un sistema cerrado. Es decir, se trata de cerramientos de los cuales no puede escapar la radiación. Estos cerramientos pueden estar compuestos de $m$ superficies y $n$ volúmenes de geometría arbitraria. La conservación de la energía en el sistema cerrado implica que:

$$
\begin{aligned}
& \sum_{j=1}^{m} \overline{s_{i} s_{j}}+\sum_{j=1}^{n} \overline{s_{i} g_{j}}=A_{i} \\
& \sum_{j=1}^{m} \overline{g_{i} s_{j}}+\sum_{j=1}^{n} \overline{g_{i} g_{j}}=4 K V_{i}
\end{aligned}
$$




\subsection{2. Áreas de intercambio total}

El comportamiento frente a la radiación térmica de las superficies interiores de los hornos, incluidas las de las baldosas cerámicas, se asemeja mucho al de las superficies negras. Las superficies de estos materiales absorben y emiten radiación con independencia de la longitud de onda y la dirección de la radiación incidente. No obstante, estas superficies absorben solamente una parte de la radiación incidente, siendo la fracción complementaria reflejada de manera difusa. Las superficies que responden a este comportamiento se llaman superficies grises difusas.

Las superficies grises emiten una fracción, denominada emisividad $\varepsilon$, de la radiación que emitiría un cuerpo negro a su misma temperatura. La ley de Kirchhoff establece que, en equilibrio térmico, esta fracción coincide con la fracción de la radiación incidente que la superficie es capaz de absorber. La fracción restante o reflectancia $\varrho$ es reflejada en todas las direcciones por igual. Queda entonces claro que se verifica:

$$
\varepsilon+\varrho=1
$$

Así pues, en un cerramiento de superficies grises, parte de la energía radiante será absorbida por las superficies, mientras que el resto será reflejado. La energía reflejada puede ser de nuevo parcialmente absorbida y parcialmente reflejada, repitiéndose el proceso indefinidamente.

Hottel y Cohen [41] englobaron el efecto neto del proceso indicado en las áreas de intercambio total (total exchange areas, TEA). Si bien estas cantidades simplifican enormemente la formulación de los flujos netos radiativos, el método indicado para su cálculo era implícito y poco sistemático. Noble [46] solventó esta dificultad presentando las fórmulas explícitas para su cálculo que se recogen a continuación.

Las fórmulas de Noble son expresiones matriciales que implican la organización de las DEA en cuatro matrices: $[\overline{\mathbf{s s}}],[\overline{\mathbf{s g}}],[\overline{\mathbf{g s}}]=[\overline{\mathbf{s g}}]^{T}$ y $[\mathbf{g g}]$. A partir de ellas, y de las propiedades de las superficies grises, pueden calcularse las matrices de TEA:

$$
\begin{aligned}
{[\overline{\mathbf{S S}}] } & =\operatorname{diag}\left(A_{i} \varepsilon_{i}\right) \cdot[\mathbf{R}] \cdot[\overline{\mathbf{s S}}] \cdot \operatorname{diag}\left(\varepsilon_{i}\right) \\
{[\overline{\mathbf{S G}}] } & =[\overline{\mathbf{G S}}]^{T}=\operatorname{diag}\left(A_{i} \varepsilon_{i}\right) \cdot[\mathbf{R}] \cdot[\overline{\mathbf{s g}}] \\
{[\overline{\mathbf{G G}}] } & =[\overline{\mathbf{g s}}] \cdot \operatorname{diag}\left(\varrho_{i}\right) \cdot[\mathbf{R}] \cdot[\overline{\mathbf{s g}}]+[\overline{\mathbf{g g}}]
\end{aligned}
$$

en las que:

$$
[\mathbf{R}]=\left(\operatorname{diag}\left(A_{i}\right)-[\overline{\mathbf{s s}}] \cdot \operatorname{diag}\left(\varrho_{i}\right)\right)^{-1}
$$

y $\operatorname{diag}(\bullet)$ hace referencia a la matriz diagonal cuyos elementos son los argumentos de la función.

A partir de las TEA, pueden expresarse los flujos netos radiativos que alcanzan a las zonas del siguiente modo:

$$
\dot{Q}_{i}=\sum_{j=1}^{m} \overline{S_{j} S_{i}} E_{j}+\sum_{j=1}^{n} \overline{G_{j} S_{i}} E_{g, j}-A_{i} \varepsilon_{i} E_{i}
$$


para superficies, y:

$$
\dot{Q}_{g, i}=\sum_{j=1}^{m} \overline{S_{j} G_{i}} E_{j}+\sum_{j=1}^{n} \overline{G_{j} G_{i}} E_{g, j}-4 K V_{i} E_{g, i}
$$

para los gases.

Las ecuaciones (1.38) y (1.39) implican las siguientes ecuaciones de conservación:

$$
\begin{gathered}
\sum_{j=1}^{m} \overline{S_{j} S_{i}}+\sum_{j=1}^{n} \overline{G_{j} S_{i}}=A_{i} \varepsilon_{i} \\
\sum_{j=1}^{m} \overline{S_{j} G_{i}}+\sum_{j=1}^{n} \overline{G_{j} G_{i}}=4 K V_{i}
\end{gathered}
$$

Nótese que el cálculo de las TEA involucra la inversión de una matriz que es susceptible de contener muchos elementos. Existen algoritmos que evitan invertir dicha matriz mediante los métodos algebraicos convencionales [47,48].

\subsection{3. Áreas de flujo dirigido}

La emisión y absorción de radiación térmica por parte de los gases de combustión es principalmente debida a transiciones en los estados de energía vibracionales y rotacionales de las moléculas de $\mathrm{H}_{2} \mathrm{O}$ y $\mathrm{CO}_{2}$. Las mezclas de estos gases no se comportan como gases grises. La emisividad total (integrada en todo el espectro) de estas mezclas depende de la temperatura, de la presión total y de las presiones parciales de $\mathrm{H}_{2} \mathrm{O}$ y de $\mathrm{CO}_{2}$.

Si bien la emisividad total de los gases reales $\varepsilon_{g}$ no puede expresarse en términos de un solo gas gris, ésta sí que puede describirse mediante una suma ponderada de gases grises (weighted sum of grey gases, WSGG):

$$
\varepsilon_{g} \simeq \sum_{k=0}^{q} a_{k}\left(T_{g}\right)\left(1-\mathrm{e}^{-\kappa_{k} p_{R} r}\right)
$$

en la que $p_{R}$ es la suma de las presiones parciales de $\mathrm{H}_{2} \mathrm{O}$ y de $\mathrm{CO}_{2}$, y $a_{k}\left(T_{g}\right)$ y $\kappa_{k}$ son parámetros experimentales. Los pesos dependen de la temperatura de la mezcla de gases y verifican:

$$
\sum_{k=0}^{q} a_{k}\left(T_{g}\right)=1
$$

La WSGG también fue una contribución de Hottel y Cohen [41], quienes ya apuntaban que con tan sólo unos pocos gases grises y un gas claro $(K=0)$ debería obtenerse una precisión suficiente.

Es frecuente considerar que los pesos varían linealmente con la temperatura:

$$
a_{k}(T)=b_{0 k}+b_{1 k} T
$$




\begin{tabular}{cccc}
$k$ & $b_{0 k}$ & $b_{1 k} \cdot 10^{6}\left(\mathrm{~K}^{-1}\right)$ & $\kappa_{k}\left(\mathrm{~atm}^{-1} \mathrm{~m}^{-1}\right)$ \\
\hline 0 & 0,437 & 71,3 & 0 \\
\hline 1 & 0,390 & $-5,2$ & 1,88 \\
\hline 2 & 0,173 & $-66,1$ & 68,8 \\
\hline
\end{tabular}

Tabla 1.2. Coeficientes para el modelo de dos gases grises y un gas claro de Truelove [49]. $p=1$ atm. $p_{\mathrm{H}_{2} \mathrm{O}} / p_{\mathrm{CO}_{2}}=2$.

En la tabla 1.2 se muestran los coeficientes del modelo WSGG de Truelove [49] de dos gases grises y un gas claro. Este autor también presentó un modelo de tres gases grises y un gas claro, pero algunos autores [50] señalan que la mejora no es muy sustancial.

Por los argumentos expuestos por Sarofim [43], los pesos para la absortancia y la transmitancia deben evaluarse a la temperatura del emisor, por lo que pudieron haberse definido las DEA entre superficies, por ejemplo, de la siguiente manera alternativa:

$$
\overline{s_{i} s_{j}}=\sum_{k=0}^{q} a_{k}\left(T_{i}\right) \iint_{A_{j}} \iint_{A_{i}} \frac{\cos \theta_{i} \cos \theta_{j}}{\pi r^{2}} \mathrm{e}^{-\kappa_{k} p_{R} r} \mathrm{~d} A_{i} \mathrm{~d} A_{j}
$$

No obstante, puesto que los pesos no dependen de la geometría del sistema, es más práctico retardar la ponderación hasta la formulación de los flujos netos radiativos.

Las áreas de flujo dirigido (directed flux areas, DFA) se definen como:

$$
\begin{aligned}
\overrightarrow{S_{i} S_{j}} & =\sum_{k=0}^{q} a_{k}\left(T_{i}\right) \overline{S_{i} S_{j}}\left(K_{k}\right) \\
\overrightarrow{S_{i} G_{j}} & =\sum_{k=0}^{q} a_{k}\left(T_{i}\right) \overline{S_{i} G_{j}}\left(K_{k}\right) \\
\overrightarrow{G_{i} S_{j}} & =\sum_{k=0}^{q} a_{k}\left(T_{g, i}\right) \overline{G_{i} S_{j}}\left(K_{k}\right) \\
\overrightarrow{G_{i} G_{j}} & =\sum_{k=0}^{q} a_{k}\left(T_{g, i}\right) \overline{G_{i} G_{j}}\left(K_{k}\right)
\end{aligned}
$$

en las que las TEA se evalúan a partir de las DEA calculadas con los coeficientes de absorción $K_{k}=\kappa_{k} p_{R}$. El formalismo de las DFA permite expresar los flujos de energía radiante de una manera extraordinariamente compacta:

$$
\begin{aligned}
\dot{Q}_{i} & =\sum_{j=1}^{m} \overrightarrow{S_{j} S_{i}} E_{j}+\sum_{j=1}^{n} \overrightarrow{G_{j} S_{i}} E_{g, j}-A_{i} \varepsilon_{i} E_{i} \\
\dot{Q}_{g, i} & =\sum_{j=1}^{m} \overrightarrow{S_{j} G_{i}} E_{j}+\sum_{j=1}^{n} \overrightarrow{G_{j} G_{i}} E_{g, j}-4\left(\sum_{k=0}^{q} a_{k}\left(T_{g, i}\right) \kappa_{k}\right) p_{R} V_{i} E_{g, i}
\end{aligned}
$$

Debe notarse que en general $\overrightarrow{S_{j} S_{i}} \neq \overrightarrow{S_{i} S_{j}}, \overrightarrow{S_{j} G_{i}} \neq \overrightarrow{G_{i} S_{j}}$ y $\overrightarrow{G_{j} G_{i}} \neq \overrightarrow{G_{i} G_{j}}$, a no ser que $T_{j}=T_{i}, T_{j}=T_{g, i}$ o $T_{g, j}=T_{g, i}$, respectivamente. Queda claro el carácter direccional de las DFA y la importancia de la flecha en su notación. 
El método zonal permite calcular los flujos netos de radiación térmica entre las zonas en las que se divida un cerramiento de gases reales en superficies grises, mediante las ecuaciones (1.50) y (1.51). Dicho cálculo implica la determinación de las DFA, las cuales dependen de las TEA que, a su vez, se calculan a partir de las DEA. 


\section{Motivación, objetivos y alcance}

\subsection{Motivación}

La necesidad del estudio de la transferencia de energía en hornos monoestrato de rodillos para la cocción de baldosas cerámicas se justifica por múltiples factores, de diferente naturaleza.

El conocimiento de los fenómenos de transferencia de energía que tienen lugar en el horno de rodillos es esencial para diseñar, dimensionar, proyectar y construir el horno. Partiendo de unas especificaciones de diseño consistentes en unos requisitos de producción y una curva de cocción típica, deben establecerse las dimensiones del horno, decidirse el número de quemadores y la ubicación de los mismos, calcularse el diámetro de las conducciones y la potencia de los ventiladores, seleccionarse los materiales de todos los componentes, y realizarse los cálculos estructurales, entre otros muchos menesteres. Todas estas labores exigen una comprensión profunda de los procesos de transmisión de calor en el horno de rodillos.

Cuando el horno se encuentra en operación, el proceso debe estar bajo control, incluido cuando se emprende o interrumpe la producción, o en otras situaciones de carácter transitorio. Asimismo, el horno tiene que permitir modificar rápidamente el ciclo de cocción para adaptarse a la variedad de productos por la que se caracterizan las empresas fabricantes de baldosas cerámicas. Por lo tanto, es necesario instrumentar el horno para monitorizar las variables adecuadas en las posiciones oportunas e implantar sistemas de control automático. La respuesta del sistema está, por supuesto, gobernada por la transferencia de energía.

Debido a que en la operación de cocción es en la que el producto adquiere sus propiedades finales, para asegurar la calidad del mismo, es fundamental comprender la transmisión de calor que tiene lugar en el horno, ya que ésta determina la relación entre la curva de temperatura programada y aquella a la que en realidad quedan sometidas las baldosas.

Aparte de los factores eminentemente tecnológicos, cabe mencionar que la optimización de las condiciones de funcionamiento del horno de rodillos tiene asociados beneficios económicos. En un año de producción de un horno, se consume fácilmente más de un millón de euros en gas natural. Además, la tarifa del el gas natural está sujeta a la volatilidad propia de los combustibles fósiles, así como a gravámenes adicionales tales como la tasa al gas natural dictada por la Ley 15/2012 de medidas fiscales para la sostenibilidad energética. En un estudio encargado por la Comisión Europea [51], se constató que el precio del gas natural para las industrias fabricantes de baldosas cerámicas en el sur de Europa se vio incrementado un $36 \%$ en el periodo 2010-2014. El incremento de la tarifa del gas natural en los últimos años ha provocado una preocupación creciente entre los fabricantes de baldosas, quienes han visto cómo los costes de fabricación han ido en aumento por el incremento del precio de la energía. 
La racionalización del consumo de energía en la cocción de las baldosas cerámicas repercute positivamente tanto en la reducción de los costes de fabricación, como en la disminución de emisiones a la atmósfera. El gas natural es un combustible de origen fósil, por lo que su combustión da $\mathrm{CO}_{2}$ como producto. $\mathrm{El} \mathrm{CO}_{2}$ es un gas de efecto invernadero (GEI), luego su presencia en la atmósfera influye sobre la temperatura media de la Tierra. Desde la revolución industrial, la concentración de $\mathrm{CO}_{2}$ en la atmósfera se ha venido incrementando continuamente y se tienen evidencias de que eso está causando un cambio climático. Moderar las emisiones de $\mathrm{CO}_{2}$ es, evidentemente, una necesidad a nivel global.

La voluntad de la Unión Europea de reducir las emisiones de GEI se puso de manifiesto al ratificar el protocolo de Kioto en 1998. Los compromisos alcanzados en dicho acuerdo se han articulado en una normativa (Directivas 2003/87/CE y 2009/29/CE) basada, principalmente, en la instauración de un mercado de comercio de derechos de emisión de GEI en el cual las empresas emisoras de GEI pueden vender sus derechos sobrantes a otras empresas con necesidades de emisión.

La llegada del gas natural a la provincia de Castellón a mediados de la década de los 1980 promovió la sustitución de la bicocción tradicional en hornos túnel y de canales, en los que se empleaba fuelóleo o gasóleo como combustible, por la monococción rápida en hornos monoestrato de rodillos, utilizando gas natural (véase §1.4). Este cambio tecnológico supuso, por una parte, una mayor eficiencia energética en la cocción y, por otra, el empleo de un combustible con un menor factor de emisión de $\mathrm{CO}_{2}$. En efecto, las emisiones específicas de $\mathrm{CO}_{2}$ se rebajaron de los $11 \mathrm{~kg} \mathrm{~m}^{-2}$ en 1985 a $\operatorname{los} 5 \mathrm{~kg} \mathrm{~m}^{-2}$ en 1991 [52]; es decir, a la mitad. Sin embargo, desde el inicio de la década de los 1990, este parámetro se ha mantenido más o menos constante [11].

La madurez tecnológica del sector de fabricación de baldosas cerámicas implica que las posibilidades de reducción de sus emisiones de $\mathrm{CO}_{2}$ sean moderadas. Se ha llegado incluso a afirmar que la operación de cocción de baldosas cerámicas ha alcanzado prácticamente la eficiencia máxima [53]. Ante esta perspectiva, una normativa en materia de emisiones de $\mathrm{CO}_{2}$ muy exigente podría promover la deslocalización del sector [54]. En este sentido, el sector de fabricación de baldosas cerámicas figura en el listado de industrias en riesgo de fuga de carbono (Decisión de la Comisión 2014/746/UE), de manera que las empresas tienen asignadas gratuitamente las emisiones de $\mathrm{CO}_{2}$, siempre y cuando éstas no superen las que se establecieron en un benchmarking [55], en cuyo caso tendrían que acudir al mercado de emisiones.

A largo plazo, los objetivos de reducción de emisiones de GEI marcados por la Unión Europea son extremadamente ambiciosos. En la Hoja de Ruta hacia una economía hipocarbónica, publicada por la Comisión Europea, se indican los objetivos de reducción de emisiones para los sectores industriales, entre los que se encuentra el sector cerámico. El objetivo es que, en el año 2050, las emisiones industriales de $\mathrm{CO}_{2}$ se hayan reducido más de un $80 \%$. Este objetivo, aplicado al sector de fabricación de baldosas cerámicas, exige cambios radicales en las tecnologías, de mayor envergadura incluso que los acontecidos en el siglo pasado, como, por ejemplo, sustituir el gas natural por alguna suerte de gas pobre obtenido por fermentación anaerobia de biomasa [11].

La realización de simulaciones por ordenador puede permitir estudiar la viabilidad de cambios revolucionarios en la concepción del horno de rodillos, así como establecer los límites alcanzables con las tecnologías actuales. Por una parte, la simulación es una alternativa a 
la ejecución de experimentos, que podrían requerir un despliegue de recursos prohibitivo, y permite estimar el valor de variables no accesibles experimentalmente. Por otra parte, la simulación es un complemento a la experimentación, ya que ayuda a explicar los resultados experimentales, indagando en las causas, encontrando y clasificando los factores relevantes.

La simulación se ha utilizado satisfactoriamente para el estudio de hornos, calderas y otros equipos de alta temperatura. En la bibliografía se encuentran estudios de hornos rotativos [56-58], hornos de fusión de vidrio [59,60], hornos de craqueo [61-64], hornos de recocido de acero [65-69] o incineradoras [70]. Se han publicado también trabajos de simulación de hornos para la cocción de materiales cerámicos, incluyendo hornos túnel [20,71-73] y hornos de rodillos $[74,75]$. El marco teórico en el que se basan la mayoría de los estudios señalados es el método zonal (\$1.6), posiblemente por su conocida exactitud [76]. De hecho, en la comparación de los distintos métodos de solución de la ecuación de conservación de la energía radiante, el método zonal se utiliza como referencia [37].

Existen dos publicaciones acerca de la simulación de hornos de rodillos para la cocción de baldosas cerámicas [74,75], ambas basadas en el método zonal. El trabajo de Uche y Marín [74] presenta un planteamiento general del problema, identificando los principales términos de los balances. Alves e Sousa y colaboradores [75] se centraron en el caso específico de la puesta en marcha del horno. Pese a que se trata de valiosas contribuciones a la modelización de hornos de rodillos para la cocción de baldosas cerámicas, quedan pendientes por resolver las siguientes necesidades:

- Ofrecer una formulación completa del modelo y de los algoritmos necesarios para resolverlo, de manera que los resultados numéricos puedan ser reproducidos.

- Incorporar en el modelo los últimos avances en materia de transferencia de energía en hornos de rodillos para la cocción de baldosas cerámicas [27-29].

- Someter el modelo a una validación experimental rigurosa.

- Demostrar la capacidad del modelo para realizar predicciones, aplicándolo a una serie de situaciones de interés para el sector de fabricación de baldosas cerámicas.

\subsection{Objetivos}

Para atender a las necesidades identificadas en $§ 2.1$, se plantearon los siguientes objetivos:

- Formular un modelo matemático, basado en el método zonal, que describa la transferencia de energía en los hornos monoestrato de rodillos para la cocción de baldosas cerámicas.

- Definir los algoritmos que permiten resolver el modelo. En particular, el modelo debe permitir calcular la regulación de quemadores con la que se consigue obtener una curva de cocción dada.

- Comprobar la validez del modelo, contrastando valores calculados frente a datos experimentales obtenidos en hornos industriales.

- Aplicar el modelo para valorar la influencia teórica de las acciones recomendadas para el ahorro energético en la cocción de baldosas cerámicas en hornos de rodillos. 


\subsection{Alcance}

El alcance del modelo se limita a la parte de calentamiento del horno, lo que se justifica por los siguientes motivos:

- El calentamiento y el enfriamiento en el horno están bien diferenciados y pueden abordarse por separado [23].

- La combustión del gas natural tiene lugar en el calentamiento. Adoptando unas hipótesis razonables acerca del calor que se recupera del enfriamiento (entalpía del aire de enfriamiento incorporado al calentamiento y temperatura del aire comburente), basta con considerar únicamente el calentamiento para poder predecir el consumo energético del horno.

- A diferencia del enfriamiento, en el calentamiento la transferencia de energía por convección desempeña un papel marginal. Esto permite centrarse en la transferencia por radiación.

- Bajo condiciones apropiadas de funcionamiento del horno, la circulación de los gases en el calentamiento es conocida de antemano, lo que simplifica la formulación del modelo. En el enfriamiento no se da esta situación.

- El calentamiento esta sujeto a un control y monitorización mucho más estrictos, de manera que se tienen datos más accesibles para la validación experimental del modelo.

No obstante, el estudio del enfriamiento tiene un gran interés, tanto desde el punto de vista térmico (recuperación de calor), como de calidad del producto (planaridad, desventados, tensiones residuales, etc. [77]). El modelo podría completarse en un futuro, con la incorporación del enfriamiento.

Por otra parte, el modelo desarrollado ofrece la posibilidad de realizar multitud de simulaciones, pero el conjunto de casos simulados en este trabajo se ha restringido a las medidas convencionales para el ahorro energético. El estudio de tecnologías más innovadoras, como podría ser el uso de sistemas de recuperación de calor, sustitución del gas natural por otros combustibles o modificaciones radicales en la construcción del horno, se plantea como una continuación del presente trabajo. 
We have seen that computer programming is an art, because it applies accumulated knowledge to the world,

because it requires skill and ingenuity, and especially because it produces objects of beauty.

\section{Modelo propuesto}

— Donald E. Knuth [78]

\subsection{Planteamiento general. Hipótesis y simplificaciones}

\subsubsection{Hipótesis generales}

Para expresar la transferencia de energía en el horno de rodillos en términos matemáticos deben realizarse un buen número de suposiciones. Sin ánimo de constituir un listado exhaustivo, a continuación se enumeran ciertas hipótesis generales, lo que permitirá referirse a ellas de manera concisa.

Hipótesis 1. El horno opera en régimen estacionario.

La principal causa de desviaciones con respecto a la hipótesis 1 seguramente sea la falta de continuidad en la producción, en forma de interrupciones ocasionales de la alimentación de baldosas crudas, lo que origina los denominados «huecos» en el argot industrial. Las condiciones de puesta en marcha o parada del horno también son manifiestamente transitorias. Todas estas situaciones quedan fuera del alcance del estudio.

Hipótesis 2. Los gases presentes en el interior del horno se comportan como gases ideales.

Dadas las presiones estáticas que se dan en el interior del horno, las cuales apenas se apartan de la presión atmosférica por unas décimas de milímetro de columna de agua, se considera que la hipótesis 2 está justificada. Las temperaturas relativamente elevadas también contribuirán a la validez de esta hipótesis.

Hipótesis 3. La temperatura de las zonas identificadas en $§ 3.1 .2$ puede considerarse uniforme.

Hipótesis 4. En las zonas de volumen en las que tiene lugar la combustión del gas natural, ésta se produce de manera completa.

La hipótesis 3 es necesaria para la aplicación del método zonal. La hipótesis 4 está relacionada, ya que su validez está condicionada por el tamaño de las zonas de gas (concretamente, por el tiempo de permanencia en las mismas). Ambas hipótesis se discuten detalladamente en $\$ 3.1 .2$.

Hipótesis 5. Las superficies del horno y de las baldosas son superficies grises difusas.

La hipótesis 5 se basa en que la emisividad de los materiales refractarios comúnmente utilizados en los hornos de rodillos es muy alta y apenas varía con la temperatura $[79,80]$. Se asumirá que las baldosas exhiben un comportamiento similar.

Hipótesis 6. El horno es perfectamente estanco.

La hipótesis 6 se refiere a que las únicas entradas y salidas de gases en el horno son las identificadas en la figura 1.8. Existen ciertas características constructivas del horno 
que pueden tener como consecuencia una falta de estanqueidad del mismo. Por ejemplo, para permitir la rotación de los rodillos y facilitar su sustitución, éstos cuentan con una cierta holgura en las paredes del horno, denominada "pasarodillos», que se sella con fibra refractaria. Existe la creencia de que dicha holgura permite la salida de humos en aquellas zonas del horno que se encuentran a una presión estática superior a la atmosférica, así como la admisión de aire ambiente en las zonas en depresión (ver figura 1.8). Asimismo, por la boca del horno también puede acceder gran cantidad de aire al encontrarse próxima al punto de extracción de humos (mínima presión estática) aunque, por este mismo motivo, la región de influencia de dicho aire parásito debería ser limitada.

Hipótesis 7. Las baldosas forman una superficie plana que ocupa toda la anchura útil del horno.

Corolario 7.1 (Definición). A efectos de transferencia radiativa, puede considerarse que el horno está constituido por dos cerramientos: el cerramiento $\mathcal{S}$, por encima de las baldosas, y el cerramiento $\mathcal{I}$, por debajo de las mismas.

Corolario 7.2. Las baldosas suponen una barrera infranqueable para los gases, por lo que no existe mezcla alguna entre los gases que circulan por los cerramientos $\mathcal{S}$ e $\mathcal{I}$.

En la entrada del horno, la hipótesis 7 es una buena aproximación para todos los tipos de productos, puesto que las piezas se alimentan prácticamente de este modo (especialmente los formatos pequeños). No obstante, las composiciones de gres experimentan una contracción significativa durante la cocción, lo que origina que, a medida que las piezas avanzan a lo largo del horno, la ocupación del tapete se reduzca sensiblemente. Aún así, la superficie ocupada sigue siendo muy alta y, además, se modula la velocidad de rotación de los rodillos para prevenir en parte este efecto en el sentido longitudinal.

Como consecuencia de la hipótesis 7, puede postularse que las baldosas (junto con los rodillos) imposibilitan la mezcla de gases entre los cerramientos $\mathcal{S}$ e $\mathcal{I}$ (corolario 7.2). Al parecer, Uche y Marín [74] supusieron que los gases en ambos cerramientos están completamente mezclados. Esto implica que las temperaturas de los gases que circulan por los cerramientos $\mathcal{S}$ e $\mathcal{I}$ tienen que ser iguales en cualquier posición a lo largo del horno, pero prácticamente todos los ciclos de cocción utilizados industrialmente requieren que sean distintas. A igualdad de sencillez, la hipótesis opuesta, adoptada en este trabajo, es susceptible de ofrecer resultados más realistas.

Hipótesis 8. Las paredes «finales» (véase §3.1.2) y los cortafuegos (véase §1.4.1.2) alcanzan prácticamente a las baldosas o a los rodillos, respectivamente, según se encuentren en el canal superior o inferior.

Corolario 8.1. Los cerramientos $\mathcal{S}$ e $\mathcal{I}$ son susceptibles de estar, a su vez, divididos en varios cerramientos en función de la presencia de cortafuegos.

La hipótesis 8 posibilita que el horno pueda considerarse un sistema cerrado en lo que se refiere a la transferencia de radiación térmica. Permitir que escape radiación a través de la boca del horno no plantea excesivas dificultades. Ahora bien, la sección abierta al ambiente es pequeña, especialmente cuando se compara con la superficie total interior del horno. En el caso de los cortafuegos intermedios, que en ocasiones sólo cierran una pequeña porción de la sección de paso, esta hipótesis podría ser más dudosa (ver §4.1).

Hipótesis 9. El horno está regulado de una manera tal que no se incorporan humos a la corriente evacuada por la chimenea de enfriamiento. 
Por último, la hipótesis 9 hace referencia a un adecuado funcionamiento del horno. La incorporación de humos a la zona de enfriamiento puede, por un lado, suponer un aumento del consumo energético $[18,19]$ y, por otro, causar el deterioro de la zona de enfriamiento al no estar preparada para entrar en contacto con los contaminantes presentes en los humos. Por lo tanto, se asumirá que, en este sentido, el horno opera en condiciones apropiadas de funcionamiento.

\subsubsection{División del horno de rodillos en zonas}

Para poder efectuar el análisis del horno en base al método zonal, es necesario dividir el horno en zonas, que pueden ser de superficie o de gas. Estas zonas deben ser lo bastante pequeñas como para poder ser consideradas isotermas (hipótesis 3). Si se cumple esta condición, el método zonal proporciona una solución exacta de la ecuación de la conservación de la energía radiante.

En la práctica, sin embargo, la condición de zonas isotermas para un sistema real sólo se da en el límite en el que éstas son infinitamente pequeñas, pero una división en zonas muy fina podría implicar unos requisitos de tiempo de cálculo y almacenaje de datos prohibitivos. Dependiendo de la aplicación, puede que no se necesite tanto grado de detalle para obtener la información deseada, en tanto en cuanto se tenga la resolución requerida para reproducir la transferencia de energía fielmente $[37,76,81]$.

En el caso concreto del horno de rodillos, su principal característica dimensional es su longitud (en comparación con sus otras dimensiones). En efecto, el ciclo de cocción se especifica estableciendo temperaturas de consigna en posiciones concretas a lo largo del horno que se consideran representativas de toda la sección transversal. En realidad, existen gradientes transversales de temperatura que pueden involucrar diferencias de temperatura de hasta $25^{\circ} \mathrm{C}$ [17]. Si bien dichos gradientes de temperatura son responsables de defectos o falta de consistencia en la producción, cabría esperar que su presencia no tenga una repercusión muy relevante en, por ejemplo, el consumo del horno. En tal caso, su estudio quedaría fuera del alcance del presente trabajo.

En virtud de lo indicado, se optó por dividir el horno en una serie de sectores longitudinales (figura 3.1(a)), los cuales están compuestos por un conjunto de elementos (figura 3.1 (b)) que se corresponden con las zonas del método zonal. Por supuesto, las zonas pertenecientes a un mismo sector pueden encontrarse a una temperatura diferente, pero la temperatura y otras propiedades de cada una de ellas son uniformes. En este sentido, podría decirse que se trata de zonas con parámetros concentrados (no es necesario considerar la distribución espacial de sus parámetros) [82]. La aplicación de este concepto a los volúmenes de gas implica que éstos se encuentran completamente mezclados. Cuando dicha mezcla se extiende al horno en su totalidad, se dice que se trata de un «horno completamente mezclado» (en inglés, well-stirred furnace) [37,44,76,83]. Los modelos de hornos consistentes en una sucesión de volúmenes completamente mezclados se denominan modelos de «horno largo» $[44,76,83]$. Según la terminología de Palmer [84], la división del horno adoptada en este trabajo se trataría de un modelo de horno largo «tipo II», lo que indica que se considera la transferencia de energía radiante entre sectores (en los modelos «tipo I», por el contrario, ésta se desprecia). 


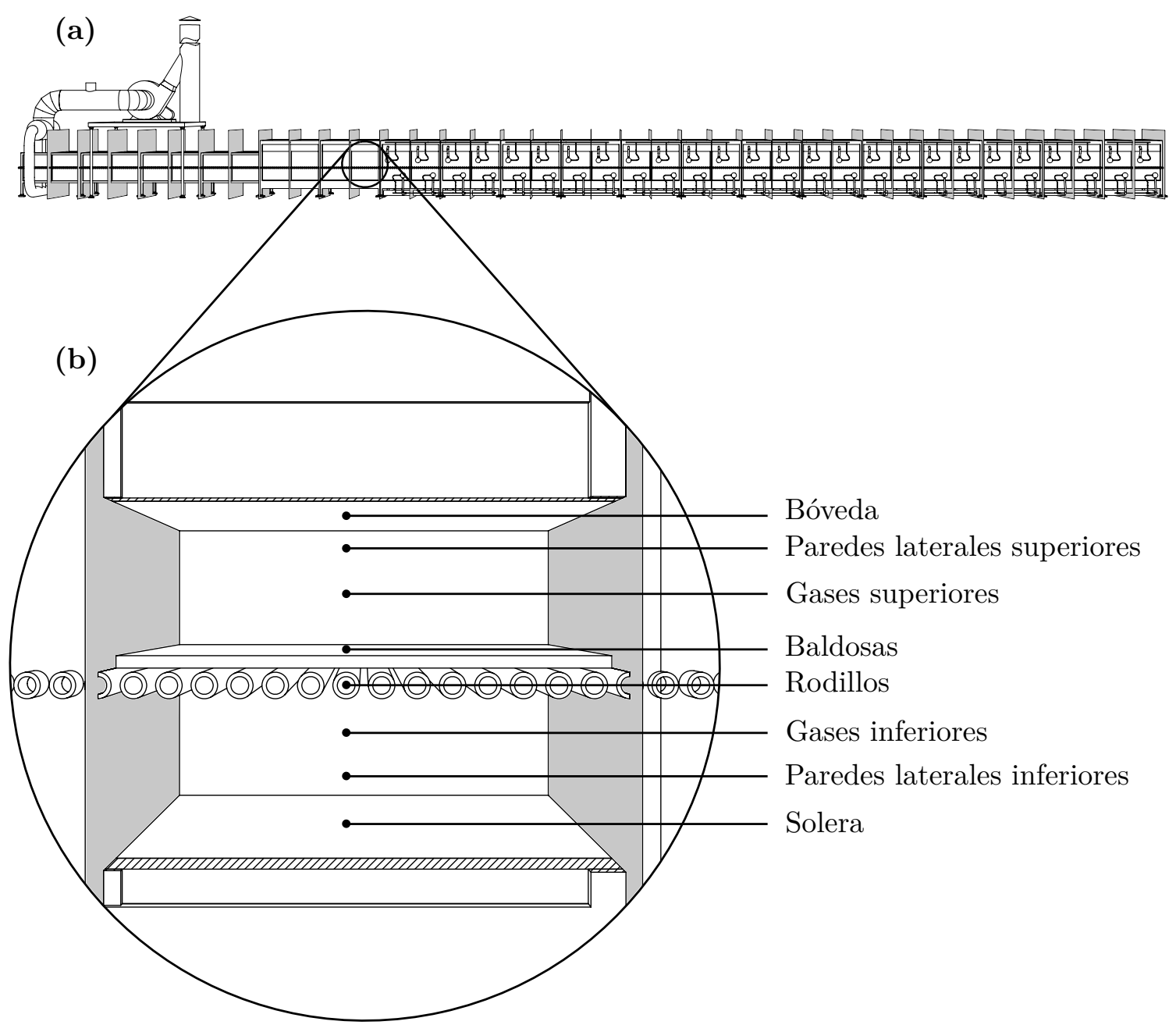

Figura 3.1. (a) División del horno en sectores. (b) Zonas consideradas en cada sector.

Una ventaja de utilizar un modelo de horno largo es que otorga mucha flexibilidad en lo referente a la incorporación (o extracción) de gases a lo largo del horno. Debido a la gran cantidad de quemadores presentes en el horno de rodillos, y a cómo se encuentran éstos distribuidos a lo largo del horno, es imprescindible contar con esta flexibilidad. Al estar limitado el alcance del modelo a la parte de calentamiento del horno (§2.3), puede considerarse que los gases circulan en contracorriente con respecto al sentido de avance de las baldosas. En aquellos sectores en los que existan quemadores, teniendo en cuenta la hipótesis 4, el aire en exceso y los productos de combustión se incorporarán a la corriente gaseosa. Estas consideraciones imponen límites relativos al tamaño de los sectores: las zonas de volumen deben ser lo suficientemente extensas como para que la circulación de gases a través de las zonas se aproxime a la indicada y la hipótesis 4 sea válida.

La división del horno en zonas se completa con las «paredes finales» (por el término end walls utilizado en la literatura en lengua inglesa [85-87]). Las paredes finales son aquellas que se encuentran en el primer y último sector, respectivamente, y que hacen que se trate de un sistema cerrado para la radiación térmica. Así pues, se trataría de la pared a la entrada del horno y del cortafuegos que divide el calentamiento del enfriamiento. 


\subsubsection{Otras consideraciones}

Por ser la práctica habitual en la industria, y por simplificar sustancialmente la formulación de los balances de materia, los volúmenes de gases (en particular, los caudales volumétricos) se expresarán siempre en condiciones normales, haciendo referencia a $0^{\circ} \mathrm{C}$ y $1 \mathrm{~atm}$, convenio al que se adherirá esta memoria.

El estado base termodinámico seleccionado, que siguiendo las recomendaciones de la IUPAC [88] se denotará con el superíndice $\theta$, consiste en las especies en el estado de agregación en el que se encuentran en el horno, sujetas a una presión de $1 \mathrm{~atm}$. La temperatura de referencia $T_{\text {ref }}$ se considera igual a $25^{\circ} \mathrm{C}$, al estar la mayoría de datos termodinámicos tabulados para esta temperatura de referencia y el estado base señalado.

\section{2. Áreas de intercambio directo}

\subsubsection{Tipos de intercambios directos}

Tras dividir el horno en zonas, el método zonal requiere calcular el intercambio directo de energía radiante entre dichas zonas, obteniendo, como resultado, las matrices de DEA: $[\overline{\mathbf{g g}}],[\overline{\mathbf{s s}}]$ y $[\overline{\mathbf{g s}}]$. Puesto que el horno esta compuesto por (al menos) dos cerramientos (corolario 7.1), deben calcularse estas matrices para cada cerramiento.

Al tratarse de un modelo de horno largo tipo II, las zonas pertenecientes a un sector dado pueden intercambiar directamente energía radiante con las zonas de cualquier otro sector, con la única condición de que sean visibles la una por la otra. Las matrices de DEA deben incluir todos los posibles intercambios.

En el caso del intercambio entre gases, sólo existe un tipo, cuyo cálculo viene descrito en $\S 3.2 .2$ y sirve tanto para el cerramiento $\mathcal{S}$ como para el $\mathcal{I}$. Por el principio de reciprocidad (simetría de $[\mathbf{g g}]$ ), es necesario calcular $n \frac{n+1}{2}$ de estos intercambios por cerramiento, donde $n$ es el número de volúmenes de gas en cada cerramiento (que coincide con el número de sectores). Imponiendo ciertas restricciones, podría reducirse el número de DEA entre gases que es necesario calcular a solamente $2 n$, pero, como se señala más adelante, estas restricciones no son aplicables al horno de rodillos.

En el caso de los intercambios entre superficies, se tienen muchos más tipos distintos. Esto es un reflejo de que cada sector cuenta con varias superficies diferentes, con peculiaridades geométricas propias. Se ha considerado conveniente mostrar las posibles combinaciones gráficamente en la figura 3.2. En dicha figura, los tipos de intercambios se han identificado con los siguientes símbolos (se indican entre paréntesis el número de DEA a calcular y la sección en la que se definen):

- intercambio entre superficies planas paralelas $\left(\frac{3}{2} n(n+1), \S 3.2 .3 .1\right)$

$\odot$ intercambio entre superficies planas perpendiculares $(n(n+1)$, §3.2.3.2)

$\diamond$ intercambio entre una superficie plana y una pared final $(4 n, \S 3.2 .3 .3)$

* intercambio entre las paredes finales $(2, \S 3.2 .3 .4)$

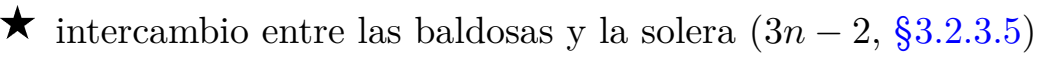


(a)

\begin{tabular}{|c|c|c|c|c|c|}
\hline & 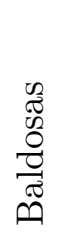 & 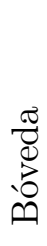 & 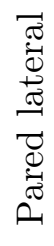 & 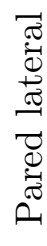 & 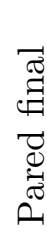 \\
\hline Baldosas & & 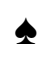 & O & $\varnothing$ & $\diamond$ \\
\hline Bóveda & 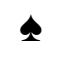 & & Q & $\varnothing$ & $v$ \\
\hline Pared lateral & $\varnothing$ & $\varnothing$ & & s & 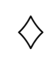 \\
\hline Pared lateral & $\varnothing$ & $\varnothing$ & & & \\
\hline Pared final & $\diamond$ & $\diamond$ & $\Delta$ & $\diamond$ & \\
\hline Pared final & $\diamond$ & $\diamond$ & & $\diamond$ & \\
\hline
\end{tabular}

(b)
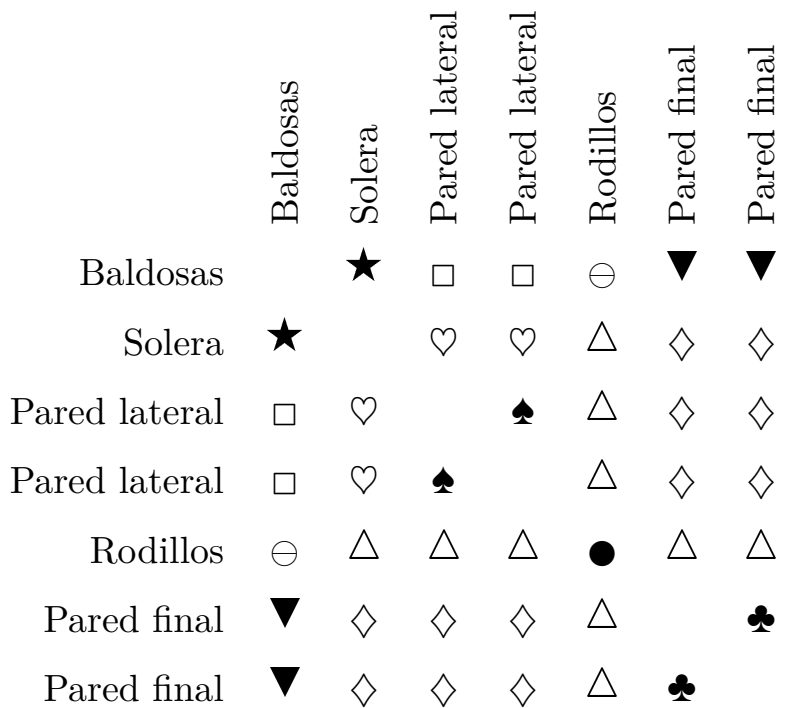

Figura 3.2. Tipos de intercambios directos entre superficies: (a) cerramiento $\mathcal{S}$, (b) cerramiento $\mathcal{I}$.

$\square$ intercambio entre las baldosas y las paredes laterales $(3 n-2, \S 3.2 .3 .6)$

$\boldsymbol{\nabla}$ intercambio entre las baldosas y las paredes finales $(2, \S 3.2 .3 .7)$

$\ominus$ intercambio entre las baldosas y los rodillos $(n, \S 3.2 .3 .8)$

- intercambio entre los rodillos $(n, \S 3.2 .3 .9)$

$\triangle$ intercambio entre los rodillos y las paredes (§3.2.3.10)

Las cantidades de DEA indicadas incluyen ambos cerramientos y se trata de las que es estrictamente necesario calcular, teniendo en cuenta: consideraciones geométricas, el principio de reciprocidad y el principio de Yamauti $[44,45,89,90]$. El caso del intercambio entre los rodillos y las paredes se aborda por un método simplificado y, por este motivo, no se indica el número de DEA a calcular en este caso. 
(a)

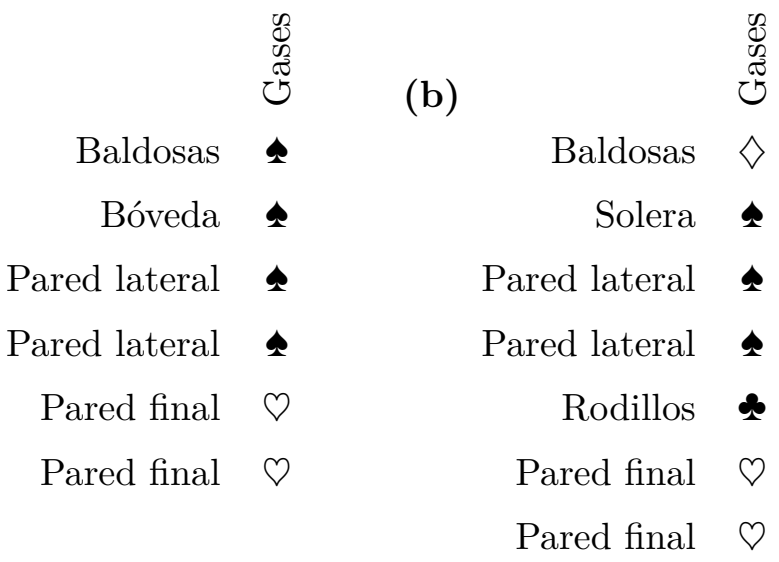

Figura 3.3. Tipos de intercambios directos entre gases y superficies: (a) cerramiento $\mathcal{S}$, (b) cerramiento $\mathcal{I}$.

Los tipos de intercambios entre gases y superficies son menos numerosos que en el caso entre superficies, pero, aún así, contribuyen significativamente al total de DEA a calcular. Los tipos de intercambios entre superficies y gases se identifican en la figura 3.3. Los símbolos que aparecen en dicha figura tienen el siguiente significado (se indica la misma información que para el intercambio entre superficies):

- intercambio entre los gases y las superficies planas $\left(4 n^{2}, \S 3.2 .4 .1\right)$

$\odot$ intercambio entre los gases y las superficies finales $(4 n, \S 3.2 .4 .2)$

$\diamond$ intercambio entre los gases y las baldosas $(3 n-2, \S 3.2 .4 .3)$

* intercambio entre los gases y los rodillos (§3.2.4.4)

En los apartados siguientes, se detallan las expresiones utilizadas para el cálculo de las DEA para todos los tipos de intercambio directo de energía radiante entre las zonas. Los símbolos utilizados para designar a las características geométricas implicadas se definen mediante figuras de apoyo para cada tipo de intercambio. Tras definir todos los tipos de intercambios implicados, se describe el algoritmo de integración utilizado (§3.2.5).

Se permite que, en los intercambios entre sectores diferentes, éstos tengan longitud distinta $\left(L_{1}^{(i)}\right.$ y $\left.L_{1}^{(j)}\right)$. Ello implica renunciar a una propiedad, mencionada anteriormente, de los modelos de horno largo con sectores de longitud constante que evita tener que calcular ciertas DEA - por ejemplo, el intercambio entre los volúmenes de gas en el 1. er y el 3. er sector, respectivamente, sería idéntico al intercambio entre los gases en los sectores $2 .^{\circ}$ y $4 .^{\circ}$, y así sucesivamente- En cualquier caso, puesto que la composición de los gases varía a lo largo del horno, la necesidad de utilizar un coeficiente de absorción medio (véase §A.7) también impide sacar provecho de esta propiedad [91].

Para conseguir que el cálculo de las DEA fuera eficiente, se redujo el orden de integración de las mismas mediante su desarrollo analítico. En dicho desarrollo (que se detalla en §A.4), surgen una serie de funciones «especiales» [92]: integrales exponenciales, funciones gamma incompletas e integrales secantes generalizadas. En $\S$ A.1, se incluye la definición de estas funciones. 
Todos los modelos de WSGG incluyen un gas claro $(K=0)$. Las DEA para gases claros se simplifican considerablemente e incluso, en algunos casos, pueden utilizarse formas cerradas; ambas casuísticas se contemplan en §A.5. En el límite opuesto, en el que el gas puede considerarse opaco, es legítimo utilizar un cálculo simplificado, que se describe en §A.6.

\subsubsection{Intercambio entre volúmenes de gas}

$$
\begin{aligned}
& \overline{g_{i} g_{j}}=I_{1}\left(d-L_{1}^{(i)}, d, L_{1}^{(i)}-d\right)+ \\
&+I_{0}\left(d, d+L_{1}^{(j)}-L_{1}^{(i)}, L_{1}^{(i)}\right)+ \\
&+I_{-1}\left(d-L_{1}^{(j)}-L_{1}^{(i)}, d+L_{1}^{(j)}, d+L_{1}^{(j)}\right) \\
& I_{s}(a, b, c)=\int_{a}^{b} \varphi_{1}(\rho) g(\rho) \rho \mathrm{d} \rho+\int_{b}^{\sqrt{a^{2}+L_{2}^{2}}} \varphi_{2}(\rho) g(\rho) \rho \mathrm{d} \rho+\int_{\sqrt{a^{2}+L_{2}^{2}}}^{\sqrt{b^{2}+L_{2}^{2}}} \varphi_{3}(\rho) g(\rho) \rho \mathrm{d} \rho \\
& \varphi_{1}(\rho)=c L_{2} \operatorname{arc} \cos \frac{a}{\rho}+\frac{s}{2}\left(a^{2}-\rho^{2}\right)+c(a-\rho)+s L_{2} \sqrt{\rho^{2}-a^{2}} \\
& \varphi_{2}(\rho)=c L_{2}\left(\operatorname{arc} \cos \frac{a}{\rho}-\arccos \frac{b}{\rho}\right)+\frac{s}{2}\left(a^{2}-b^{2}\right)+c(a-b)+ \\
&+s L_{2}\left(\sqrt{\rho^{2}-a^{2}}-\sqrt{\rho^{2}-b^{2}}\right) \\
& \varphi_{3}(\rho)=c L_{2}\left(\operatorname{arcsen} \frac{L_{2}}{\rho}-\arccos \frac{b}{\rho}\right)+\frac{s}{2}\left(\rho^{2}+a^{2}-b^{2}\right)-c b+ \\
&+c \sqrt{\rho^{2}-L_{2}^{2}}-s L_{2} \sqrt{\rho^{2}-b^{2}} \\
& g(\rho)= \frac{4 K^{2}}{\pi}\left(\frac{L_{3}}{\rho} \mathrm{S}_{0}\left(K \rho, \operatorname{arctg} \frac{L_{3}}{\rho}\right)-\Gamma_{0}\left(K \rho, K \sqrt{L_{3}^{2}+\rho^{2}}\right)\right)
\end{aligned}
$$

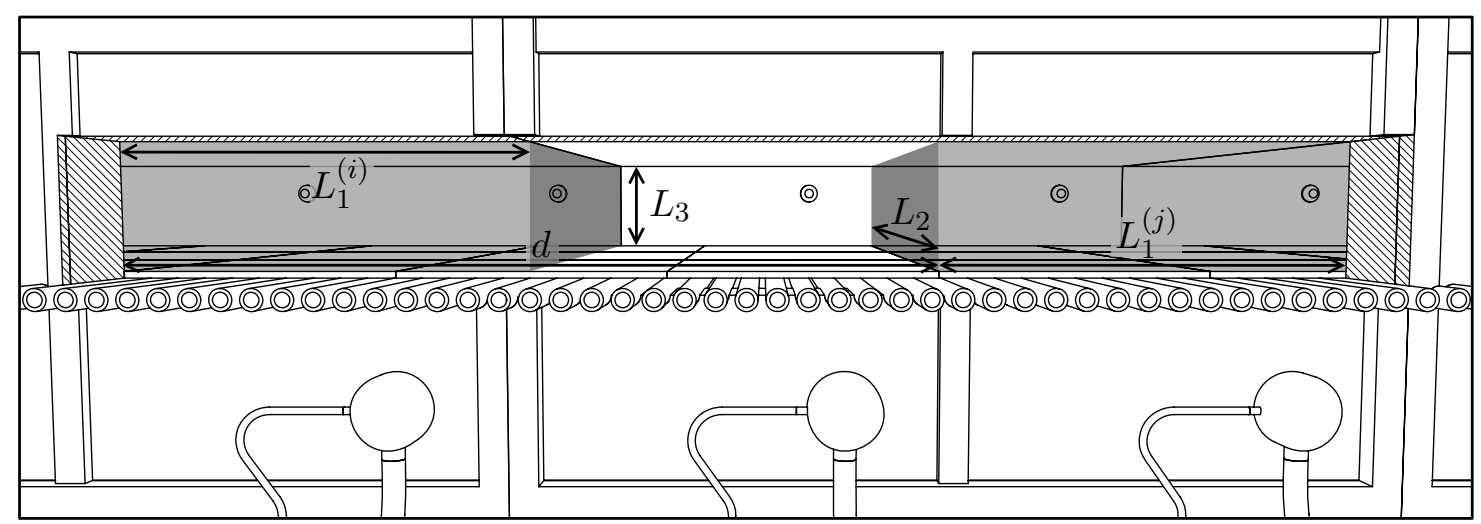

Figura 3.4. Intercambio entre volúmenes de gas. 


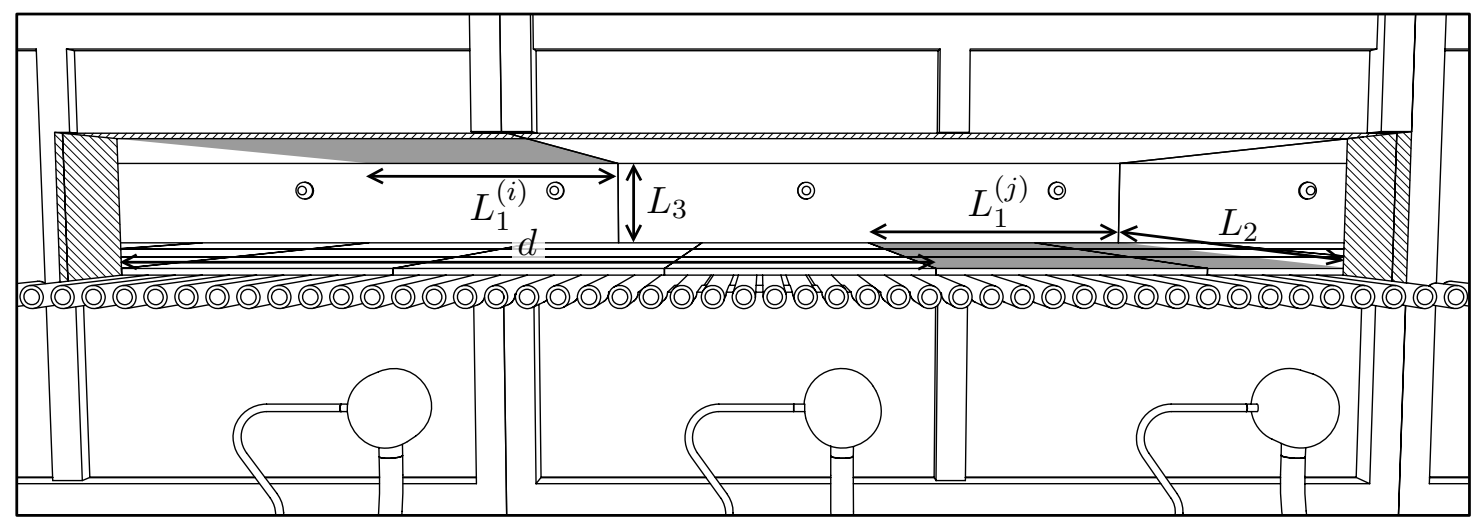

Figura 3.5. Intercambio entre superficies planas paralelas.

\subsubsection{Intercambio entre superficies}

\subsubsection{Intercambio entre superficies planas paralelas}

$$
\begin{aligned}
& \overline{s_{i} s_{j}}=I_{1}\left(d-L_{1}^{(i)}, d, L_{1}^{(i)}-d\right)+ \\
& +I_{0}\left(d, d+L_{1}^{(j)}-L_{1}^{(i)}, L_{1}^{(i)}\right)+ \\
& +I_{-1}\left(d-L_{1}^{(j)}-L_{1}^{(i)}, d+L_{1}^{(j)}, d+L_{1}^{(j)}\right) \\
& I_{S}(a, b, c)=\int_{a}^{b} \varphi_{1}(\rho) g(\rho) \rho \mathrm{d} \rho+\int_{b}^{\sqrt{a^{2}+L_{2}^{2}}} \varphi_{2}(\rho) g(\rho) \rho \mathrm{d} \rho+\int_{\sqrt{a^{2}+L_{2}^{2}}}^{\sqrt{b^{2}+L_{2}^{2}}} \varphi_{3}(\rho) g(\rho) \rho \mathrm{d} \rho \\
& \varphi_{1}(\rho)=c L_{2} \arccos \frac{a}{\rho}+\frac{s}{2}\left(a^{2}-\rho^{2}\right)+c(a-\rho)+s L_{2} \sqrt{\rho^{2}-a^{2}} \\
& \varphi_{2}(\rho)=c L_{2}\left(\arccos \frac{a}{\rho}-\arccos \frac{b}{\rho}\right)+\frac{s}{2}\left(a^{2}-b^{2}\right)+c(a-b)+ \\
& +s L_{2}\left(\sqrt{\rho^{2}-a^{2}}-\sqrt{\rho^{2}-b^{2}}\right) \\
& \varphi_{3}(\rho)=c L_{2}\left(\operatorname{arcsen} \frac{L_{2}}{\rho}-\arccos \frac{b}{\rho}\right)+\frac{s}{2}\left(\rho^{2}+a^{2}-b^{2}\right)-c b+ \\
& +c \sqrt{\rho^{2}-L_{2}^{2}}-s L_{2} \sqrt{\rho^{2}-b^{2}} \\
& g(\rho)=\frac{2 L_{3}^{2}}{\pi} \frac{\mathrm{e}^{-K \sqrt{\rho^{2}+L_{3}^{2}}}}{\left(\rho^{2}+L_{3}^{2}\right)^{2}}
\end{aligned}
$$




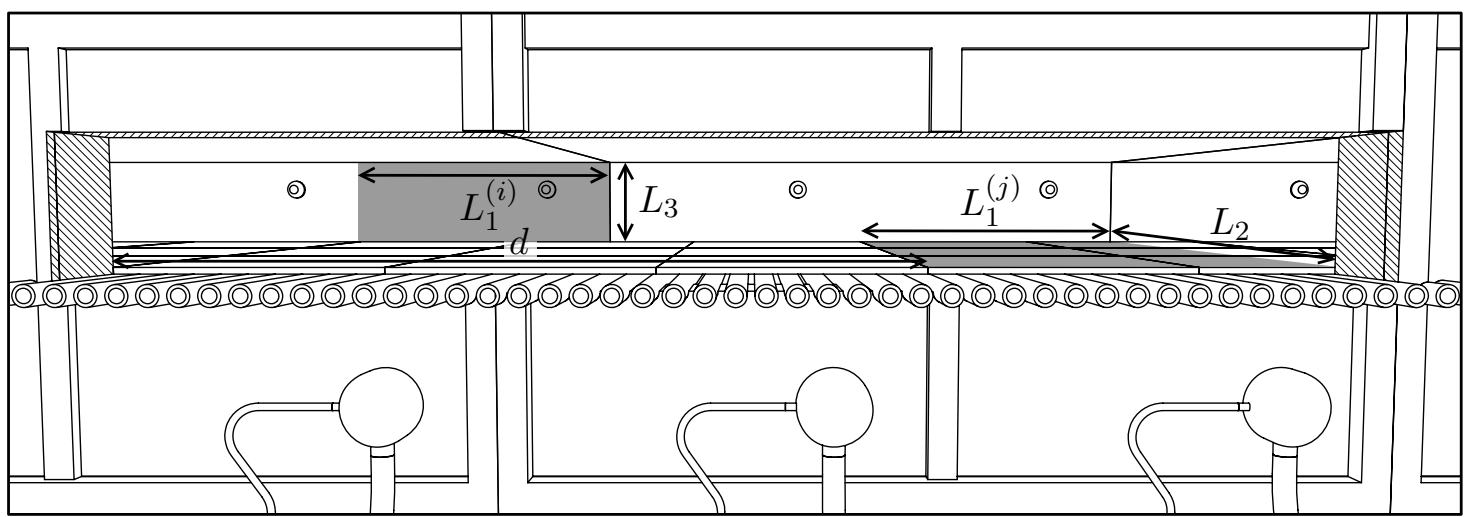

Figura 3.6. Intercambio entre superficies planas perpendiculares.

3.2.3.2. Intercambio entre superficies planas perpendiculares

$$
\begin{aligned}
\overline{s_{i} s_{j}} & =\int_{d-L_{1}^{(i)}}^{d}\left(L_{1}^{(i)}-d+t_{1}\right) f\left(t_{1}\right) \mathrm{d} t_{1}+\int_{d}^{d+L_{1}^{(j)}-L_{1}^{(i)}} L_{1}^{(i)} f\left(t_{1}\right) \mathrm{d} t_{1}+ \\
& +\int_{d+L_{1}^{(j)}-L_{1}^{(i)}}^{d+L_{1}^{(j)}}\left(L_{1}^{(j)}+d-t_{1}\right) f\left(t_{1}\right) \mathrm{d} t_{1} \\
f\left(t_{1}\right) & =\frac{1}{\pi}\left(g\left(\sqrt{t_{1}^{2}+L_{3}^{2}}\right)-g\left(\left|t_{1}\right|\right)+g\left(\sqrt{t_{1}^{2}+L_{2}^{2}}\right)-g\left(\sqrt{t_{1}^{2}+L_{2}^{2}+L_{3}^{2}}\right)\right) \\
g(r) & =\frac{1}{2}\left(\operatorname{Ei}(-K r)+\mathrm{E}_{3}(K r)\right)
\end{aligned}
$$

3.2.3.3. Intercambio entre la superficie de una pared final y una superficie plana

$$
\begin{aligned}
& \overline{s_{i} s_{j}}=\int_{d}^{d+L_{1}} \varphi_{1}(\rho) g(\rho) \rho \mathrm{d} \rho+\int_{d+L_{1}}^{\sqrt{d^{2}+L_{2}^{2}}} \varphi_{2}(\rho) g(\rho) \rho \mathrm{d} \rho+\int_{\sqrt{d^{2}+L_{2}^{2}}}^{\sqrt{\left(d+L_{1}\right)^{2}+L_{2}^{2}}} \varphi_{3}(\rho) g(\rho) \rho \mathrm{d} \rho \\
& \varphi_{1}(\rho)=\frac{1}{2}\left(d^{2}-\rho^{2}\right)+L_{2} \sqrt{\rho^{2}-d^{2}} \\
& \varphi_{2}(\rho)=\frac{1}{2}\left(d^{2}-\left(d+L_{1}\right)^{2}\right)+L_{2}\left(\sqrt{\rho^{2}-d^{2}}-\sqrt{\rho^{2}-\left(d+L_{1}\right)^{2}}\right) \\
& \varphi_{3}(\rho)=\frac{1}{2}\left(\rho^{2}+L_{2}^{2}-\left(d+L_{1}\right)^{2}\right)-L_{2} \sqrt{\rho^{2}-\left(d+L_{1}\right)^{2}} \\
& g(\rho)=\frac{2 K^{2}}{\pi} \Gamma_{-2}\left(K \rho, K \sqrt{\rho^{2}+L_{3}^{2}}\right)
\end{aligned}
$$




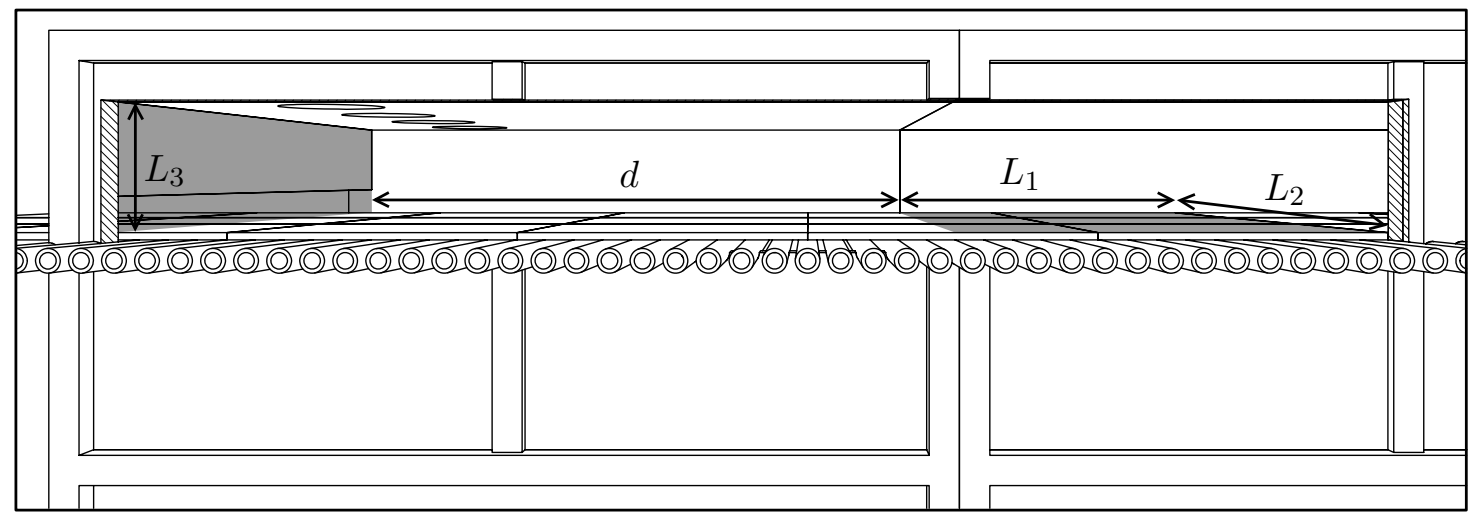

Figura 3.7. Intercambio entre la superficie de una pared final del horno y una superficie plana.

3.2.3.4. Intercambio entre las superficies de las paredes finales

$$
\begin{aligned}
\overline{s_{i} s_{j}} & =\int_{0}^{L_{2}} \varphi_{1}(\rho) g(\rho) \rho \mathrm{d} \rho+\int_{L_{2}}^{L_{3}} \varphi_{2}(\rho) g(\rho) \rho \mathrm{d} \rho+\int_{L_{3}}^{\sqrt{L_{2}^{2}+L_{3}^{2}}} \varphi_{3}(\rho) g(\rho) \rho \mathrm{d} \rho \\
\varphi_{1}(\rho) & =\frac{\pi}{2} L_{2} L_{3}+\frac{\rho^{2}}{2}-\rho\left(L_{2}-L_{3}\right) \\
\varphi_{2}(\rho) & =L_{2} L_{3} \operatorname{arcsen} \frac{L_{2}}{\rho}-\frac{L_{2}^{2}}{2}-\rho L_{3}+L_{3} \sqrt{\rho^{2}-L_{2}^{2}} \\
\varphi_{3}(\rho) & =L_{2} L_{3}\left(\operatorname{arcsen} \frac{L_{2}}{\rho}-\arccos \frac{b}{\rho}\right)-\frac{1}{2}\left(\rho^{2}+L_{2}^{2}+L_{3}^{2}\right)+ \\
& +L_{2} \sqrt{\rho^{2}-L_{3}^{2}}+L_{3} \sqrt{\rho^{2}-L_{2}^{2}} \\
g(\rho)= & \frac{4 d^{2}}{\pi} \frac{\mathrm{e}^{-K \sqrt{d^{2}+\rho^{2}}}}{\left(d^{2}+\rho^{2}\right)^{2}}
\end{aligned}
$$

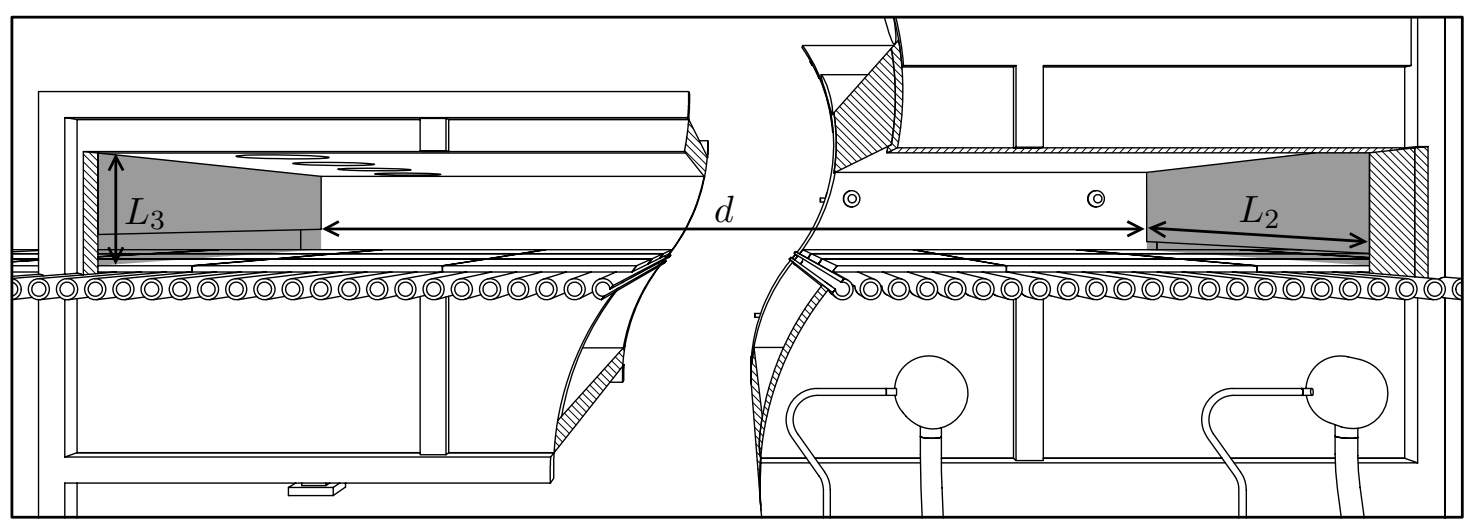

Figura 3.8. Intercambio entre las superficies de las paredes finales. 


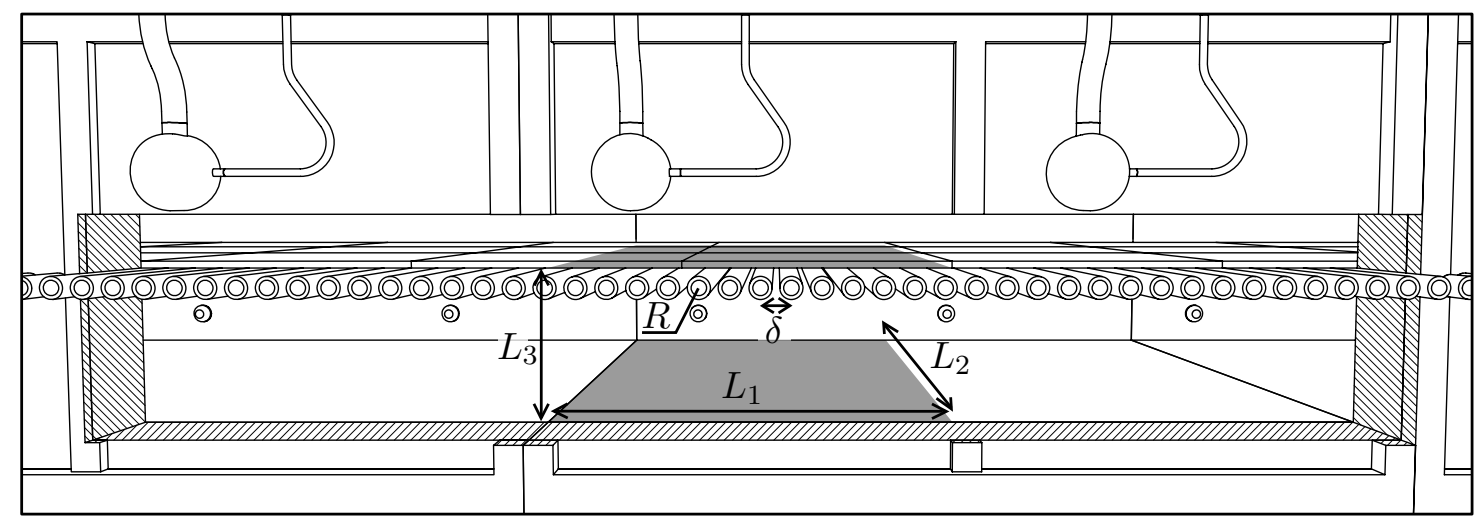

Figura 3.9. Intercambio entre la superficie de la solera y la superficie de las baldosas en contacto con los rodillos.

3.2.3.5. Intercambio entre la superficie de la solera y la superficie de las baldosas en contacto con los rodillos

$$
\begin{aligned}
\overline{s_{i} s_{j}} & = \begin{cases}2\left(\frac{L_{1}}{\delta}-\left\lfloor\frac{x_{i}^{+}}{\delta}+\frac{1}{2}\right\rfloor\right) I_{\infty}+2 \sum_{k=1}^{\frac{x_{i}^{+}}{\delta}+\frac{1}{2}} I_{k} & \text { si están en el mismo sector } \\
\left.\frac{x_{i}^{+}}{\delta}+\frac{1}{2}\right\rfloor I_{\infty}-\sum_{k=1}^{\frac{x_{i}^{+}}{\delta}+\frac{1}{2}} I_{k} & \text { si están en sectores contiguos } \\
0 & \text { en caso contrario }\end{cases} \\
x_{i}^{+}= & \left(L_{3}-R\right) \sqrt{\left(\frac{\delta}{2 R}\right)^{2}-1} \\
I_{\infty}= & \int_{t_{1}^{+}\left(x_{i}^{+}\right)}^{t_{(}^{+}\left(x_{i}^{+}\right)}\left(L_{3}\left(x_{i}^{+}+\frac{\delta}{2}\right)+\left(L_{3}-R\right) t_{1}-R \rho\right) g(\rho) \mathrm{d} t_{1}+ \\
& +\int_{t_{1}^{-}(0)}^{t_{1}^{+}\left(x_{i}^{+}\right)}\left(L_{3} \delta-2 R \rho\right) g(\rho) \mathrm{d} t_{1}+\int_{t_{1}^{-}(0)}^{t_{1}^{+}(0)}\left(L_{3} \frac{\delta}{2}-\left(L_{3}-R\right) t_{1}-R \rho\right) g(\rho) \mathrm{d} t_{1} \\
& \int_{t_{1}^{+}\left(\left(k-\frac{1}{2}\right) \delta\right)}^{t_{1}^{+}\left(\left(k-\frac{1}{2}\right) \delta\right)}\left(\int_{t_{1}^{-}\left(\left(k-\frac{1}{2}\right) \delta\right)}^{t_{1}^{-}(0)}\left(k L_{3} \delta+\left(L_{3}-R\right) t_{1}-R \rho\right) g(\rho) \mathrm{d} t_{1}+\right.
\end{aligned}
$$


$\rho=\rho\left(t_{1}\right)=\sqrt{t_{1}^{2}+L_{3}^{2}}$

$$
\begin{aligned}
& g(\rho)=\frac{L_{2}^{2} L_{3}}{\pi \rho^{2}}\left(\frac{\mathrm{e}^{-K \sqrt{t_{2}^{2}+\rho^{2}}}}{L_{2}^{2}+\rho^{2}}-K \frac{\mathrm{e}^{-K \sqrt{t_{2}^{2}+\rho^{2}}}}{\sqrt{t_{2}^{2}+\rho^{2}}}\right)+ \\
& +\frac{L_{2} L_{3}}{\pi \rho^{3}}\left(\left(1+K^{2} \rho^{2}\right) \mathrm{S}_{0}\left(K \rho, \operatorname{arctg} \frac{L_{2}}{\rho}\right)+\mathrm{S}_{1}\left(K \rho, \operatorname{arctg} \frac{L_{2}}{\rho}\right)-\right. \\
& \left.-\mathrm{S}_{2}\left(K \rho, \operatorname{arctg} \frac{L_{2}}{\rho}\right)\right)-\frac{2 L_{3} K^{2}}{\pi} \Gamma_{-2}\left(K \rho, K \sqrt{L_{2}^{2}+\rho^{2}}\right) \\
& t_{1}^{-}\left(x_{i}\right)=-\left(L_{3}-R\right) \frac{x_{i}+\frac{\delta}{2}-R \sqrt{1-\frac{R^{2}-\left(x_{i}+\frac{\delta}{2}\right)^{2}}{\left(L_{3}-R\right)^{2}}}}{L_{3}-2 R} \\
& t_{1}^{+}\left(x_{i}\right)=-\left(L_{3}-R\right) \frac{x_{i}-\frac{\delta}{2}+R \sqrt{1-\frac{R^{2}-\left(x_{i}-\frac{\delta}{2}\right)^{2}}{\left(L_{3}-R\right)^{2}}}}{L_{3}-2 R}
\end{aligned}
$$

3.2.3.6. Intercambio entre la superficie de las paredes laterales y la superficie de las baldosas en contacto con los rodillos

$$
\overline{s_{i} s_{j}}= \begin{cases}2\left(\frac{L_{1}}{\delta}-\left\lfloor\frac{x_{i}^{+}}{\delta}+\frac{1}{2}\right\rfloor\right) I_{\infty}+2 \sum_{k=1}^{\frac{x_{i}^{+}}{\delta}+\frac{1}{2}} I_{k} & \text { si están en el mismo sector } \\ \left\lfloor\frac{x_{i}^{+}}{\delta}+\frac{1}{2}\right\rfloor I_{\infty}-\sum_{k=1}^{\frac{x_{i}^{+}}{\delta}+\frac{1}{2}} I_{k} & \text { si están en sectores contiguos } \\ 0 & \text { en caso contrario }\end{cases}
$$

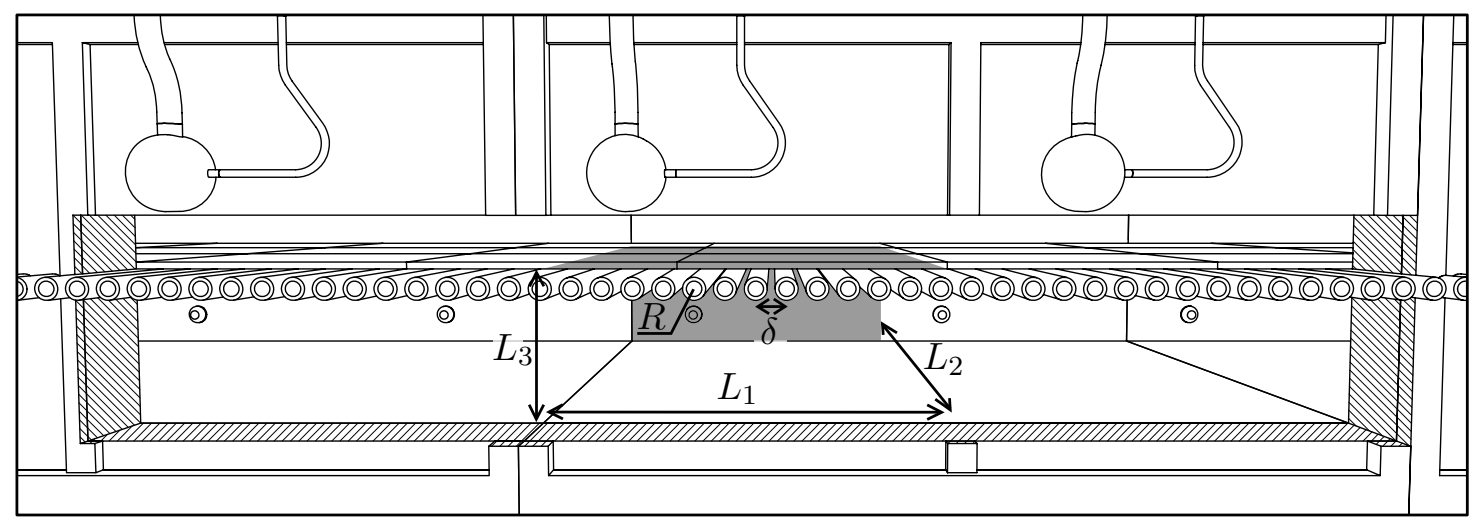

Figura 3.10. Intercambio entre la superficie de las paredes laterales y la superficie de las baldosas en contacto con los rodillos. 


$$
\begin{aligned}
x_{i}^{+} & =\left(L_{3}-R\right) \sqrt{\left(\frac{\delta}{2 R}\right)^{2}-1} \\
I_{\infty} & =\int_{0}^{L_{3}-R} \int_{t_{1}^{+}\left(x_{i}^{+}, z_{i}\right)}^{t_{1}^{-}\left(x_{i}^{+}, z_{i}\right)}\left(\left(L_{3}-z_{i}\right)\left(x_{i}^{+}+\frac{\delta}{2}\right)+\left(L_{3}-R-z_{i}\right) t_{1}-R \rho\right) g(\rho) \mathrm{d} t_{1} \mathrm{~d} z_{i}+ \\
& +\int_{0}^{L_{3}-R} \int_{1}^{t_{1}^{-}\left(0, z_{i}\right)}\left(\left(L_{3}-z_{i}\right) \delta-2 R \rho\right) g(\rho) \mathrm{d} t_{1} \mathrm{~d} z_{i}+ \\
& +\int_{0}^{L_{3}-R} \int_{t_{1}^{+}\left(0, z_{i}\right)}^{t_{1}^{-}\left(0, z_{i}\right)}\left(\left(L_{3}-z_{i}\right) \frac{\delta}{2}-\left(L_{3}-R-z_{i}\right) t_{1}-R \rho\right) g(\rho) \mathrm{d} t_{1} \mathrm{~d} z_{i}
\end{aligned}
$$

$$
\begin{aligned}
I_{k} & =\int_{0}^{L_{3}-R} \int_{t_{1}^{-}\left(\left(k-\frac{1}{2}\right) \delta, z_{i}\right)}^{t_{1}^{+}\left(\left(k-\frac{1}{2}\right) \delta, z_{i}\right)}\left(k\left(L_{3}-z_{i}\right) \delta+\left(L_{3}-R-z_{i}\right) t_{1}-R \rho\right) g(\rho) \mathrm{d} t_{1} \mathrm{~d} z_{i}+ \\
& +\int_{0}^{L_{3}-R} \int_{t_{1}^{+}\left(\left(k-\frac{1}{2}\right) \delta, z_{i}\right)}^{t_{1}^{-}\left(0, z_{i}\right)}\left(\left(L_{3}-z_{i}\right) \delta-2 R \rho\right) g(\rho) \mathrm{d} t_{1} \mathrm{~d} z_{i}+ \\
& +\int_{0}^{L_{3}-R} \int_{t_{1}^{-}\left(0, z_{i}\right)}^{t_{1}^{+}\left(0, z_{i}\right)}\left(\left(L_{3}-z_{i}\right) \frac{\delta}{2}-\left(L_{3}-R-z_{i}\right) t_{1}-R \rho\right) g(\rho) \mathrm{d} t_{1} \mathrm{~d} z_{i}
\end{aligned}
$$$$
\rho=\rho\left(t_{1}, z_{i}\right)=\sqrt{t_{1}^{2}+\left(L_{3}-z_{i}\right)^{2}}
$$

$g(\rho)=\frac{K^{2}}{\pi} \Gamma_{-2}\left(K \rho, K \sqrt{L_{2}+\rho^{2}}\right)$

$$
\begin{aligned}
t_{1}^{-}\left(x_{i}, z_{i}\right) & =-\left(L_{3}-R-z_{i}\right) \frac{x_{i}+\frac{\delta}{2}-R \sqrt{1-\frac{R^{2}-\left(x_{i}+\frac{\delta}{2}\right)^{2}}{\left(L_{3}-R-z_{i}\right)^{2}}}}{L_{3}-2 R-z_{i}} \\
t_{1}^{+}\left(x_{i}, z_{i}\right) & =-\left(L_{3}-R-z_{i}\right) \frac{x_{i}-\frac{\delta}{2}+R \sqrt{1-\frac{R^{2}-\left(x_{i}-\frac{\delta}{2}\right)^{2}}{\left(L_{3}-R-z_{i}\right)^{2}}}}{L_{3}-2 R-z_{i}}
\end{aligned}
$$




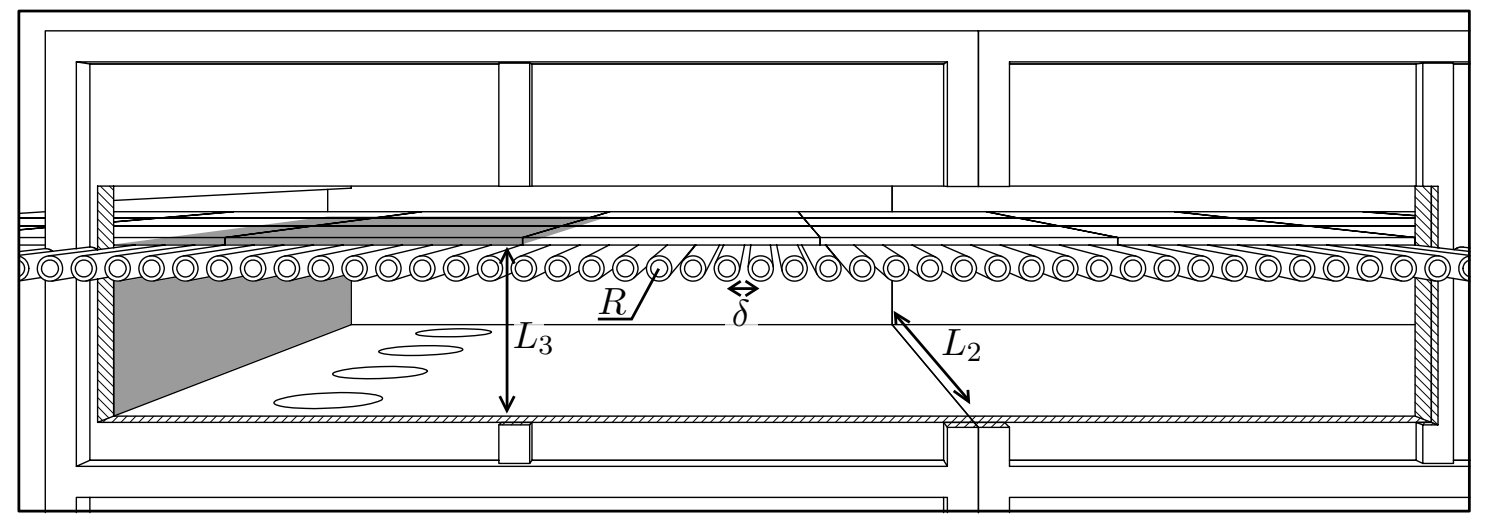

Figura 3.11. Intercambio entre la superficie de una pared final y la superficie de las baldosas en contacto con los rodillos.

3.2.3.7. Intercambio entre la superficie de una pared final y la superficie de las baldosas en contacto con los rodillos

$$
\begin{aligned}
& \overline{s_{i} s_{j}}= \begin{cases}\sum_{k=1}^{\frac{x_{i}^{+}}{\delta}+\frac{1}{2}} I_{k} & \text { si están en el mismo sector } \\
0 & \text { en caso contrario }\end{cases} \\
& I_{k}=2 \int_{0}^{z_{i}^{+}} \int_{0}^{L_{2}}\left(L_{2}-t_{2}\right)\left(L_{3}-z_{i}\right) g\left(\rho, z_{i}\right) \mathrm{d} t_{2} \mathrm{~d} z_{i}
\end{aligned}
$$




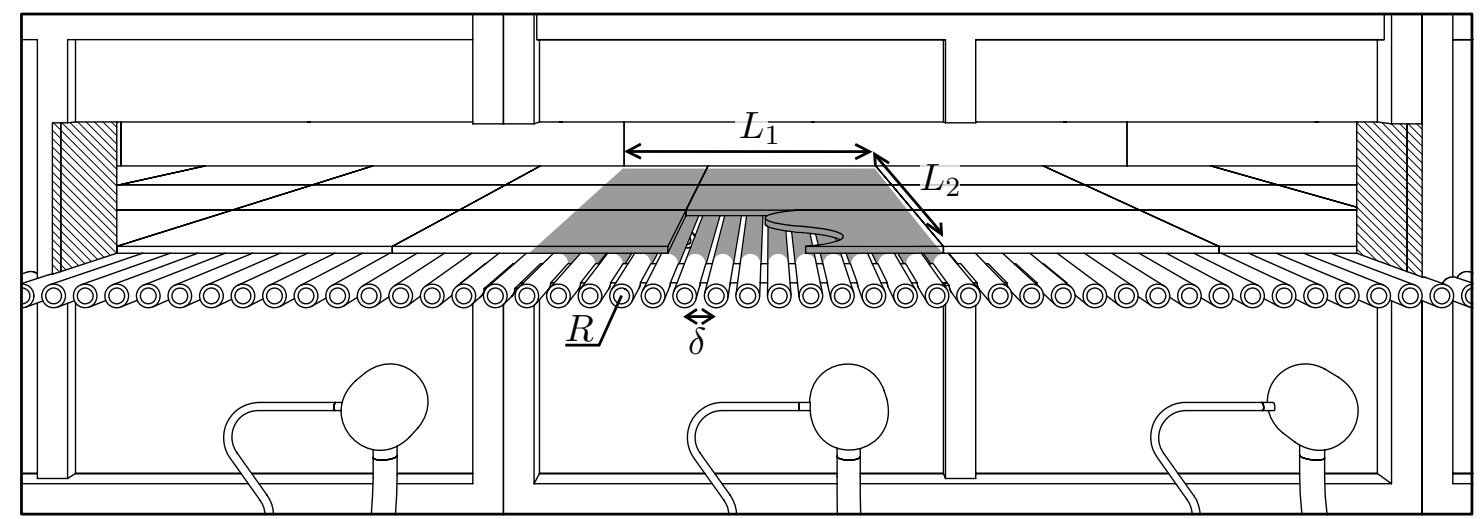

Figura 3.12. Intercambio entre la superficie de las baldosas en contacto con los rodillos y la superficie de los rodillos.

3.2.3.8. Intercambio entre la superficie de las baldosas en contacto con los rodillos y la superficie de los rodillos

$$
\begin{aligned}
& \overline{s_{i} s_{j}}=\frac{L_{1} L_{2} R}{\pi \delta} \int_{0}^{\delta} \int_{\alpha_{j}^{-}\left(x_{i}\right)}^{\frac{3 \pi}{2}} f\left(x_{i}, \alpha_{j}\right) g(\rho) \mathrm{d} \alpha_{j} \mathrm{~d} x_{i} \\
& f\left(x_{i}, \alpha_{j}\right)=R\left(1+\operatorname{sen} \alpha_{j}\right)\left(\left(x_{i}-\delta\right) \cos \alpha_{j}-R\left(1+\operatorname{sen} \alpha_{j}\right)\right) \\
& g(\rho)=\frac{1}{\rho^{3}}\left(\left(1+K^{2} \rho^{2}\right) \mathrm{S}_{0}\left(K \rho, \frac{\pi}{2}\right)+\mathrm{S}_{1}\left(K \rho, \frac{\pi}{2}\right)-\mathrm{S}_{2}\left(K \rho, \frac{\pi}{2}\right)\right) \\
& \rho=\rho\left(x_{i}, \alpha_{j}\right)=\sqrt{\left(\delta+R \cos \alpha_{j}-x_{i}\right)^{2}+R^{2}\left(1+\operatorname{sen} \alpha_{j}\right)^{2}} \\
& \alpha_{j}^{-}\left(x_{i}\right)=\left\{\begin{array}{l}
\text { si } x_{i} \geq \frac{\delta}{2}-\frac{\delta}{2} \sqrt{1-\left(\frac{2 R}{\delta}\right)^{2}} \\
2 \operatorname{arctg} \frac{x_{i}^{2}-R^{2}-2 \sqrt{\delta x_{i}\left(R^{2}+x_{i}\left(x_{i}-\delta\right)\right)}}{\left(x_{i}+R\right)^{2}-2 \delta x_{i}}
\end{array}\right. \\
& \operatorname{arctg} \frac{\left(x_{i}-\delta\right)^{2}-R^{2}}{2 R\left(x_{i}-\delta\right)}
\end{aligned}
$$

\subsubsection{Intercambio entre las superficies de los rodillos}

$$
\overline{s_{i} s_{j}}=\frac{4 L_{1} L_{2} R^{2}}{\pi \delta} \int_{0}^{\frac{\pi}{2}} \int_{\alpha_{j}^{-}\left(\alpha_{i}\right)}^{\alpha_{j}^{+}\left(\alpha_{i}\right)} f\left(\alpha_{i}, \alpha_{j}\right) g(\rho) \mathrm{d} \alpha_{j} \mathrm{~d} \alpha_{i}
$$




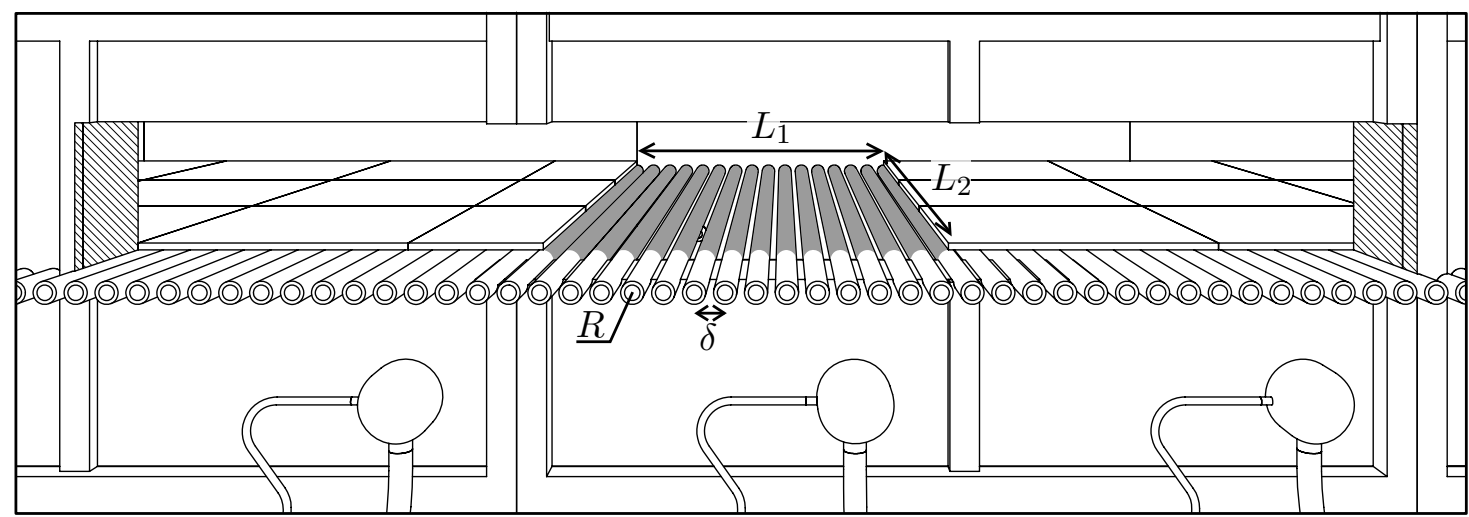

Figura 3.13. Intercambio entre las superficies de los rodillos.

$$
f\left(\alpha_{i}, \alpha_{j}\right)=\left(R\left(\cos \left(\alpha_{j}-\alpha_{i}\right)-1\right)+\delta \cos \alpha_{i}\right)\left(R\left(\cos \left(\alpha_{j}-\alpha_{i}\right)-1\right)+\delta \cos \alpha_{j}\right)
$$

$$
\begin{aligned}
& g(\rho)=\frac{1}{\rho^{3}}\left(\left(1+K^{2} \rho^{2}\right) \mathrm{S}_{0}\left(K \rho, \frac{\pi}{2}\right)+\mathrm{S}_{1}\left(K \rho, \frac{\pi}{2}\right)-\mathrm{S}_{2}\left(K \rho, \frac{\pi}{2}\right)\right) \\
& \rho=\rho\left(\alpha_{i}, \alpha_{j}\right)=\sqrt{\left(\delta+R\left(\cos \alpha_{j}-\cos \alpha_{i}\right)\right)^{2}+R^{2}\left(\operatorname{sen} \alpha_{j}-\operatorname{sen} \alpha_{i}\right)^{2}} \\
& \alpha_{j}^{-}\left(\alpha_{i}\right)=2 \operatorname{arctg} \frac{R \operatorname{sen} \alpha_{i}-\sqrt{\delta^{2}-2 R \delta \cos \alpha_{i}}}{R\left(1+\cos \alpha_{i}\right)-\delta} \\
& \alpha_{j}^{+}\left(\alpha_{i}\right)= \begin{cases}2 \operatorname{arctg} \frac{R \operatorname{sen} \alpha_{i}+\sqrt{\delta^{2}-2 R \delta \cos \alpha_{i}}}{R\left(1+\cos \alpha_{i}\right)-\delta} & \text { si } \alpha_{i} \leq \arccos \frac{2 R}{\delta} \\
2 \operatorname{arctg} \frac{R \operatorname{sen} \alpha_{i}+\sqrt{2 R \delta \cos \alpha_{i}-\delta^{2} \cos ^{2} \alpha_{i}}}{R\left(1+\cos \alpha_{i}\right)-\delta \cos \alpha_{i}} & \text { si } \alpha_{i}>\arccos \frac{2 R}{\delta}\end{cases}
\end{aligned}
$$

\subsubsection{Intercambio entre la superficie de los rodillos y las superficies de las paredes}

La evaluación de las DEA para estos tipos de intercambios a partir de expresiones matemáticas explícitas sería extremadamente ardua. Cada rodillo tiene, dependiendo de su posición en el sector del que forma parte, una manera propia de intercambiar directamente radiación térmica con las paredes. En otras palabras, debería calcularse el DEA entre (prácticamente) cada rodillo y cada pared, lo que involucraría la evaluación de una cantidad enorme de integrales que apenas puede reducirse apelando a la simetría.

Para evitar introducir este cuello de botella en los cálculos, se utilizó un procedimiento simplificado inspirado en el ejemplo 5 de la referencia [79]. En este procedimiento, se calcula, en primer lugar, el intercambio entre la superficie de la pared en cuestión y una superficie plana imaginaria que contiene los ejes de los rodillos (figura 3.14), por medio de 


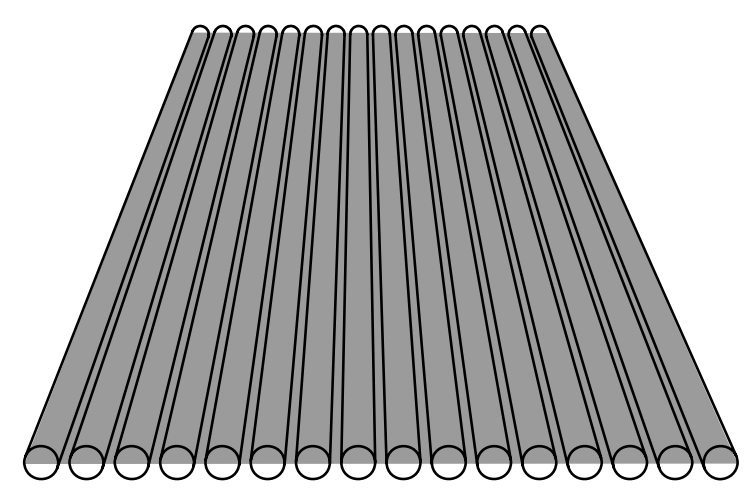

Figura 3.14. Plano que contiene los ejes de los rodillos.

las expresiones recogidas en $\S 3.2 .3 .1$, §3.2.3.2 o $\$ 3.2 .3 .3$, según el caso. A este valor se le sustrae el DEA entre la pared y las baldosas $(\S 3.2 .3 .5, \S 3.2 .3 .6$ o $\S 3.2 .3 .7)$, para tener en cuenta la radiación no interceptada por los rodillos. El resultado es una aproximación del DEA entre los rodillos y la pared.

Para gases claros este procedimiento es exacto, pero en presencia de gases grises se comete un cierto error, que debe corregirse posteriormente (§3.2.6), al no evaluar correctamente la absorción de radiación por parte del gas en el entorno inmediato de los rodillos. En un trabajo reciente [68], se ha utilizado una estrategia similar para calcular las DEA para lingotes de acero en un horno de recocido de solera galopante.

\subsubsection{Intercambio entre volúmenes de gas y superficies}

\subsubsection{Intercambio entre un volumen de gas y una superficie plana}

$$
\begin{aligned}
\overline{g_{i} s_{j}} & =I_{1}\left(d-L_{1}^{(i)}, d, L_{1}^{(i)}-d\right)+ \\
& +I_{0}\left(d, d+L_{1}^{(j)}-L_{1}^{(i)}, L_{1}^{(i)}\right)+ \\
& +I_{-1}\left(d-L_{1}^{(j)}-L_{1}^{(i)}, d+L_{1}^{(j)}, d+L_{1}^{(j)}\right)
\end{aligned}
$$

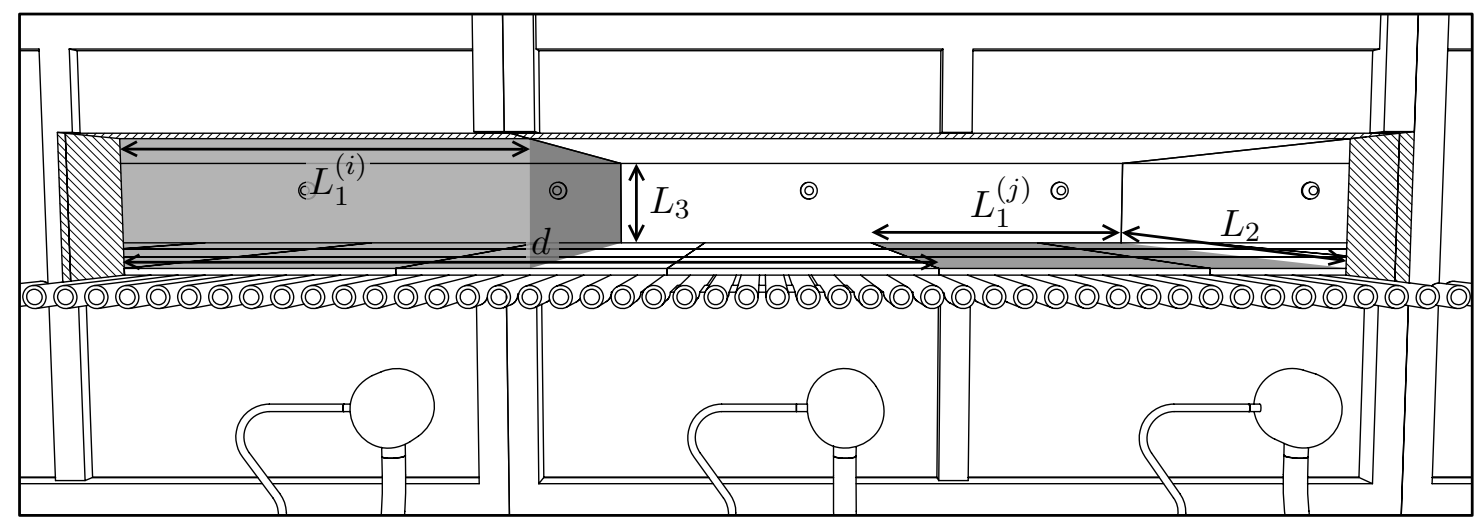

Figura 3.15. Intercambio entre un volumen de gas y una superficie plana. 


$$
\begin{aligned}
& I_{s}(a, b, c)=\int_{a}^{b} \varphi_{1}(\rho) g(\rho) \rho \mathrm{d} \rho+\int_{b}^{\sqrt{a^{2}+L_{2}^{2}}} \varphi_{2}(\rho) g(\rho) \rho \mathrm{d} \rho+\int_{\sqrt{a^{2}+L_{2}^{2}}}^{\sqrt{b^{2}+L_{2}^{2}}} \varphi_{3}(\rho) g(\rho) \rho \mathrm{d} \rho \\
& \varphi_{1}(\rho)=c L_{2} \arccos \frac{a}{\rho}+\frac{s}{2}\left(a^{2}-\rho^{2}\right)+c(a-\rho)+s L_{2} \sqrt{\rho^{2}-a^{2}} \\
& \varphi_{2}(\rho)=c L_{2}\left(\arccos \frac{a}{\rho}-\arccos \frac{b}{\rho}\right)+\frac{s}{2}\left(a^{2}-b^{2}\right)+c(a-b)+ \\
& +s L_{2}\left(\sqrt{\rho^{2}-a^{2}}-\sqrt{\rho^{2}-b^{2}}\right) \\
& \varphi_{3}(\rho)=c L_{2}\left(\operatorname{arcsen} \frac{L_{2}}{\rho}-\arccos \frac{b}{\rho}\right)+\frac{s}{2}\left(\rho^{2}+a^{2}-b^{2}\right)-c b+ \\
& +c \sqrt{\rho^{2}-L_{2}^{2}}-s L_{2} \sqrt{\rho^{2}-b^{2}} \\
& g(\rho)=\frac{2 K^{2}}{\pi} \Gamma_{-1}\left(K \rho, K \sqrt{\rho^{2}+L_{3}^{2}}\right)
\end{aligned}
$$

3.2.4.2. Intercambio entre un volumen de gas y la superficie de una pared final

$$
\begin{aligned}
& \overline{g_{i} s_{j}}=\int_{d}^{d+L_{1}} \varphi_{1}(\rho) g(\rho) \rho \mathrm{d} \rho+\int_{d+L_{1}}^{\sqrt{d^{2}+L_{2}^{2}}} \varphi_{2}(\rho) g(\rho) \rho \mathrm{d} \rho+\int_{\sqrt{d^{2}+L_{2}^{2}}}^{\sqrt{\left(d+L_{1}\right)^{2}+L_{2}^{2}}} \varphi_{3}(\rho) g(\rho) \rho \mathrm{d} \rho \\
& \varphi_{1}(\rho)=\frac{1}{2}\left(d^{2}-\rho^{2}\right)+L_{2} \sqrt{\rho^{2}-d^{2}} \\
& \varphi_{2}(\rho)=\frac{1}{2}\left(d^{2}-\left(d+L_{1}\right)^{2}\right)+L_{2}\left(\sqrt{\rho^{2}-d^{2}}-\sqrt{\rho^{2}-\left(d+L_{1}\right)^{2}}\right) \\
& \varphi_{3}(\rho)=\frac{1}{2}\left(\rho^{2}+L_{2}^{2}-\left(d+L_{1}\right)^{2}\right)-L_{2} \sqrt{\rho^{2}-\left(d+L_{1}\right)^{2}}
\end{aligned}
$$

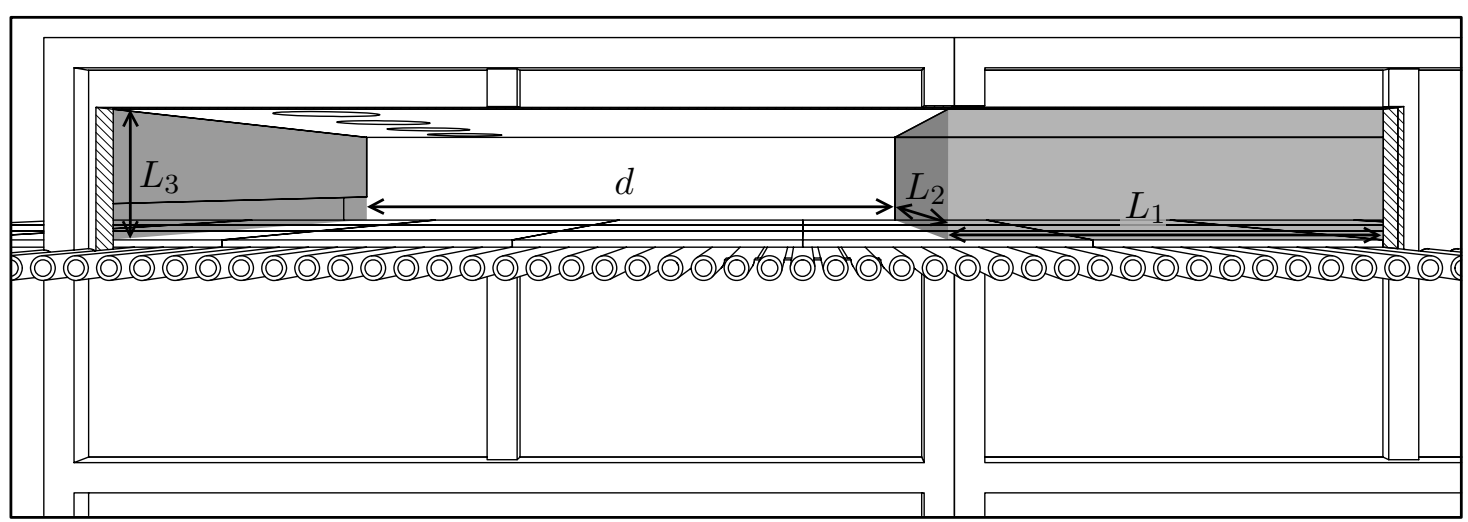

Figura 3.16. Intercambio entre un volumen de gas y la superficie de una pared final. 


$$
\begin{aligned}
g(\rho) & =\frac{4 K}{\pi}\left(\frac{L_{3}^{2}}{\rho^{2}} \frac{\mathrm{e}^{-K \sqrt{\rho^{2}+L_{3}^{2}}}}{\sqrt{\rho^{2}+L_{3}^{2}}}-\frac{K L_{3}}{\rho} \mathrm{S}_{0}\left(K \rho, \operatorname{arctg} \frac{L_{3}}{\rho}\right)+\frac{L_{3}}{K \rho^{3}} \mathrm{~S}_{2}\left(K \rho, \operatorname{arctg} \frac{L_{3}}{\rho}\right)-\right. \\
& \left.-K \Gamma_{-1}\left(K \rho, K \sqrt{\rho^{2}+L_{3}^{2}}\right)\right)
\end{aligned}
$$

3.2.4.3. Intercambio entre un volumen de gas y la superficie de las baldosas en contacto con los rodillos

$$
\begin{aligned}
& \overline{g_{i} s_{j}}= \begin{cases}2\left(\frac{L_{1}}{\delta}-\left\lfloor\frac{x_{i}^{+}}{\delta}+\frac{1}{2}\right\rfloor\right) I_{\infty}+2 \sum_{k=1}^{\frac{x_{i}^{+}}{\delta}+\frac{1}{2}} I_{k} & \text { si están en el mismo sector } \\
\left\lfloor\frac{x_{i}^{+}}{\delta}+\frac{1}{2}\right\rfloor I_{\infty}-\sum_{k=1}^{\frac{x_{i}^{+}}{\delta}+\frac{1}{2}} I_{k} & \text { si están en sectores contiguos } \\
0 & \text { en caso contrario }\end{cases} \\
& x_{i}^{+}=\left(L_{3}-R\right) \sqrt{\left(\frac{\delta}{2 R}\right)^{2}-1} \\
& I_{\infty}=\int_{0}^{L_{3}-R} \int_{t_{1}^{-}}^{t_{1}^{+}\left(x_{i}^{+}, z_{i}\right)}\left(\left(L_{3}-z_{i}\right)\left(x_{i}^{+}+\frac{\delta}{2}\right)+\left(L_{3}-R-z_{i}\right) t_{1}-R \rho\right) g(\rho) \mathrm{d} t_{1} \mathrm{~d} z_{i}+ \\
& +\int_{0}^{L_{3}-R} \int_{t_{1}^{+}\left(x_{i}^{+}, z_{i}\right)}^{t_{1}^{-}}\left(\left(L_{3}-z_{i}\right) \delta-2 R \rho\right) g(\rho) \mathrm{d} t_{1} \mathrm{~d} z_{i}+ \\
& +\int_{0}^{L_{3}-R} \int_{t_{1}^{-}\left(0, z_{i}\right)}^{t_{1}^{+}}\left(\left(L_{3}-z_{i}\right) \frac{\delta}{2}-\left(L_{3}-R-z_{i}\right) t_{1}-R \rho\right) g(\rho) \mathrm{d} t_{1} \mathrm{~d} z_{i}
\end{aligned}
$$

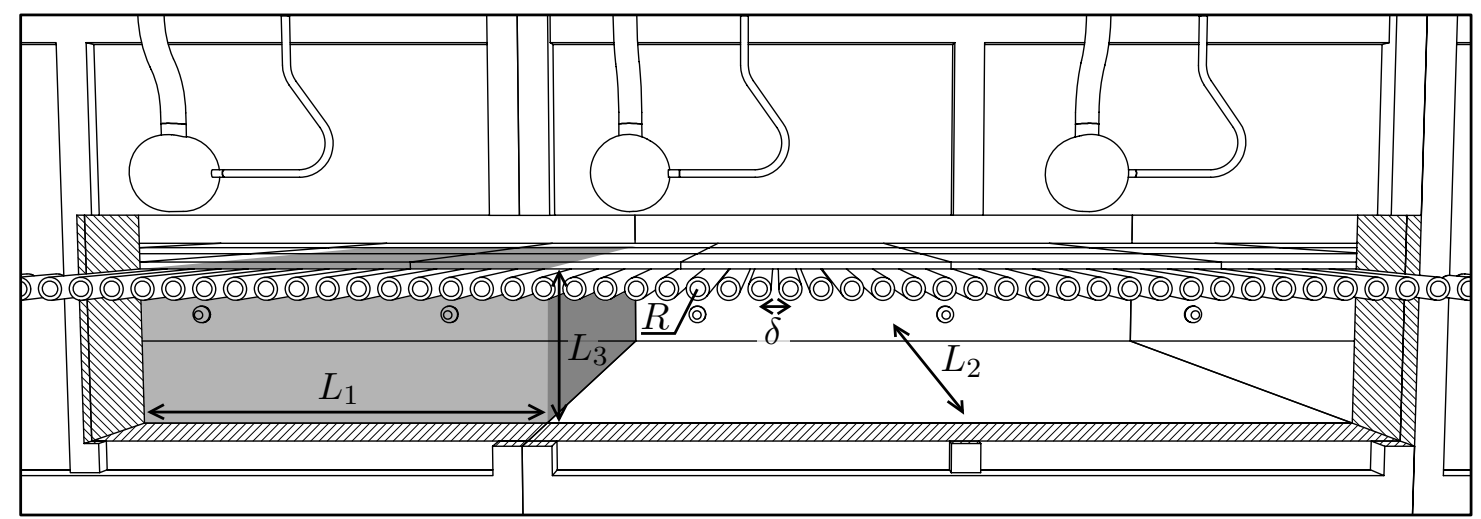

Figura 3.17. Intercambio entre un volumen de gas y la superficie de las baldosas en contacto con los rodillos. 


$$
\begin{aligned}
& I_{k}=\int_{0}^{L_{3}-R} \int_{t_{1}^{-}\left(\left(k-\frac{1}{2}\right) \delta, z_{i}\right)}^{t_{1}^{+}\left(\left(k-\frac{1}{2}\right) \delta, z_{i}\right)}\left(k\left(L_{3}-z_{i}\right) \delta+\left(L_{3}-R-z_{i}\right) t_{1}-R \rho\right) g(\rho) \mathrm{d} t_{1} \mathrm{~d} z_{i}+ \\
& +\int_{0}^{L_{3}-R} \int_{t_{1}^{+}\left(\left(k-\frac{1}{2}\right) \delta, z_{i}\right)}^{t_{1}^{-}\left(0, z_{i}\right)}\left(\left(L_{3}-z_{i}\right) \delta-2 R \rho\right) g(\rho) \mathrm{d} t_{1} \mathrm{~d} z_{i}+ \\
& +\int_{0}^{L_{3}-R} \int_{t_{1}^{-}\left(0, z_{i}\right)}^{t_{1}^{+}}\left(\left(L_{3}-z_{i}\right) \frac{\delta}{2}-\left(L_{3}-R-z_{i}\right) t_{1}-R \rho\right) g(\rho) \mathrm{d} t_{1} \mathrm{~d} z_{i} \\
& \rho=\rho\left(t_{1}, z_{i}\right)=\sqrt{t_{1}^{2}+\left(L_{3}-z_{i}\right)^{2}} \\
& g(\rho)=\frac{2 L_{2}}{\pi \rho}\left(K \frac{\mathrm{e}^{-K \sqrt{t_{2}^{2}+\rho^{2}}}}{\sqrt{t_{2}^{2}+\rho^{2}}}-K^{2} \mathrm{~S}_{0}\left(K \rho, \operatorname{arctg} \frac{L_{2}}{\rho}\right)+\frac{1}{\rho} \mathrm{S}_{2}\left(K \rho, \operatorname{arctg} \frac{L_{2}}{\rho}\right)\right)- \\
& -\frac{2 K^{2}}{\pi} \Gamma_{-1}\left(K \rho, K \sqrt{L_{2}^{2}+\rho^{2}}\right) \\
& t_{1}^{-}\left(x_{i}, z_{i}\right)=-\left(L_{3}-R-z_{i}\right) \frac{x_{i}+\frac{\delta}{2}-R \sqrt{1-\frac{R^{2}-\left(x_{i}+\frac{\delta}{2}\right)^{2}}{\left(L_{3}-R-z_{i}\right)^{2}}}}{L_{3}-2 R-z_{i}} \\
& t_{1}^{+}\left(x_{i}, z_{i}\right)=-\left(L_{3}-R-z_{i}\right) \frac{x_{i}-\frac{\delta}{2}+R \sqrt{1-\frac{R^{2}-\left(x_{i}-\frac{\delta}{2}\right)^{2}}{\left(L_{3}-R-z_{i}\right)^{2}}}}{L_{3}-2 R-z_{i}}
\end{aligned}
$$

\subsubsection{Intercambio entre un volumen de gas y la superficie de los rodillos}

Para el intercambio directo entre un volumen de gas y la superficie de los rodillos se utiliza el cálculo simplificado descrito en §3.2.3.10.

\subsubsection{Algoritmo de integración}

El mayor esfuerzo de cálculo en la aplicación del método zonal se concentra en la evaluación de las DEA. En los orígenes del método, cuando tanto la disponibilidad como la potencia de cálculo de los computadores eran muy limitadas, se reducía el orden de integración de las DEA por medios analíticos hasta conseguir que fuera abordable su integración gráfica [42] o la utilización de fórmulas de cuadratura [85]. Con el desarrollo de los ordenadores personales, este procedimiento tan laborioso ha venido siendo desplazado por el método de Monte Carlo. 
El método de Monte Carlo, aplicado al cálculo de las DEA, consiste en evaluar el integrando de (1.23), (1.26) o (1.29), para dos puntos, seleccionados al azar, situados en sendas zonas entre las que se produce el intercambio. El promedio de los valores obtenidos, multiplicado por las áreas y/o volúmenes de las zonas involucradas, es una estimación del DEA [40, 93, 94]. Algunos autores [95, 96] proponen establecer las posiciones de los puntos emisor y receptor de manera sistemática, en lugar de aleatoria, dividiendo las zonas en pequeñas porciones para las que sea válida una versión discretizada de las DEA. Ambos procedimientos son muy fáciles de programar y permiten calcular las DEA en geometrías complejas.

Desafortunadamente, los métodos indicados son extremadamente ineficientes desde el punto de vista computacional. El método de Monte Carlo parece requerir el estudio de al menos $10^{5}$ «rayos» para conseguir precisiones mínimamente aceptables [94]. El método de subdivisión en pequeñas zonas, considerando que se realizan $N$ divisiones en cada dimensión espacial, tiene asociada una complejidad $\mathcal{O}\left(N^{4}\right)$ para el intercambio entre superficies, que asciende a $\mathcal{O}\left(N^{6}\right)$ para el intercambio entre gases. Además, los dos métodos presentan problemas para los casos de zonas adyacentes o autoirradiadas, en los que el valor del integrando es singular en ciertos puntos.

Los cálculos descritos en esta memoria requieren la evaluación de un gran número de DEA. Una división típica del horno en 60 sectores implica tener que calcular 28348 DEA distintas para cada gas gris involucrado en la WSGG. Además, como se verá en $§ 3.4$ y §3.5, éstas deben calcularse múltiples veces para obtener los resultados. Fue inmediato comprobar la enorme cantidad de tiempo que hubiera sido necesaria para completar los cálculos recogidos en el capítulo 5 si se hubiera utilizado alguno de los métodos de integración indicados.

Por este motivo, al igual que en los trabajos clásicos, se optó por desarrollar las DEA analíticamente para reducir su orden de integración (§A.4). De este modo, se consiguió expresar todas las DEA en términos de integrales simples o dobles. Las integrales resultantes se evaluaron mediante un método de integración específicamente desarrollado que se describe seguidamente. Como resultado, pudieron calcularse las DEA muchos órdenes de magnitud más rápido que con el método de Monte Carlo.

El método de integración utilizado es un algoritmo adaptativo basado en la fórmula abierta de Newton-Côtes de dos puntos:

$$
\int_{a}^{b} f(x) \mathrm{d} x \approx \frac{3}{2} h(f(a+h)+f(b-h))
$$

en la que $h=\frac{b-a}{3}$. En esta fórmula, la función a integrar se aproxima por un polinomio de primer grado que pasa por los puntos interiores contemplados. La ventaja de utilizar fórmulas abiertas es que evitan evaluar los integrandos en los límites de integración. Esto es conveniente para el presente trabajo debido a que las DEA para algunos intercambios son integrales impropias de segunda especie. De esta manera, se consigue solventar el problema del intercambio en la propia zona o entre zonas contiguas.

El algoritmo utilizado se basa en una regla compuesta, lo que significa que el intervalo de integración se divide en subintervalos $[a, b]$ que son en los que se aproxima el valor de la integral por medio de la fórmula (3.80). La integral en el intervalo completo de integración se calcula pues por adición de los valores obtenidos en los subintervalos. 

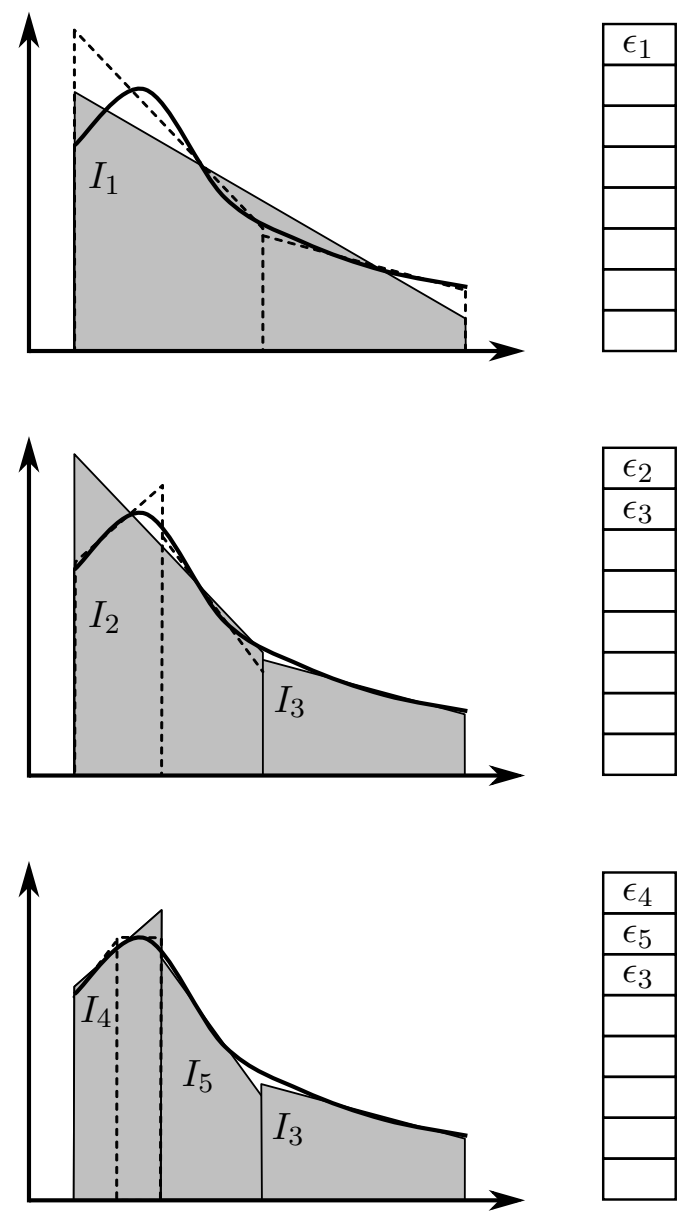

Figura 3.18. Ilustración del algoritmo de integración.

La naturaleza adaptativa del algoritmo consiste en que éste es capaz de encontrar automáticamente una división tal que el error cometido en cada subintervalo sea menor que una cota de error especificada. A tal efecto, se parte de una división inicial grosera, la cual se va refinando sucesivamente de manera que los subintervalos sean más estrechos en aquellas zonas en las que el integrando varía más acusadamente, para lo que se hace uso de una cola de prioridades. Una cola de prioridades es una estructura de datos en la que los elementos se atienden en el orden indicado por una prioridad asociada.

Supóngase que se tiene un subintervalo para el cual una estimación grosera del valor de la integral vendrá dada por:

$$
I_{\mathrm{gros}}=\frac{3}{2} h(f(a+h)+f(b-h))
$$

Este valor podría refinarse dividiendo el subintervalo en dos mitades:

$$
I_{\text {fina }}=\frac{3}{4} h(f(a+h / 2)+f(b-h))+\frac{3}{4} h(f(a+h)+f(b-h / 2))
$$

Los subintervalos en los que existan mayores diferencias entre $I_{\text {gros }}$ y $I_{\text {fina }}$ serán aquellos en los que resulte más prioritario realizar la división, lo que puede gestionarse con una cola 
de prioridades en la que:

$$
\epsilon=\left|I_{\text {gros }}-I_{\text {fina }}\right|
$$

sea el criterio de ordenamiento en la cola.

La aplicación reiterada de este procedimiento es básicamente la esencia del algoritmo. Su progresión se muestra gráficamente en la figura 3.18. Se aprecia cómo se realizan divisiones sucesivamente en aquellas regiones en las que la variación del integrando es más interesante.

\subsubsection{Suavizado de las matrices de DEA}

Las simplificaciones adoptadas para el cálculo de ciertas DEA (§3.2.3.10), junto con la utilización de un coeficiente de absorción medio (§A.7), pueden causar que las relaciones de conservación (1.31) y (1.32) no se verifiquen exactamente (la experiencia ha permitido detectar desviaciones de hasta un $2 \%$ ). Esto es un posible problema, ya que puede originar pérdidas o generaciones incontroladas de energía [97-101]. Existen técnicas para el «suavizado» de las DEA de manera que se cumplan (1.31) y (1.32), simultáneamente con las relaciones de reciprocidad (1.24), (1.27) y (1.30). Aunque ciertos autores [99] sugieren que éste es un procedimiento básicamente arbitrario, la alternativa de que el sistema pueda ser no conservativo es ciertamente peor.

Una de las opciones disponibles para el suavizado de las DEA consiste en modificar mínimamente, en el sentido de los mínimos cuadrados, la estimación inicial de las DEA de manera que se cumplan las relaciones de conservación y de reciprocidad [97]. Como contrapartida de este procedimiento, se tiene el riesgo de que surjan DEA negativas, carentes completamente de sentido físico. A continuación, se describe un método más simple que evita el problema anterior. Este método lo presentó originalmente Leersum [98] y fue modificado posteriormente por Lawson [100] para que las correcciones de las DEA sean proporcionales a su estimación inicial.

1. Se corrigen las filas de $[\overline{\mathbf{s s}}]$ de la manera siguiente, restableciendo la simetría de la matriz:

$$
{\overline{s_{i} s_{j}}}^{\prime}={\overline{s_{j} s_{i}}}^{\prime}=\overline{s_{i} s_{j}} \frac{A_{i}}{\sum_{j=1}^{m} \overline{s_{i} s_{j}}+\sum_{j=1}^{n} \overline{s_{i} g_{j}}}
$$

2. Seguidamente, se corrigen primero las filas de $[\overline{\mathbf{s g}}]$ :

$$
{\overline{s_{i} g_{j}}}^{\prime}=\overline{s_{i} g_{j}} \frac{A_{i}}{\sum_{j=1}^{m} \overline{s_{i} s_{j}}+\sum_{j=1}^{n} \overline{s_{i} g_{j}}}
$$

y después sus columnas:

$$
{\overline{s_{i} g_{j}}}^{\prime}=\overline{s_{i} g_{j}} \frac{4 K V_{j}}{\sum_{i=1}^{n} \overline{g_{i} g_{j}}+\sum_{i=1}^{m} \overline{s_{i} g_{j}}}
$$


3. Finalmente, se corrigen las columnas de [gg], también restableciendo su simetría:

$$
{\overline{g_{i} g_{j}}}^{\prime}={\overline{g_{j} g_{i}}}^{\prime}=\overline{g_{i} g_{j}} \frac{4 K V_{j}}{\sum_{i=1}^{n} \overline{g_{i} g_{j}}+\sum_{i=1}^{m} \overline{s_{i} g_{j}}}
$$

Este proceso se repite las veces necesarias hasta que las relaciones de conservación se satisfagan con la precisión requerida.

\subsection{Balances de energía}

\subsubsection{Numeración de los sectores}

De acuerdo con lo expuesto en §3.1.2, el horno de rodillos se divide longitudinalmente en una serie de sectores. Cada sector está compuesto por diversos elementos - las zonas-. Debe escogerse una nomenclatura que permita distinguir la numeración de los sectores de la propia de las zonas. La nomenclatura seleccionada consiste en identificar a los sectores mediante el índice $(i)$, escrito entre paréntesis. Las magnitudes que correspondan a dicho sector se identifican con un superíndice; por ejemplo, $\dot{V}_{c}^{(i)}$ hará referencia al caudal volumétrico de combustible introducido en el sector $(i)$. Los sectores se numeran en sentido creciente desde la entrada del horno (sentido de avance de las baldosas), habiendo en total $n$ sectores.

\subsubsection{Formulación de los balances}

\subsubsection{Gases}

Cada sector incluye dos volúmenes de gases: uno por encima del plano de baldosas y otro por debajo, entre los cuales no se produce ningún tipo de mezcla (corolario 7.2). En este apartado se formula el balance de energía para uno de estos volúmenes de gases. La formulación sirve tanto para los gases superiores como para los inferiores.

Despreciando la energía en forma de trabajo realizado por o contra el sistema, así como las variaciones de energía cinética y potencial, el balance de energía para un volumen de gas toma la forma general:

$$
\left\{\begin{array}{c}
\text { Flujo de } \\
\text { entalpía } \\
\text { entrante }
\end{array}\right\}+\left\{\begin{array}{c}
\text { Flujo } \\
\text { neto } \\
\text { radiativo }
\end{array}\right\}+\left\{\begin{array}{c}
\text { Flujo } \\
\text { neto } \\
\text { convectivo }
\end{array}\right\}+\left\{\begin{array}{c}
\text { Calor } \\
\text { de } \\
\text { combustión }
\end{array}\right\}=\left\{\begin{array}{c}
\text { Flujo de } \\
\text { entalpía } \\
\text { saliente }
\end{array}\right\}
$$

Para expresar los flujos de entalpía debe conocerse el modo en el que se da la circulación de gases por el sistema (figura 3.19). Al considerar solamente las zonas de calentamiento y cocción, los gases circulan esencialmente en contracorriente con respecto al avance de las piezas. En los sectores en los que existen quemadores, el aire en exceso y los productos de combustión se incorporan a la corriente gaseosa. Asimismo, las baldosas liberan ciertos 


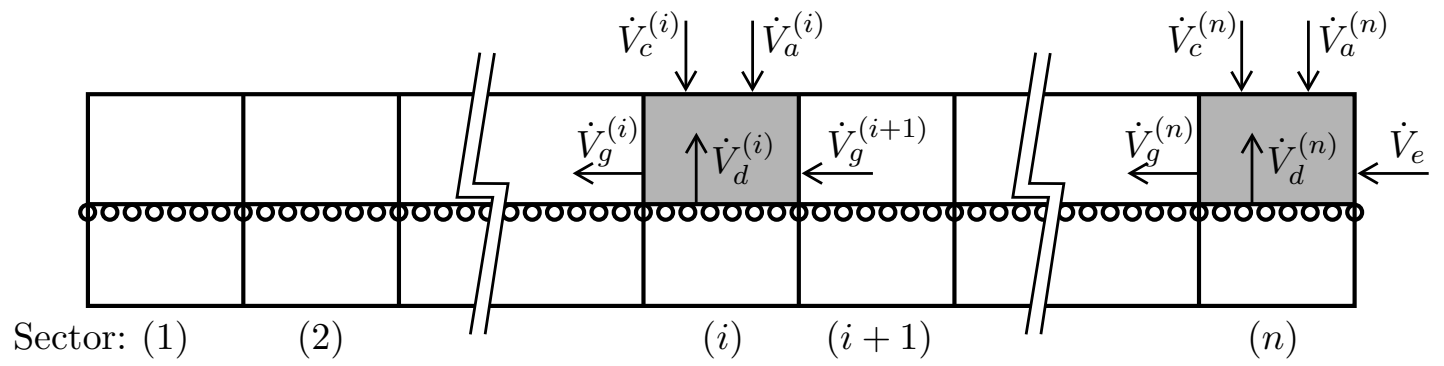

Figura 3.19. Caudales de gases en el horno de rodillos.

productos de descomposición (principalmente $\mathrm{CO}_{2}$ y $\mathrm{H}_{2} \mathrm{O}$ ) en cantidades importantes a medida que avanzan a lo largo del horno.

De acuerdo con las consideraciones anteriores, el flujo de entalpía que entra al volumen de gas es:

$$
\left\{\begin{array}{l}
\text { Flujo de } \\
\text { entalpía } \\
\text { entrante }
\end{array}\right\}=\dot{V}_{g}^{(i+1)} \Delta H_{g}^{\ominus}\left(T_{g, i+1}\right)+\dot{V}_{a}^{(i)} \Delta H_{a}^{\ominus}\left(T_{a}^{(i)}\right)+\dot{V}_{d}^{(i)} \Delta H_{d}^{\ominus}\left(T_{b}^{(i)}\right)
$$

donde $\dot{V}$ son caudales volumétricos y $\Delta H^{\ominus}$ son entalpías específicas expresadas en unidades de energía por unidad de volumen. El flujo de entalpía entrante consta de los términos: $\dot{V}_{g}^{(i+1)} \Delta H_{g}^{\ominus}$ es el flujo de entalpía asociado a los humos provenientes del sector $(i+1)$, $\dot{V}_{a}^{(i)} \Delta H_{a}^{\ominus} \quad$ es el flujo de entalpía de aire comburente introducido en el sector $(i), \mathrm{y}$ $\dot{V}_{d}^{(i)} \Delta H_{d}^{\ominus} \quad$ es el flujo de entalpía de los productos de descomposición de las piezas que se incorporan a la corriente gaseosa a la temperatura de las baldosas $T_{b}^{(i)}$.

La entalpía aportada por el combustible es despreciable.

Teniendo en cuenta la notación propuesta en §3.3.1, la temperatura de los gases podría designarse tanto por $T_{g}^{(i)}$ como por $T_{g, i}$. En esta sección, se preferirá la segunda opción para enfatizar que se trata de la temperatura de una de las zonas. Esta selección requiere que las zonas de gas estén ordenadas como lo están los sectores: en sentido creciente según el avance de las baldosas.

Por la hipótesis 9, en el volumen de gas que se encuentra en el último sector de la zona de calentamiento (sector $n$-ésimo) se incorporará aire de enfriamiento, en lugar de los humos de una combustión anterior (figura 3.19). Así, el término $\dot{V}_{g}^{(i+1)} \Delta H_{g}^{\ominus}\left(T_{g, i+1}\right)$ de la ecuación (3.89) se ve sustituido por $\dot{V}_{e} \Delta H_{e}^{\ominus}\left(T_{e}\right)$, lo que implica conocer el caudal $\dot{V}_{e}$ y la temperatura $T_{e}$ del aire de enfriamiento incorporado a la zona de cocción. Ambas magnitudes pueden determinarse experimentalmente [16].

A diferencia del combustible, el aire comburente puede aportar un cantidad significativa de calor sensible, tanto por los mayores caudales involucrados (en el caso estequiométrico, son 10 veces mayores) como por el hecho de encontrarse a menudo precalentado (a la temperatura $T_{a}^{(i)}$ ) mediante la recuperación de calor del enfriamiento. Los flujos de entalpía del comburente se calculan mediante:

$$
\dot{V}_{a}^{(i)} \Delta H_{a, i}^{\ominus}=\dot{V}_{a}^{(i)}\left(\left(1-x_{\mathrm{O}_{2} a}\right) \Delta H_{\mathrm{N}_{2}}^{\ominus}\left(T_{a}^{(i)}\right)+x_{\mathrm{O}_{2} a} \Delta H_{\mathrm{O}_{2}}^{\ominus}\left(T_{a}^{(i)}\right)\right)
$$


donde $x_{\mathrm{O}_{2} a}$ es la fracción volumétrica de $\mathrm{O}_{2}$ en el aire comburente.

Los gases resultantes de la combustión en el sector $(i)$ lo abandonan a la temperatura de dicho sector, luego:

$$
\left\{\begin{array}{c}
\text { Flujo de } \\
\text { entalpía } \\
\text { saliente }
\end{array}\right\}=\dot{V}_{g}^{(i)} \Delta H_{g}^{\ominus}\left(T_{g, i}\right)
$$

Para calcular los caudales de gases y las entalpías específicas de las corrientes de humos es necesario considerar la combustión. La reacción de combustión de hidrocarburos alifáticos puede escribirse de manera general:

$$
\mathrm{C}_{a} \mathrm{H}_{b}+\left(a+\frac{b}{4}\right) \mathrm{O}_{2} \rightarrow a \mathrm{CO}_{2}+\frac{b}{2} \mathrm{H}_{2} \mathrm{O}
$$

Si se considera que el gas natural está básicamente constituido por metano $\left(\mathrm{CH}_{4}\right)$, etano $\left(\mathrm{C}_{2} \mathrm{H}_{6}\right)$ y propano $\left(\mathrm{C}_{3} \mathrm{H}_{8}\right)$, se tienen los siguientes factores estequiométricos $[13,102]\left(x_{k}\right.$ son fracciones volumétricas/molares de la especie $k$ en el combustible):

$$
V_{\mathrm{O}_{2} \text { mín }}=2 x_{\mathrm{CH}_{4}}+3,5 x_{\mathrm{C}_{2} \mathrm{H}_{6}}+5 x_{\mathrm{C}_{3} \mathrm{H}_{8}}
$$

que representa el volumen oxígeno requerido para completar la combustión de una unidad de volumen de combustible,

$$
V_{\mathrm{CO}_{2}}=x_{\mathrm{CH}_{4}}+2 x_{\mathrm{C}_{2} \mathrm{H}_{6}}+3 x_{\mathrm{C}_{3} \mathrm{H}_{8}}
$$

el volumen de $\mathrm{CO}_{2}$ generado por unidad de volumen de combustible, y:

$$
V_{\mathrm{H}_{2} \mathrm{O}}=2 x_{\mathrm{CH}_{4}}+3 x_{\mathrm{C}_{2} \mathrm{H}_{6}}+4 x_{\mathrm{C}_{3} \mathrm{H}_{8}}
$$

el volumen de $\mathrm{H}_{2} \mathrm{O}$ generado por unidad de volumen de combustible.

A partir de estos factores estequiométricos, pueden calcularse los caudales volumétricos de nitrógeno:

$$
\dot{V}_{\mathrm{N}_{2}}^{(i)}=\left(1-x_{\mathrm{O}_{2} a}\right) \dot{V}_{a}^{(i)}+\dot{V}_{\mathrm{N}_{2}}^{(i+1)}
$$

oxígeno:

$$
\dot{V}_{\mathrm{O}_{2}}^{(i)}=x_{\mathrm{O}_{2} a} \dot{V}_{a}^{(i)}-V_{\mathrm{O}_{2} \min } \dot{V}_{c}^{(i)}+\dot{V}_{\mathrm{O}_{2}}^{(i+1)}
$$

dióxido de carbono:

$$
\dot{V}_{\mathrm{CO}_{2}}^{(i)}=V_{\mathrm{CO}_{2}} \dot{V}_{c}^{(i)}+\dot{V}_{\mathrm{CO}_{2}, d}^{(i)}+\dot{V}_{\mathrm{CO}_{2}}^{(i+1)}
$$

y vapor de agua:

$$
\dot{V}_{\mathrm{H}_{2} \mathrm{O}}^{(i)}=V_{\mathrm{H}_{2} \mathrm{O}} \dot{V}_{c}^{(i)}+\dot{V}_{\mathrm{H}_{2} \mathrm{O}, d}^{(i)}+\dot{V}_{\mathrm{H}_{2} \mathrm{O}}^{(i+1)}
$$

en las que $\dot{V}_{c}^{(i)}$ es el caudal volumétrico de combustible aportado al sector $(i), \dot{V}_{\mathrm{CO}_{2}, d}^{(i)}$ es el $\mathrm{CO}_{2}$ liberado por las baldosas en el sector $(i)$ y $\dot{V}_{\mathrm{H}_{2} \mathrm{O}, d}^{(i)}$ es el $\mathrm{H}_{2} \mathrm{O}$ liberado por las baldosas 


\begin{tabular}{cccc}
$k$ & $b_{0 k} \cdot 10^{-5}\left(\mathrm{~J} \mathrm{~m}^{-3}\right)$ & $b_{1 k} \cdot 10^{-3}\left(\mathrm{~J} \mathrm{~m}^{-3} \mathrm{~K}^{-1}\right)$ & $b_{2 k}\left(\mathrm{~J} \mathrm{~m}^{-3} \mathrm{~K}^{-2}\right)$ \\
\hline $\mathrm{N}_{2}$ & $-3,7105$ & 1,2081 & 0,12147 \\
\hline $\mathrm{O}_{2}$ & $-4,0370$ & 1,2929 & 0,12324 \\
\hline $\mathrm{CO}_{2}$ & $-5,7388$ & 1,7450 & 0,31673 \\
\hline $\mathrm{H}_{2} \mathrm{O}$ & $-4,1047$ & 1,3031 & 0,26889 \\
\hline
\end{tabular}

Tabla 3.1. Coeficientes para el cálculo de las entalpías específicas de los gases de combustión $\left(T_{\text {ref }}=25^{\circ} \mathrm{C}\right)$.

en el sector $(i)$. Al estar expresados todos los caudales en condiciones normales (§3.1.3), el caudal total de humos es la suma de los caudales anteriores:

$$
\dot{V}_{g}^{(i)}=\dot{V}_{\mathrm{N}_{2}}^{(i)}+\dot{V}_{\mathrm{O}_{2}}^{(i)}+\dot{V}_{\mathrm{CO}_{2}}^{(i)}+\dot{V}_{\mathrm{H}_{2} \mathrm{O}}^{(i)}
$$

y también:

$$
\dot{V}_{g}^{(i)} \Delta H_{g}^{\ominus}=\dot{V}_{\mathrm{N}_{2}}^{(i)} \Delta H_{\mathrm{N}_{2}}^{\ominus}+\dot{V}_{\mathrm{O}_{2}}^{(i)} \Delta H_{\mathrm{O}_{2}}^{\ominus}+\dot{V}_{\mathrm{CO}_{2}}^{(i)} \Delta H_{\mathrm{CO}_{2}}^{\ominus}+\dot{V}_{\mathrm{H}_{2} \mathrm{O}}^{(i)} \Delta H_{\mathrm{H}_{2} \mathrm{O}}^{\ominus}
$$

Rhine y Tucker [76] manifiestan reiteradamente que las entalpías específicas de los productos de combustión no varían linealmente con la temperatura. Ahora bien, un polinomio de segundo grado sí que puede representar su dependencia con la temperatura con precisión suficiente:

$$
\Delta H_{k}^{\ominus}\left(T_{g, i}\right) \approx b_{0 k}+b_{1 k} T_{g, i}+b_{2 k} T_{g, i}^{2}
$$

Los coeficientes $b_{0 k}, b_{1 k}$ y $b_{2 k}$ para $\mathrm{N}_{2}, \mathrm{O}_{2}, \mathrm{CO}_{2}$ y $\mathrm{H}_{2} \mathrm{O}$ se obtuvieron a partir de la base de datos termodinámicos del NIST [103] y se detallan en la tabla 3.1.

Teniendo en cuenta la hipótesis 4 , en los sectores en los que se produce la combustión, se liberará en el proceso:

$$
\left\{\begin{array}{c}
\text { Calor } \\
\text { de } \\
\text { combustión }
\end{array}\right\}=-\dot{V}_{c}^{(i)} \Delta_{r} H_{c}^{\ominus}
$$

donde $\Delta_{r} H_{c}^{\ominus}$ es la entalpía de la reacción de combustión del gas natural en el estado base y a la temperatura de referencia. Los suministradores de gas natural suelen expresar el PCI en dichas condiciones, por lo que este dato puede obtenerse por estos medios.

Los flujos de energía radiante se deducen de la exposición presentada en §1.6:

$$
\left\{\begin{array}{c}
\text { Flujo } \\
\text { neto } \\
\text { radiativo }
\end{array}\right\}=\sum_{j=1}^{m} \overrightarrow{S_{j} G_{i}} \sigma T_{j}^{4}+\sum_{j=1}^{n} \overrightarrow{G_{j} G_{i}} \sigma T_{g, j}^{4}-4\left(\sum_{k=0}^{q} a_{k}\left(T_{g, i}\right) \kappa_{k}\right) p_{R}^{(i)} V_{i} \sigma T_{g, i}^{4}
$$

En esta expresión, se ha considerado que el contenido en gases radiantes $\left(\mathrm{CO}_{2}\right.$ y $\left.\mathrm{H}_{2} \mathrm{O}\right)$ en los humos es diferente en cada sector y, por tanto, también lo es la suma de sus presiones parciales $p_{R}^{(i)}$. 
El gas intercambia calor por convección con las superficies con las que está en contacto:

$$
\left\{\begin{array}{c}
\text { Flujo } \\
\text { neto } \\
\text { convectivo }
\end{array}\right\}=\sum_{j \in(i)} A_{j} h_{C}\left(T_{j}-T_{g, i}\right)
$$

donde el sumatorio se extiende a aquellas superficies que se encuentran en el mismo sector (y cerramiento) que el volumen de gas considerado. El coeficiente $h_{C}$ (expresado en $\mathrm{W} \mathrm{m}^{-2} \mathrm{~K}^{-1}$ ) puede estimarse mediante la correlación [104]:

$$
h_{C}=2,3+195 \sqrt{\frac{u_{g}^{(i)}}{T_{g, i}}}
$$

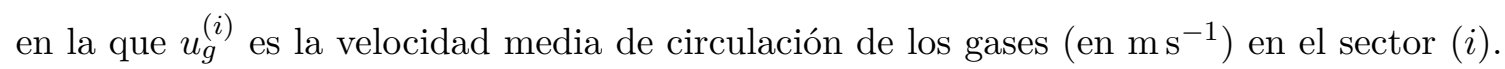
Esta correlación se ha usado satisfactoriamente para obtener el flujo convectivo de calor que llega a las baldosas durante su cocción en hornos de rodillos [27-29]. En este trabajo, este coeficiente se emplea indistintamente para todos los tipos de superficies.

\subsubsection{Baldosas}

A efectos de transferencia radiativa, las baldosas presentes en cada sector se han tratado como una superficie más del sistema. No obstante, las baldosas exhiben características que las diferencian del resto de superficies; a saber:

- Las baldosas avanzan a lo largo del horno, luego existe un flujo de entalpía asociado. En otras palabras, las baldosas son capaces de almacenar calor en su recorrido, lo que es, desde luego, necesario para la operación de cocción.

- Las baldosas experimentan una serie de transformaciones químicas que involucran la generación de especies gaseosas y el consumo (o liberación) del calor de reacción.

- Las baldosas pueden intercambiar energía radiante con las zonas de los cerramientos $\mathcal{S}$ e $\mathcal{I}$. Es decir, las baldosas constan realmente de dos superficies expuestas al horno que corresponden a cada una de sus caras.

- A través del espesor de las baldosas se desarrolla una distribución compleja de temperaturas.

Para poder reproducir este comportamiento, se consideró que las baldosas presentes en cada sector están constituidas por una serie de capas (figura 3.20). La existencia de estas capas añade un grado de abstracción adicional que debe denotarse de una manera identificable. Se ha optado por incluir el índice identificativo de la $j$-ésima capa en el paréntesis utilizado para designar al sector correspondiente. De este modo, la temperatura en el interior de las baldosas se denota con $T_{b}^{(i, j)}$.

El balance de energía para una capa consta de los términos:

$$
\left\{\begin{array}{c}
\text { Flujo de } \\
\text { entalpía } \\
\text { entrante }
\end{array}\right\}+\left\{\begin{array}{c}
\text { Otros } \\
\text { flujos de } \\
\text { energía }
\end{array}\right\}=\left\{\begin{array}{c}
\text { Flujo de } \\
\text { entalpía } \\
\text { saliente }
\end{array}\right\}+\left\{\begin{array}{c}
\text { Calor } \\
\text { de } \\
\text { reacción }
\end{array}\right\}
$$




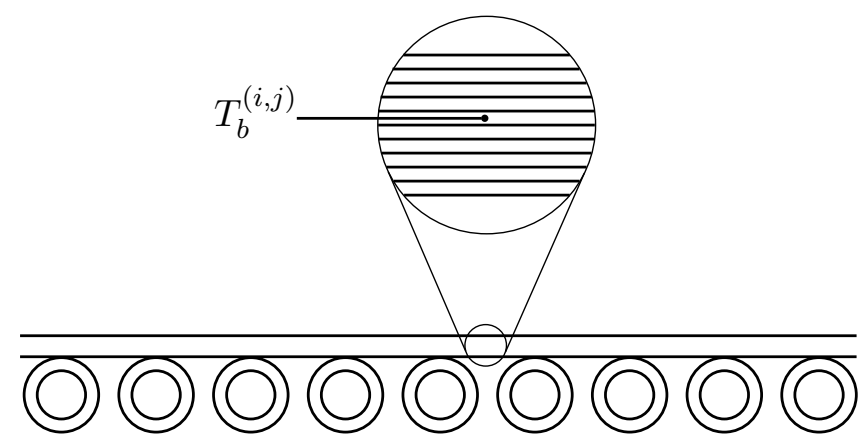

Figura 3.20. Estructura en capas de las baldosas.

El término «otros flujos de energía» depende de si se trata de una capa exterior (expuesta al horno) o interior.

Si se denota con $\dot{m}^{(j)}$ al caudal másico de baldosas crudas dividido por el número de capas, la entrada de entalpía a una capa cualquiera en el sector $(i)$ puede escribirse:

$$
\left\{\begin{array}{l}
\text { Flujo de } \\
\text { entalpía } \\
\text { entrante }
\end{array}\right\}=\dot{m}^{(j)}\left(1-\sum_{k} w_{k} \xi_{k}\left(T_{b}^{(i-1, j)}\right)\right) c_{p b}\left(T_{b}^{(i-1, j)}-T_{\text {ref }}\right)
$$

siendo $c_{p b}$ la capacidad calorífica media de las baldosas y $\xi_{k}$ el grado de avance de la $k$-ésima reacción química referido al componente de referencia, el cual se encuentra en una fracción másica inicial $w_{k}$ en la composición cerámica. $\xi_{k}$ es la fracción másica de componente de referencia que ha reaccionado, de manera que varía desde 0 (cuando la reacción todavía no se ha iniciado) hasta 1 (cuando la reacción se ha completado). Para que la ecuación (3.108) tenga sentido, los productos gaseosos de descomposición de las diferentes reacciones químicas deben escogerse como los respectivos componentes de referencia. De este modo, $\dot{m}^{(j)} w_{k} \xi_{k}$ representa la pérdida de masa asociada a la reacción química.

La entalpía que abandona la capa $(i, j)$ incluye el calor sensible de las baldosas y las especies gaseosas generadas:

$$
\begin{aligned}
\left\{\begin{array}{c}
\text { Flujo de } \\
\text { entalpía } \\
\text { saliente }
\end{array}\right\} & =\dot{m}^{(j)}\left(1-\sum_{k} w_{k} \xi_{k}\left(T_{b}^{(i, j)}\right)\right) c_{p b}\left(T_{b}^{(i, j)}-T_{\text {ref }}\right)+ \\
& +\sum_{k} \dot{V}_{k, d}^{(i, j)} \Delta H_{k}^{\ominus}\left(T_{b}^{(i, j)}\right)
\end{aligned}
$$

El segundo término tiene en cuenta la incorporación de productos a la corriente gaseosa, la cual se supone que tiene lugar inmediatamente. Por simplicidad, se asume que los productos gaseosos generados en las capas por encima del plano medio de las baldosas se incorporan al cerramiento $\mathcal{S}$, mientras que el resto van a parar al cerramiento $\mathcal{I}$. El caudal volumétrico de la especie $k$ puede calcularse mediante:

$$
\dot{V}_{k, d}^{(i, j)}=\frac{\dot{m}^{(j)} w_{k}}{\rho_{k}}\left(\xi_{k}\left(T_{b}^{(i, j)}\right)-\xi_{k}\left(T_{b}^{(i-1, j)}\right)\right)
$$

donde $\rho_{k}$ es la densidad de la especie $k$ en estado gaseoso en condiciones normales. 
Aparte del calor sensible, deben tenerse en cuenta las transformaciones químicas que experimenta la composición durante la cocción, con el consiguiente consumo o liberación del calor de reacción. El calor de reacción puede expresarse en términos de la entalpía de reacción en el estado base y a la temperatura de referencia:

$$
\left\{\begin{array}{c}
\text { Calor } \\
\text { de } \\
\text { reacción }
\end{array}\right\}=\dot{m}^{(j)} \sum_{k} \frac{w_{k}}{\rho_{k}}\left(\xi_{k}\left(T_{b}^{(i, j)}\right)-\xi_{k}\left(T_{b}^{(i-1, j)}\right)\right) \Delta_{r} H_{k}^{\ominus}
$$

Es importante señalar que en esta expresión, a diferencia de (1.16), el calor de reacción viene expresado en unidades de energía por unidad de volumen en condiciones normales, por coherencia con §3.3.2.1.

Para el objeto del presente trabajo, las reacciones más importantes son la deshidroxilación de los minerales arcillosos y la descomposición de carbonatos, ambas endotérmicas. Las otras muchas transformaciones fisico-químicas que tienen lugar en la composición cerámica no son relevantes desde el punto de vista térmico [21,23,27], ni tampoco tienen asociada la liberación de cantidades importantes de gases.

Como se ha indicado anteriormente, los componentes de referencia de las reacciones químicas deben ser los productos gaseosos. La deshidroxilación de los minerales arcillosos libera $\mathrm{H}_{2} \mathrm{O}$, mientras que la descomposición de los carbonatos da como producto $\mathrm{CO}_{2}$. Las cantidades generadas no son en absoluto despreciables. Por ejemplo, para una composición de azulejo ( $13 \%$ de contenido en carbonatos y $10 \%$ de pérdidas por calcinación) que involucre el consumo medio de energía en la cocción indicado en $\S 1.2$, el $\mathrm{CO}_{2}$ y el $\mathrm{H}_{2} \mathrm{O}$ liberados por las reacciones químicas supondrían aproximadamente el $30 \%$ del total de $\mathrm{CO}_{2}$ y $\mathrm{H}_{2} \mathrm{O}$ en los gases de combustión.

La relación entre el grado de avance y la temperatura puede describirse mediante un modelo sencillo [27]:

$$
\xi_{k}=\left(3 \Theta_{k}^{2}-2 \Theta_{k}^{3}\right)\left(\mathrm{H}\left(\Theta_{k}\right)-\mathrm{H}\left(\Theta_{k}-1\right)\right)+\mathrm{H}\left(\Theta_{k}-1\right)
$$

donde $\mathrm{H}(\bullet)$ es la función de Heaviside (escalón unidad) y $\Theta_{k}$ ha sido definida previamente (ecuación (1.17) en la página 20), pero se repite aquí por conveniencia:

$$
\Theta_{k}=\frac{T_{b}^{(i, j)}-T_{k}^{-}}{T_{k}^{+}-T_{k}^{-}}
$$

Las temperaturas $T_{k}^{-}$y $T_{k}^{+}$se interpretan como las temperaturas de inicio y finalización de la reacción química, respectivamente. Los valores de estos parámetros para las reacciones químicas consideradas fueron determinados experimentalmente por Cantavella [27] (tabla 3.2).

Dependiendo de si se trata de una capa interior o exterior, el resto de términos del balance de energía son diferentes. Para las capas interiores, debe contemplarse el intercambio de calor por conducción con las capas adyacentes:

$$
\left\{\begin{array}{c}
\text { Otros } \\
\text { flujos de } \\
\text { energía }
\end{array}\right\}=\left\{\begin{array}{c}
\text { Flujo } \\
\text { neto } \\
\text { conductivo }
\end{array}\right\}
$$




\begin{tabular}{ccccc} 
Reacción & $k$ & $T_{k}^{-}\left({ }^{\circ} \mathrm{C}\right)$ & $T_{k}^{+}\left({ }^{\circ} \mathrm{C}\right)$ & $\Delta_{r} H_{k}^{\ominus}\left(\mathrm{kJ} \mathrm{m}^{-3}\right)$ \\
\hline Deshidroxilación de minerales arcillosos & $\mathrm{H}_{2} \mathrm{O}$ & 510 & 700 & 5000 \\
\hline Descomposición de carbonatos & $\mathrm{CO}_{2}$ & 700 & 900 & 8500 \\
\hline
\end{tabular}

Tabla 3.2. Parámetros de la ecuación (3.112) [20,27].

la cual puede describirse mediante la ley de Fourier:

$$
\left\{\begin{array}{c}
\text { Flujo } \\
\text { neto } \\
\text { conductivo }
\end{array}\right\}=A_{i} \frac{\lambda_{b}}{\ell_{b}^{(j)}}\left(T_{b}^{(i, j-1)}-T_{b}^{(i, j)}\right)+A_{i} \frac{\lambda_{b}}{\ell_{b}^{(j)}}\left(T_{b}^{(i, j+1)}-T_{b}^{(i, j)}\right)
$$

donde $\lambda_{b}$ es la conductividad térmica de las baldosas y $\ell_{b}^{(j)}$ es el espesor de las capas.

Las capas exteriores de las baldosas son zonas de superficie, por lo que debe considerarse la transmisión de calor por convección y radiación:

$$
\left\{\begin{array}{c}
\text { Otros } \\
\text { flujos de } \\
\text { energía }
\end{array}\right\}=\left\{\begin{array}{c}
\text { Flujo } \\
\text { neto } \\
\text { conductivo }
\end{array}\right\}+\left\{\begin{array}{c}
\text { Flujo } \\
\text { neto } \\
\text { radiativo }
\end{array}\right\}+\left\{\begin{array}{c}
\text { Flujo } \\
\text { neto } \\
\text { convectivo }
\end{array}\right\}
$$

Al tratarse de capas exteriores, sólo se produce intercambio conductivo con otra capa; por ejemplo, para la capa $(i, 1)$ :

$$
\left\{\begin{array}{c}
\text { Flujo } \\
\text { neto } \\
\text { conductivo }
\end{array}\right\}=A_{i} \frac{\lambda_{b}}{\ell_{b}^{(j)}}\left(T_{b}^{(i, 2)}-T_{i}\right)
$$

El flujo radiativo puede escribirse de la manera indicada en $§ 1.6$ :

$$
\left\{\begin{array}{c}
\text { Flujo } \\
\text { neto } \\
\text { radiativo }
\end{array}\right\}=\sum_{j=1}^{m} \overrightarrow{S_{j} S_{i}} \sigma T_{j}^{4}+\sum_{j=1}^{n} \overrightarrow{G_{j} S_{i}} \sigma T_{g, j}^{4}-A_{i} \varepsilon_{i} \sigma T_{i}^{4}
$$

El flujo convectivo debe ser consistente con (3.105):

$$
\left\{\begin{array}{c}
\text { Flujo } \\
\text { neto } \\
\text { convectivo }
\end{array}\right\}=A_{i} h_{C}\left(T_{g}^{(i)}-T_{i}\right)
$$

En las tres últimas ecuaciones se ha utilizado la notación $T_{i}$, en lugar de $T_{b}^{(i, 1)}$, ya que en estos casos se trata de las temperaturas de ciertas zonas.

\subsubsection{Paredes}

Esta sección abarca los balances de energía para un conjunto relativamente amplio de superficies cuya característica distintiva es que pueden conducir calor a su través y transmitirlo al ambiente circundante. El balance de energía para una de estas superficies puede 
escribirse:

$$
\left\{\begin{array}{c}
\text { Flujo } \\
\text { neto } \\
\text { radiativo }
\end{array}\right\}+\left\{\begin{array}{c}
\text { Flujo } \\
\text { neto } \\
\text { convectivo }
\end{array}\right\}=\left\{\begin{array}{c}
\text { Pérdidas } \\
\text { por } \\
\text { conducción }
\end{array}\right\}
$$

El término «pérdidas por conducción» puede expresarse mediante la ecuación (1.7):

$$
\left\{\begin{array}{c}
\text { Pérdidas } \\
\text { por } \\
\text { conducción }
\end{array}\right\}=A_{i} U\left(T_{i}-T_{\infty}\right)
$$

siendo $U$ el coeficiente global de transmisión de calor:

$$
\frac{1}{U}=\frac{\ell}{\lambda}+\frac{A_{i} / A_{o}}{h_{C o}+h_{R o}}
$$

expresión que ya se ha descrito anteriormente (ecuación (1.9) en la página 19).

La conductividad térmica de los materiales refractarios y aislantes empleados en las paredes del horno está disponible a través de diversas fuentes [76, 105], incluidos los propios fabricantes de estos materiales. La emisividad de la superficie exterior de las paredes (necesaria para estimar $h_{R o}$ ) puede determinarse experimentalmente o incluso puede considerarse que estas superficies son negras sin cometer mucho error. Para la estimación de $h_{C o}$ pueden utilizarse correlaciones semiempíricas que relacionan el módulo de Nusselt con los módulos de Rayleigh y de Prandtl (estos módulos se definen en §1.5.2):

- Para paredes verticales se ha seleccionado la relación de Churchill y Chu [106]:

$$
\mathrm{Nu}^{\frac{1}{2}}=0,825+\frac{0,387 \mathrm{Ra}^{\frac{1}{6}}}{\left(1+\left(\frac{0,492}{\mathrm{Pr}}\right)^{\frac{9}{16}}\right)^{\frac{8}{27}}}
$$

- Para paredes horizontales que se enfrían por la parte superior se ha escogido la relación de Al-Arabi y El-Riedy [107]:

$$
\mathrm{Nu}= \begin{cases}0,7 \mathrm{Ra}^{\frac{1}{4}} & \text { si } \mathrm{Ra} \leq 2 \cdot 10^{7} \\ 0,155 \mathrm{Ra}^{\frac{1}{3}} & \text { si } \mathrm{Ra}>2 \cdot 10^{7}\end{cases}
$$

- Para paredes horizontales que se enfrían por la parte inferior se ha recurrido a la expresión clásica de Fishenden y Saunders [108]:

$$
\mathrm{Nu}=0,27 \mathrm{Ra}^{\frac{1}{4}}
$$

Al igual que las capas exteriores de las baldosas, las superficies internas de las paredes del horno pueden intercambiar calor con las otras zonas por radiación:

$$
\left\{\begin{array}{c}
\begin{array}{c}
\text { Flujo } \\
\text { neto } \\
\text { radiativo }
\end{array}
\end{array}\right\}=\sum_{j=1}^{m} \overrightarrow{S_{j} S_{i}} \sigma T_{j}^{4}+\sum_{j=1}^{n} \overrightarrow{G_{j} S_{i}} \sigma T_{g, j}^{4}-A_{i} \varepsilon_{i} \sigma T_{i}^{4}
$$

y con los gases con los que están en contacto por convección:

$$
\left\{\begin{array}{c}
\text { Flujo } \\
\text { neto } \\
\text { convectivo }
\end{array}\right\}=A_{i} h_{C}\left(T_{g}^{(i)}-T_{i}\right)
$$




\subsubsection{Rodillos}

Para plantear los balances de energía para los rodillos se ha considerado, por una parte, que no existen pérdidas de energía por conducción a su través y, por otra parte, que la transferencia de energía entre los rodillos y las baldosas, por el hecho de encontrarse en contacto físico, es despreciable. El primer supuesto se discute más adelante, en el capítulo 4, ya que está basado en evidencias experimentales. En cuanto a la hipótesis de que no existe transferencia de energía por contacto entre las baldosas y los rodillos, ya se ha mencionado en $§ 1.5 .4$ que, en caso de existir, no debería ser de una magnitud muy significativa. Efectivamente, se han publicado estudios en los que se comprueba que las teorías disponibles predicen flujos de calor muy superiores a los observados experimentalmente [35,109]. Estas discrepancias se atribuyen a la rugosidad de los materiales, a pesar de haberse estudiado superficies relativamente lisas. En el caso del contacto entre las baldosas y los rodillos, cabría esperar flujos de calor incluso más modestos, debido al relieve de la costilla de las baldosas.

Teniendo en cuenta las consideraciones anteriores, el balance de energía para los rodillos queda simplemente:

$$
\left\{\begin{array}{c}
\text { Flujo } \\
\text { neto } \\
\text { radiativo }
\end{array}\right\}+\left\{\begin{array}{c}
\text { Flujo } \\
\text { neto } \\
\text { convectivo }
\end{array}\right\}=0
$$

en la que surgen los habituales términos:

$$
\left\{\begin{array}{c}
\text { Flujo } \\
\text { neto } \\
\text { radiativo }
\end{array}\right\}=\sum_{j=1}^{m} \overrightarrow{S_{j} S_{i}} \sigma T_{j}^{4}+\sum_{j=1}^{n} \overrightarrow{G_{j} S_{i}} \sigma T_{g, j}^{4}-A_{i} \varepsilon_{i} \sigma T_{i}^{4}
$$

para la radiación, y:

$$
\left\{\begin{array}{c}
\text { Flujo } \\
\text { neto } \\
\text { convectivo }
\end{array}\right\}=A_{i} h_{C}\left(T_{g}^{(i)}-T_{i}\right)
$$

para la convección.

\subsection{Resolución de los balances de energía}

\subsubsection{Planteamiento}

En esta sección se describirá un método numérico para el cálculo de las temperaturas de las zonas, lo que implica conocer:

- la geometría del horno y las propiedades de las paredes y los rodillos,

- la producción, composición y propiedades de las baldosas, y

- los caudales de combustible y de comburente (ver, no obstante, §3.5). 
Si se dispone de toda esta información, pueden realizarse los cálculos de combustión, calcularse las DEA y las TEA, etc. para llegar a un sistema de ecuaciones no lineales - los balances de energía - en el que las incógnitas son las temperaturas de las zonas.

Se supondrá que se parte de una estimación inicial de las temperaturas de las zonas (en el programa informático, se utilizó la temperatura ambiente $T_{\infty}$ ) de manera que los parámetros que dependen de la temperatura (por ejemplo, las DFA) tienen un valor numérico tentativo.

Dada la complejidad del problema, se descartó de inmediato la utilización de un método genérico de resolución de sistemas de ecuaciones no lineales, como puede ser el método de Newton-Raphson. En su lugar, se desarrolló un algoritmo propio, el cual cuenta con múltiples niveles de iteración, por lo que es mejor describirlo incrementalmente. Así, en los apartados siguientes, se describe un algoritmo base de resolución de los balances, al que se van añadiendo gradualmente características adicionales, para dar lugar al algoritmo utilizado en su forma completa.

\subsubsection{Resolución de un cerramiento}

En esta sección se considerará un único cerramiento, entendido como un sistema cerrado para la radiación térmica, que podría tratarse tanto del cerramiento $\mathcal{S}$ como del $\mathcal{I}$. En primer lugar, se abordará la transferencia de energía radiante en el cerramiento. Para ello, es práctico escribir las DFA en forma matricial: $[\overrightarrow{\mathbf{S S}}],[\overrightarrow{\mathbf{S G}}],[\overrightarrow{\mathbf{G S}}]$ y $[\overrightarrow{\mathbf{G G}}]$. A partir de estas matrices, puede componerse la siguiente matriz de DFA generalizadas (al estilo de las referencias [61-64], posiblemente inspiradas por la formulación de Naraghi y Chung [110]):

$$
[\overrightarrow{\mathbf{Z Z}}]=\left[\begin{array}{ll}
{[\overrightarrow{\mathbf{S S}}]} & {[\overrightarrow{\mathbf{G G}}]} \\
{[\overrightarrow{\mathbf{G S}}]} & {[\overrightarrow{\mathbf{G G}}]}
\end{array}\right]
$$

El producto de la matriz $[\overrightarrow{\mathbf{Z Z}}]$ por un vector de poderes emisivos oportunamente ordenados daría como resultado un vector con los flujos de energía radiante entrantes a cada una de las zonas.

Para resolver los balances de energía, Shen y colaboradores [111] propusieron linealizar los poderes emisivos de la manera siguiente:

$$
E_{i}=\sigma \hat{T}_{i}^{3} T_{i}
$$

donde el circunflejo $\left({ }^{\wedge}\right)$ indica que dicha temperatura es estimada. El objeto de aplicar esta linealización es transformar el sistema en uno lineal de la forma:

$$
[\hat{\mathbf{M}}]\{\mathbf{T}\}=\{\hat{\mathbf{Q}}\}
$$

a resolver múltiples veces, hasta que se alcance la convergencia.

La matriz $[\overrightarrow{\mathbf{Z Z}}]$ es un buen punto de partida para construir la matriz $[\hat{\mathbf{M}}]$. Es fácil comprobar que la siguiente matriz, multiplicada por el vector de temperaturas de las zonas $\{\mathbf{T}\}$, da 
como resultado un vector de flujos netos radiativos para cada zona:

$$
[\hat{\mathbf{M}}]=\left[\begin{array}{cccc}
-\sum_{j \neq 1} \overrightarrow{Z_{1} Z_{j}} \sigma \hat{T}_{1}^{3} & \overrightarrow{Z_{2} Z_{1}} \sigma \hat{T}_{2}^{3} & \cdots & \overrightarrow{Z_{N} Z_{1}} \sigma \hat{T}_{N}^{3} \\
\overrightarrow{Z_{1} Z_{2}} \sigma \hat{T}_{1}^{3} & -\sum_{j \neq 2} \overrightarrow{Z_{2} Z_{j}} \sigma \hat{T}_{2}^{3} & \cdots & \overrightarrow{Z_{N} Z_{2}} \sigma \hat{T}_{N}^{3} \\
\vdots & \vdots & \ddots & \vdots \\
\overrightarrow{Z_{1} Z_{N}} \sigma \hat{T}_{1}^{3} & \overrightarrow{Z_{2} Z_{N}} \sigma \hat{T}_{2}^{3} & \cdots & -\sum_{j \neq N} \overrightarrow{Z_{N} Z_{j}} \sigma \hat{T}_{N}^{3}
\end{array}\right]
$$

en la que $N=m+n$.

Un posible planteamiento para obtener un sistema de la forma de (3.133) podría consistir en englobar en el vector $\{\hat{\mathbf{Q}}\}$ el resto de términos (distintos de los flujos radiativos) de los balances de energía. No obstante, para mejorar la convergencia, es apropiado incluir en la matriz $[\hat{\mathbf{M}}]$ al menos los flujos de entalpía de los humos. Para ello, las entalpías específicas de los gases también pueden escribirse en versión linealizada:

$$
\Delta H_{k}^{\ominus}\left(T_{g, i}\right) \approx b_{0 k}+\left(b_{1 k}+b_{2 k} \hat{T}_{g, i}\right) T_{g, i}
$$

de manera que los términos de la forma $\dot{V}_{k}\left(b_{1 k}+b_{2 k} \hat{T}_{g, i}\right)$ se incorporan en las posiciones oportunas de $[\hat{\mathbf{M}}]$, mientras que los términos $\dot{V}_{k} b_{0 k}$ se incluyen en el vector $\{\hat{\mathbf{Q}}\}$. Asimismo, el tratamiento de los balances de energía para las baldosas merece una especial atención, por lo que se describe en el apartado específico §3.4.3.

Una vez construidos $[\hat{\mathbf{M}}]$ y $\{\hat{\mathbf{Q}}\}$, debería resolverse la ecuación (3.133) de manera iterativa. Sin embargo, este esquema es divergente. La convergencia se consigue introduciendo un factor de relajación $\alpha$ en el esquema iterativo:

$$
\{\mathbf{T}\}=\alpha[\hat{\mathbf{M}}]^{-1}\{\hat{\mathbf{Q}}\}+(1-\alpha)\{\hat{\mathbf{T}}\}
$$

en la que $\{\hat{\mathbf{T}}\}$ es el vector de temperaturas estimadas (resultado de la iteración anterior). El uso de factores de relajación es desafortunado. Un valor demasiado elevado puede causar la divergencia, mientras que un valor demasiado pequeño hace que el proceso iterativo progrese muy lentamente. Se ha ideado un método que selecciona un factor de relajación apropiado en cada iteración, basándose en el estudio del residuo de la ecuación (3.133):

$$
e=\|[\hat{\mathbf{M}}]\{\mathbf{T}\}-\{\hat{\mathbf{Q}}\}\|
$$

Se considera que la variación del residuo con el factor de relajación puede aproximarse mediante una parábola:

$$
e \approx b_{0}+b_{1} \alpha+b_{2} \alpha^{2}
$$

cuyos coeficientes se determinan evaluando el residuo para tres valores de $\alpha$ (concretamente, se utiliza $\alpha=0, \alpha=0,5$ y $\alpha=1)$. Haciendo $\frac{\mathrm{d} e}{\mathrm{~d} \alpha}=0$ se obtiene el valor del factor de relajación que minimiza el residuo:

$$
\alpha_{\text {ópt }}=\frac{b_{1}}{2 b_{2}}
$$


Como precaución adicional, se define un valor umbral $\alpha_{\text {mín }}$, tal que:

$$
\alpha= \begin{cases}\alpha_{\text {ópt }} & \text { si } \alpha_{\text {ópt }} \geq \alpha_{\text {mín }} \\ \alpha_{\text {mín }} & \text { si } \alpha_{\text {ópt }}<\alpha_{\text {mín }}\end{cases}
$$

para garantizar la progresión del algoritmo.

\subsubsection{Tratamiento del balance para las baldosas}

El algoritmo presentado hasta el momento permite resolver los balances de energía para un cerramiento. Sin embargo, de acuerdo con el corolario 7.1, en ausencia de cortafuegos intermedios (los cuales se contemplan en §3.4.4), el horno de rodillos está compuesto por dos cerramientos: el $\mathcal{S}$ y el $\mathcal{I}$. Los balances de energía para estos cerramientos no son independientes, ya que pueden intercambiar energía por conducción a través de las baldosas. Queda pendiente describir cómo se consigue el acoplamiento entre cerramientos y el cálculo de la distribución interna de temperaturas en las baldosas. En este apartado se abordará este segundo punto.

Para calcular la distribución interna de temperatura en las baldosas, se escribe el balance de energía para las capas interiores de las mismas en la forma:

$$
-\mathrm{Fo} T_{b}^{(i, j-1)}+\left(1-\hat{\Xi}^{(i, j)}+2 \mathrm{Fo}\right) T_{b}^{(i, j)}-\mathrm{Fo}_{b}^{(i, j+1)}=\hat{T}_{*}^{(i, j)}
$$

siendo:

$$
\begin{aligned}
& \text { Fo }=\frac{\lambda_{b} A_{i}}{\dot{m}^{(j)} c_{p b} \ell_{b}^{(j)}} \\
& \begin{aligned}
\hat{\Xi}^{(i, j)} & =\sum_{k} w_{k} \xi_{k}\left(\hat{T}_{b}^{(i, j)}\right) \\
\hat{T}_{*}^{(i, j)} & =\left(1-\hat{\Xi}^{(i-1, j)}\right)\left(\hat{T}_{b}^{(i-1, j)}-T_{\mathrm{ref}}\right)+\left(1-\hat{\Xi}^{(i, j)}\right) T_{\mathrm{ref}}- \\
& -\frac{1}{c_{p b}} \sum_{k} \frac{w_{k}}{\rho_{k}}\left(\xi_{k}\left(\hat{T}_{b}^{(i, j)}\right)-\xi_{k}\left(\hat{T}_{b}^{(i-1, j)}\right)\right)\left(\Delta H_{k}^{\ominus}\left(\hat{T}_{b}^{(i, j)}\right)+\Delta_{r} H_{k}^{\ominus}\right)
\end{aligned}
\end{aligned}
$$

Planteando estas ecuaciones para todas las capas interiores de las baldosas se llega a un sistema tridiagonal de ecuaciones que puede resolverse mediante el algoritmo 4.3.6 de la referencia [112], ya que la matriz del sistema es simétrica, definida positiva y con diagonal dominante. Las temperaturas de la primera y última capas, expuestas a sendos cerramientos $(\mathcal{S}$ e $\mathcal{I}$, respectivamente), se obtienen de la resolución de los correspondientes balances de energía.

Existe otra peculiaridad de las baldosas que tiene relevancia en la resolución de los balances de energía. Las baldosas modifican de manera significativa la composición de los gases, al liberar especies radiantes $\left(\mathrm{CO}_{2}\right.$ y $\left.\mathrm{H}_{2} \mathrm{O}\right)$ en cantidades importantes en su recorrido a lo largo del horno. Estas especies se liberan a la atmósfera del horno cuando las baldosas alcanzan una determinada temperatura, pero la temperatura de las baldosas es una incógnita. Esto significa que, aún manteniendo fijos los caudales de combustible y comburente, la composición de los humos variará en el proceso de resolución de los balances, en función 


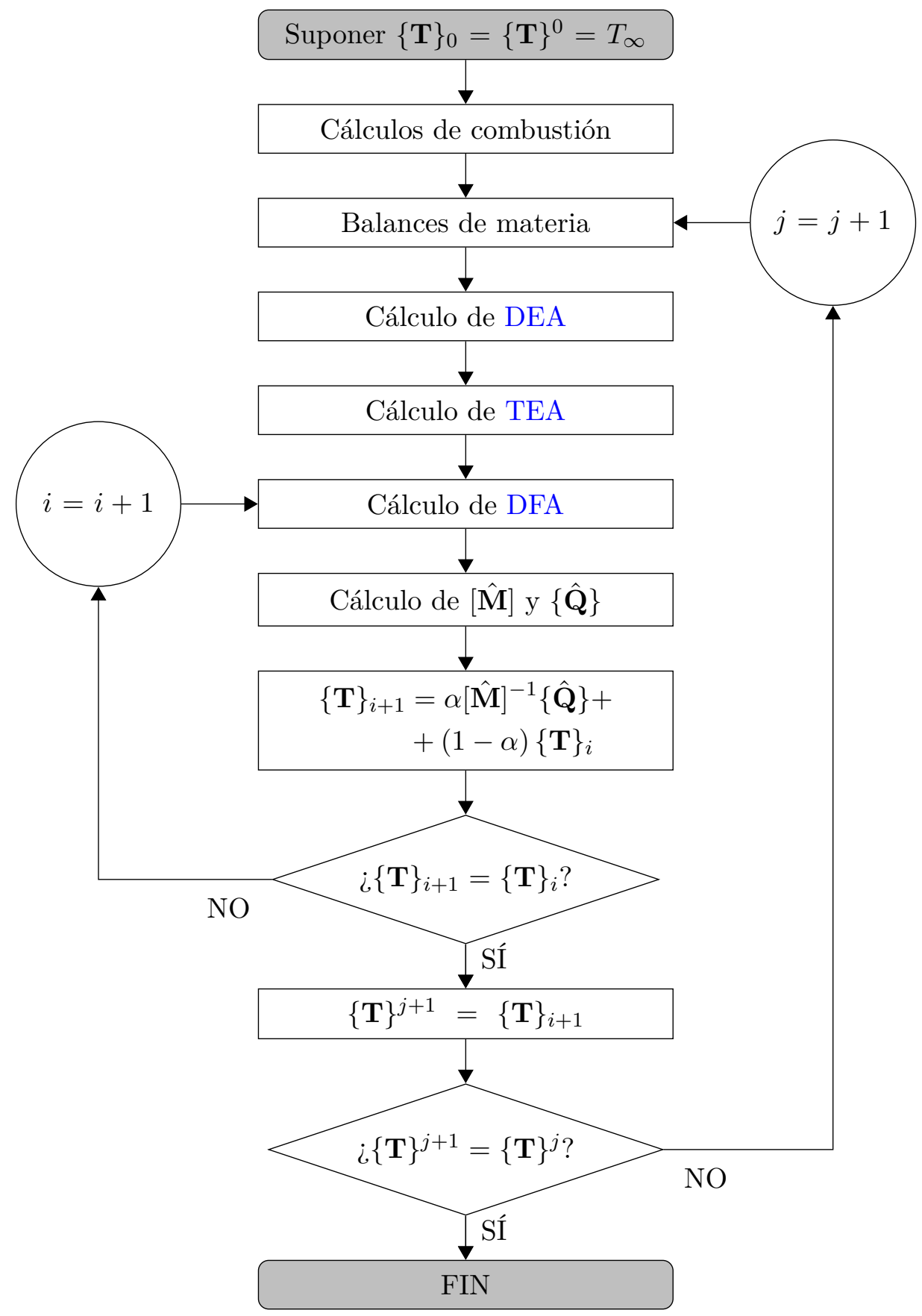

Figura 3.21. Utilización de dos niveles de iteración en la resolución de los balances de energía.

de la temperatura interna de las baldosas. Las DEA y, por extensión, las DFA dependen directamente del contenido en gases radiantes en los humos, pero sería ineficiente actualizar las DEA/TEA en cada iteración. Para minimizar el cálculo de DEA, es apropiado utilizar dos niveles de iteración. Este concepto se ilustra en la figura 3.21. 
En el algoritmo presentado en la figura 3.21 se separa una iteración interna, para la resolución de los balances en un cerramiento, de un bucle exterior en el que se actualizan las DEA y TEA. El razonamiento subyacente consiste en que sería acertado recalcular la atmósfera del horno únicamente cuando se dispone de una estimación de la temperatura de las baldosas razonablemente buena. En efecto, la convergencia del bucle exterior es relativamente rápida: apenas se requieren unas pocas iteraciones, de manera que el número de DEA a calcular se mantiene relativamente bajo.

\subsubsection{Acoplamiento de cerramientos}

En este apartado se detalla cómo se consigue la consistencia de los balances de energía de los distintos cerramientos. Éste es el momento oportuno para presentar el tratamiento de los cortafuegos, los cuales son básicamente barreras físicas que cierran (considerablemente) la sección de paso de los gases. La abundancia y localización de estos dispositivos varía según el horno considerado, aunque existen posiciones en las que siempre hay un cortafuegos (o un elemento análogo como, por ejemplo, la separación del calentamiento y el enfriamiento).

De acuerdo con las hipótesis adoptadas para el desarrollo del modelo, los cortafuegos impiden la transferencia de radiación térmica entre las zonas situadas en las respectivas regiones que éstos delimitan (hipótesis 8). Así pues, el canal superior del horno estará compuesto por una serie de cerramientos $\mathcal{S}$, mientras que el canal inferior contará con varios cerramientos $\mathcal{I}$ (corolario 8.1). La transferencia de energía entre los cerramientos de un canal se producirá solamente por flujo de materiales (baldosas y gases) y por conducción a través de los cortafuegos.

El acoplamiento de los cerramientos se consigue, básicamente, por medio de una secuencia iterativa en la que los balances de energía correspondientes a cada uno de los cerramientos se van resolviendo sucesivamente. Conseguir que este procedimiento converja a la solución no es una tarea sencilla. Además, el algoritmo debe acumular las pautas indicadas hasta el momento. Es complicado especificar el algoritmo completo en términos de un diagrama de flujo. Sin embargo, el lenguaje de programación C++ [113,114], utilizado para implementar el modelo en el ordenador, permite expresar el algoritmo de una manera extraordinariamente concisa. Por este motivo, se ha optado por detallar el listado de instrucciones que componen el código fuente para poder explicar, finalmente, los pasos de cálculo que componen el algoritmo realmente utilizado (figura 3.22).

La función (rutina de cálculo) Resuelve, definida en la figura 3.22, toma como argumento un valor booleano (itera), en función del cual se comporta de una manera o de otra. Si se llama con «falso» (false), se iteran los «objetos» que abstraen los cerramientos (que forman parte del conjunto Cerramientos), llamando a su función miembro Resuelve, la cual implementa una iteración del bucle interno de la figura 3.21 y retorna «verdadero» (true) si no se ha producido una modificación en la temperatura de las zonas. Este proceso se repite hasta que todos los cerramientos retornan true. De esta manera, los balances de energía de todos los cerramientos se van resolviendo simultáneamente, lo que mejora considerablemente la convergencia.

Para conseguir que se actualice la composición de los gases en el proceso de resolución, de acuerdo con lo indicado en $\S 3.4 .3$, debe llamarse a Resuelve con true. En este caso, en cada iteración del bucle do ... while, la función se llama a sí misma con false como 


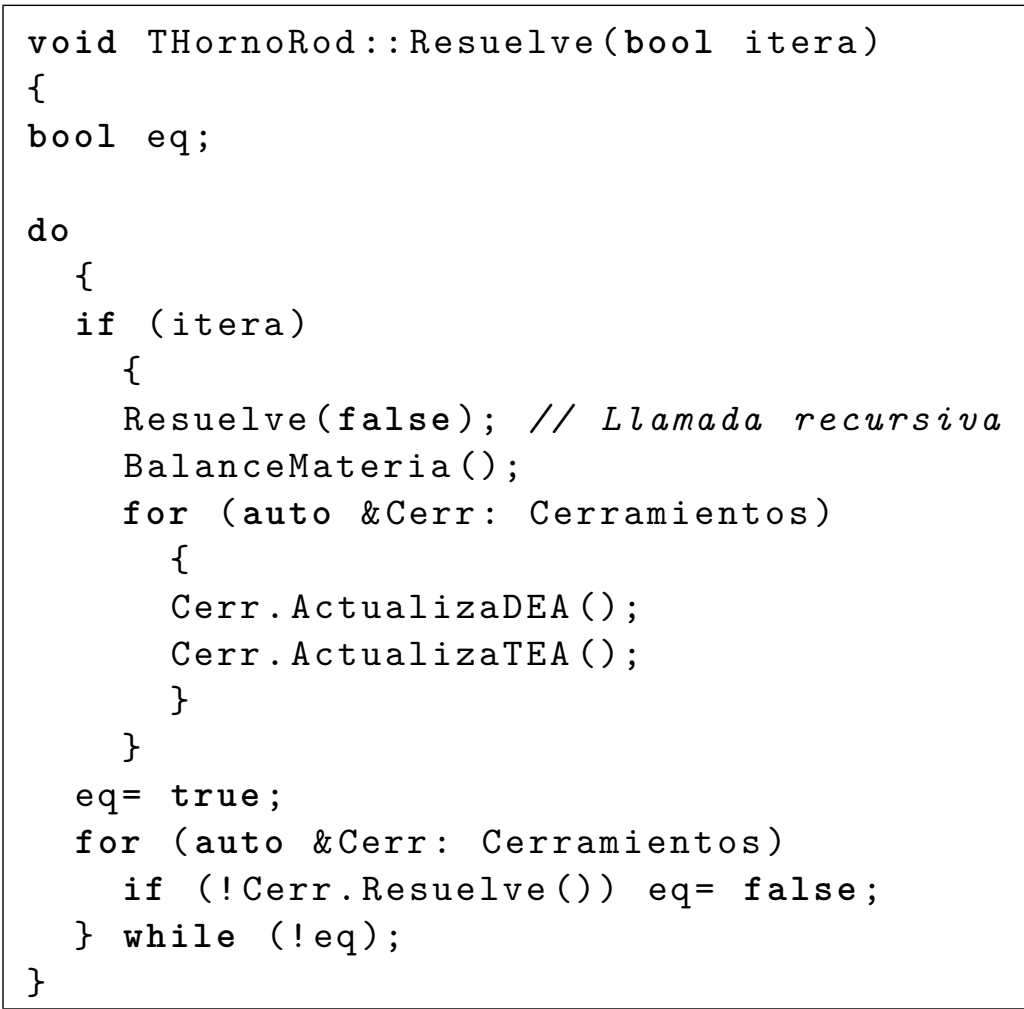

Figura 3.22. Código $\mathrm{C}++$ que implementa el algoritmo de resolución de los balances de energía.

argumento. Seguidamente, se actualizan los balances de materia y las DEA/TEA de todos los cerramientos, tras lo que se comprueba si la solución actual continua siendo válida, en cuyo caso, finaliza el bucle. Mediante el uso de llamadas recursivas se consiguen unificar los dos niveles de iteración presentados en la figura 3.21 en una única función. Esta función permite minimizar el número de DEA a calcular no sólo en el problema presentado en esta sección, sino también en el cálculo de la regulación del horno que se expone en §3.5.

Para obtener un error inferior al 0,01\% en las temperaturas de las zonas, habitualmente se requieren miles de iteraciones en el nivel interior y alrededor de diez iteraciones en el nivel exterior, lo que involucra la resolución de decenas de miles de sistemas de ecuaciones densos y tridiagonales, y la evaluación de unos pocos millones de DEA. Esto se traduce en un tiempo de cálculo inferior a un minuto en un ordenador personal modesto.

\subsection{Cálculo de la regulación del horno}

Plantear el problema de la manera recogida en $§ 3.4$ (las únicas incógnitas son las temperaturas de las distintas zonas) es de escasa utilidad práctica. El ciclo de cocción (caudal másico de baldosas y temperaturas de consigna para los gases) es una especificación del proceso, mientras que lo que es a priori desconocido e interesa optimizar es la regulación de quemadores; esto es, el caudal de gas natural a aportar en cada quemador. Se ha ideado un algoritmo muy simple que permite plantear el problema de manera más conveniente. 


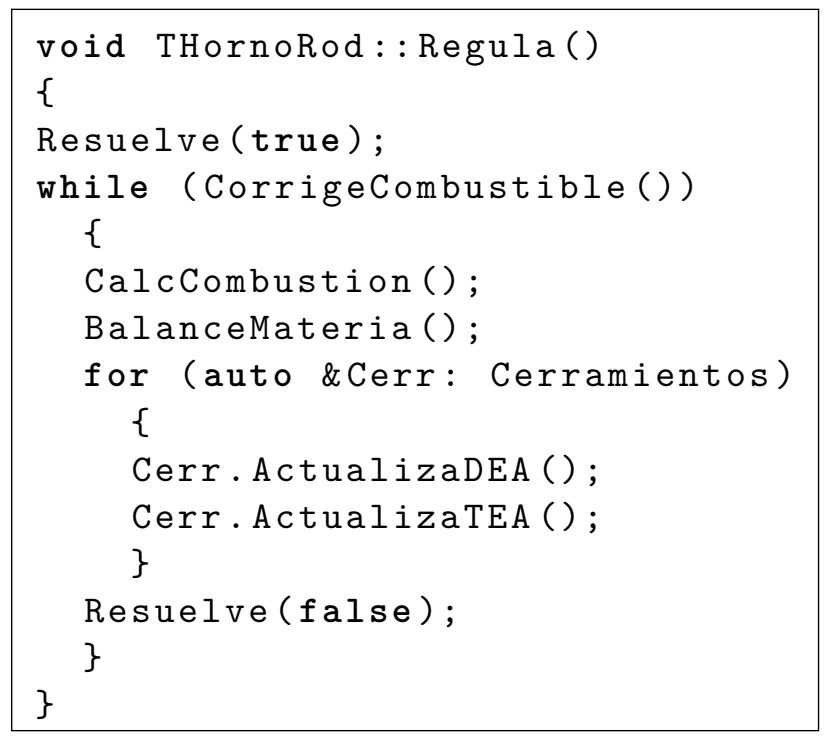

Figura 3.23. Código $\mathrm{C}++$ que implementa el algoritmo de regulación del horno.

Así pues, en esta sección, se considerará que se conocen las temperaturas de consigna que deben alcanzar los gases en los sectores en las que éstas se registran, en lugar de los caudales de combustible. Supóngase, sin embargo, que se parte de una regulación de caudales de combustible para la que las temperaturas de los gases son relativamente próximas a los valores de consigna. La idea básica del algoritmo consiste en suponer que el caudal adicional de combustible que hay que aportar (o sustraer) en cada sector $\Delta \dot{V}_{c}^{(i)}$ se invierte exclusivamente en elevar (o disminuir) la temperatura de los gases hasta el valor de consigna $T_{g \mathrm{SP}}^{(i)}$ :

$$
-\Delta_{r} H_{c}^{\ominus} \Delta \dot{V}_{c}^{(i)}=C^{(i)}\left(T_{g \mathrm{SP}}^{(i)}-T_{g, i}\right)
$$

siendo $C^{(i)}$ una «capacidad calorífica» adecuadamente escogida. Según lo indicado en $\S 3.3 .2 .1$ y $\$ 3.4$, parece razonable hacer:

$$
C^{(i)}=\sum_{k} \dot{V}_{k}^{(i)}\left(b_{1 k}+b_{2 k} \hat{T}_{g, i}\right)
$$

donde $k \in\left\{\mathrm{N}_{2}, \mathrm{O}_{2}, \mathrm{CO}_{2}, \mathrm{H}_{2} \mathrm{O}\right\}$.

Combinando las dos últimas expresiones, se obtiene la ecuación utilizada para la corrección de los caudales de combustible:

$$
\Delta \dot{V}_{c}^{(i)}=\alpha \frac{\sum_{k} \dot{V}_{k}^{(i)}\left(b_{1 k}+b_{2 k} \hat{T}_{g, i}\right)}{\Delta_{r} H_{c}^{\ominus}}\left(T_{g, i}-T_{g \mathrm{SP}}^{(i)}\right)
$$

en la que se ha introducido un factor de relajación $\alpha$ para mejorar la convergencia. El algoritmo de regulación consiste básicamente en corregir repetidamente los caudales de combustible de acuerdo con la ecuación (3.147) hasta que se alcancen las temperaturas de consigna. 


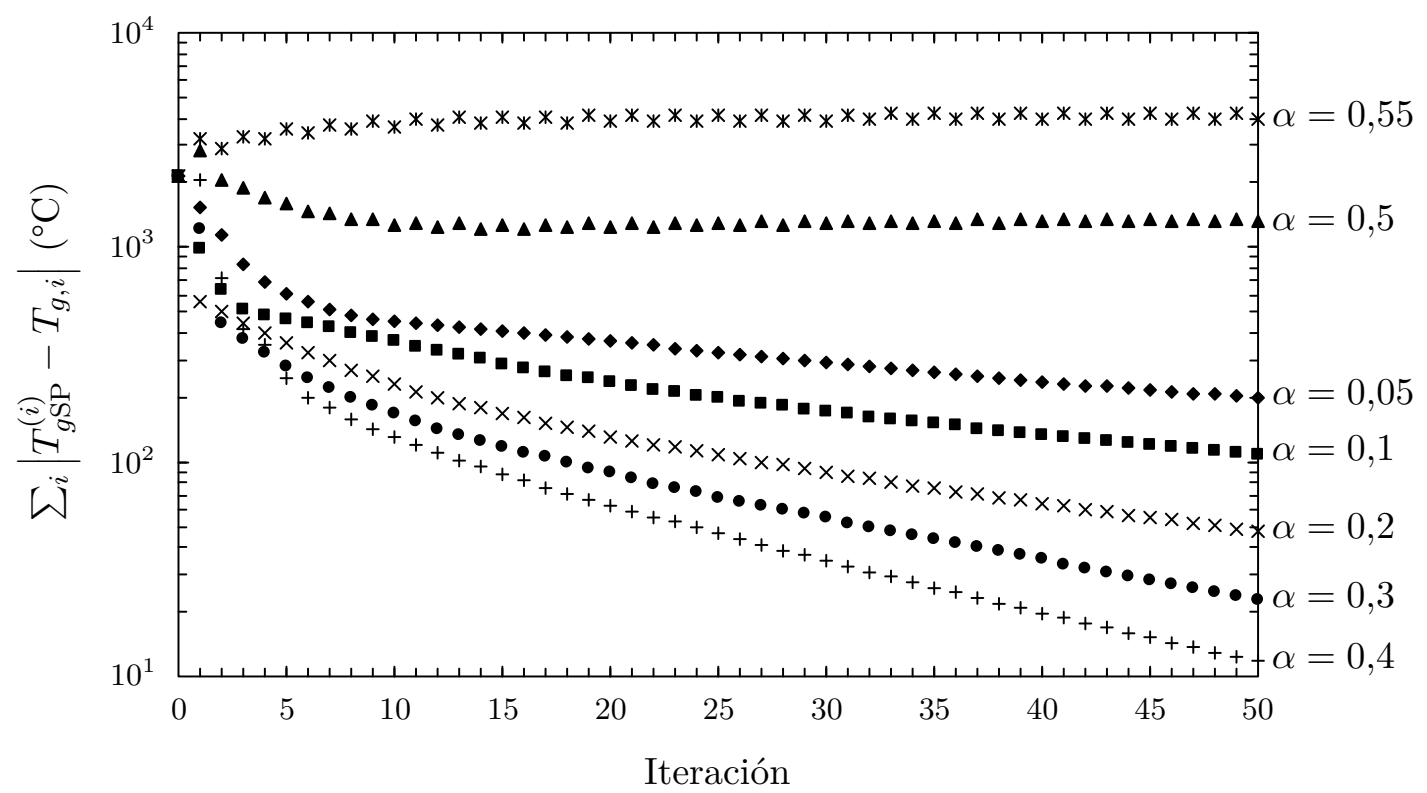

Figura 3.24. Convergencia del algoritmo de cálculo de la regulación del horno para distintos valores del factor de relajación.

Gracias a la función Resuelve, definida en la figura 3.22, el algoritmo de cálculo de la regulación del horno puede implementarse de una manera simple y eficiente (figura 3.23). En primer lugar se resuelven los balances de energía, exigiendo consistencia en la atmósfera del horno, para lo que ejecuta Resuelve(true). Tras este primer paso (que evitaría obtener una solución errónea en caso que el horno ya estuviera correctamente regulado), se inicia una secuencia en la que se corrigen los caudales de combustible (y comburente), se recalculan las DEA/TEA y se resuelven los balances de energía. Este proceso se interrumpe cuando ya no es necesaria ninguna corrección. La resolución de los balances de energía puede realizarse con Resuelve(false), ya que la progresión del algoritmo es lo suficientemente gradual como para asegurar la consistencia de la atmósfera del horno. Aún así, es evidente que este algoritmo es más costoso que el presentado en §3.4.

En la figura 3.24 se ofrece un ejemplo de la progresión del algoritmo. Lamentablemente, el coeficiente $\alpha$ es necesario para que se produzca la convergencia. No se puede especificar un valor de $\alpha$ que funcione adecuadamente de manera general, ya que depende del horno en particular considerado, pero el comportamiento de la figura 3.24 es bastante típico. Además, no es demasiado laborioso encontrar un valor óptimo de $\alpha$ por prueba y error.

Tras describir el funcionamiento básico del algoritmo, deben mencionarse ciertas características adicionales que éste debe reunir para que la solución obtenida sea realista. En este sentido, debe recordarse que los quemadores están agrupados en anillos (§1.4.1.2). A cada anillo le corresponde una temperatura de consigna, en función de la cual el sistema de control del horno actúa sobre todos quemadores del grupo. Así pues, las correcciones calculadas con la ecuación (3.147) deben distribuirse entre todos los quemadores del anillo.

Un aspecto que debe decidirse para el cálculo de la regulación del horno son los caudales de aire comburente que se aportarán para completar la combustión. Parece que pueden seguirse tres estrategias: 
Aire constante. Es el caso más simple. Sencillamente se especifican los caudales de aire iniciales, que se mantienen constantes durante el proceso iterativo.

Exceso de aire global constante. En este caso, se mantiene el exceso de aire considerando el horno en su conjunto (pero excluyendo el aire de enfriamiento). En cada iteración, los caudales de aire se corrigen mediante:

$$
\dot{V}_{a, k+1}^{(i)}=\dot{V}_{a, k}^{(i)} \frac{\sum_{(i)} \dot{V}_{c, k+1}^{(i)}}{\sum_{(i)} \dot{V}_{c, k}^{(i)}}
$$

en la que el índice $k$ se refiere a la iteración.

Exceso de aire individual constante. Es similar al anterior, pero en este caso se mantiene el exceso de aire en cada sector respecto a la situación inicial:

$$
\dot{V}_{a, k+1}^{(i)}=\dot{V}_{a, k}^{(i)} \frac{\dot{V}_{c, k+1}^{(i)}}{\dot{V}_{c, k}^{(i)}}
$$

Mediante estas estrategias pueden realizarse regulaciones del horno representativas de la práctica industrial. De manera similar, existen condiciones en las que el algoritmo es incapaz de encontrar ninguna solución, ya que dichas condiciones serían irrealizables en la práctica (por ejemplo, una curva de cocción con forma de «dientes de sierra»). 

The Romans supposedly tested each new bridge by requiring the design engineer to stand underneath while chariots drove over it.

- Ted L. Anderson [115]

\section{Validación del modelo}

\subsection{Experimental}

En este capítulo se evalúa la capacidad del modelo para realizar predicciones. A tal fin, se seleccionaron tres hornos en los que se efectuaron una serie de determinaciones experimentales, de manera que pudieron comparase los datos obtenidos experimentalmente con las predicciones del modelo.

Las dimensiones más relevantes de los hornos estudiados se resumen en la tabla 4.1. Los hornos 2 y 3 eran mucho más largos que el horno 1, por lo que, a igualdad de duración del ciclo de cocción, serían capaces de procesar una mayor producción. Los hornos 1 y 2 eran de la firma SACMI, mientras que el horno 3 era de la firma SITI. Cada fabricante de hornos tiene una manera propia de realizar la ingeniería de detalle del horno, pero el concepto general del horno siempre se ajusta a lo descrito en $§ 1.4$.

En la figura 4.1 se muestran esquemas de las partes de los hornos estudiados destinadas al calentamiento. Se identifican los módulos dotados de quemadores y cómo se encuentran los quemadores agrupados en anillos, a los cuales se les ha asignado una referencia. El horno 1 contaba con 112 quemadores, distribuidos en 12 anillos de 8 quemadores y 4 anillos de 4 quemadores. El horno 2 disponía de un total de 176 quemadores en 19 anillos de 8 quemadores y 6 anillos de 4 quemadores. El horno 3 tenía instalados 152 quemadores en 18 anillos de 8 quemadores y 2 anillos de 4 quemadores.

Los tres hornos contaban con una serie de cortafuegos. La hipótesis 8, adoptada para la formulación del modelo, consiste en que los cortafuegos cierran totalmente la sección de paso de los gases. En la realidad industrial pueden encontrarse desviaciones con respecto a esta situación ideal. Precisamente, al ejecutar los experimentos se constató que algunos cortafuegos estaban totalmente abiertos o solamente cerraban parte de la sección de paso. Se adoptó el criterio de considerar únicamente en los cálculos posteriores aquellos cortafuegos que cerraban más de la mitad de la sección de paso de los gases. La posición de

Número de

\begin{tabular}{cccccc} 
Horno & $\begin{array}{c}\text { Longitud total } \\
(\mathrm{m})\end{array}$ & $\begin{array}{c}\text { Anchura útil } \\
(\mathrm{m})\end{array}$ & $\begin{array}{c}\text { Número total } \\
\text { de módulos }\end{array}$ & $\begin{array}{c}\text { módulos en el } \\
\text { calentamiento }\end{array}$ & Fabricante \\
\hline 1 & 69 & 2,4 & 33 & 20 & SACMI \\
\hline 2 & 118 & 2,5 & 56 & 34 & SACMI \\
\hline 3 & 113 & 2,6 & 51 & 31 & SITI \\
\hline
\end{tabular}

Tabla 4.1. Características principales de los hornos estudiados. 


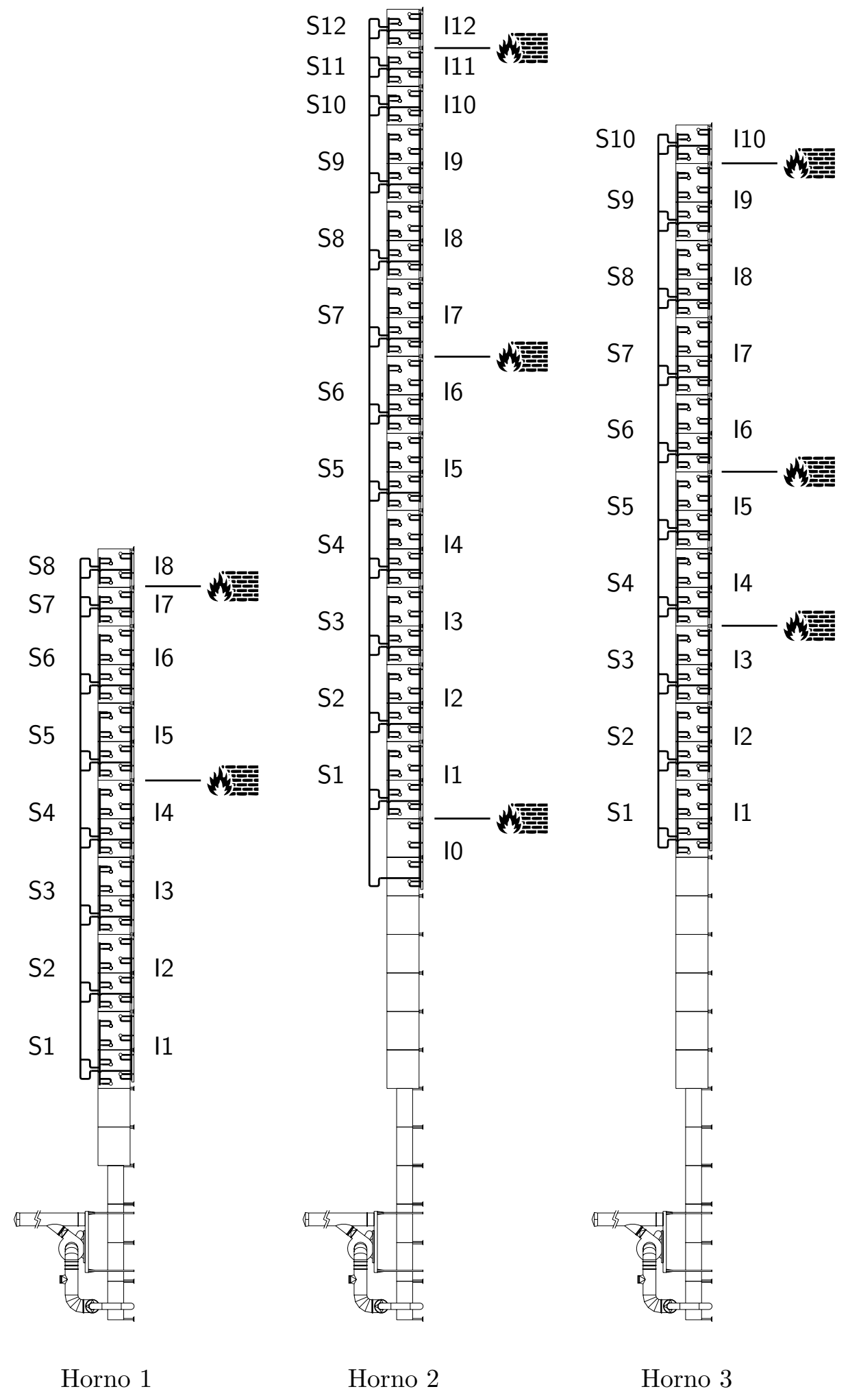

Figura 4.1. Esquema de la parte de calentamiento de los hornos estudiados. 


\begin{tabular}{cccccc} 
Horno & Producto & $\begin{array}{c}\text { Formato } \\
(\mathrm{cm} \times \mathrm{cm})\end{array}$ & $\begin{array}{c}\text { Espesor } \\
(\mathrm{mm})\end{array}$ & $\begin{array}{c}\text { Pérdidas por } \\
\text { calcinación }(\%)\end{array}$ & $\begin{array}{c}\text { Contenido en } \\
\mathrm{CaCO}_{3}(\%)\end{array}$ \\
\hline 1 & Azulejo & $35 \times 35$ & 8 & 9,7 & 12,5 \\
\hline 2 & Azulejo & $20 \times 50$ & 9 & 9,5 & 12,8 \\
\hline 3 & Porcelánico & $30 \times 60$ & 10 & 4,1 & 0,5 \\
\hline
\end{tabular}

Tabla 4.2. Productos fabricados en los hornos estudiados.

los cortafuegos que satisfacían esta condición se identifica mediante el símbolo en la figura 4.1. El resto de cortafuegos (uno o dos, según el horno) no se tuvieron en cuenta en los cálculos.

Para la realización de los experimentos, se tomó la precaución de escoger un periodo durante el cual se produjera un único producto de manera continuada, de modo que pudieran completarse las mediciones en unas condiciones estacionarias (hipótesis 1). Las características de los productos procesados en cada horno se recogen en la tabla 4.2.

En los hornos 1 y 2 los productos fabricados eran azulejos, mientras que en el horno 3 se producía porcelánico. Este hecho se refleja en el contenido en carbonatos de las composiciones procesadas (expresados como $\mathrm{CaCO}_{3}$ en la tabla 4.2). Las composiciones de azulejo se formulan para que tengan una proporción concreta de carbonatos, los cuales aportan características esenciales al producto: porosidad, estabilidad dimensional y fases cristalinas estables frente la humedad. Por el contrario, en las composiciones de porcelánico los carbonatos se consideran una impureza, pues reducen el intervalo de cocción y promueven la deformación de las piezas [116]. El mayor contenido en carbonatos en las composiciones de azulejo conlleva mayores pérdidas por calcinación, como consecuencia del $\mathrm{CO}_{2}$ liberado. La pérdida de masa restante se atribuyó al $\mathrm{H}_{2} \mathrm{O}$ asociado a la deshidroxilación de los minerales arcillosos. Partiendo de este supuesto, pudieron calcularse las fracciones másicas de los componentes de referencia de las reacciones químicas consideradas (§3.3.2.2).

En la tabla 4.3 se detallan una serie de magnitudes relativas a las condiciones de operación de los hornos estudiados. Se aprecia cómo el horno 1, a pesar de tratarse de un horno de menor longitud que los hornos 2 y 3 , procesaba una producción similar a la de los otros hornos. Esto es debido a la utilización de un ciclo de cocción más corto en este horno. El horno 3, en el que se efectuaba la cocción de porcelánico, operaba a una temperatura máxima de cocción mayor que la utilizada en los hornos en los que se producía azulejo.

Consumo

Producción Duración del Temperatura Exceso de energético

\begin{tabular}{cccccc} 
Horno & $\left(\mathrm{m}^{2}\right.$ día $\left.^{-1}\right)$ & ciclo $(\mathrm{min})$ & máxima $\left({ }^{\circ} \mathrm{C}\right)$ & aire $(\%)$ & $\left(\mathrm{MJ} \mathrm{m}^{-2}\right)$ \\
\hline 1 & 6200 & 38 & 1100 & 17 & 33 \\
\hline 2 & 5500 & 60 & 1125 & 35 & 32 \\
\hline 3 & 6900 & 51 & 1185 & 8 & 35 \\
\hline
\end{tabular}

Tabla 4.3. Condiciones de operación de los hornos estudiados. 


\begin{tabular}{cccc} 
& \multicolumn{3}{c}{ Resistencia térmica $\left(\mathrm{m}^{2} \mathrm{~K} \mathrm{~W}^{-1}\right)$} \\
\cline { 2 - 4 } & Aislamiento bajo & Aislamiento medio & Aislamiento alto \\
\hline Bóveda & 0,6 & 0,7 & 0,8 \\
\hline Paredes laterales y solera & 2 & 3 & 4 \\
\hline
\end{tabular}

Tabla 4.4. Resistencias térmicas de las paredes utilizadas en los cálculos.

Los hornos estudiados disponían de un contador de gas natural de desplazamiento positivo que permitió tomar lecturas del volumen de gas natural consumido por el horno durante los experimentos. El PCI del gas natural se obtuvo de los datos de suministro [117-119]. A partir de estos datos, se calculó el consumo energético por unidad de producto (tabla 4.3), que fue mayor en el horno 3 , lo que concuerda con los datos indicados en $§ 1.2$. En la tabla 4.3 también se indica el exceso de aire global, que cuantifica el volumen de aire aportado de más en los quemadores, con respecto al volumen de aire estequiométricamente necesario para la combustión completa. Este dato se calculó a partir de los caudales de aire comburente en los quemadores, cuya determinación se describe más adelante.

Las paredes de los hornos estudiados estaban constituidas por capas de ladrillos refractarios y fibras refractarias comunes. Se calculó la conductividad aparente nominal de dichas estructuras laminadas a partir de datos de la bibliografía [76, 105], comprobándose que los valores así obtenidos eran insuficientes para explicar las temperaturas exteriores de las paredes medidas experimentalmente. El deterioro de los refractarios o la existencia de puentes térmicos podrían justificar tales discrepancias. Se optó por definir tres grados de aislamiento (alto, medio y bajo), distinguiendo además entre la bóveda, que al encontrarse suspendida consiste en un aislamiento más ligero, y el resto de paredes. Basándose en esta clasificación, pudieron atribuirse resistencias térmicas efectivas a cada una de las categorías (tabla 4.4), las cuales se determinaron heurísticamente para el horno 1 y pudieron reutilizarse satisfactoriamente en los otros hornos estudiados.

Para poder validar del modelo, era necesario conocer los caudales volumétricos de combustible y comburente aportados a cada quemador, pero los hornos de rodillos no sólo no disponen de un registro en continuo de estos caudales, sino que ni siquiera tienen instalados caudalímetros. En su lugar, los quemadores cuentan con tomas que permiten medir la presión estática del gas natural y del aire comburente a la que éstos acceden al quemador. Aunque estas magnitudes están relacionadas con los caudales, la relación es complicada, lo que ha propiciado que en la práctica industrial la regulación del horno se base en los valores de presión estática en los quemadores, sin realizar la transformación a caudales. Este hecho tiene implicaciones muy importantes que se analizan en el capítulo 5.

La dependencia entre las presiones estáticas del gas natural y del aire en los quemadores y los respectivos caudales puede expresarse en forma de ábacos, como el mostrado en la figura 4.2. Estas curvas características dependen tanto del tipo de quemador como de la tobera de salida. Es habitual que todos quemadores del horno sean iguales pero que las toberas sean distintas en diferentes partes del horno.

Se midió la presión estática de gas natural y de aire comburente en todos los quemadores de los hornos estudiados con un micromanómetro. El caudal de aire depende de la temperatura a la que se introduce en el quemador (figura 4.2), la cual se determinó mediante un termopar 


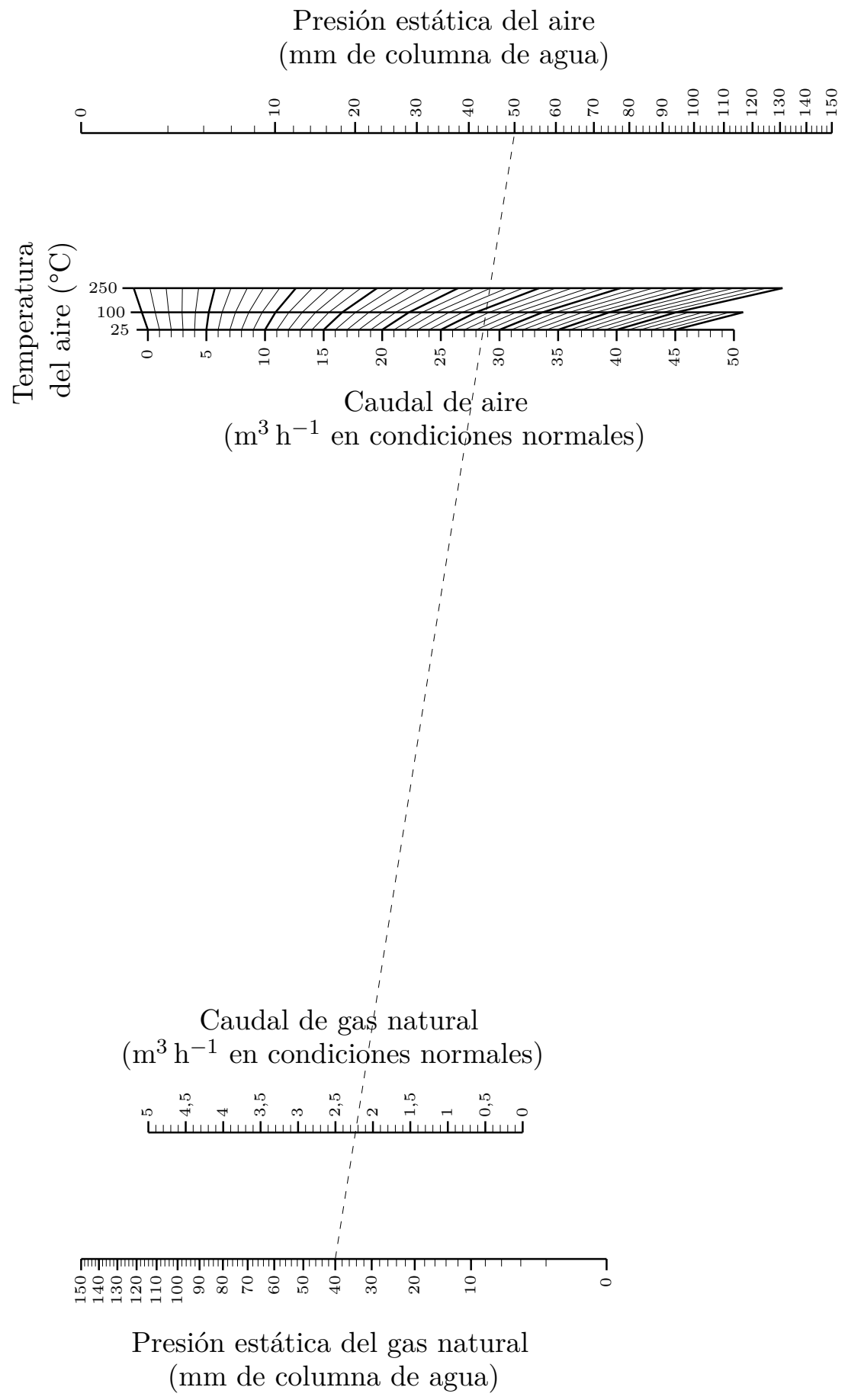

Figura 4.2. Ábaco para la determinación del caudal de combustible y comburente en el quemador. La línea discontinua es un ejemplo de uso. 
tipo J. A partir de los ábacos correspondientes, se calcularon los caudales volumétricos de gas natural y aire en cada quemador. Las diferencias entre la suma de los caudales de gas natural en cada quemador y el caudal total de gas leído en el contador del horno fueron inferiores al $10 \%$ en todos los casos. Se aplicó un factor de corrección a los caudales individuales de manera que su suma coincidiera exactamente con el caudal total registrado en el contador.

Los hornos de rodillos cuentan con una serie de mirillas, que consisten en orificios que atraviesan las paredes laterales del horno, permitiendo acceder a la atmósfera del horno, tanto por el canal superior como por el inferior. Las mirillas permitieron tomar muestras de los gases del horno en distintas posiciones a lo largo del mismo. Las muestras extraídas se analizaron con un analizador de gases, consistente en un sensor electroquímico de $\mathrm{O}_{2}$, pudiéndose determinar el contenido en $\mathrm{O}_{2}$ en los humos secos. Disponer de la evolución del contenido en $\mathrm{O}_{2}$ a lo largo del horno permitió, por una parte, comprobar la consistencia de los caudales de combustible y comburente determinados experimentalmente y, por otra parte, calcular la cantidad de aire de enfriamiento que se incorporaba al calentamiento.

La estimación del caudal de aire de enfriamiento incorporado al calentamiento se realizó mediante el método descrito en la referencia [16]. En este método, partiendo de los caudales de combustible y comburente determinados experimentalmente, se plantean los balances de materia descritos en §3.3.2.1, de manera que la fracción volumétrica de $\mathrm{O}_{2}$ en los humos en cualquier posición a lo largo del horno puede expresarse como una función del caudal de aire de enfriamiento. De este modo, se tienen tantas ecuaciones como puntos de medida, siendo la única incógnita el caudal de aire de enfriamiento que accede al calentamiento. Para resolver este sistema sobredeterminado, se recurre al método de los mínimos cuadrados.

La información indicada hasta el momento es suficiente para efectuar la simulación de los hornos estudiados, haciendo uso del algoritmo presentado en §3.4, para obtener como resultado la temperatura de todas las zonas en las que se divida el horno. La validación del modelo consistió en comparar dichas temperaturas con una serie de temperaturas determinadas experimentalmente. Esta es la manera convencional de efectuar este tipo de estudios [76].

Las mirillas de los hornos permitieron no sólo estudiar el contenido en $\mathrm{O}_{2}$ en los gases, sino también determinar su temperatura. Para ello, se introdujeron termopares tipo K o $\mathrm{S}$ (dependiendo de la temperatura) a través de las mirillas, pudiéndose determinar la temperatura de los gases en los canales superior e inferior a lo largo del horno.

Se determinó también la temperatura de los rodillos introduciendo un termopar en su interior. Se asumió que el valor así obtenido representaba la temperatura de la superficie exterior de los rodillos, lo que se basa en la consideración indicada anteriormente (§3.3.2.4), por la cual no se tienen pérdidas de energía por conducción a través de los rodillos. La validez de esta hipótesis se discute en base a los resultados obtenidos (§4.2).

Por último, se realizaron mediciones de temperatura de las paredes exteriores del horno. En lugar de tomar medidas puntuales, se tomó una termografía de las paredes laterales y la bóveda de cada módulo, de manera que, mediante posterior tratamiento por software de las termografías, pudieron obtenerse temperaturas medias representativas de cada módulo. Dicho análisis también permitió determinar la emisividad de estas superficies. 
(a)

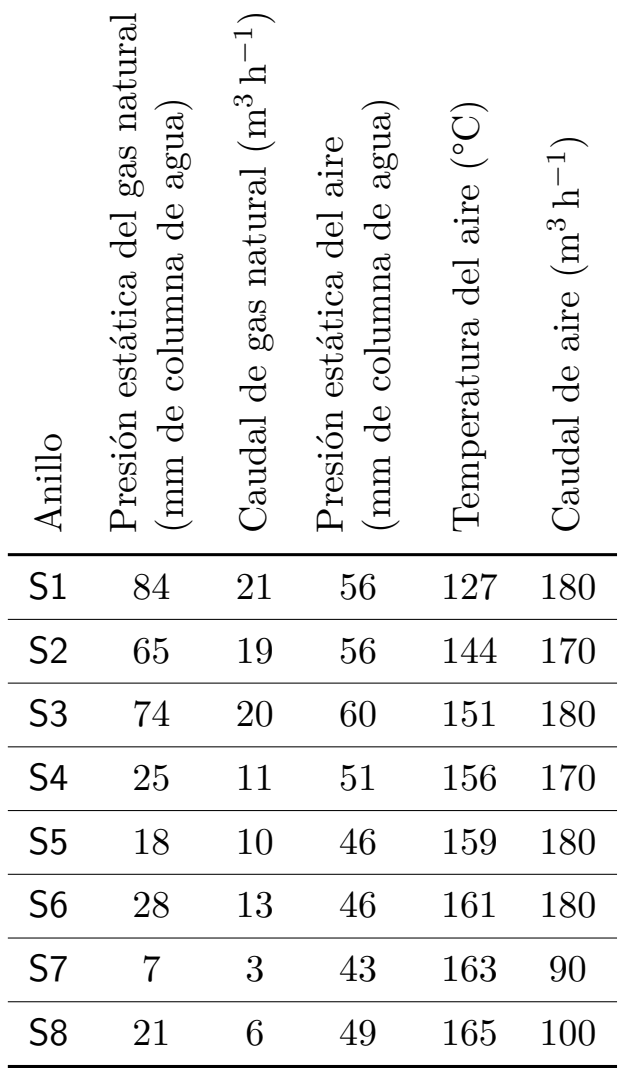

(b)

\begin{tabular}{|c|c|c|c|c|}
\hline 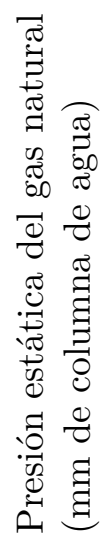 & 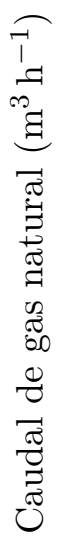 & 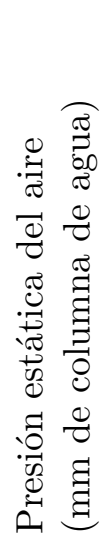 & 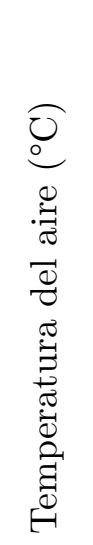 & 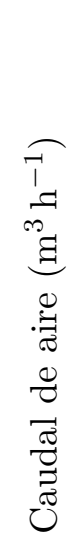 \\
\hline 89 & 22 & 48 & 137 & 180 \\
\hline 76 & 20 & 47 & 149 & 170 \\
\hline 67 & 19 & 50 & 156 & 180 \\
\hline 21 & 11 & 49 & 166 & 170 \\
\hline 22 & 12 & 47 & 159 & 190 \\
\hline 22 & 12 & 46 & 161 & 180 \\
\hline 40 & 8 & 48 & 162 & 90 \\
\hline 0 & 0 & 47 & 167 & 100 \\
\hline
\end{tabular}

Tabla 4.5. Regulación de quemadores del horno 1: (a) canal superior, (b) canal inferior.

\subsection{Resultados}

\subsubsection{Horno 1}

En la tabla 4.5 se detallan las condiciones de operación de los anillos de quemadores del horno 1 durante el transcurso de los experimentos. Se indican valores medios por anillo, lo cual es bastante apropiado, ya que se comprobó que las variables estudiadas eran aproximadamente constantes en todos los quemadores del anillo.

Los datos de la tabla 4.5 revelan que la regulación de quemadores en este horno estaba basada en la estrategia, muy extendida en el sector, de utilizar la misma presión estática de aire (la cual se regula manualmente) en todos los anillos del horno. Como consecuencia, el caudal de aire comburente aportado a lo largo del horno era relativamente constante (nótese que los anillos S7, S8, 17 e 18 tenían la mitad de quemadores; ver figura 4.1). No sucede lo mismo con los caudales de gas natural, establecidos por el sistema de control del horno, los cuales fueron sustancialmente diferentes en los distintos anillos. Esto significa que cada anillo de quemadores operaba en unas condiciones de exceso de aire distintas. Concretamente, en los 6 anillos más próximos a la entrada del horno (S1, S2, S3, I1, I2 e I3) se aportaba una cantidad de aire inferior a la estequiométrica, mientras que en el resto de anillos se aportaba aire en exceso; por ejemplo, el anillo S7 operaba con un $200 \%$ de exceso 
de aire. Los quemadores del anillo I8 estaban apagados suministrando aire para iniciar el enfriamiento de las piezas por su cara inferior.

El horno tenía instalado un intercambiador de calor en el enfriamiento rápido que permitía precalentar el aire comburente. La temperatura de entrada del aire a los quemadores se indica en la tabla 4.5. Se aprecia que, en el trasiego del aire hasta los quemadores, se producían pérdidas de energía significativas. En los anillos más próximos al intercambiador de calor se alcanzaban los $160^{\circ} \mathrm{C}$, pero en los anillos más alejados la temperatura del aire se había reducido unos $30^{\circ} \mathrm{C}$.

En la figura 4.3 se muestran las curvas de contenido en $\mathrm{O}_{2}$ en los gases a lo largo del horno. La línea continua representa los valores teóricos, calculados a partir de los datos de la tabla 4.5 y el caudal de aire de enfriamiento ajustado por mínimos cuadrados. Por el corolario 7.2, las curvas para los canales superior e inferior se trataron independientemente. Los valores obtenidos para el caudal de aire de enfriamiento fueron $150 \mathrm{~m}^{3} \mathrm{~h}^{-1}$ para el canal superior y $180 \mathrm{~m}^{3} \mathrm{~h}^{-1}$ para el canal inferior. Con este único parámetro de ajuste, se obtuvo un buen acuerdo entre los valores experimentales y los calculados. Además, se comprueba que la evolución del contenido en $\mathrm{O}_{2}$ en los canales superior e inferior es independiente; esto es, la curva de $\mathrm{O}_{2}$ no aporta indicios de que exista mezcla de gases entre ambos canales, lo que respalda la validez del corolario 7.2.

Se realizó la simulación del horno bajo las condiciones de operación estudiadas. En primer lugar, se efectuaron cálculos preliminares con el fin de decidir la longitud apropiada de los sectores en los que discretizar al horno (§3.1.2). Se comprobó que se obtenían resultados similares empleando sectores con una longitud igual a la de los módulos o utilizando sectores de medio módulo. Los resultados presentados corresponden a la segunda opción.

En la figura 4.4 se muestran las temperaturas de consigna para los gases (que coinciden con las registradas por los termopares del horno), las temperaturas medidas experimentalmente y las calculadas a partir del modelo. No todos los módulos del horno disponían de mirillas, por lo que no pudieron determinarse la temperaturas de los gases en los 10 primeros metros del horno.

El modelo fue capaz de predecir las temperaturas de los gases con una precisión mejor del $10 \%$. Dado que se trata de valores genuinamente teóricos, sin ningún parámetro de ajuste, este resultado se considera altamente satisfactorio. Las mayores discrepancias se encontraron en el canal inferior, en posiciones en torno a los $20 \mathrm{~m}$ de la entrada, donde la diferencias entre los valores experimentales y los calculados alcanzaron unos $60^{\circ} \mathrm{C}$. Curiosamente, en estas posiciones también se detectaron diferencias entre las temperaturas registradas por los termopares del horno y las medidas a través de las mirillas, siendo los resultados del cálculo más próximos a los valores registrados por el horno.

La figura 4.5 muestra las temperaturas medidas en el interior de los rodillos. Como se ha señalado, se consideró que dichas temperaturas eran similares a las de la superficie exterior de los rodillos, argumentando que las pérdidas de calor por conducción a través de los rodillos eran despreciables. Si se cumple esta condición, la temperatura de las superficies interior y exterior de los rodillos, así como la del aire contenido en ellos, debe coincidir. Si no es el caso, en la zona de máxima temperatura, donde la temperatura de los diversos elementos está más igualada, deberían detectarse diferencias de temperatura importantes entre los gases y el interior de rodillos, indicando que existe un flujo de calor a su través. Los resultados experimentales no aportaron evidencias de que se diera esta situación. 

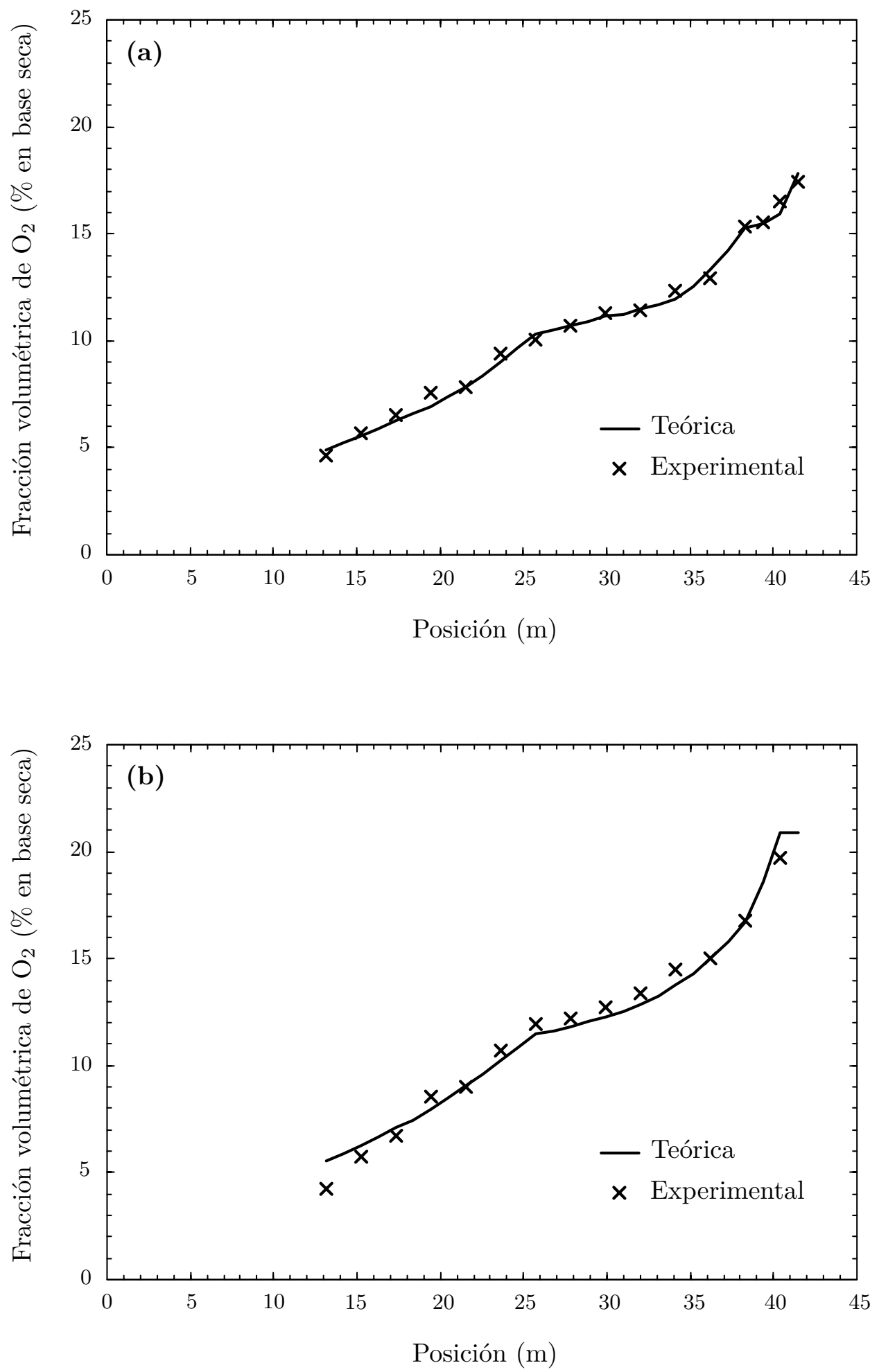

Figura 4.3. Fracción volumétrica de $\mathrm{O}_{2}$ en los gases del horno 1: (a) canal superior, (b) canal inferior. 

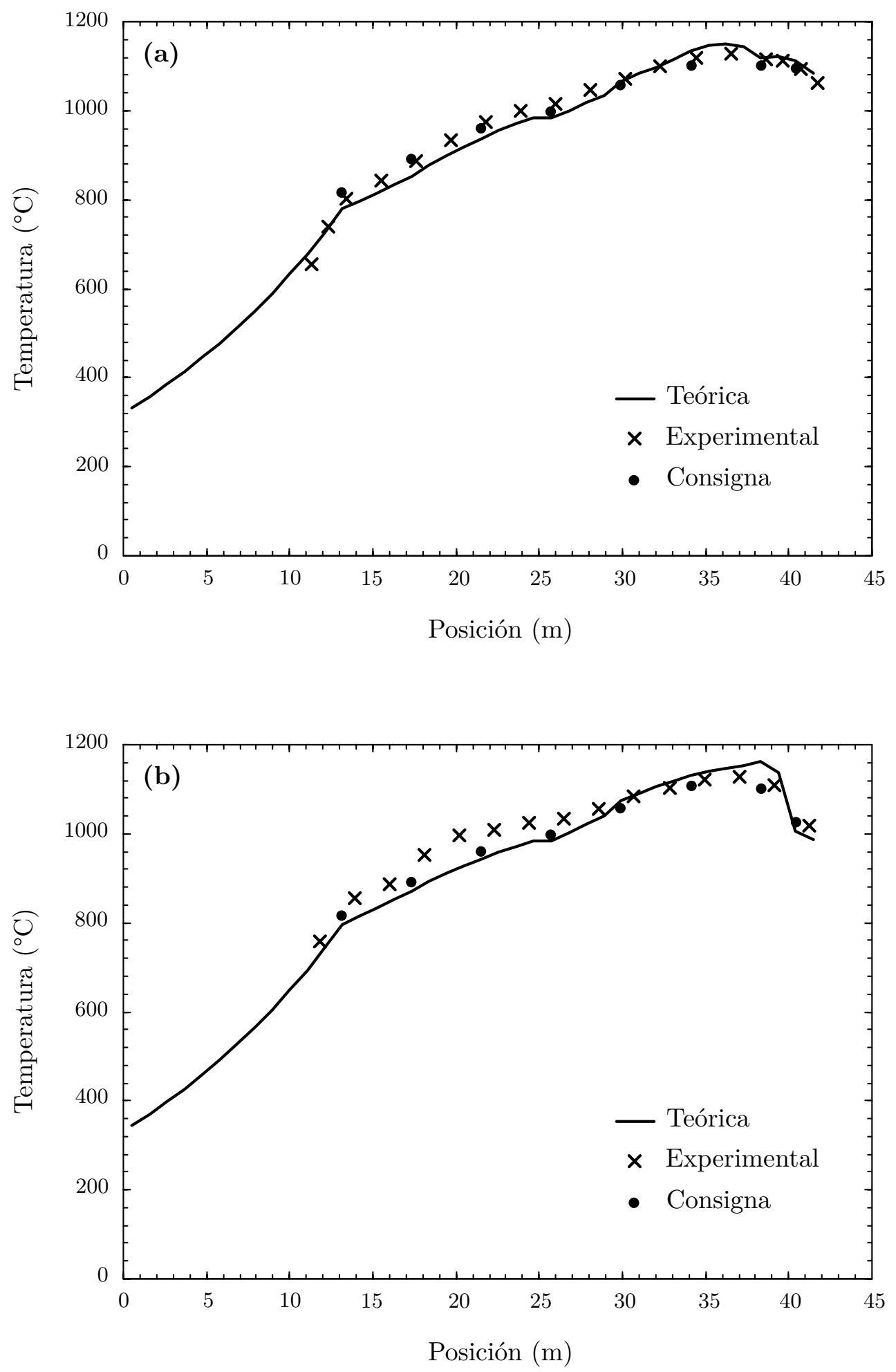

Figura 4.4. Temperatura de los gases del horno 1: (a) canal superior, (b) canal inferior. 


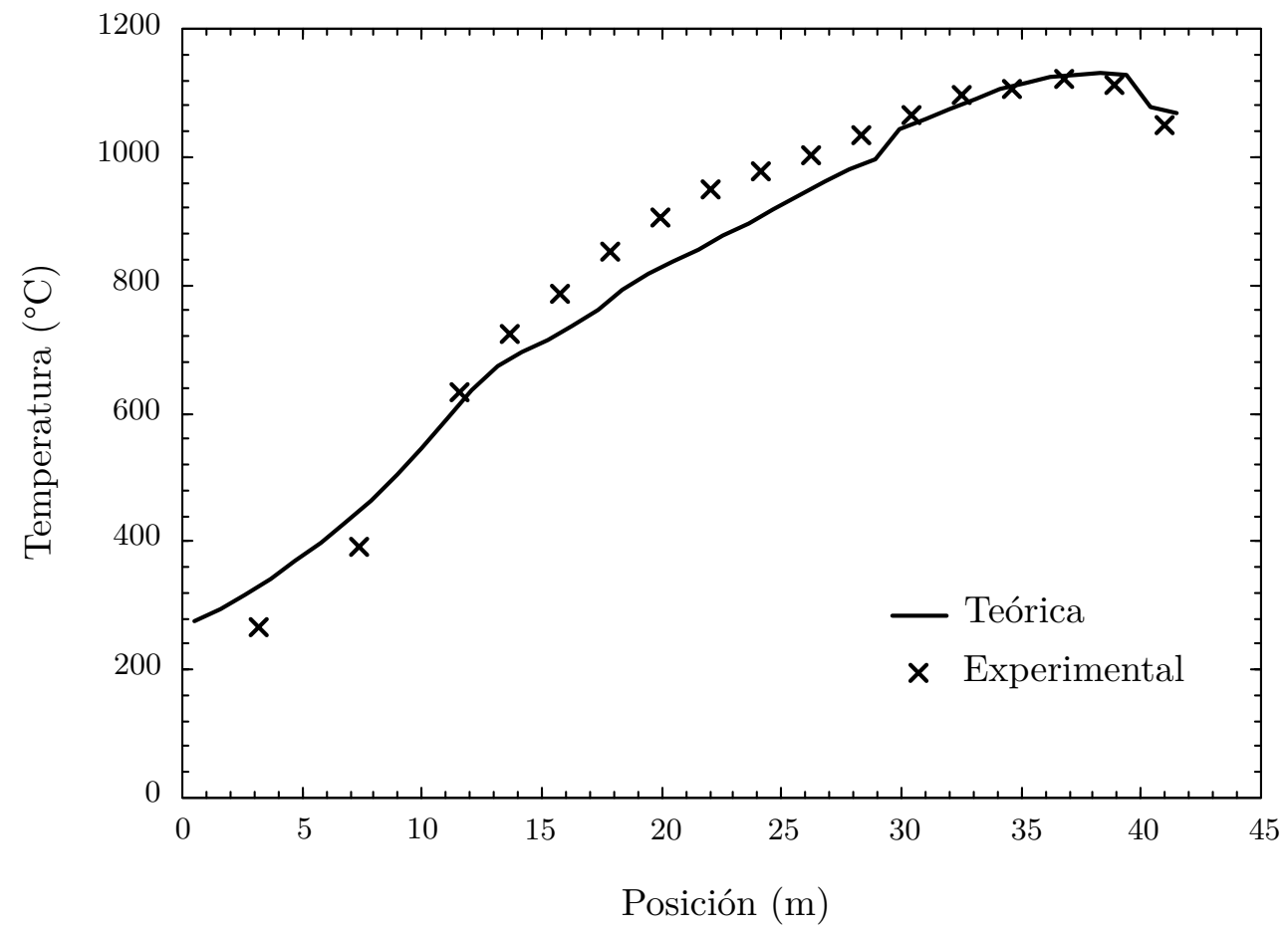

Figura 4.5. Temperatura de los rodillos del horno 1.

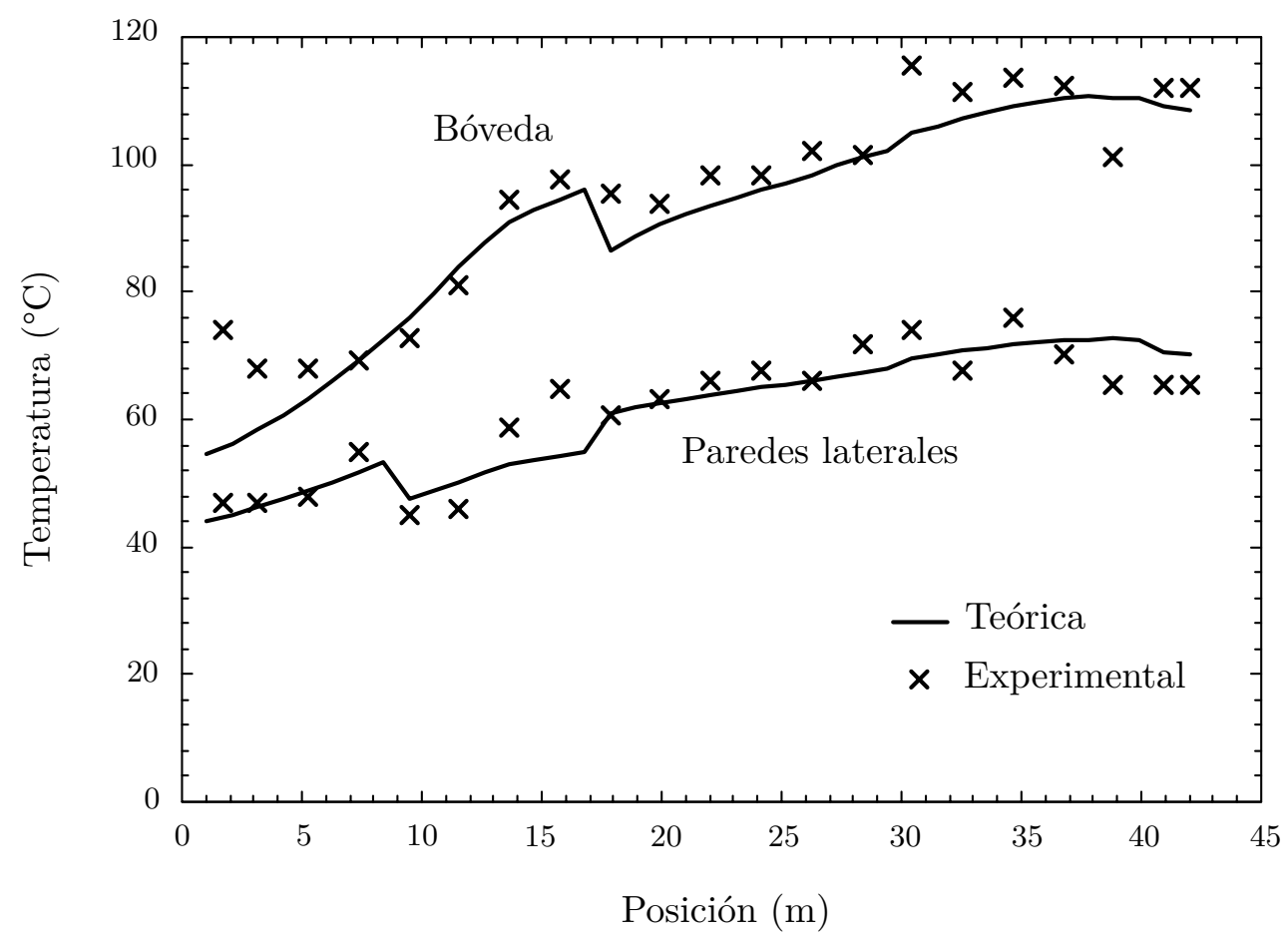

Figura 4.6. Temperatura exterior de las paredes del horno 1. 
La concordancia entre las temperaturas calculadas para los rodillos y los valores experimentales fue satisfactoria, aunque se obtuvieron diferencias de hasta $75^{\circ} \mathrm{C}$, precisamente en la zona donde se observaron mayores diferencias entre las temperaturas de los gases experimentales y teóricas. Posiblemente ambos hechos estén relacionados, aunque se desconoce la causa de los mismos.

Las temperaturas obtenidas para las superficies exteriores de las paredes del horno se muestran en la figura 4.6. Se aprecia que la superficie exterior de la bóveda estaba más caliente que la de las paredes laterales, lo que se atribuye a un menor aislamiento de la bóveda. El planteamiento sencillo de utilizar resistencias térmicas efectivas (tabla 4.4) fue suficiente para predecir la temperatura exterior de las paredes del horno y, por tanto, las pérdidas de energía asociadas.

\subsubsection{Horno 2}

La tabla 4.6 recoge la regulación de quemadores del horno 2. Los anillos I0 e I1 no estaban operando y, por ese motivo, no se incluyen en dicha tabla. Como en el caso del horno 1, la estrategia de regulación del aire comburente estaba, en líneas generales, basada en utilizar una presión estática del aire constante que, en este caso, se situaba en torno a los $20 \mathrm{~mm}$ de columna de agua. Sin embargo, en una serie de anillos (S1, S10 e I5), la presión estática del aire se había ajustado atendiendo a las aportaciones de gas natural en dichos anillos.

El horno 2 también contaba con un sistema de precalentamiento del aire comburente, consiguiéndose alcanzar hasta $230^{\circ} \mathrm{C}$ en algunos anillos. Al igual que en el horno 1 , la temperatura del aire comburente disminuía con la distancia al intercambiador de calor. La caída de temperatura fue mayor en los anillos del canal superior, a los que el aire se les suministraba a través de una conducción situada en la bóveda, que en los del canal inferior, para los que la correspondiente conducción de aire transcurría por debajo de la solera. Las pérdidas de energía en el trayecto suponían un $1 \%$ de la energía aportada al horno mediante la combustión del gas natural.

En la figura 4.7 se muestran las curvas de contenido en $\mathrm{O}_{2}$ en los humos obtenidas en el horno 2. Los valores experimentales pudieron reproducirse satisfactoriamente mediante los datos de la tabla 4.6 y el caudal volumétrico de aire de enfriamiento ajustado por mínimos cuadrados, el cual tomó un valor de $200 \mathrm{~m}^{3} \mathrm{~h}^{-1}$, tanto por el canal superior como por el inferior.

En la figura 4.8 se presentan las temperaturas de los gases experimentales y calculadas. El modelo fue capaz de predecir las temperaturas de los gases con una exactitud incluso mejor que la obtenida para el horno 1. Las mayores diferencias se dieron puntualmente en el canal superior, en posiciones en torno a $25 \mathrm{~m}$ y $50 \mathrm{~m}$ desde la entrada del horno, donde alcanzaron hasta unos $50^{\circ} \mathrm{C}$. En el resto de posiciones, las diferencias de temperatura fueron del orden de las que se observaron entre las temperaturas registradas por el horno y las medidas a través de las mirillas. El acuerdo entre los valores experimentales y los teóricos en el canal inferior fue excelente.

Cabe mencionar que la temperatura calculada de salida de los gases fue sustancialmente inferior a la obtenida para el horno 1. Esta diferencia se interpreta que es debida a la utilización de un ciclo de cocción más largo en el horno 2 , lo que permite aprovechar mejor el contenido energético de los gases (véase §5.2.3). 


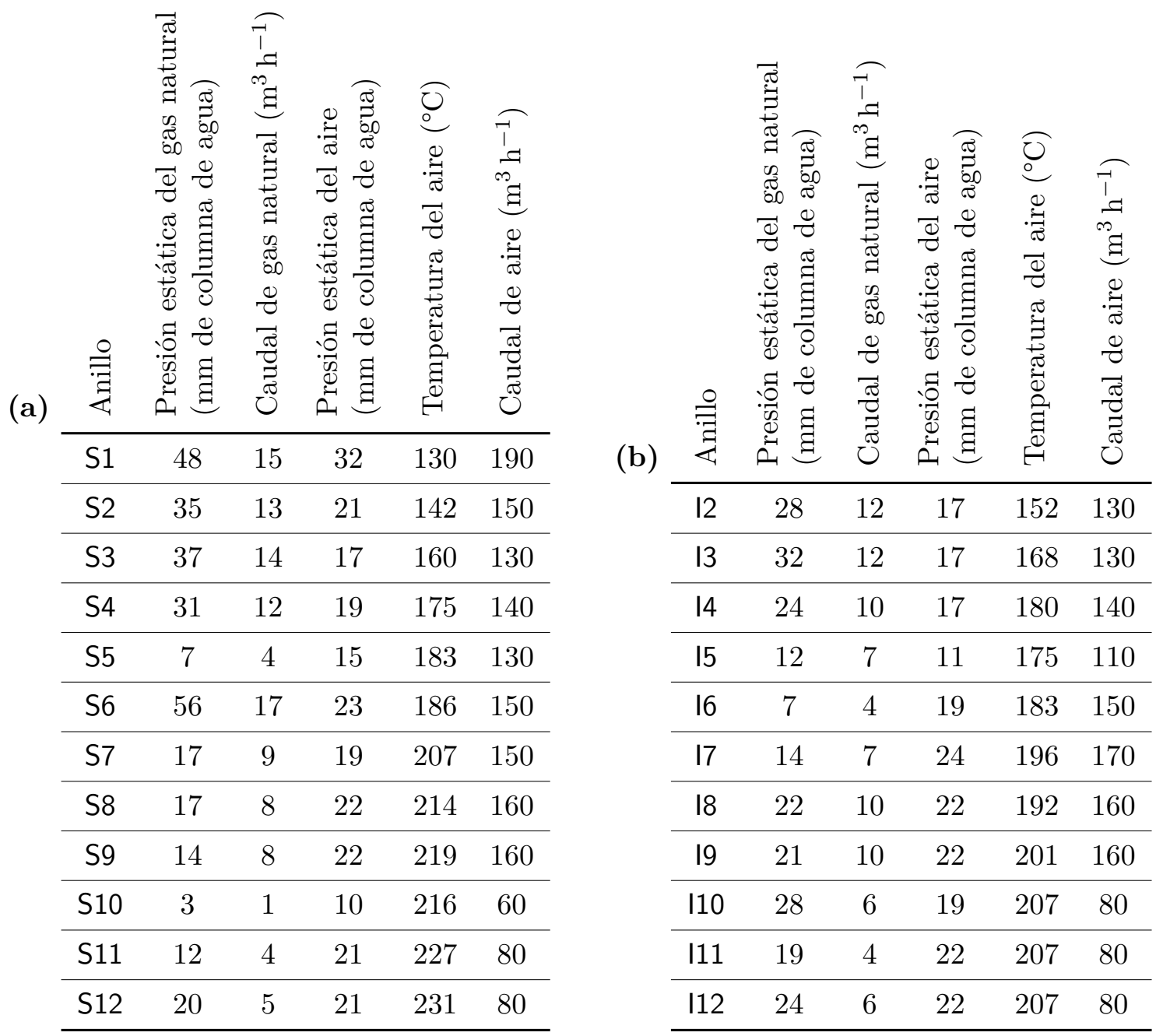

Tabla 4.6. Regulación de quemadores del horno 2: (a) canal superior, (b) canal inferior.

Las temperaturas de los rodillos experimentales y teóricas se comparan en la figura 4.12. El modelo predijo correctamente la evolución de las temperaturas de los rodillos, con un error máximo de aproximadamente $30^{\circ} \mathrm{C}$. No se observó la presencia de ningún error sistemático de la envergadura de los observados en el caso del horno 1. Este resultado contribuye a despejar posibles dudas acerca de los balances de energía para los rodillos que hubieran podido surgir atendiendo a los resultados del horno 1.

La figura 4.10 muestra las temperaturas exteriores de las paredes del horno 2. Las resistencias térmicas seleccionadas (tabla 4.4) permitieron calcular unas temperaturas exteriores de las paredes similares a las determinadas experimentalmente. Se cometió un cierto error (inferior a $10^{\circ} \mathrm{C}$ ) en la simulación de la temperatura exterior de las paredes laterales en la zona de máxima temperatura, que hubiera podido subsanarse utilizando resistencias térmicas específicas para esta zona. Por simplicidad, se prefirió utilizar las mismas resistencias térmicas para todos los hornos estudiados, ya que, en cualquier caso, no hubiera afectado significativamente a las temperaturas de los elementos interiores del horno. 

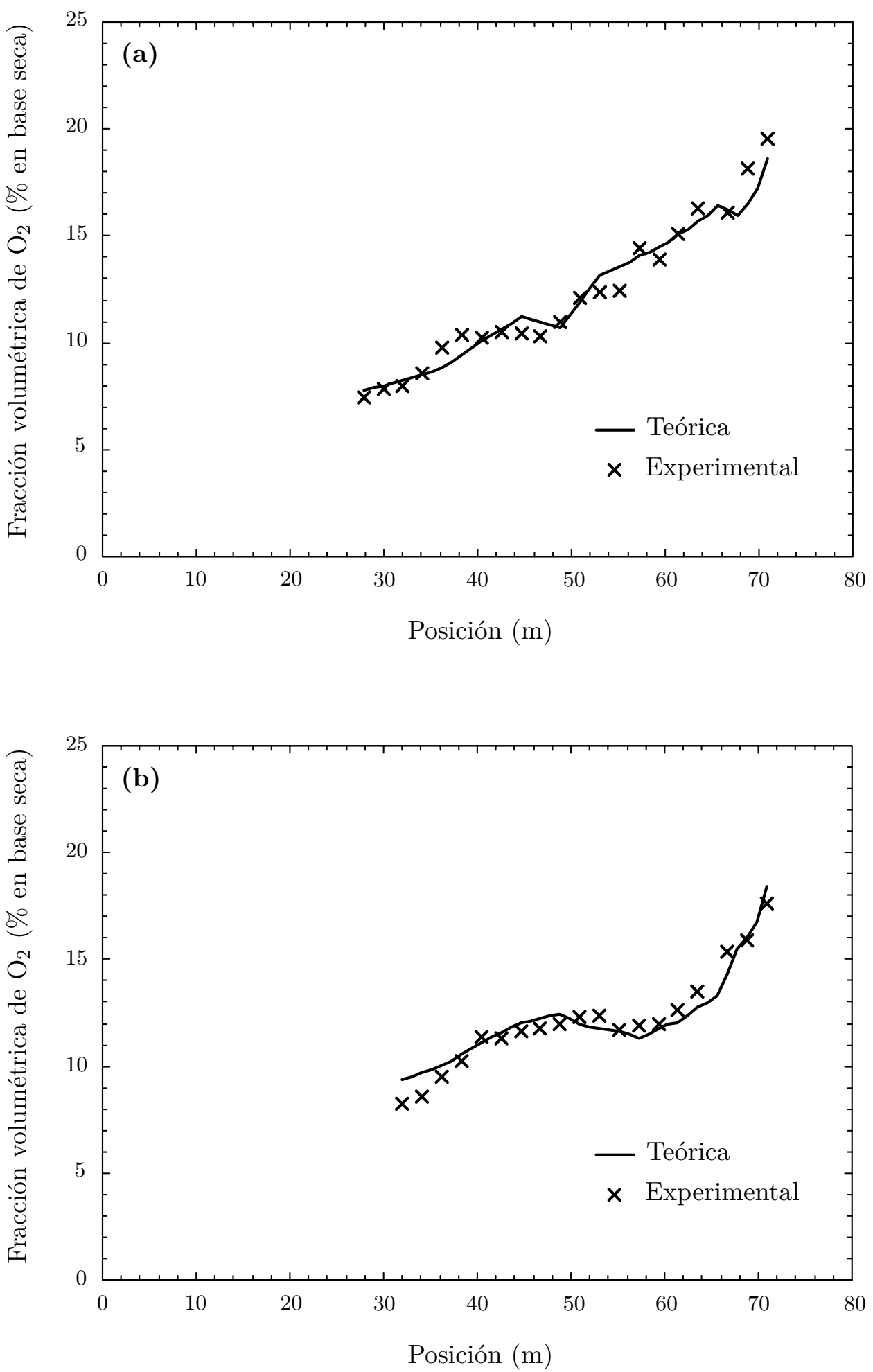

Figura 4.7. Fracción volumétrica de $\mathrm{O}_{2}$ en los gases del horno 2: (a) canal superior, (b) canal inferior. 

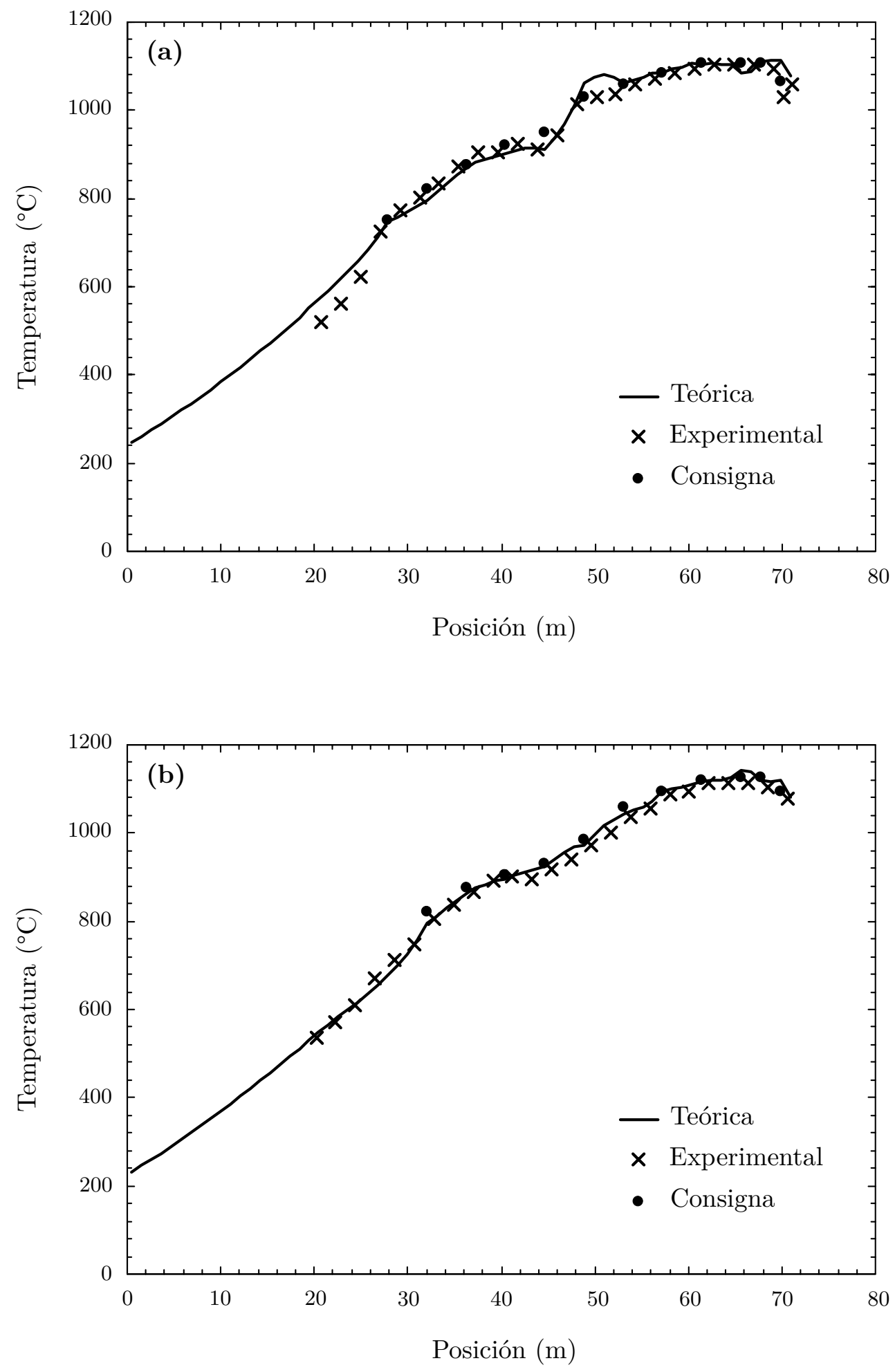

Figura 4.8. Temperatura de los gases del horno 2: (a) canal superior, (b) canal inferior. 


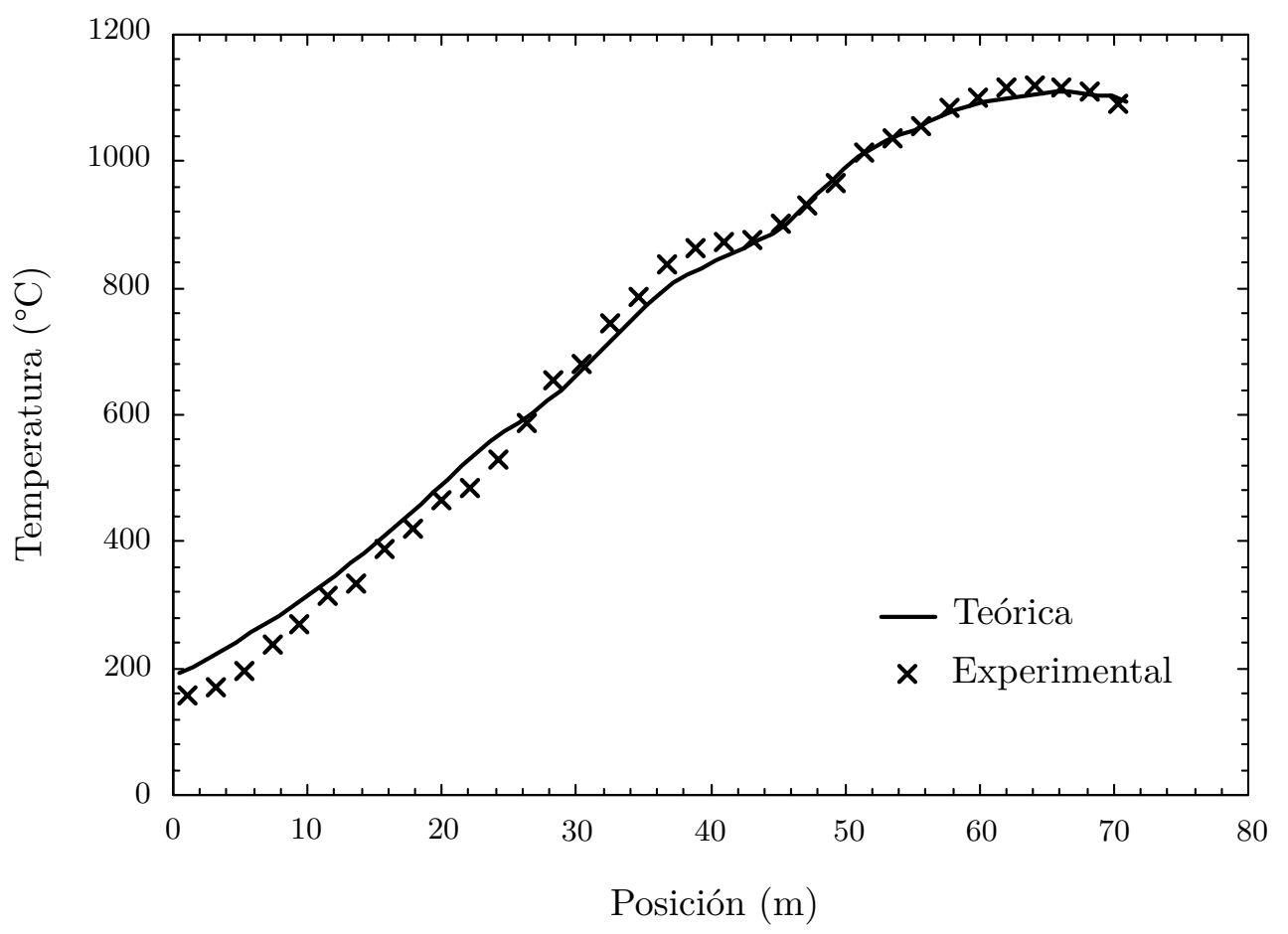

Figura 4.9. Temperatura de los rodillos del horno 2 .

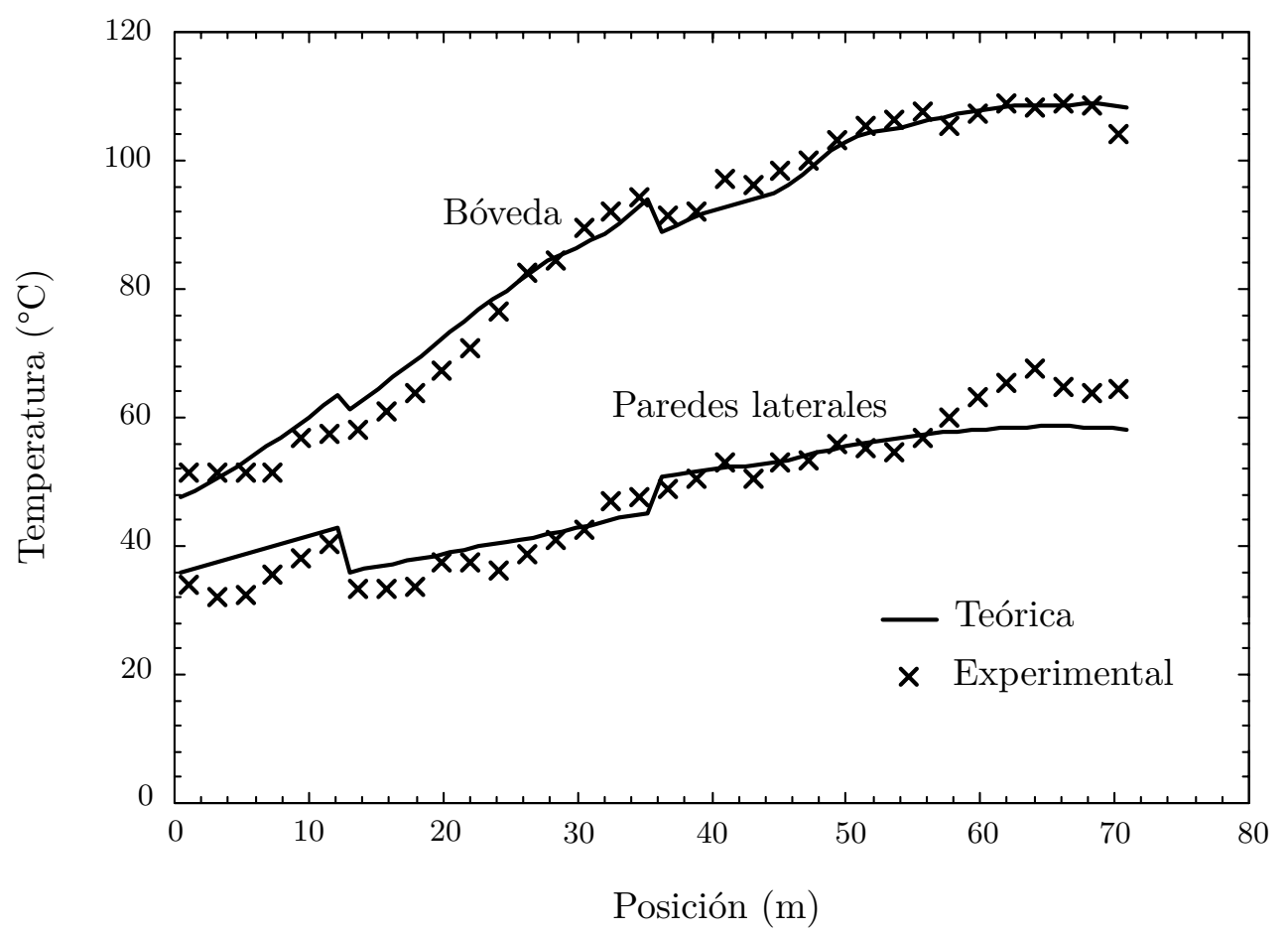

Figura 4.10. Temperatura exterior de las paredes del horno 2 . 


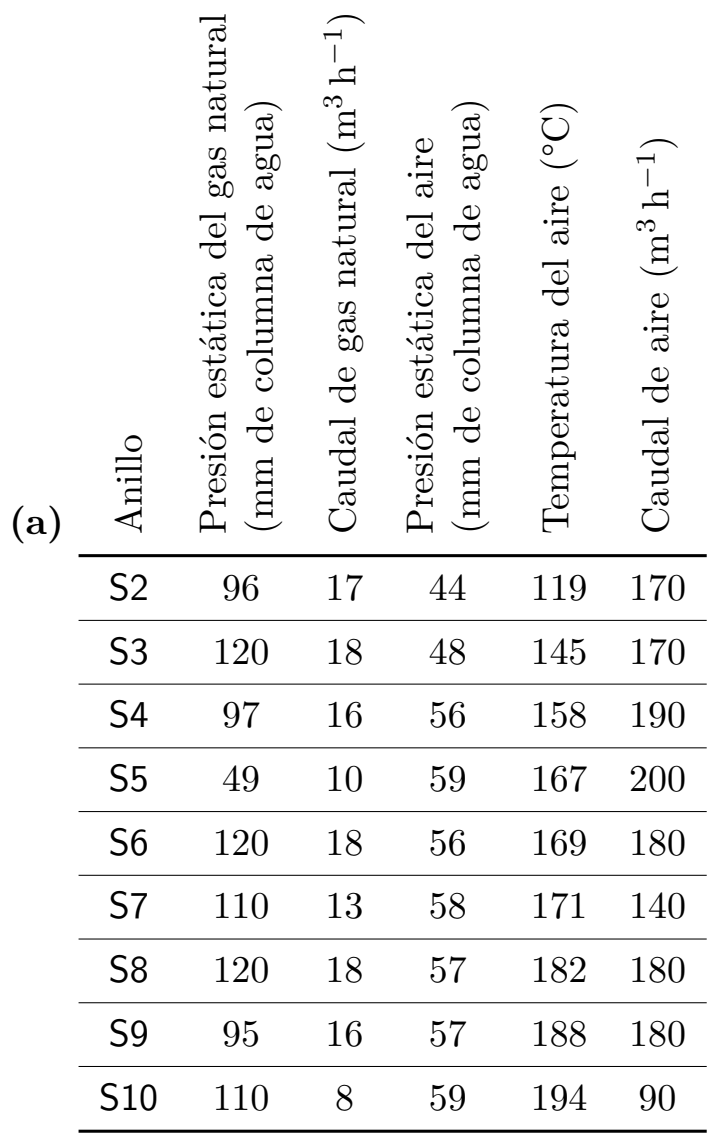

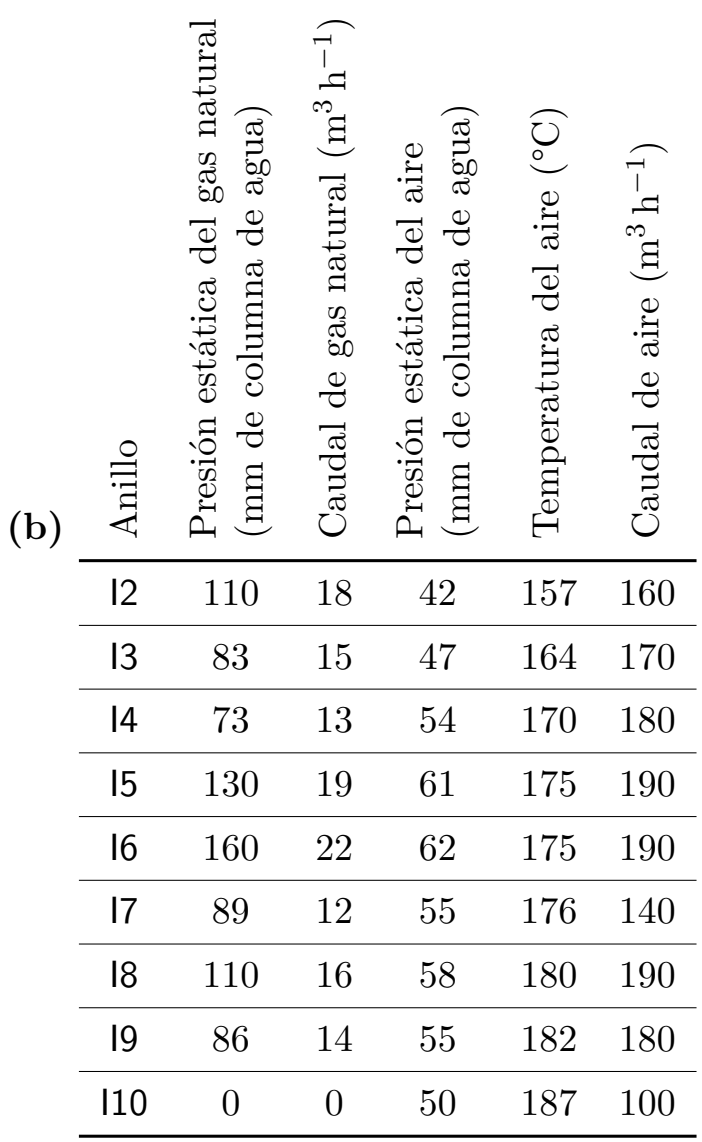

Tabla 4.7. Regulación de quemadores del horno 3: (a) canal superior, (b) canal inferior.

\subsubsection{Horno 3}

Las condiciones de operación de los anillos de quemadores del horno 3 se detallan en la tabla 4.7. Las conclusiones que pueden extraerse de estos datos son análogas a las indicadas para los hornos 1 y 2. Se empleaba prácticamente la misma presión estática del aire comburente en todos los anillos del horno, aunque, en este caso, se tenía la peculiaridad de que en los anillos S7 e 17 algunos quemadores no estaban operando.

No se consiguió obtener una lectura estable del contenido en $\mathrm{O}_{2}$ en los humos durante el transcurso de los experimentos. Se observó una falta de reproducibilidad en los valores obtenidos que no pudo atribuirse a interrupciones en la alimentación de baldosas o a otra causa de no estacionariedad. Se sospecha que los problemas experimentados eran de carácter instrumental. El principal inconveniente de no disponer de estos datos es que no pudo calcularse el caudal de aire de enfriamiento mediante el método utilizado para los otros dos hornos. En esta ocasión, tuvo que estimarse este valor, por lo que, basándose en los valores obtenidos para los otros hornos, se utilizó un valor de $200 \mathrm{~m}^{3} \mathrm{~h}^{-1}$ para ambos canales.

Nuevamente, se obtuvo un buen acuerdo entre los valores experimentales y calculados para las temperaturas de los gases (figura 4.11). Lo mismo puede decirse de la temperatura de los 

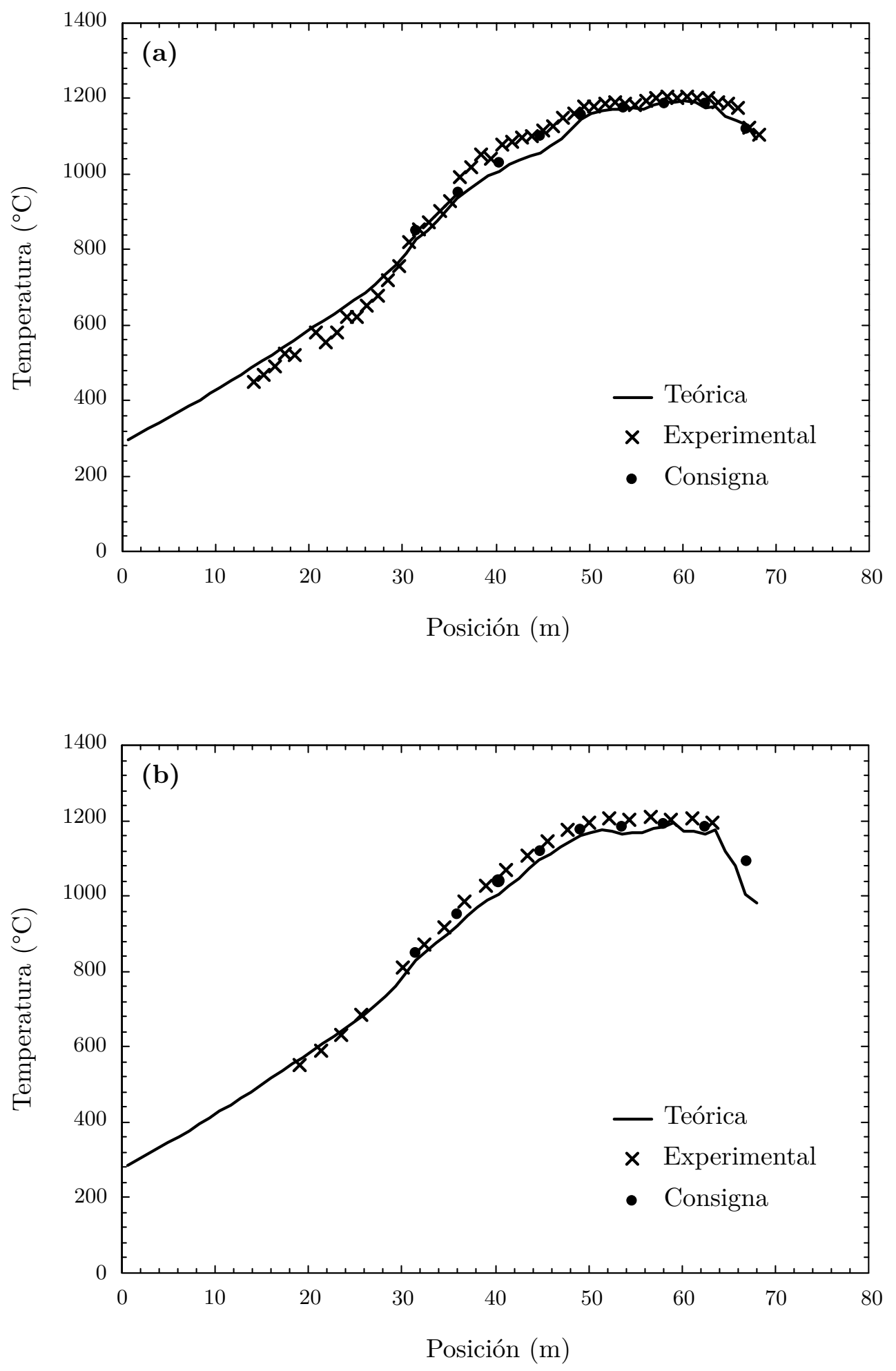

Figura 4.11. Temperatura de los gases del horno 3: (a) canal superior, (b) canal inferior. 


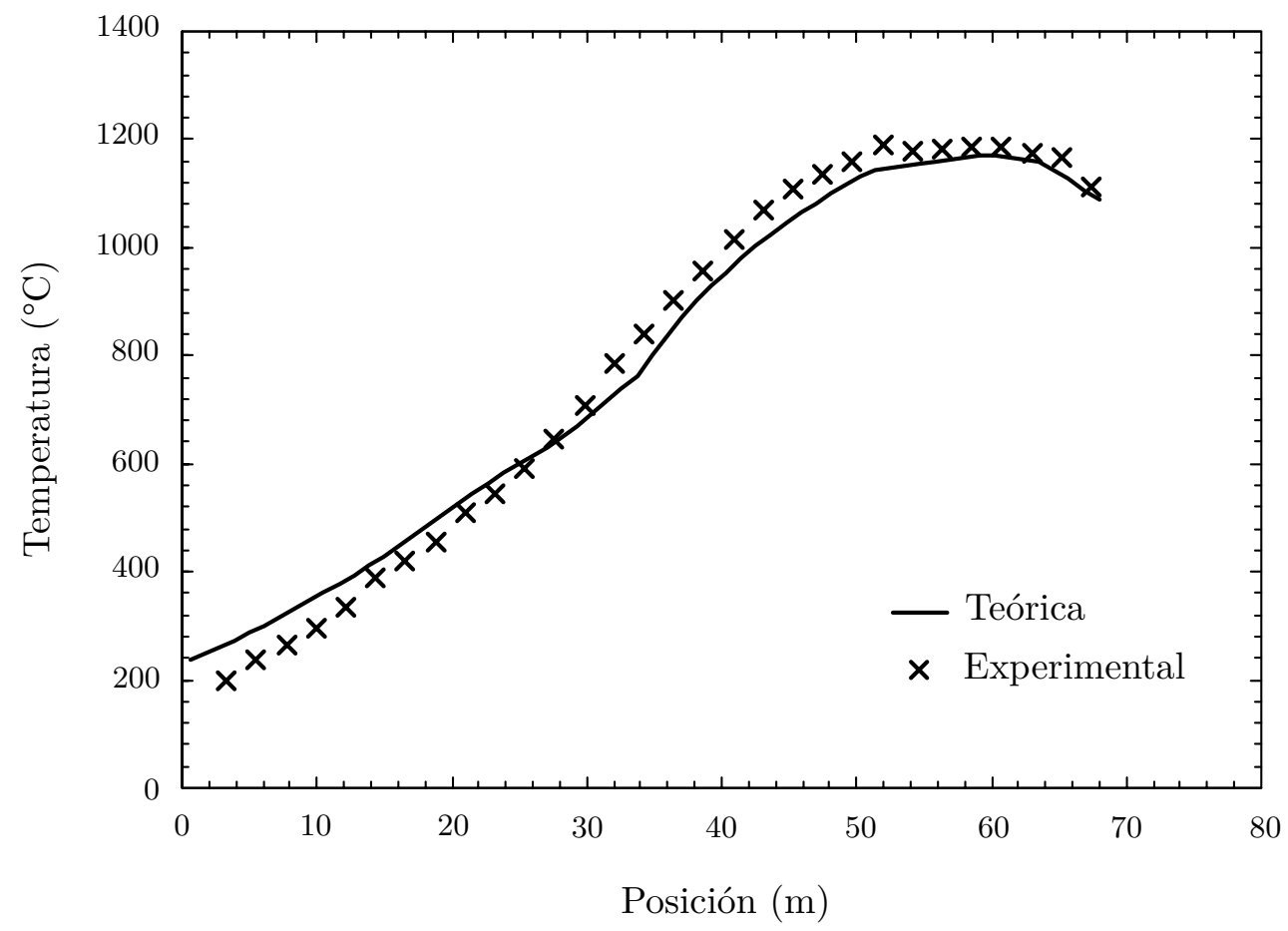

Figura 4.12. Temperatura de los rodillos del horno 3.

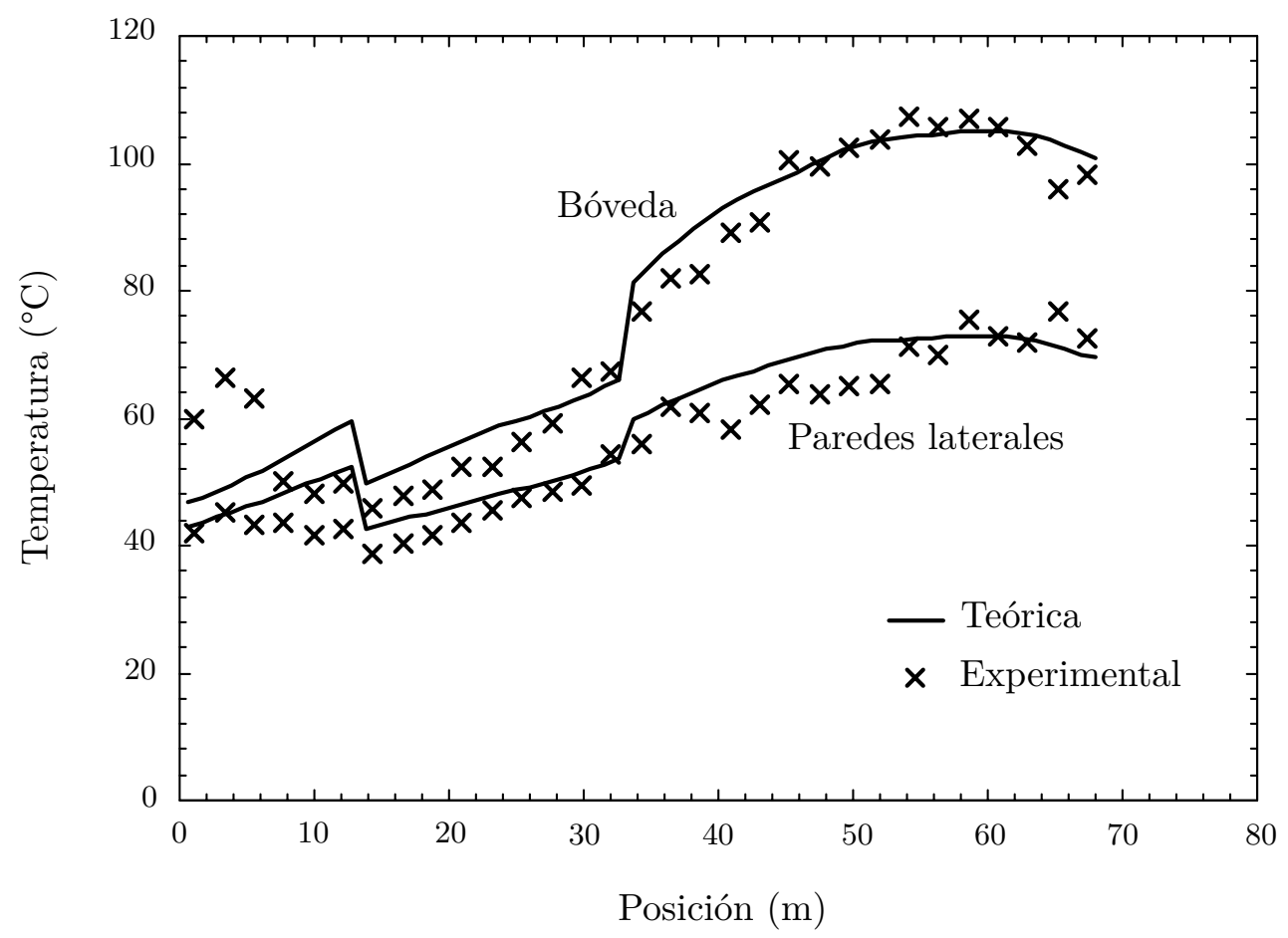

Figura 4.13. Temperatura exterior de las paredes del horno 3. 
rodillos, la cual se muestra en la figura 4.12. En ambos casos se subestimaron ligeramente las temperaturas máximas alcanzadas, pero se consiguió reproducir la tendencia de las temperaturas con una gran fidelidad.

Como en los casos anteriores, las temperaturas exteriores de las paredes pudieron simularse aceptablemente bien mediante las resistencias térmicas indicadas en la tabla 4.4. En este caso, tuvo que utilizarse una secuencia de aislamientos para la bóveda (bajo-alto-medio), diferente de la utilizada en los hornos 1 y 2 (bajo-medio-alto), para reproducir los resultados experimentales.

Los resultados presentados en este capítulo otorgan una gran confianza en la capacidad del modelo para simular la transferencia de energía en el horno de rodillos. En el capítulo siguiente se explota esta capacidad para realizar predicciones acerca de la influencia de ciertas variables de proceso sobre las condiciones de operación del horno. 
Forecasting is the art of saying what will happen, and then explaining why it didn't!

- Anónimo

\section{Aplicación del modelo}

\subsection{Caso de estudio}

En este capítulo se utiliza el modelo desarrollado para efectuar una serie de predicciones, principalmente relacionadas con el consumo energético del horno. Las situaciones estudiadas se corresponden con las recomendaciones habituales para el ahorro energético en la cocción de baldosas cerámicas en hornos de rodillos $[25,120]$.

Podría parecer contraproducente aplicar el modelo para simular una serie de medidas «conocidas». No obstante, es apropiado hacer una revisión de dichas acciones ya que, en ocasiones, el ahorro energético asociado ni siquiera se ha cuantificado. Asimismo, los efectos para los que se dispone de datos experimentales no son inmediatamente comparables con otros efectos obtenidos en escenarios distintos. Además, como se argumentará, es posible que, en algunos resultados experimentales, ciertos efectos estén confundidos. En definitiva, se considera conveniente dotar de un trasfondo teórico sólido a las acciones propuestas para el ahorro energético, así como ofrecer una valoración del peso relativo de cada efecto.

Como caso de estudio, se seleccionó un horno de rodillos hipotético de 50 módulos, 30 de ellos en el calentamiento (figura 5.1). El horno está dotado de anillos de quemadores que se extienden desde el 13 er $^{\text {er }}$ ódulo hasta el 30. ${ }^{\circ}$ Los anillos de los módulos $13 .^{\circ}$ a $28 .^{\circ}$ agrupan los quemadores del canal superior o inferior de dos módulos, mientras que los módulos 29. y $30 .^{\circ}$ disponían de anillos específicos.

La anchura útil del horno era de 2,5 m. El resto de características geométricas del horno, así como las propiedades de los materiales con los que estaba construido, estuvieron basadas en las de los hornos estudiados en el capítulo 4.

Se definió una curva de cocción base sobre la que realizar las modificaciones correspondientes, cuya forma se muestra en la figura 5.2. Se definieron dos parámetros característicos de dicha curva: la duración total del ciclo $(\tau)$ y la temperatura máxima de cocción $\left(T_{\text {máx }}\right)$. El resto de características de la curva de cocción se indican gráficamente en la figura 5.2, como por ejemplo la existencia de una etapa de descomposición, con una velocidad de calentamiento distinta, entre $800^{\circ} \mathrm{C}$ y $950{ }^{\circ} \mathrm{C}$. Al igual que en la situación industrial, la curva de cocción se especifica únicamente en posiciones concretas, a través de una serie de temperaturas de consigna para cada anillo de quemadores. Por simplicidad, se supuso que las temperaturas de consigna de los anillos superiores e inferiores coincidían.

La composición seleccionada era de azulejo, con un $13 \%$ en peso de carbonatos (en forma de $\left.\mathrm{CaCO}_{3}\right)$ y un $10 \%$ de pérdidas por calcinación, que se supusieron que eran debidas exclusivamente a la liberación de $\mathrm{CO}_{2}$ y $\mathrm{H}_{2} \mathrm{O}$ en las reacciones identificadas en §3.3.2.2.

Las variables estudiadas se recogen en la tabla 5.1. Como se ha indicado, la optimización de dichas variables constituye un conjunto de buenas prácticas para el ahorro energético en la cocción de baldosas cerámicas. Se definieron una serie de «valores de referencia» para 


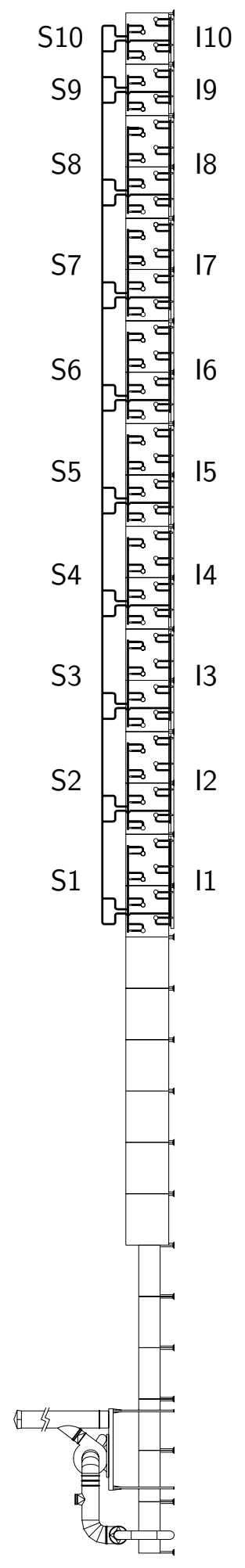

Figura 5.1. Esquema de la parte de calentamiento del horno estudiado. 


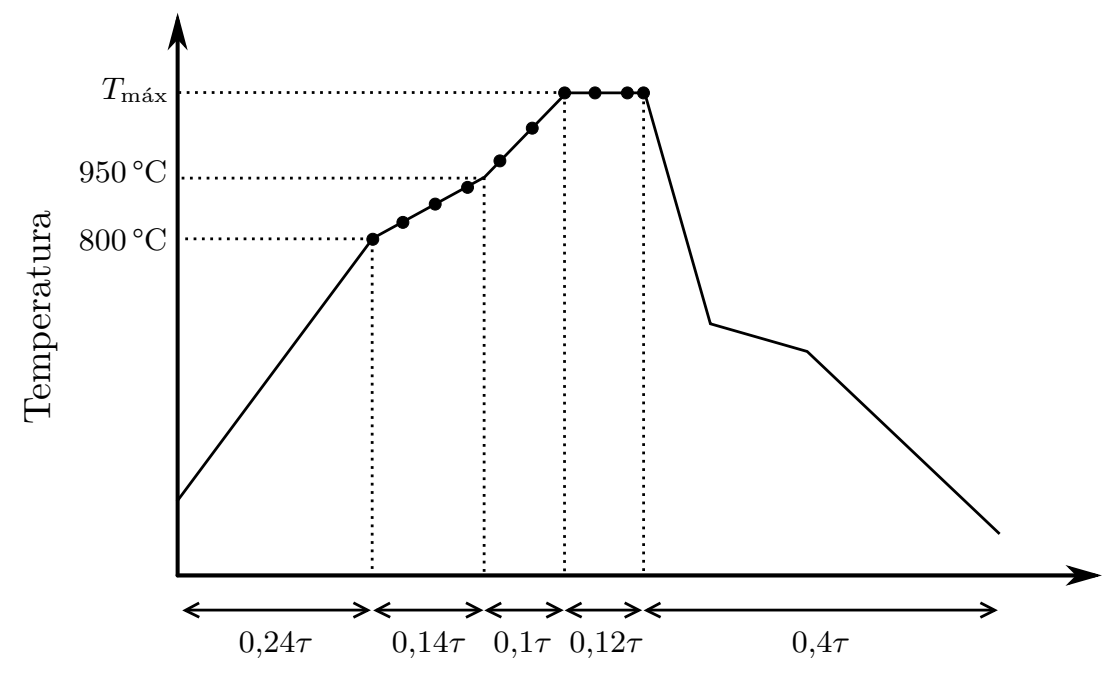

Tiempo

Figura 5.2. Curva de cocción. Las temperaturas de consigna se identifican mediante círculos.

las variables estudiadas, los cuales se detallan en la tabla 5.1. Se entiende que, cuando se modifique una determinada variable, el resto toma los valores de referencia.

Como en la práctica industrial, se utilizó la misma presión estática del aire de combustión en todos los anillos de quemadores del horno. Las modificaciones de las variables indicadas en la tabla 5.1 se realizaron de dos modos: manteniendo la presión estática del aire en los quemadores constante y manteniendo el exceso de aire constante. La primera opción es la habitual en la industria cerámica, ya que los hornos de rodillos no disponen de un sistema de control automático que mantenga el exceso de aire constante.

Un control del aire comburente tan básico como el utilizado industrialmente implica que, en cualquier maniobra que modifique el consumo del horno, el exceso de aire se ve alterado. En otras palabras, si alguna de las modificaciones estudiadas se realizara en la industria, la variación en el consumo se debería tanto al efecto de la variable en cuestión como a la modificación en el exceso de aire. Por este motivo, con el fin de obtener resultados realistas, no sólo se contempla el caso académico en el que se mantiene el exceso de aire constante, sino también la manera en la que convencionalmente se llevaría a cabo la maniobra.

\begin{tabular}{cc} 
Parámetro & Valor \\
\hline Temperatura del aire comburente & $100{ }^{\circ} \mathrm{C}$ \\
\hline Espesor de las baldosas & $10 \mathrm{~mm}$ \\
\hline Duración del ciclo & $50 \mathrm{~min}$ \\
\hline Temperatura máxima de cocción & $1150{ }^{\circ} \mathrm{C}$ \\
\hline Contenido en $\mathrm{CaCO}_{3}$ & $13 \%$ \\
\hline
\end{tabular}

Tabla 5.1. Condiciones de referencia para las variables estudiadas. 


\subsection{Resultados}

\subsubsection{Influencia de la temperatura del aire comburente}

Se estudió la repercusión de modificar la temperatura del aire comburente en el intervalo $50-200{ }^{\circ} \mathrm{C}$ sobre el consumo energético del horno. La figura 5.3 muestra los resultados obtenidos, expresados en términos de consumo específico; esto es, la energía aportada por la combustión del gas natural (calculada a partir del PCI) por $\mathrm{m}^{2}$ de producto producido. Se realizaron cálculos para excesos de aire del $0 \%, 25 \%, 50 \%, 75 \%$ y $100 \%$ en las condiciones de referencia, en las que la temperatura del aire comburente era de $100{ }^{\circ} \mathrm{C}$ (identificada con una línea vertical en la figura 5.3). Como se ha indicado, las modificaciones con respecto a las condiciones de referencia se realizaron manteniendo constante o bien el exceso de aire o bien la presión estática del aire comburente utilizados en la situación de referencia.

Se aprecia que el exceso de aire tiene una gran influencia sobre el consumo energético. En las condiciones de referencia, operar con un $100 \%$ de exceso de aire supondría un consumo de energía un $30 \%$ superior al que se tendría si se aportara la cantidad de aire estequiométrica. Además, la dependencia es superlineal; es decir, el incremento en el consumo es menor si se pasa del $0 \%$ al $25 \%$, que si se pasa del $75 \%$ al $100 \%$.

Como era previsible, a medida que se incrementa la temperatura del aire comburente disminuye el consumo energético. Cuanto mayor es el exceso de aire, más acusada es la disminución en el consumo específico, lo cual también es lógico. Ello acarrea que la influencia del exceso del aire sea sustancialmente menor si éste estuviera precalentado a $200{ }^{\circ} \mathrm{C}$ que si se alimentara a $50^{\circ} \mathrm{C}$. Ambos efectos son deseables y, por ello, se considera que el preca-

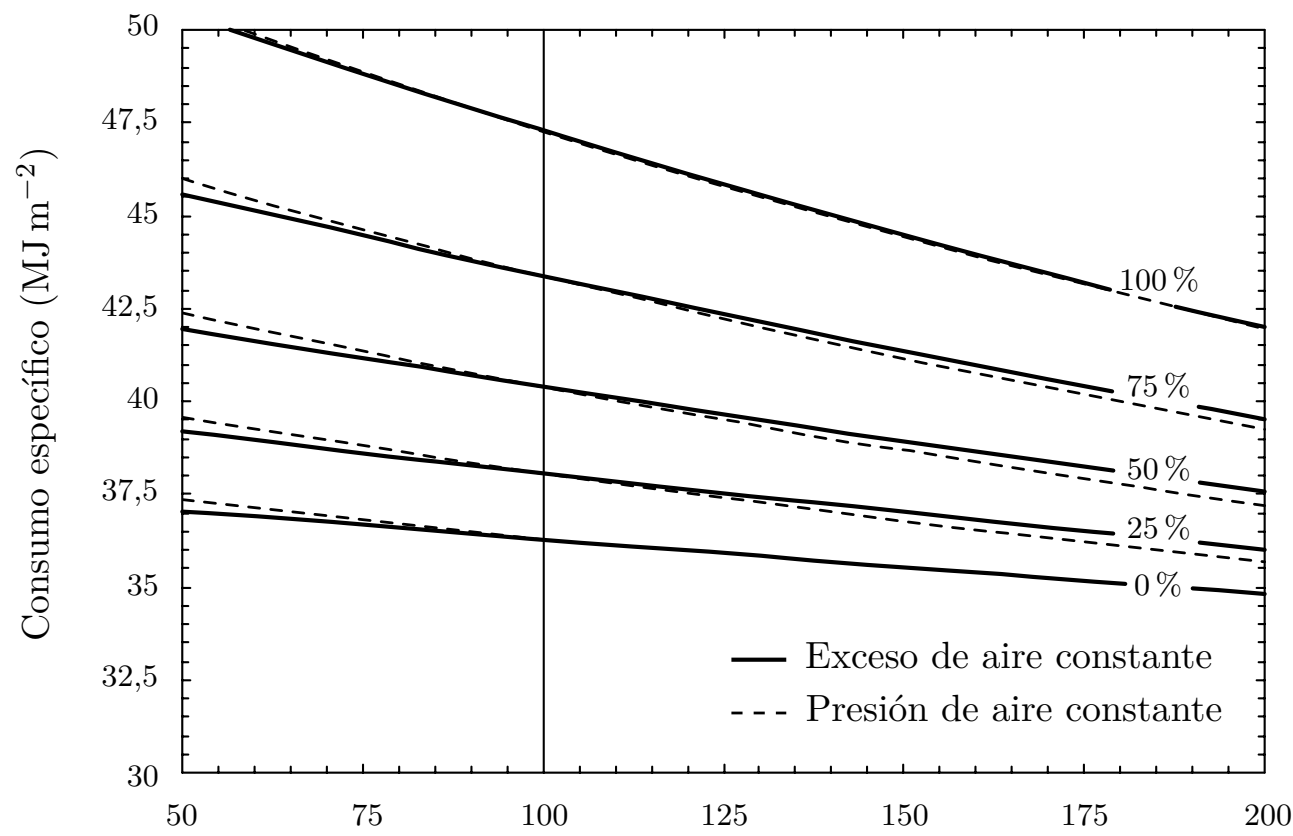

Temperatura del aire comburente $\left({ }^{\circ} \mathrm{C}\right)$

Figura 5.3. Influencia de la temperatura del aire comburente sobre el consumo energético del horno. 
lentamiento del aire comburente es una manera efectiva de reducir el consumo energético del horno, especialmente si se efectúa con calor residual del propio horno.

Para la práctica totalidad de las variables estudiadas, mantener constante la presión estática del aire comburente implica que el caudal de aire permanecerá también constante. El caso descrito en este apartado es la única excepción. El caudal de aire comburente es aproximadamente inversamente proporcional a la raíz cuadrada de la temperatura absoluta del aire, lo que significa que si la temperatura del aire comburente pasa de la temperatura $T_{a}$ a $T_{a}^{\prime}$, el caudal de aire comburente se ve modificado por el factor $\sqrt{T_{a} / T_{a}^{\prime}}$.

Si se hubiera mantenido constante el caudal de aire, en lugar de su presión estática, al disminuir el consumo energético se hubiera aumentado el exceso de aire. Sin embargo, debido a la relación que existe entre el caudal de aire y su temperatura, al aumentar la temperatura del aire sin modificar su presión estática, el exceso de aire se ve reducido. Por ejemplo, partiendo de las condiciones de referencia y un $25 \%$ de exceso de aire, si se aumentara la temperatura del aire hasta $200^{\circ} \mathrm{C}$ y se mantuviera constante su presión estática, el exceso de aire quedaría reducido al $18 \%$. En la bibliografía $[25,120]$ se identifica el posible riesgo de entrar en condiciones de defecto de aire al aumentar la temperatura del aire de combustión.

\subsubsection{Influencia del espesor de las baldosas}

La práctica totalidad de las compañías fabricantes de baldosas cerámicas incluyen productos de diferente espesor en su gama de productos, por lo que el espesor de las baldosas es una variable de proceso sujeta a frecuentes variaciones. La reducción del espesor de las baldosas conlleva una serie de ahorros en todo el proceso productivo, como por ejemplo la reducción de la cantidad de materias primas necesarias para producir una unidad de producto, e incluso en la ulterior comercialización de las baldosas, debido a su menor peso específico.

Según los cálculos realizados, el espesor tiene una influencia muy importante sobre el consumo del horno, la cual se muestra gráficamente en la figura 5.4. De las variables estudiadas, el espesor resultó ser la que más influía. A medida que se incrementa el espesor, aumenta el consumo específico de energía, especialmente si se mantiene el exceso de aire constante. La relación parece bastante lineal, pero no llega a ser de proporcionalidad directa, ni siquiera cuando se mantiene exceso de aire constante. Las curvas de exceso de aire constante son aparentemente divergentes, lo que implica que la repercusión del exceso de aire sobre el consumo se acentúa en piezas con un mayor espesor. Las curvas de presión de aire constante, por el contrario, son prácticamente paralelas dentro del intervalo de espesores estudiado.

En este caso y en los que le siguen, se considerará que mantener la presión de aire constante significa que el caudal de aire comburente permanece fijo al efectuar la modificación de la variable estudiada. Por lo tanto, si el consumo absoluto del horno (energía consumida por unidad de tiempo) se ve afectado por la modificación, el exceso de aire también varía. Al aumentar el espesor de las piezas, se incrementa el caudal másico de baldosas alimentadas al horno, por lo que el consumo absoluto del horno aumenta y, si se mantiene constante la presión estática del aire comburente, el exceso de aire disminuye. 


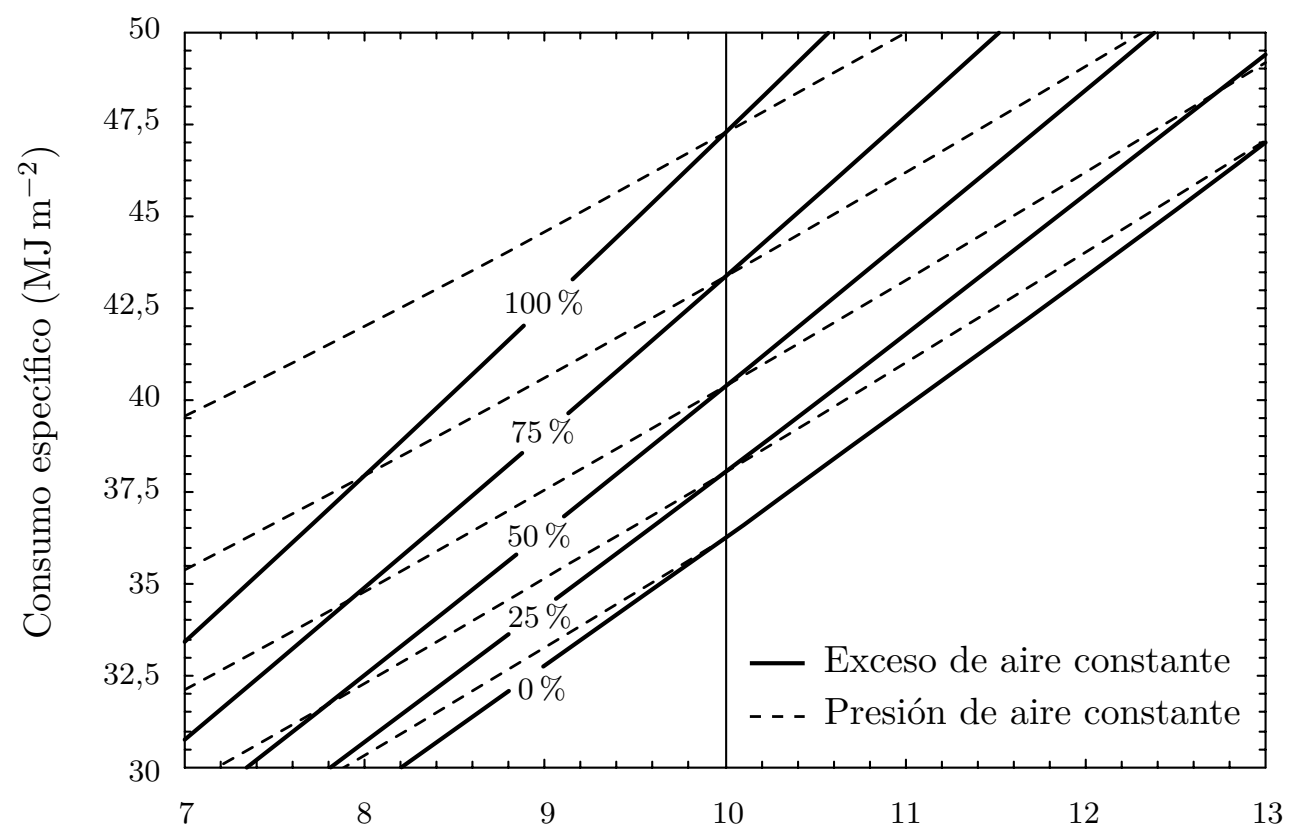

Espesor de las baldosas ( $\mathrm{mm})$

Figura 5.4. Influencia del espesor de las baldosas sobre el consumo energético del horno.

Si en las condiciones de referencia se trabajara con un $25 \%$ de exceso de aire y se redujera $2 \mathrm{~mm}$ el espesor sin modificar la presión estática del aire, el exceso de aire prácticamente se duplicaría. Si, por el contrario, se aumentara $2 \mathrm{~mm}$ el espesor de las baldosas, el exceso de aire pasaría a ser del $8 \%$. Desde el punto de vista del consumo de energía, esta última situación sería más favorable que la alternativa en la que se hubiera mantenido el exceso de aire al $25 \%$, pero podría ser la causa de defectos en el producto relacionados con la deficiente oxidación de la materia orgánica o la alteración del tono del vidriado [121].

Aparte de la notable influencia del espesor de las baldosas sobre el consumo energético del horno, esta variable afecta de manera muy marcada a la transferencia de energía en el interior de las baldosas y, por consiguiente, sobre el ciclo de temperaturas al que efectivamente quedan éstas sometidas. En la figura 5.5 (a) se muestra la evolución de la temperatura media de baldosas con diferente espesor a lo largo del horno. Se observa que las baldosas de $13 \mathrm{~mm}$ no alcanzarían la temperatura máxima de cocción durante el tiempo de permanencia a la misma, lo que, como se verá en §5.2.3, podría corregirse alargando la duración del ciclo de cocción.

En la figura 5.5 (b) se ha representado la diferencia de temperatura entre la cara superior y el centro de las baldosas a lo largo del horno para diversos espesores. Se observa que durante todo el calentamiento el centro de la pieza está más frio que la superficie. Las diferencias de temperatura en el interior de las baldosas se acrecientan a medida que se incrementa su espesor. Mientras que para las piezas de $7 \mathrm{~mm}$ estas diferencias apenas superaron los $20^{\circ} \mathrm{C}$ en los cálculos, en las piezas de $13 \mathrm{~mm}$ se alcanzaron hasta $60^{\circ} \mathrm{C}$.

En la entrada del horno, al entrar las piezas súbitamente en contacto con los gases calientes, se genera rápidamente una diferencia de temperatura entre el centro y la superficie de las 

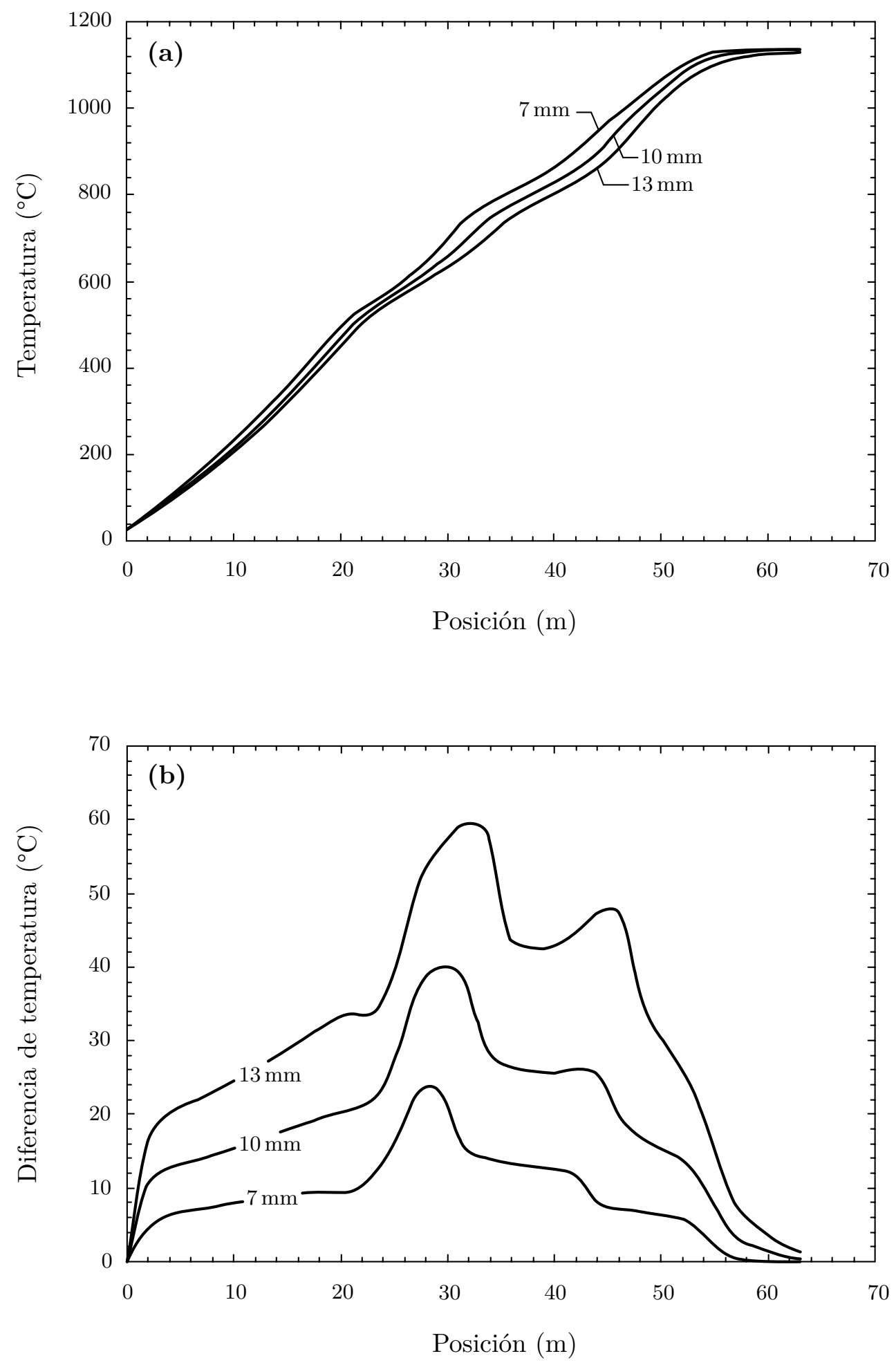

Figura 5.5. Influencia del espesor sobre: (a) la temperatura media de las baldosas y (b) la diferencia de temperatura entre la cara superior y el centro de las baldosas. 
piezas. Tras esta primera etapa, las diferencias de temperatura se incrementan paulatinamente hasta que la temperatura media de las baldosas alcanza unos $500{ }^{\circ} \mathrm{C}$, momento en el que aumentan drásticamente las diferencias de temperatura entre el centro y la superficie. Esto es consecuencia del inicio de la deshidroxilación del material arcilloso que, al absorber calor, tiene el efecto de prevenir el calentamiento del interior de la pieza. A este primer pico le sigue un segundo, relacionado con la descomposición de los carbonatos. Finalmente, las temperaturas en el interior de la pieza se van igualando, coincidiendo con el periodo de permanencia a la temperatura máxima. En un estudio experimental [29] se obtuvieron resultados similares.

\subsubsection{Influencia de la duración del ciclo de cocción}

Está generalmente admitido que al alargar la duración del ciclo se incrementa el consumo específico de energía. Esta conclusión se basa en que en el balance global de energía surgen una serie de términos (principalmente las pérdidas de energía a través de las paredes) que apenas deberían depender del caudal másico de baldosas alimentadas al horno $[13,120]$.

La figura 5.6 recoge los consumos energéticos del horno obtenidos para diferentes duraciones del ciclo de cocción. Las curvas de exceso de aire constante exhiben un mínimo. Para ciclos de cocción largos el consumo se incrementa debido a que adoptan más peso en el balance los términos que no dependen del caudal másico de baldosas, lo que concuerda con la predicción arriba indicada. Sin embargo, para ciclos de cocción muy rápidos, se produce un aumento en el consumo debido a que los gases abandonan el horno a una mayor

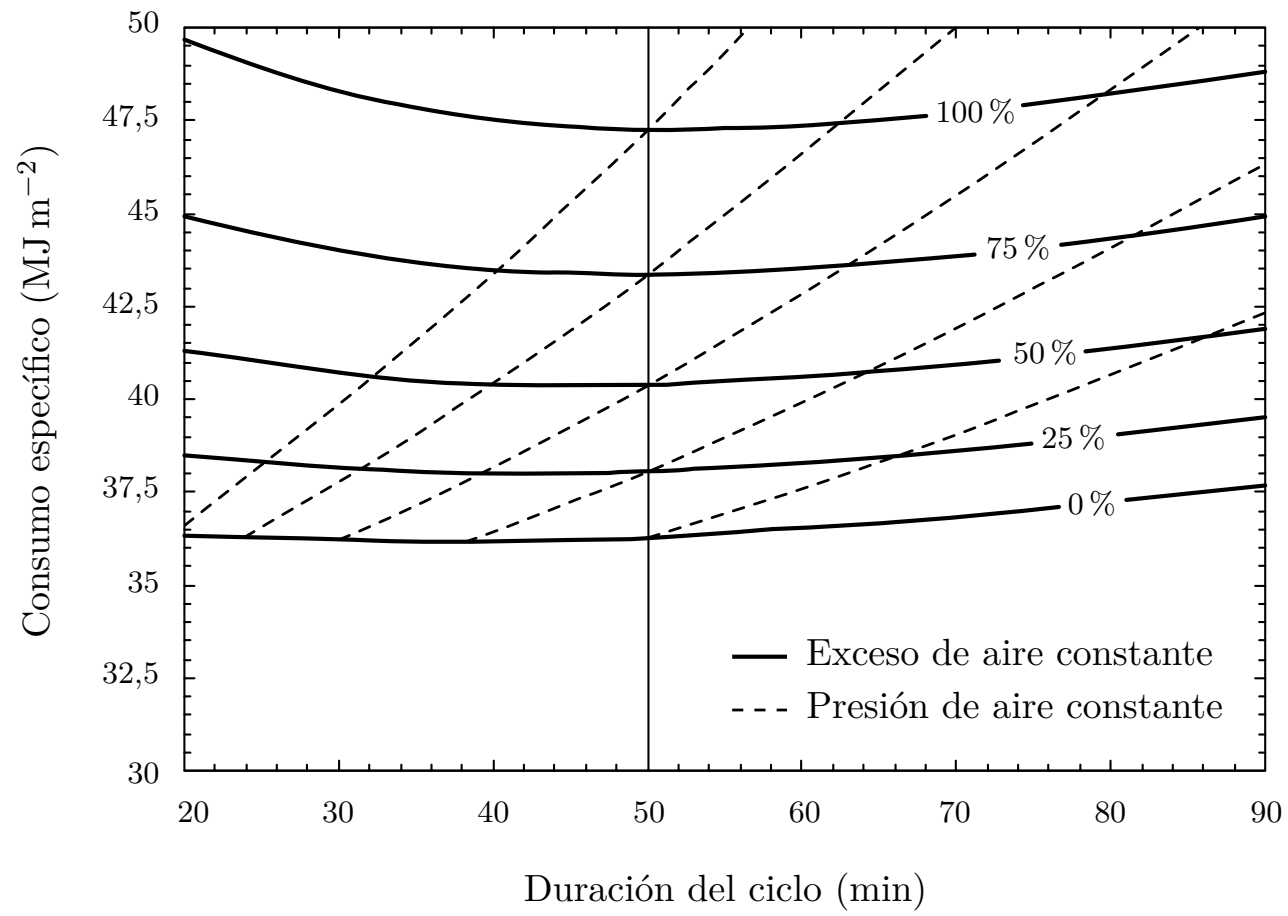

Figura 5.6. Influencia de la duración del ciclo de cocción sobre el consumo energético del horno. 


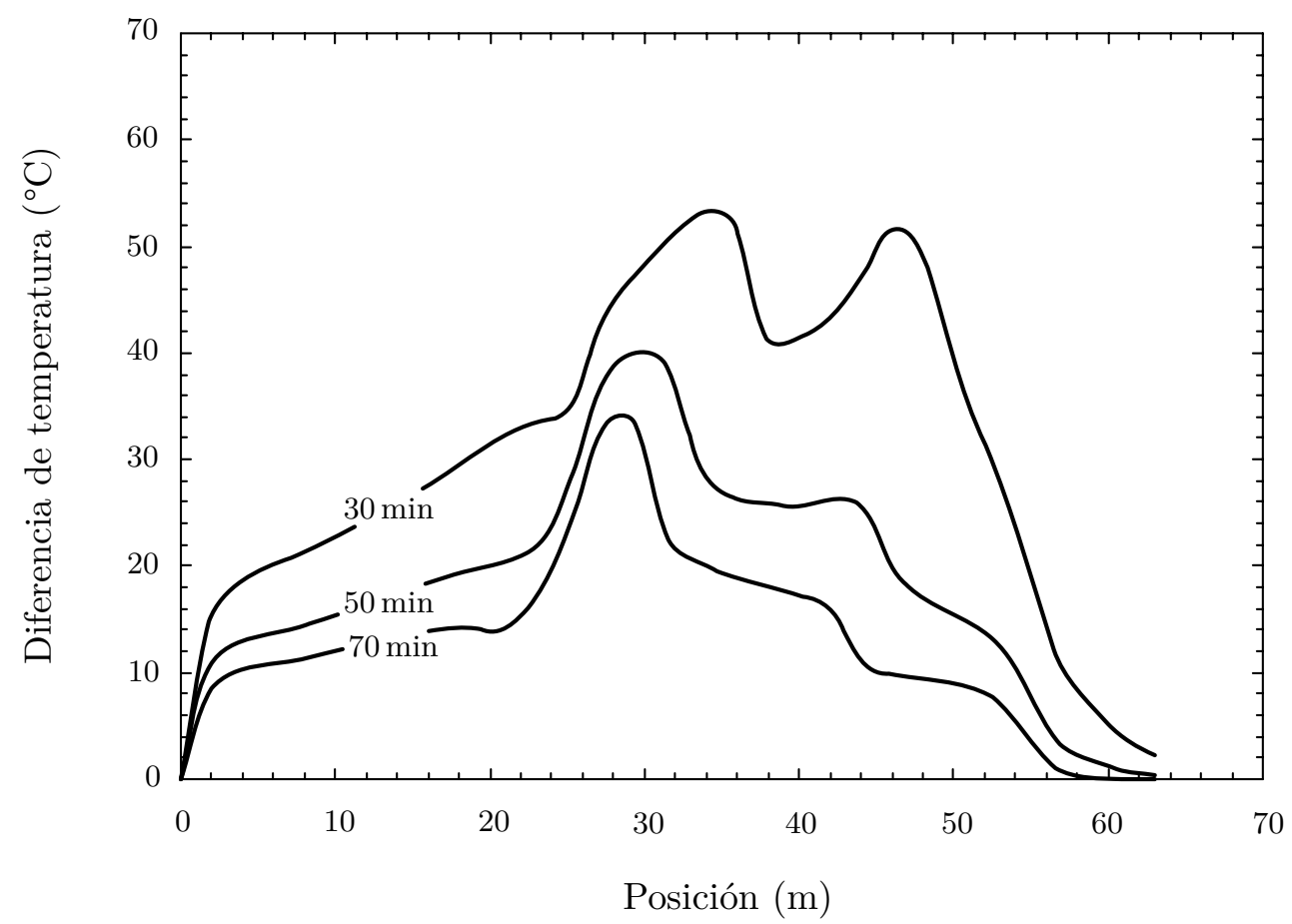

Figura 5.7. Influencia de la duración del ciclo sobre la diferencia de temperatura entre la cara superior y el centro de las baldosas.

temperatura. Cuanto mayor es el exceso de aire, más desplazado está el mínimo hacia la derecha.

Si se mantiene la presión del aire constante, la situación es manifiestamente diferente. En este caso sí que se observa un aumento continuo del consumo específico con la duración del ciclo. Al acortar la duración del ciclo (incrementar la producción), el consumo de gas natural por parte del horno (en términos absolutos) aumenta, debido al mayor caudal másico de baldosas a tratar térmicamente. Si no se modifica el caudal de aire comburente, se producirá una disminución del exceso de aire, por lo que el consumo específico disminuye. Por lo tanto, este efecto no es realmente debido a la modificación de la duración del ciclo, sino a la variación del exceso de aire. Es posible que algunos resultados observados en la industria se hayan atribuido incorrectamente a la duración del ciclo sin haber tenido la precaución de mantener el exceso de aire constante.

La duración del ciclo de cocción también tiene influencia sobre la distribución interna de temperatura en las baldosas. La figura 5.7 muestra las diferencias de temperaturas entre la superficie superior y el centro de las baldosas para duraciones del ciclo de cocción de $30 \mathrm{~min}, 50 \mathrm{~min}$ y $70 \mathrm{~min}$. A medida que se acorta la duración del ciclo, se incrementan las diferencias de temperatura.

El espesor de las baldosas y la duración del ciclo están bastante ligadas en la operación de cocción. Por una parte, las baldosas de mayor espesor requieren un ciclo de cocción más largo, por lo que cabría esperar que industrialmente se modifiquen ambas simultáneamente. Por otra parte, los motivos por los que se les atribuye una influencia en el consumo energético del horno son competitivos. 
En el caso límite en el que el consumo del horno no dependiera del caudal másico de baldosas a procesar, el consumo específico sería directamente proporcional a la duración del ciclo y el espesor no tendría influencia alguna. En el extremo opuesto, el consumo específico de energía sería directamente proporcional al espesor e independiente de la duración del ciclo. Los cálculos realizados sugieren que la realidad está más próxima a esta segunda situación. Ahora bien, para composiciones que requieran un menor calor de reacción (gres o porcelánico), baldosas de espesor muy reducido u hornos con mayores pérdidas por paredes, la influencia de la duración del ciclo podría cobrar una mayor importancia.

\subsubsection{Influencia de la temperatura máxima de cocción}

El efecto de modificar la temperatura máxima de cocción (manteniendo el resto de características del ciclo) sobre el consumo específico se muestra en la figura 5.8. Dentro de los márgenes de variación de la temperatura máxima estudiados, las variaciones asociadas en el consumo resultaron ser moderadas. Como cabría esperar, el consumo disminuye con la temperatura máxima de cocción, pero una disminución de $100^{\circ} \mathrm{C}$ apenas modificaría el consumo en un $6-7 \%$.

Para maximizar los beneficios de disminuir la temperatura máxima de cocción debe mantenerse el exceso de aire constante. En caso contrario, si no se modifica la presión estática del aire, la disminución en el consumo se ve atenuada por un incremento en el exceso de aire. Este efecto es tanto más importante cuanto mayor es el exceso de aire en la situación de partida.

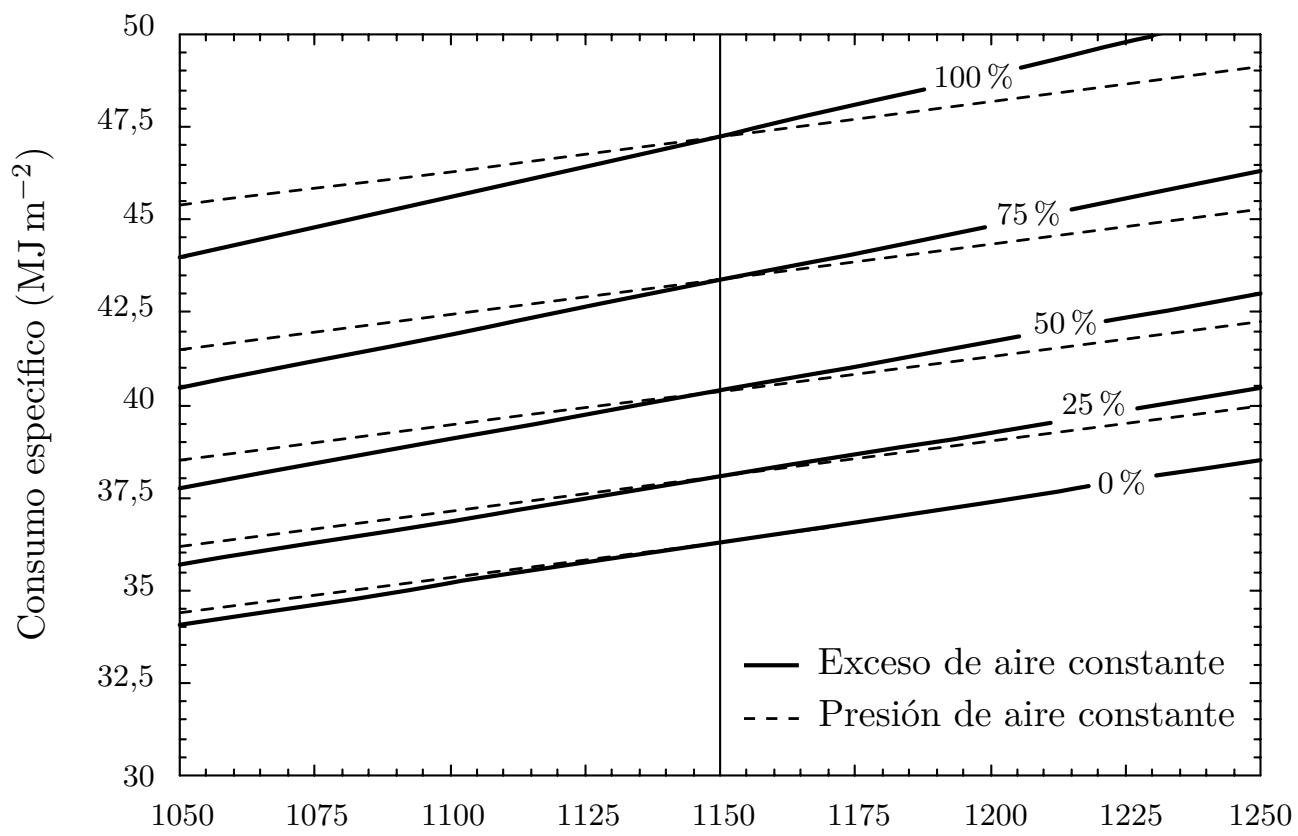

Temperatura máxima de cocción $\left({ }^{\circ} \mathrm{C}\right)$

Figura 5.8. Influencia de la temperatura máxima de cocción sobre el consumo energético del horno. 


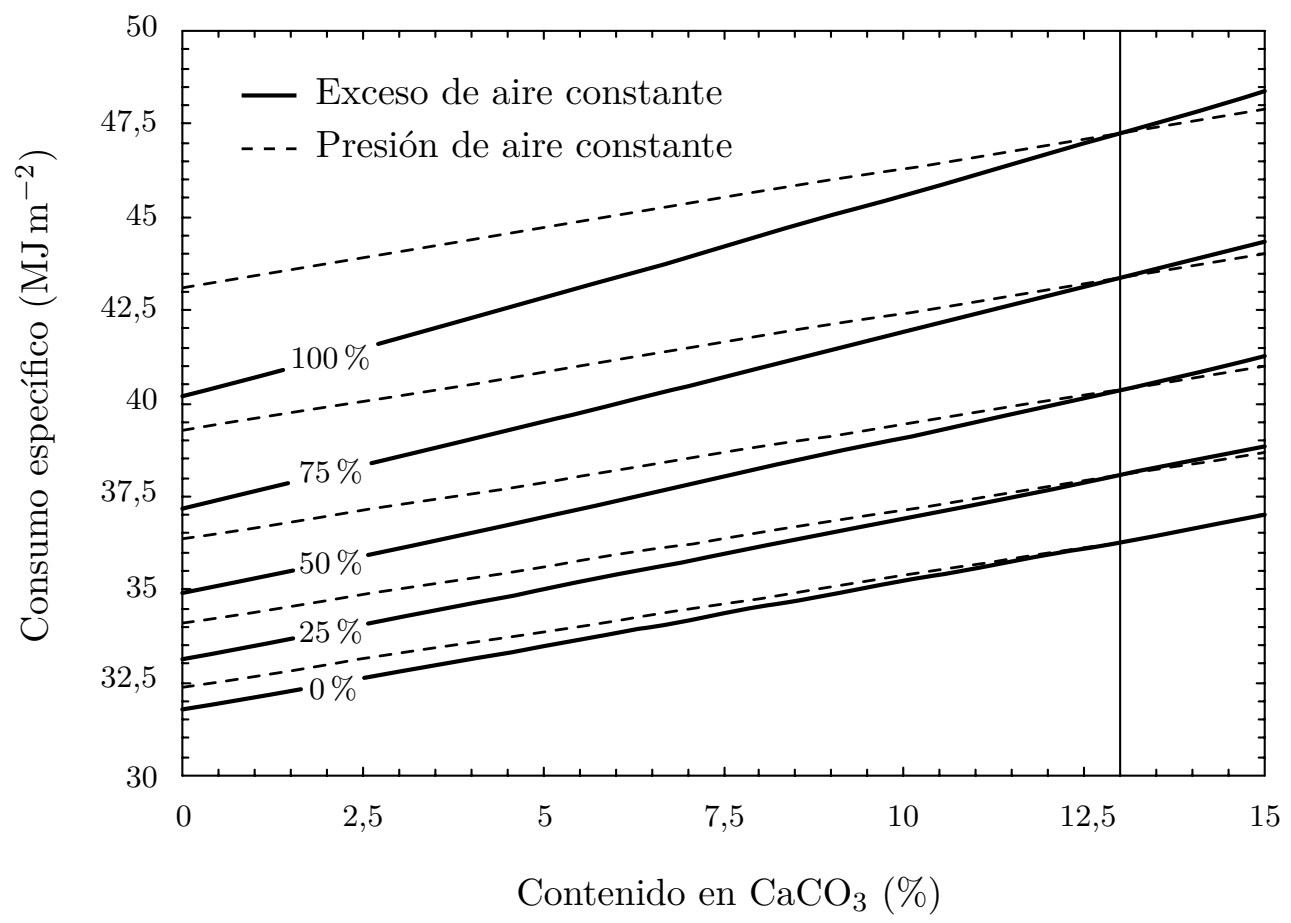

Figura 5.9. Influencia del contenido en carbonatos sobre el consumo energético del horno.

\subsubsection{Influencia del contenido en carbonatos en la composición cerámica}

Se estudió el efecto de una reducción parcial del contenido en carbonatos en la composición por otra hipotética materia prima que aporte un comportamiento similar en la cocción, pero que no experimente transformaciones significativamente endotérmicas. En este sentido, la wollastonita se ha propuesto como una alternativa a los carbonatos $[122,123]$.

En la figura 5.9 se muestra la variación calculada del consumo energético del horno al modificar el contenido en carbonatos en la composición. Como puede verse, a medida que se reduce el contenido en carbonatos también lo hace el consumo energético, especialmente si se corrige el aumento del exceso de aire derivado del menor consumo del horno. Según los cálculos realizados, la eliminación total de los carbonatos podría suponer una reducción del consumo de alrededor de un $13 \%$ en las condiciones estudiadas. Además, la eliminación de los carbonatos podría hacer innecesaria la etapa de descomposición identificada en la figura 5.2, lo que a su vez podría repercutir en un efecto positivo sobre el consumo. 



\section{Conclusiones y recomendaciones}

\subsection{Conclusiones}

La ejecución del presente trabajo ha permitido obtener las siguientes conclusiones:

- Se ha desarrollado un modelo matemático que describe la transferencia de energía en la cocción de baldosas cerámicas en hornos de rodillos. El modelo desarrollado permite calcular (§3.4 y §3.5):

- Las temperatura de todas las zonas en las que se divida el horno, incluida la distribución interna de temperaturas en las baldosas procesadas y en las paredes del horno.

- Los flujos de energía para cada zona, distinguiendo el mecanismo de transporte de calor: convección, conducción, radiación o flujo de entalpía. A partir de dichos flujos, es inmediato calcular las entradas y salidas de energía en un entorno cualquiera que incluya todas o parte de las zonas.

- La regulación de quemadores con la que se satisface una curva de cocción predefinida. En otras palabras, es posible predecir tanto el caudal total de gas natural aportado al horno (consumo energético), como el caudal individual en cada anillo de quemadores.

- La composición química de los gases que circulan por el horno en cualquier posición a lo largo del mismo, teniendo en cuenta incluso las desgasificaciones de las baldosas.

La utilidad de dicha información para el diseño del horno (y sus equipos auxiliares) es evidente. Además, el modelo permite estimar el valor de variables cuya determinación experimental es muy difícil (por no decir imposible), como puede ser la temperatura en el interior de las baldosas. La disponibilidad de dicha información hace posible realizar cálculos subsiguientes, como por ejemplo el cálculo de tensiones y deformaciones en las baldosas [27, 124, 125].

- El desarrollo del modelo ha traído consigo una serie de innovaciones que trascienden el ámbito para el que fueron desarrolladas y, por tanto, podrían ser de utilidad en otros estudios:

- Se ofrece un catálogo de fórmulas explícitas para el cálculo de las DEA en el horno de rodillos (§3.2.2, §3.2.3 y §3.2.4), expresadas en términos de integrales simples o dobles. Combinadas con un algoritmo de integración eficiente, estas fórmulas permiten calcular las DEA mucho más rápido que el método de Monte Carlo y podrían ser de aplicación para el análisis térmico de equipos industriales que guarden similitudes geométricas con el horno de rodillos. 
- Se ha ideado un algoritmo de integración adaptativo que permite evaluar integrales impropias de segunda especie (§3.2.5), con el cual se pueden calcular eficientemente las DEA indicadas en el punto anterior. El algoritmo es de aplicación general: no se limita al cálculo de las DEA.

- Se ha corroborado la validez de las hipótesis adoptadas para el desarrollo del modelo en tres hornos industriales, con características dispares en cuanto a longitud, productos procesados y condiciones de operación (§4.1). La recopilación de datos en dichas instalaciones permitió realizar el cálculo de las temperaturas de las zonas en las que se dividieron los hornos, las cuales pudieron compararse con valores experimentales. En todos los casos estudiados, se obtuvo un acuerdo aceptable entre las temperaturas experimentales y las calculadas (§4.2).

- Se ha utilizado el modelo para estimar la influencia de una selección de variables de proceso sobre el consumo energético de un horno hipotético, obteniéndose las siguientes conclusiones:

- El exceso de aire tiene una notable influencia sobre el consumo energético del horno. Además, dado que el control del caudal de aire comburente se realiza de una manera básicamente manual, al realizar una maniobra que afecte al consumo, el exceso de aire puede verse modificado (§5.1). En tal caso, la variación en el consumo observada se debería a la acción combinada de ambos efectos.

- Aumentar la temperatura del aire comburente disminuye el consumo energético del horno (§5.2.1). Al implantar dicha medida de ahorro energético debe tenerse presente que, aún habiendo una disminución en el caudal de combustible, si no se modifica la presión estática del aire comburente, el exceso de aire se reduce por la relación que existe entre el caudal de aire en el quemador y su temperatura.

- El espesor de las baldosas influye en gran medida sobre el consumo del horno (§5.2.2). Cuanto mayor es el espesor, más energía se requiere para efectuar la cocción de una unidad funcional de producto. Además, si se emplea la misma presión estática del aire comburente para la cocción de productos con diferente espesor, las condiciones de exceso de aire - y, por tanto, la presión parcial de $\mathrm{O}_{2}$ en los gases del horno - serán distintas para cada producto, dependiendo de su espesor.

- Pese a que está generalmente aceptado que el consumo específico de energía aumenta monótonamente con la duración del ciclo, este comportamiento se verifica únicamente si el caudal de aire comburente permanece constante (§5.2.3). $\mathrm{Si}$, por el contrario, se mantiene el exceso de aire constante, las curvas de consumo específico frente a duración del ciclo describen un mínimo. En los ciclos muy cortos se produce un peor aprovechamiento del contenido energético de los gases y, por ello, no resultan óptimos desde el punto de vista energético.

- Una mayor temperatura máxima de cocción involucra consumos energéticos más elevados (\$5.2.4), pero considerando variaciones realistas para esta variable de proceso, su influencia debería ser únicamente relevante cuando se comparan productos de distinto tipo (azulejo frente a porcelánico), que es cuando se tienen diferencias significativas en la temperatura máxima de cocción. 
- La presencia de carbonatos en la composición cerámica incrementa de manera significativa el consumo de energía en la cocción (\$5.2.5). Este hecho podría justificar el mayor consumo por unidad de masa de los azulejos, cuya fórmula involucra una mayor proporción de carbonatos.

- Considerando las posibilidades de variación de las variables estudiadas a nivel industrial, el espesor de las piezas y el exceso de aire son las que mayor influencia tienen sobre el consumo de energía en la cocción.

\subsection{Recomendaciones}

El trabajo desarrollado resuelve muchas cuestiones, pero también permite establecer las siguientes recomendaciones que podrían ser objeto de un estudio posterior:

- La operación de cocción de baldosas cerámicas en hornos monoestrato de rodillos actuales está sujeta a un control del exceso de aire demasiado rudimentario. Se recomienda incorporar sistemas de medida en continuo de los caudales de combustible y comburente en los quemadores y/o del contenido en $\mathrm{O}_{2}$ en los humos. Idealmente, la información registrada con dichos sensores debería alimentarse a un lazo de control automático del exceso de aire.

- En este trabajo se ha simulado un conjunto limitado de casos relacionados con la modificación de algunas de las variables de proceso más importantes. Las posibilidades del modelo son mucho más amplias. En particular, podría efectuarse una continuación del presente trabajo en la que se estudiaran tecnologías innovadoras, como por ejemplo la sustitución del gas natural por otros combustibles o el uso de oxicombustión, por mencionar algunas.

- En el calentamiento tienen lugar los principales cambios microestructurales en las baldosas y es donde se produce el consumo de gas natural. Sin embargo, el enfriamiento también tiene mucha importancia en la operación de cocción. Por una parte, en el enfriamiento ocurren fenómenos relevantes como el acoplamiento entre el soporte y el vidriado, o la transformación polimórfica del cuarzo. Por otra parte, el aprovechamiento del calor sensible de las baldosas es esencial para incrementar el rendimiento energético del horno. Se plantea, como una línea futura de investigación, extender el modelo para poder simular también el enfriamiento. 



\section{A. Apéndice}

\section{A.1. Funciones especiales}

\section{A.1.1. Integral exponencial}

La integral exponencial general se define como:

$$
\mathrm{E}_{n}(x)=\int_{1}^{\infty} \frac{\mathrm{e}^{-x t}}{t^{n}} \mathrm{~d} t=x^{n-1} \int_{x}^{\infty} \frac{\mathrm{e}^{-t}}{t^{n}} \mathrm{~d} t
$$

pero la variante que recibe el nombre de «integral exponencial» y que tiene una notación específica es:

$$
\operatorname{Ei}(x)=-\int_{-x}^{\infty} \frac{\mathrm{e}^{-t}}{t} \mathrm{~d} t=-\mathrm{E}_{1}(-x)
$$

Las integrales exponenciales generales obedecen la propiedad de recurrencia:

$$
\mathrm{E}_{n+1}(x)=\frac{1}{n}\left(\mathrm{e}^{-x}-x \mathrm{E}_{n}(x)\right)
$$

\section{A.1.2. Función gamma incompleta}

La función gamma incompleta se define como:

$$
\Gamma_{n}(x)=\int_{x}^{\infty} t^{n-1} \mathrm{e}^{-t} \mathrm{~d} t
$$

que se puede generalizar:

$$
\Gamma_{n}\left(x_{0}, x_{1}\right)=\int_{x_{0}}^{x_{1}} t^{n-1} \mathrm{e}^{-t} \mathrm{~d} t=\Gamma_{n}\left(x_{0}\right)-\Gamma_{n}\left(x_{1}\right)
$$

La función gamma incompleta está relacionada con la integral exponencial general:

$$
\mathrm{E}_{n}(x)=x^{n-1} \Gamma_{1-n}(x)
$$

y se puede calcular recurrentemente mediante:

$$
\Gamma_{n+1}(x)=n \Gamma_{n}(x)+x^{n} \mathrm{e}^{-x}
$$




\section{A.1.3. Integral secante generalizada}

La integral secante generalizada se define como:

$$
\mathrm{S}_{n}(x, \Psi)=x^{n} \int_{0}^{\Psi} \mathrm{e}^{-x \sec \psi} \sec ^{n} \psi \mathrm{d} \psi
$$

El caso particular $\mathrm{S}_{0}(b, \Psi)$ se llama integral de Sievert [126].

Las integrales secantes generalizadas pueden aproximarse por las series [127]:

$$
\mathrm{S}_{n}(x, \Psi)= \begin{cases}\mathrm{e}^{-x} \sum_{i=0}^{\infty} p_{n i} x^{n-i-\frac{1}{2}} \Gamma_{i+\frac{1}{2}}(0, x(\sec \Psi-1)) & \text { si } \Psi \leq \frac{\pi}{4} \\ \mathrm{~S}_{n}\left(x, \frac{\pi}{4}\right)+\sum_{i=0}^{\infty} k_{i} x^{2 i+1} \Gamma_{n-2 i-1}(x \sqrt{2}, x \sec \Psi) & \text { si } \frac{\pi}{4}<\Psi \leq \frac{\pi}{2}\end{cases}
$$

en la que:

$$
\begin{aligned}
& p_{n i}=\frac{1}{\sqrt{2}} \sum_{j=0}^{i}\left(\frac{-1}{2}\right)^{j}\left(\begin{array}{c}
n-1 \\
i-j
\end{array}\right) \frac{(2 j-1) ! !}{(2 j) ! !} \\
& k_{i}=\frac{(2 i-1) ! !}{(2 i) ! !}
\end{aligned}
$$

Según Michieli [127], tomando 3 términos de estas expansiones se consigue una precisión mejor del $1 \%$.

\section{A.1.4. Otras funciones}

En este apartado se incluyen ciertas funciones que, si bien no deberían calificarse de «especiales», sí que son lo bastante poco frecuentes como para que sea apropiado definirlas. Estas funciones son:

- Función suelo:

$$
\lfloor x\rfloor=\operatorname{máx}\{n \in \mathbb{Z} \mid n \leq x\}
$$

- Función de Heaviside:

$$
\mathrm{H}(x)= \begin{cases}0 & \text { si } x \leq 0 \\ 1 & \text { si } x>0\end{cases}
$$

- Corchetes de Macaulay:

$$
\langle x\rangle= \begin{cases}0 & \text { si } x \leq 0 \\ x & \text { si } x>0\end{cases}
$$



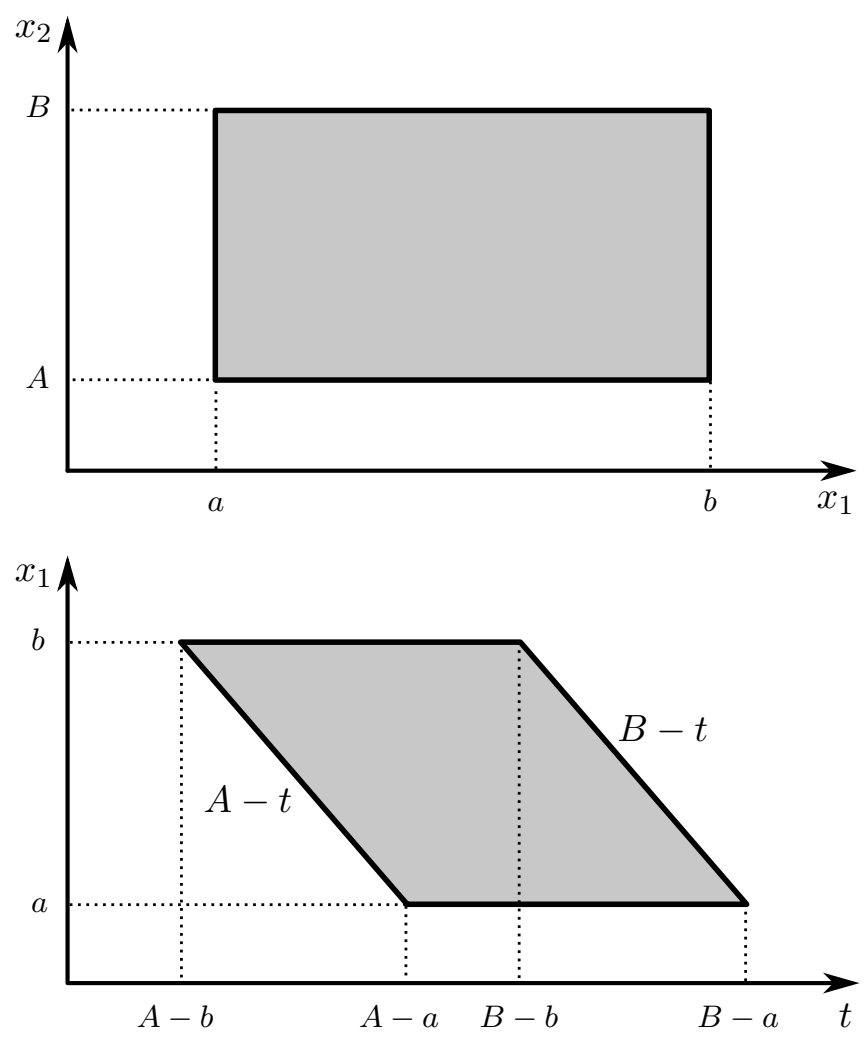

Figura A.1. Dominio de integración tras la aplicación del cambio de Mohr.

\section{A.2. Cambios de variables útiles en el desarrollo de las DEA}

\section{A.2.1. Cambio de Mohr}

Las integrales dobles del tipo:

$$
I=\int_{A}^{B} \int_{a}^{b} f\left(x_{2}-x_{1}\right) \mathrm{d} x_{1} \mathrm{~d} x_{2}
$$

pueden reducirse a una suma de tres integrales simples gracias al siguiente cambio de variables que Erkku [85] atribuye a W. D. Mohr:

$$
t=x_{2}-x_{1}
$$

Tras la aplicación del cambio de variables, el dominio de integración debe modificarse de la manera indicada en la figura A.1. La integral doble (A.15) puede expresarse como la suma de tres integrales:

$$
I=\int_{A-b}^{A-a} \int_{A-t}^{b} f(t) \mathrm{d} x_{1} \mathrm{~d} t+\int_{A-a}^{B-b} \int_{a}^{b} f(t) \mathrm{d} x_{1} \mathrm{~d} t+\int_{B-b}^{B-a} \int_{a}^{B-t} f(t) \mathrm{d} x_{1} \mathrm{~d} t
$$


Integrando respecto de $x_{1}$ :

$$
I=\int_{A-b}^{A-a}(b-A+t) f(t) \mathrm{d} t+\int_{A-a}^{B-b}(b-a) f(t) \mathrm{d} t+\int_{B-b}^{B-a}(B-a-t) f(t) \mathrm{d} t
$$

En caso de que $a=A$ y $b=B$ (situación bastante frecuente en las DEA del horno de rodillos), pueden unificarse las tres integrales:

$$
I=2 \int_{0}^{b-a}(b-a-t) f(t) \mathrm{d} t
$$

siempre que $f(t)$ sea simétrica respecto de $t=a$.

\section{A.2.2. Cambio de Ström}

Este cambio de variables, que también permite expresar una integral doble como una suma de integrales simples, usualmente se atribuye a Siddall [128], pero es realmente debido a Ström [129].

Considérese la integral de la forma:

$$
I=\int_{A}^{B} \int_{a}^{b} f\left(x_{1}, x_{2}\right) g\left(\sqrt{x_{1}^{2}+x_{2}^{2}}\right) \mathrm{d} x_{1} \mathrm{~d} x_{2}
$$

Haciendo el cambio a polares:

$$
\begin{aligned}
& x_{1}=\rho \cos \alpha \\
& x_{2}=\rho \operatorname{sen} \alpha
\end{aligned}
$$

y teniendo en cuenta la modificación en el dominio de integración (figura A.2):

$$
I=\int_{\sqrt{a^{2}+A^{2}}}^{\sqrt{a^{2}+B^{2}}} \varphi_{1}(\rho) g(\rho) \rho \mathrm{d} \rho+\int_{\sqrt{a^{2}+B^{2}}}^{\sqrt{A^{2}+b^{2}}} \varphi_{2}(\rho) g(\rho) \rho \mathrm{d} \rho+\int_{\sqrt{A^{2}+b^{2}}}^{\sqrt{A^{2}+B^{2}}} \varphi_{3}(\rho) g(\rho) \rho \mathrm{d} \rho
$$

donde:

$$
\begin{aligned}
\varphi_{1}(\rho) & =\int_{\operatorname{arcsen} \frac{A}{\rho}}^{\arccos \frac{a}{\rho}} f(\rho, \alpha) \mathrm{d} \alpha \\
\varphi_{2}(\rho) & =\int_{\operatorname{arcsen} \frac{A}{\rho}}^{\operatorname{arcsen} \frac{B}{\rho}} f(\rho, \alpha) \mathrm{d} \alpha \\
\varphi_{3}(\rho) & =\int_{\arccos \frac{b}{\rho}}^{\operatorname{arcsen} \frac{B}{\rho}} f(\rho, \alpha) \mathrm{d} \alpha
\end{aligned}
$$



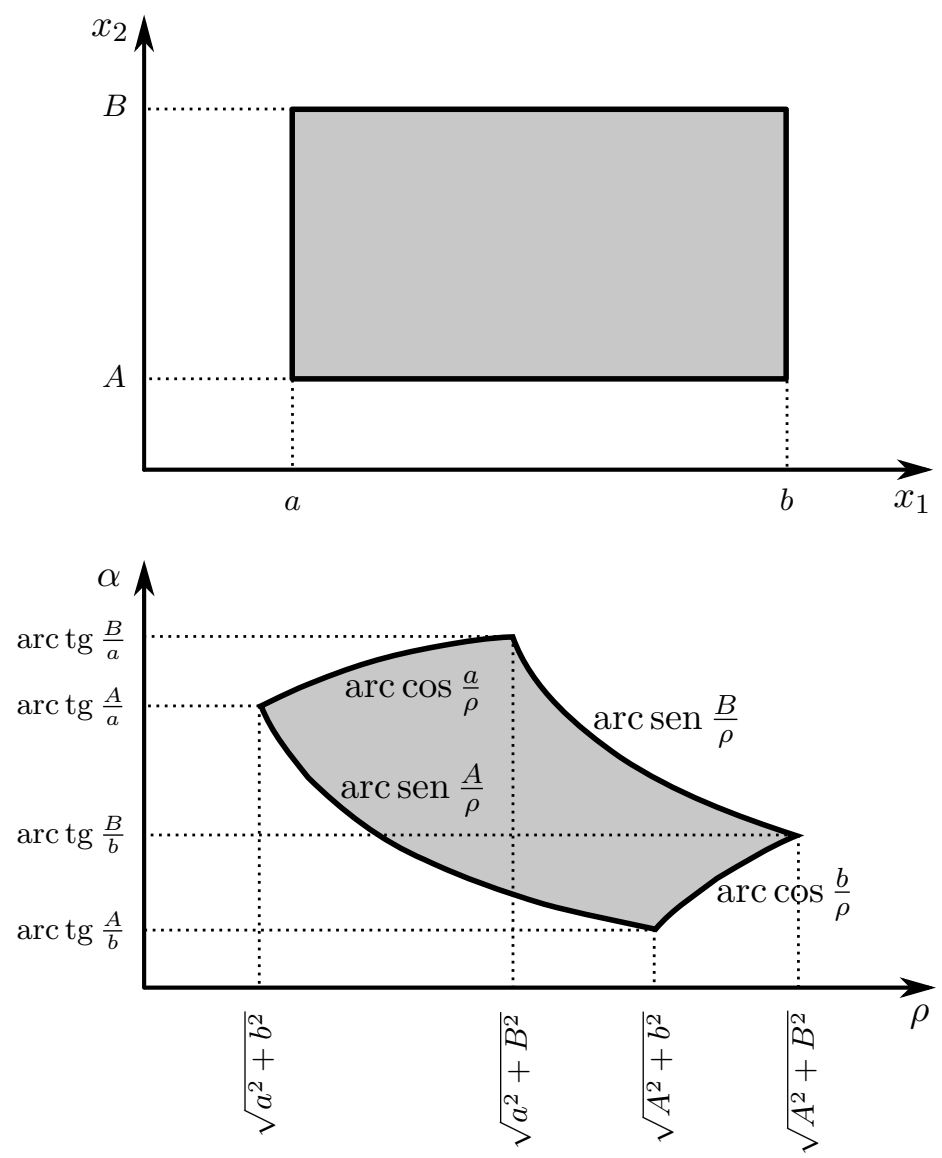

Figura A.2. Dominio de integración tras la aplicación del cambio de Ström.

\section{A.3. Integrales útiles en el desarrollo de las DEA}

Las siguientes integrales surgen profúsamente en el desarrollo de las DEA:

$$
\begin{aligned}
& \int_{0}^{L} t \frac{\mathrm{e}^{-K \sqrt{t^{2}+\rho^{2}}}}{\left(\sqrt{t^{2}+\rho^{2}}\right)^{n}} \mathrm{~d} t=K^{n-2} \Gamma_{2-n}\left(K \rho, K \sqrt{L^{2}+\rho^{2}}\right) \\
& \int_{0}^{L} \frac{\mathrm{e}^{-K \sqrt{t^{2}+\rho^{2}}}}{\left(\sqrt{t^{2}+\rho^{2}}\right)^{n}} \mathrm{~d} t=\frac{K^{n-2}}{\rho} \mathrm{S}_{2-n}\left(K \rho, \operatorname{arctg} \frac{L}{\rho}\right)
\end{aligned}
$$

Si $n>2$, la ecuación (A.27) involucra integrales secantes que no se pueden calcular mediante las series definidas en §A.1.3 [130]. No obstante, estas integrales pueden expresarse en términos de otras integrales para las que $n \leq 2$, mediante la fórmula de recurrencia que se presenta seguidamente.

Sea:

$$
I_{m, n}=\int t^{m} \frac{\mathrm{e}^{-K \sqrt{t^{2}+\rho^{2}}}}{\left(\sqrt{t^{2}+\rho^{2}}\right)^{n}} \mathrm{~d} t
$$


y:

$$
C_{m, n}=t^{m} \frac{\mathrm{e}^{-K \sqrt{t^{2}+\rho^{2}}}}{\left(\sqrt{t^{2}+\rho^{2}}\right)^{n}}
$$

Combinando la integral por partes:

$$
(m+1) I_{m, n}=C_{m+1, n}+K I_{m+2, n+1}+n I_{m+2, n+2}
$$

con el desarrollo aritmético:

$$
I_{m+2, n}=I_{m, n-2}-\rho^{2} I_{m, n}
$$

se llega a la fórmula de recurrencia:

$$
n \rho^{2} I_{m, n+2}+K \rho^{2} I_{m, n+1}+(m+1-n) I_{m, n}-K I_{m, n-1}=C_{m+1, n}
$$

\section{A.4. Desarrollo de las DEA}

\section{A.4.1. Intercambio entre volúmenes de gas}

El DEA entre los volúmenes de gas de la figura 3.4 viene dada por:

$$
\overline{g_{i} g_{j}}=\int_{0}^{L_{3}} \int_{0}^{L_{3}} \int_{0}^{L_{2}} \int_{0}^{L_{2}} \int_{0}^{d+L_{1}^{(j)}} \int_{0}^{L_{1}^{(i)}} \frac{K^{2}}{\pi r^{2}} \mathrm{e}^{-K r} \mathrm{~d} x_{i} \mathrm{~d} x_{j} \mathrm{~d} y_{i} \mathrm{~d} y_{j} \mathrm{~d} z_{i} \mathrm{~d} z_{j}
$$

siendo:

$$
r=\sqrt{\left(x_{j}-x_{i}\right)^{2}+\left(y_{j}-y_{i}\right)^{2}+\left(z_{j}-z_{i}\right)^{2}}
$$

Aplicando tres veces el cambio de Mohr $\left(t_{1}=x_{j}-x_{i}, t_{2}=y_{j}-y_{i}\right.$ y $\left.t_{3}=z_{j}-z_{i}\right)$ se llega a:

$$
\begin{aligned}
\overline{g_{i} g_{j}} & =\int_{d-L_{1}^{(i)}}^{d}\left(L_{1}^{(i)}-d+t_{1}\right) f\left(t_{1}\right) \mathrm{d} t_{1}+\int_{d}^{d+L_{1}^{(j)}-L_{1}^{(i)}} L_{1}^{(i)} f\left(t_{1}\right) \mathrm{d} t_{1}+ \\
& +\int_{d+L_{1}^{(j)}-L_{1}^{(i)}}^{d+L_{1}^{(j)}}\left(L_{1}^{(j)}+d-t_{1}\right) f\left(t_{1}\right) \mathrm{d} t_{1}
\end{aligned}
$$

siendo:

$$
f\left(t_{1}\right)=\frac{4 K^{2}}{\pi} \int_{0}^{L_{3}} \int_{0}^{L_{2}}\left(L_{2}-t_{2}\right)\left(L_{3}-t_{3}\right) \frac{\mathrm{e}^{-K \sqrt{t_{1}^{2}+t_{2}^{2}+t_{3}^{2}}}}{t_{1}^{2}+t_{2}^{2}+t_{3}^{2}} \mathrm{~d} t_{2} \mathrm{~d} t_{3}
$$


Integrando respecto de $t_{3}$ :

$$
f\left(t_{1}\right)=\frac{4 K^{2}}{\pi} \int_{0}^{L_{2}}\left(L_{2}-t_{2}\right)\left(\frac{L_{3}}{\rho} \mathrm{S}_{0}\left(K \rho, \operatorname{arctg} \frac{L_{3}}{\rho}\right)-\Gamma_{0}\left(K \rho, K \sqrt{L_{3}^{2}+\rho^{2}}\right)\right) \mathrm{d} t_{2}
$$

donde:

$$
\rho=\rho\left(t_{1}, t_{2}\right)=\sqrt{t_{1}^{2}+t_{2}^{2}}
$$

Denotando:

$$
I_{s}(a, b, c)=\int_{a}^{b} \int_{0}^{L_{2}}\left(c+s t_{1}\right)\left(L_{2}-t_{2}\right) g(\rho) \mathrm{d} t_{2} \mathrm{~d} t_{1}
$$

donde:

$$
g(\rho)=\frac{4 K^{2}}{\pi}\left(\frac{L_{3}}{\rho} \mathrm{S}_{0}\left(K \rho, \operatorname{arctg} \frac{L_{3}}{\rho}\right)-\Gamma_{0}\left(K \rho, K \sqrt{L_{3}^{2}+\rho^{2}}\right)\right)
$$

el DEA puede escribirse:

$$
\begin{aligned}
\overline{g_{i} g_{j}} & =I_{1}\left(d-L_{1}^{(i)}, d, L_{1}^{(i)}-d\right)+ \\
& +I_{0}\left(d, d+L_{1}^{(j)}-L_{1}^{(i)}, L_{1}^{(i)}\right)+ \\
& +I_{-1}\left(d-L_{1}^{(j)}-L_{1}^{(i)}, d+L_{1}^{(j)}, d+L_{1}^{(j)}\right)
\end{aligned}
$$

Utilizando el cambio de Ström, $I_{s}(a, b, c)$ puede desarrollarse:

$$
I_{s}(a, b, c)=\int_{a}^{b} \varphi_{1}(\rho) g(\rho) \rho \mathrm{d} \rho+\int_{b}^{\sqrt{a^{2}+L_{2}^{2}}} \varphi_{2}(\rho) g(\rho) \rho \mathrm{d} \rho+\int_{\sqrt{a^{2}+L_{2}^{2}}}^{\sqrt{b^{2}+L_{2}^{2}}} \varphi_{3}(\rho) g(\rho) \rho \mathrm{d} \rho
$$

siendo:

$$
\begin{aligned}
\varphi_{1}(\rho) & =c L_{2} \arccos \frac{a}{\rho}+\frac{s}{2}\left(a^{2}-\rho^{2}\right)+c(a-\rho)+s L_{2} \sqrt{\rho^{2}-a^{2}} \\
\varphi_{2}(\rho) & =c L_{2}\left(\arccos \frac{a}{\rho}-\arccos \frac{b}{\rho}\right)+\frac{s}{2}\left(a^{2}-b^{2}\right)+c(a-b)+ \\
& +s L_{2}\left(\sqrt{\rho^{2}-a^{2}}-\sqrt{\rho^{2}-b^{2}}\right) \\
\varphi_{3}(\rho) & =c L_{2}\left(\operatorname{arcsen} \frac{L_{2}}{\rho}-\arccos \frac{b}{\rho}\right)+\frac{s}{2}\left(\rho^{2}+a^{2}-b^{2}\right)-c b+ \\
& +c \sqrt{\rho^{2}-L_{2}^{2}}-s L_{2} \sqrt{\rho^{2}-b^{2}}
\end{aligned}
$$




\section{A.4.2. Intercambio entre superficies}

\section{A.4.2.1. Intercambio entre superficies planas paralelas}

El DEA entre superficies paralelas (figura 3.5) puede escribirse:

$$
\overline{s_{i} s_{j}}=\int_{0}^{L_{2}} \int_{0}^{L_{2}} \int_{d}^{d+L_{1}^{(j)}} \int_{0}^{L_{1}^{(i)}} \frac{L_{3}^{2}}{\pi r^{4}} \mathrm{e}^{-K r} \mathrm{~d} x_{i} \mathrm{~d} x_{j} \mathrm{~d} y_{i} \mathrm{~d} y_{j}
$$

en la que:

$$
r=\sqrt{\left(x_{j}-x_{i}\right)^{2}+\left(y_{j}-y_{i}\right)^{2}+L_{3}^{2}}
$$

De nuevo, puede utilizarse el cambio de Mohr, para expresar el DEA en la forma:

$$
\begin{aligned}
\overline{s_{i} s_{j}} & =I_{1}\left(d-L_{1}^{(i)}, d, L_{1}^{(i)}-d\right)+ \\
& +I_{0}\left(d, d+L_{1}^{(j)}-L_{1}^{(i)}, L_{1}^{(i)}\right)+ \\
& +I_{-1}\left(d-L_{1}^{(j)}-L_{1}^{(i)}, d+L_{1}^{(j)}, d+L_{1}^{(j)}\right)
\end{aligned}
$$

en la que se ha definido:

$$
I_{s}(a, b, c)=\frac{2 L_{3}^{2}}{\pi} \int_{a}^{b} \int_{0}^{L_{2}}\left(c+s t_{1}\right)\left(L_{2}-t_{2}\right) \frac{\mathrm{e}^{-K \sqrt{t_{1}^{2}+t_{2}^{2}+L_{3}^{2}}}}{\left(t_{1}^{2}+t_{2}^{2}+L_{3}^{2}\right)^{2}} \mathrm{~d} t_{2} \mathrm{~d} t_{1}
$$

Denotando:

$$
g(\rho)=\frac{2 L_{3}^{2}}{\pi} \frac{\mathrm{e}^{-K \sqrt{\rho^{2}+L_{3}^{2}}}}{\left(\rho^{2}+L_{3}^{2}\right)^{2}}
$$

y aplicando el cambio de Ström:

$$
I_{s}(a, b, c)=\int_{a}^{b} \varphi_{1}(\rho) g(\rho) \rho \mathrm{d} \rho+\int_{b}^{\sqrt{a^{2}+L_{2}^{2}}} \varphi_{2}(\rho) g(\rho) \rho \mathrm{d} \rho+\int_{\sqrt{a^{2}+L_{2}^{2}}}^{\sqrt{b^{2}+L_{2}^{2}}} \varphi_{3}(\rho) g(\rho) \rho \mathrm{d} \rho
$$

donde:

$$
\begin{aligned}
\varphi_{1}(\rho) & =c L_{2} \arccos \frac{a}{\rho}+\frac{s}{2}\left(a^{2}-\rho^{2}\right)+c(a-\rho)+s L_{2} \sqrt{\rho^{2}-a^{2}} \\
\varphi_{2}(\rho) & =c L_{2}\left(\arccos \frac{a}{\rho}-\arccos \frac{b}{\rho}\right)+\frac{s}{2}\left(a^{2}-b^{2}\right)+c(a-b)+ \\
& +s L_{2}\left(\sqrt{\rho^{2}-a^{2}}-\sqrt{\rho^{2}-b^{2}}\right) \\
\varphi_{3}(\rho) & =c L_{2}\left(\operatorname{arcsen} \frac{L_{2}}{\rho}-\arccos \frac{b}{\rho}\right)+\frac{s}{2}\left(\rho^{2}+a^{2}-b^{2}\right)-c b+ \\
& +c \sqrt{\rho^{2}-L_{2}^{2}}-s L_{2} \sqrt{\rho^{2}-b^{2}}
\end{aligned}
$$




\section{A.4.2.2. Intercambio entre superficies planas perpendiculares}

El DEA entre superficies perpendiculares (figura 3.6) adopta la forma:

$$
\overline{s_{i} s_{j}}=\int_{0}^{L_{3}} \int_{0}^{L_{2}} \int_{d}^{d+L_{1}^{(j)}} \int_{0}^{L_{1}^{(i)}} \frac{y_{i} z_{j}}{\pi r^{4}} \mathrm{e}^{-K r} \mathrm{~d} x_{i} \mathrm{~d} x_{j} \mathrm{~d} y_{i} \mathrm{~d} z_{j}
$$

donde:

$$
r=\sqrt{\left(x_{j}-x_{i}\right)^{2}+y_{1}^{2}+z_{2}^{2}}
$$

Haciendo el cambio $t_{1}=x_{j}-x_{i}$ :

$$
\begin{aligned}
\overline{s_{i} s_{j}} & =\int_{d-L_{1}^{(i)}}^{d}\left(L_{1}^{(i)}-d+t_{1}\right) f\left(t_{1}\right) \mathrm{d} t_{1}+\int_{d}^{d+L_{1}^{(j)}-L_{1}^{(i)}} L_{1}^{(i)} f\left(t_{1}\right) \mathrm{d} t_{1}+ \\
& +\int_{d+L_{1}^{(j)}-L_{1}^{(i)}}^{d+L_{1}^{(j)}}\left(L_{1}^{(j)}+d-t_{1}\right) f\left(t_{1}\right) \mathrm{d} t_{1}
\end{aligned}
$$

donde:

$$
f\left(t_{1}\right)=\frac{1}{\pi} \int_{0}^{L_{3}} \int_{0}^{L_{2}} y_{i} z_{j} \frac{\mathrm{e}^{-K \sqrt{t_{1}^{2}+y_{1}^{2}+z_{2}^{2}}}}{\left(t_{1}^{2}+y_{1}^{2}+z_{2}^{2}\right)^{2}} \mathrm{~d} y_{i} \mathrm{~d} z_{j}
$$

Para integrar $f\left(t_{1}\right)$ se utilizará una versión alternativa de (A.26):

$$
\int_{0}^{L_{2}} y_{i} \frac{\mathrm{e}^{-K \sqrt{t_{1}^{2}+y_{1}^{2}+z_{2}^{2}}}}{\left(t_{1}^{2}+y_{1}^{2}+z_{2}^{2}\right)^{2}} \mathrm{~d} y_{i}=\frac{\mathrm{E}_{3}\left(K \sqrt{t_{1}^{2}+z_{j}^{2}}\right)}{t_{1}^{2}+z_{j}^{2}}-\frac{\mathrm{E}_{3}\left(K \sqrt{t_{1}^{2}+L_{2}^{2}+z_{j}^{2}}\right)}{t_{1}^{2}+L_{2}^{2}+z_{j}^{2}}
$$

de manera que puede hacerse el siguiente cambio de variables:

$$
\int_{0}^{L_{3}} z_{j} \frac{\mathrm{E}_{3}\left(K \sqrt{t_{1}^{2}+z_{j}^{2}}\right)}{t_{1}^{2}+z_{2}^{2}} \mathrm{~d} z_{j}=\int_{t_{1}}^{\sqrt{t_{1}^{2}+L_{3}^{2}}} \frac{\mathrm{E}_{3}(K r)}{r} \mathrm{~d} r
$$

$\mathrm{y}:$

$$
\int_{0}^{L_{3}} z_{j} \frac{\mathrm{E}_{3}\left(K \sqrt{t_{1}^{2}+L_{2}^{2}+z_{j}^{2}}\right)}{t_{1}^{2}+L_{2}^{2}+z_{j}^{2}} \mathrm{~d} z_{j}=\int_{\sqrt{t_{1}^{2}+L_{2}^{2}}}^{\sqrt{t_{1}^{2}+L_{2}^{2}+L_{3}^{2}}} \frac{\mathrm{E}_{3}(K r)}{r} \mathrm{~d} r
$$

Definiendo:

$$
g(r)=\int \frac{\mathrm{E}_{3}(K r)}{r} \mathrm{~d} r=\frac{1}{2}\left(\mathrm{E}_{3}(K r)+\operatorname{Ei}(-K r)\right)
$$

se tiene:

$$
f\left(t_{1}\right)=\frac{1}{\pi}\left(g\left(\sqrt{t_{1}^{2}+L_{3}^{2}}\right)-g\left(\left|t_{1}\right|\right)+g\left(\sqrt{t_{1}^{2}+L_{2}^{2}}\right)-g\left(\sqrt{t_{1}^{2}+L_{2}^{2}+L_{3}^{2}}\right)\right)
$$




\section{A.4.2.3. Intercambio entre la superficie de una pared final y una superficie plana}

El DEA para este tipo de intercambio (figura 3.7) es:

$$
\overline{s_{i} s_{j}}=\int_{0}^{L_{3}} \int_{0}^{L_{2}} \int_{0}^{L_{2}} \int_{d}^{d+L_{1}} \frac{x_{j} z_{i}}{\pi r^{4}} \mathrm{e}^{-K r} \mathrm{~d} x_{j} \mathrm{~d} y_{i} \mathrm{~d} y_{j} \mathrm{~d} z_{i}
$$

donde:

$$
r=\sqrt{x_{j}^{2}+\left(y_{j}-y_{i}\right)^{2}+z_{i}^{2}}
$$

Haciendo uso del cambio de Mohr:

$$
\overline{s_{i} s_{j}}=\frac{2}{\pi} \int_{0}^{L_{3}} \int_{0}^{L_{2}} \int_{d}^{d+L_{1}}\left(L_{2}-t_{2}\right) x_{j} z_{i} \frac{\mathrm{e}^{-K \sqrt{x_{j}^{2}+t_{2}^{2}+z_{i}^{2}}}}{\left(x_{j}^{2}+t_{2}^{2}+z_{i}^{2}\right)^{2}} \mathrm{~d} x_{j} \mathrm{~d} t_{2} \mathrm{~d} z_{i}
$$

Integrando respecto de $z_{i}$ :

$$
\overline{s_{i} s_{j}}=\frac{2 K^{2}}{\pi} \int_{0}^{L_{2}} \int_{d}^{d+L_{1}} x_{j}\left(L_{2}-t_{2}\right) \Gamma_{-2}\left(K \rho, K \sqrt{\rho^{2}+L_{3}^{2}}\right) \mathrm{d} x_{j} \mathrm{~d} t_{2}
$$

donde:

$$
\rho=\rho\left(x_{j}, t_{2}\right)=\sqrt{x_{j}^{2}+t_{2}^{2}}
$$

Definiendo:

$$
g(\rho)=\frac{2 K^{2}}{\pi} \Gamma_{-2}\left(K \rho, K \sqrt{\rho^{2}+L_{3}^{2}}\right)
$$

y aplicando el cambio de Ström, el DEA queda finalmente:

$$
\overline{s_{i} s_{j}}=\int_{d}^{d+L_{1}} \varphi_{1}(\rho) g(\rho) \rho \mathrm{d} \rho+\int_{d+L_{1}}^{\sqrt{d^{2}+L_{2}^{2}}} \varphi_{2}(\rho) g(\rho) \rho \mathrm{d} \rho+\int_{\sqrt{d^{2}+L_{2}^{2}}}^{\sqrt{\left(d+L_{1}\right)^{2}+L_{2}^{2}}} \varphi_{3}(\rho) g(\rho) \rho \mathrm{d} \rho
$$

siendo:

$$
\begin{aligned}
& \varphi_{1}(\rho)=\frac{1}{2}\left(d^{2}-\rho^{2}\right)+L_{2} \sqrt{\rho^{2}-d^{2}} \\
& \varphi_{2}(\rho)=\frac{1}{2}\left(d^{2}-\left(d+L_{1}\right)^{2}\right)+L_{2}\left(\sqrt{\rho^{2}-d^{2}}-\sqrt{\rho^{2}-\left(d+L_{1}\right)^{2}}\right) \\
& \varphi_{3}(\rho)=\frac{1}{2}\left(\rho^{2}+L_{2}^{2}-\left(d+L_{1}\right)^{2}\right)-L_{2} \sqrt{\rho^{2}-\left(d+L_{1}\right)^{2}}
\end{aligned}
$$




\section{A.4.2.4. Intercambio entre las superficies de las paredes finales}

El intercambio directo entre las superficies de las paredes finales (figura 3.8) viene dado por:

$$
\overline{s_{i} s_{j}}=\int_{0}^{L_{3}} \int_{0}^{L_{3}} \int_{0}^{L_{2}} \int_{0}^{L_{2}} \frac{d^{2}}{\pi r^{4}} \mathrm{e}^{-K r} \mathrm{~d} y_{i} \mathrm{~d} y_{j} \mathrm{~d} z_{i} \mathrm{~d} z_{j}
$$

donde:

$$
r=\sqrt{d^{2}+\left(y_{j}-y_{i}\right)^{2}+\left(z_{j}-z_{i}\right)^{2}}
$$

Haciendo el ya habitual cambio de Mohr se obtiene:

$$
\overline{s_{i} s_{j}}=\frac{4 d^{2}}{\pi} \int_{0}^{L_{3}} \int_{0}^{L_{2}}\left(L_{2}-t_{2}\right)\left(L_{3}-t_{3}\right) \frac{\mathrm{e}^{-K \sqrt{d^{2}+t_{2}^{2}+t_{3}^{2}}}}{\left(d^{2}+t_{2}^{2}+t_{3}^{2}\right)^{2}} \mathrm{~d} t_{2} \mathrm{~d} t_{3}
$$

Se define:

$$
g(\rho)=\frac{4 d^{2}}{\pi} \frac{\mathrm{e}^{-K \sqrt{d^{2}+\rho^{2}}}}{\left(d^{2}+\rho^{2}\right)^{2}}
$$

donde:

$$
\rho=\rho\left(t_{2}, t_{3}\right)=\sqrt{t_{2}^{2}+t_{3}^{2}}
$$

Haciendo el cambio de Ström:

$$
\overline{s_{i} s_{j}}=\int_{0}^{L_{2}} \varphi_{1}(\rho) g(\rho) \rho \mathrm{d} \rho+\int_{L_{2}}^{L_{3}} \varphi_{2}(\rho) g(\rho) \rho \mathrm{d} \rho+\int_{L_{3}}^{\sqrt{L_{2}^{2}+L_{3}^{2}}} \varphi_{3}(\rho) g(\rho) \rho \mathrm{d} \rho
$$

siendo:

$$
\begin{aligned}
\varphi_{1}(\rho) & =\frac{\pi}{2} L_{2} L_{3}+\frac{\rho^{2}}{2}-\rho\left(L_{2}-L_{3}\right) \\
\varphi_{2}(\rho) & =L_{2} L_{3} \operatorname{arcsen} \frac{L_{2}}{\rho}-\frac{L_{2}^{2}}{2}-\rho L_{3}+L_{3} \sqrt{\rho^{2}-L_{2}^{2}} \\
\varphi_{3}(\rho) & =L_{2} L_{3}\left(\operatorname{arcsen} \frac{L_{2}}{\rho}-\arccos \frac{b}{\rho}\right)-\frac{1}{2}\left(\rho^{2}+L_{2}^{2}+L_{3}^{2}\right)+ \\
& +L_{2} \sqrt{\rho^{2}-L_{3}^{2}}+L_{3} \sqrt{\rho^{2}-L_{2}^{2}}
\end{aligned}
$$


A.4.2.5. Intercambio entre la superficie de la solera y la superficie de las baldosas en contacto con los rodillos

El intercambio directo entre la solera y las baldosas se produce a través de los huecos entre dos rodillos sucesivos (figura 3.9):

$$
\overline{s_{i} s_{j}}= \begin{cases}2\left(\frac{L_{1}}{\delta}-\left\lfloor\frac{x_{i}^{+}}{\delta}+\frac{1}{2}\right\rfloor\right) I_{\infty}+2 \sum_{k=1}^{\frac{x_{i}^{+}}{\delta}+\frac{1}{2}} I_{k} & \text { si están en el mismo sector } \\ \left\lfloor\frac{x_{i}^{+}}{\delta}+\frac{1}{2}\right\rfloor I_{\infty}-\sum_{k=1}^{\frac{x_{i}^{+}}{\delta}+\frac{1}{2}} I_{k} & \text { si están en sectores contiguos } \\ 0 & \text { en caso contrario }\end{cases}
$$

siendo:

$$
\begin{aligned}
& I_{\infty}=\int_{0}^{L_{2}} \int_{0}^{L_{2}} \int_{0}^{x_{i}^{+}} \int_{x_{j}^{-}\left(x_{i}\right)}^{x_{j}^{+}\left(x_{i}\right)} \frac{L_{3}^{2}}{\pi r^{4}} \mathrm{e}^{-K r} \mathrm{~d} x_{j} \mathrm{~d} x_{i} \mathrm{~d} y_{i} \mathrm{~d} y_{j} \\
& I_{k}=\int_{0}^{L_{2}} \int_{0}^{L_{2}} \int_{0}^{\left(k+\frac{1}{2}\right) \delta} \int_{x_{j}^{-}\left(x_{i}\right)}^{x_{j}^{+}\left(x_{i}\right)} \frac{L_{3}^{2}}{\pi r^{4}} \mathrm{e}^{-K r} \mathrm{~d} x_{j} \mathrm{~d} x_{i} \mathrm{~d} t_{2} \\
& r=\sqrt{\left(x_{j}-x_{i}\right)^{2}+\left(y_{j}-y_{i}\right)^{2}+L_{3}^{2}} \\
& x_{i}^{+}=\left(L_{3}-R\right) \sqrt{\left(\frac{\delta}{2 R}\right)^{2}-1} \\
& x_{j}^{-}\left(x_{i}\right)=x_{i}-\left(L_{3}-R\right) \frac{x_{i}+\frac{\delta}{2}-R \sqrt{1-\frac{R^{2}-\left(x_{i}+\frac{\delta}{2}\right)^{2}}{\left(L_{3}-R\right)^{2}}}}{L_{3}-2 R} \\
& x_{j}^{+}\left(x_{i}\right)=x_{i}-\left(L_{3}-R\right) \frac{x_{i}-\frac{\delta}{2}+R \sqrt{1-\frac{R^{2}-\left(x_{i}-\frac{\delta}{2}\right)^{2}}{\left(L_{3}-R\right)^{2}}}}{L_{3}-2 R}
\end{aligned}
$$

Utilizando el cambio de Mohr $t_{2}=y_{j}-y_{i}$ :

$$
\begin{gathered}
I_{\infty}=L_{3} \int_{0}^{x_{i}^{+}} \int_{x_{j}^{-}\left(x_{i}\right)}^{x_{j}^{+}\left(x_{i}\right)} g(\rho) \mathrm{d} x_{j} \mathrm{~d} x_{i} \\
I_{k}=L_{3} \int_{0}^{\left(k+\frac{1}{2}\right) \delta} \int_{x_{j}^{-}\left(x_{i}\right)}^{x_{j}^{+}\left(x_{i}\right)} g(\rho) \mathrm{d} x_{j} \mathrm{~d} x_{i}
\end{gathered}
$$


donde:

$$
g(\rho)=\frac{2 L_{3}}{\pi} \int_{0}^{L_{2}}\left(L_{2}-t_{2}\right) \frac{\mathrm{e}^{-K \sqrt{t_{2}^{2}+\rho^{2}}}}{\left(t_{2}^{2}+\rho^{2}\right)^{2}} \mathrm{~d} t_{2}
$$

en la que:

$$
\rho=\rho\left(x_{i}, x_{j}\right)=\sqrt{\left(x_{j}-x_{i}\right)^{2}+L_{3}^{2}}
$$

Haciendo uso de las expresiones recogidas en $§ \mathrm{~A} .3$ :

$$
\begin{aligned}
g(\rho) & =\frac{L_{2}^{2} L_{3}}{\pi \rho^{2}}\left(\frac{\mathrm{e}^{-K \sqrt{t_{2}^{2}+\rho^{2}}}}{L_{2}^{2}+\rho^{2}}-K \frac{\mathrm{e}^{-K \sqrt{t_{2}^{2}+\rho^{2}}}}{\sqrt{t_{2}^{2}+\rho^{2}}}\right)+ \\
& +\frac{L_{2} L_{3}}{\pi \rho^{3}}\left(\left(1+K^{2} \rho^{2}\right) \mathrm{S}_{0}\left(K \rho, \operatorname{arctg} \frac{L_{2}}{\rho}\right)+\mathrm{S}_{1}\left(K \rho, \operatorname{arctg} \frac{L_{2}}{\rho}\right)-\right. \\
& \left.-\mathrm{S}_{2}\left(K \rho, \operatorname{arctg} \frac{L_{2}}{\rho}\right)\right)-\frac{2 L_{3} K^{2}}{\pi} \Gamma_{-2}\left(K \rho, K \sqrt{L_{2}^{2}+\rho^{2}}\right)
\end{aligned}
$$

Puede aplicarse una vez más el cambio de Mohr, haciendo $t_{1}=x_{j}-x_{i}$, pero en este caso los límites de integración son más complicados que los que aparecen en la figura A.1:

$$
\begin{aligned}
& t_{1}^{-}\left(x_{i}\right)=-\left(L_{3}-R\right) \frac{x_{i}+\frac{\delta}{2}-R \sqrt{1-\frac{R^{2}-\left(x_{i}+\frac{\delta}{2}\right)^{2}}{\left(L_{3}-R\right)^{2}}}}{L_{3}-2 R} \\
& t_{1}^{+}\left(x_{i}\right)=-\left(L_{3}-R\right) \frac{x_{i}-\frac{\delta}{2}+R \sqrt{1-\frac{R^{2}-\left(x_{i}-\frac{\delta}{2}\right)^{2}}{\left(L_{3}-R\right)^{2}}}}{L_{3}-2 R}
\end{aligned}
$$

Tras la aplicación de dicho cambio de variables, se obtiene:

$$
\begin{aligned}
I_{\infty} & =\int_{t_{1}^{-}\left(x_{i}^{+}\right)}^{t_{1}^{+}\left(x_{i}^{+}\right)}\left(L_{3}\left(x_{i}^{+}+\frac{\delta}{2}\right)+\left(L_{3}-R\right) t_{1}-R \rho\right) g(\rho) \mathrm{d} t_{1}+ \\
& +\int_{t_{1}^{+}\left(x_{i}^{+}\right)}^{t_{1}^{-}(0)}\left(L_{3} \delta-2 R \rho\right) g(\rho) \mathrm{d} t_{1}+\int_{t_{1}^{-}(0)}^{t_{1}^{+}(0)}\left(L_{3} \frac{\delta}{2}-\left(L_{3}-R\right) t_{1}-R \rho\right) g(\rho) \mathrm{d} t_{1} \\
I_{k}= & \int_{t_{1}^{+}\left(\left(k-\frac{1}{2}\right) \delta\right)}\left(k L_{3} \delta+\left(L_{3}-R\right) t_{1}-R \rho\right) g(\rho) \mathrm{d} t_{1}+ \\
& \int_{t_{1}^{-}(0)}^{+}\left(L_{3} \delta-2 R \rho\right) g(\rho) \mathrm{d} t_{1}+\int_{t_{1}^{-}(0)}^{t_{1}^{+}(0)}\left(L_{3} \frac{\delta}{2}-\left(L_{3}-R\right) t_{1}-R \rho\right) g(\rho) \mathrm{d} t_{1}
\end{aligned}
$$

siendo:

$$
\rho=\rho\left(t_{1}\right)=\sqrt{t_{1}^{2}+L_{3}^{2}}
$$




\section{A.4.2.6. Intercambio entre la superficie de las paredes laterales y la superficie de las baldosas en contacto con los rodillos}

El intercambio entre las paredes laterales inferiores y la baldosas (figura 3.10) puede calcularse mediante:

$$
\overline{s_{i} s_{j}}= \begin{cases}2\left(\frac{L_{1}}{\delta}-\left\lfloor\frac{x_{i}^{+}}{\delta}+\frac{1}{2}\right\rfloor\right) I_{\infty}+2 \sum_{k=1}^{\frac{x_{i}^{+}}{\delta}+\frac{1}{2}} I_{k} & \text { si están en el mismo sector } \\ \left.\frac{x_{i}^{+}}{\delta}+\frac{1}{2}\right\rfloor I_{\infty}-\sum_{k=1}^{\frac{x_{i}^{+}}{\delta}+\frac{1}{2}} I_{k} & \text { si están en sectores contiguos } \\ 0 & \text { en caso contrario }\end{cases}
$$

donde:

$$
\begin{aligned}
& I_{\infty}=\int_{0}^{d} \int_{0}^{L_{2}} \int_{0}^{x_{i}^{+}\left(z_{i}\right)} \int_{x_{j}^{-}\left(x_{i}, z_{i}\right)}^{x_{j}^{+}\left(x_{i}, z_{i}\right)} \frac{y_{j}\left(L_{3}-z_{i}\right)}{\pi r^{4}} \mathrm{e}^{-K r} \mathrm{~d} x_{j} \mathrm{~d} x_{i} \mathrm{~d} y_{j} \mathrm{~d} z_{i} \\
& I_{k}=\int_{0}^{d} \int_{0}^{L_{2}} \int_{0}^{\left(k+\frac{1}{2}\right) \delta} \int_{x_{j}^{-}\left(x_{i}, z_{i}\right)}^{x_{j}^{+}\left(x_{i}, z_{i}\right)} \frac{y_{j}\left(L_{3}-z_{i}\right)}{\pi r^{4}} \mathrm{e}^{-K r} \mathrm{~d} x_{j} \mathrm{~d} x_{i} \mathrm{~d} y_{j} \mathrm{~d} z_{i} \\
& r=\sqrt{\left(x_{j}-x_{i}\right)^{2}+y_{j}^{2}+\left(L_{3}-z_{i}\right)^{2}} \\
& x_{i}^{+}\left(z_{i}\right)=\left(L_{3}-R-z_{i}\right) \sqrt{\left(\frac{\delta}{2 R}\right)^{2}-1} \\
& x_{j}^{-}\left(x_{i}, z_{i}\right)=x_{i}-\left(L_{3}-R-z_{i}\right) \frac{x_{i}+\frac{\delta}{2}-R \sqrt{1-\frac{R^{2}-\left(x_{i}+\frac{\delta}{2}\right)^{2}}{\left(L_{3}-R-z_{i}\right)^{2}}}}{L_{3}-2 R-z_{i}} \\
& x_{j}^{+}\left(x_{i}, z_{i}\right)=x_{i}-\left(L_{3}-R-z_{i}\right) \frac{x_{i}-\frac{\delta}{2}+R \sqrt{1-\frac{R^{2}-\left(x_{i}-\frac{\delta}{2}\right)^{2}}{\left(L_{3}-R-z_{i}\right)^{2}}}}{L_{3}-2 R-z_{i}}
\end{aligned}
$$

Integrando respecto de $y_{j}$ :

$$
\begin{aligned}
I_{\infty} & =\int_{0}^{d} \int_{0}^{x_{i}^{+}\left(z_{i}\right)} \int_{x_{j}^{-}\left(x_{i}, z_{i}\right)}^{x_{j}^{+}\left(x_{i}, z_{i}\right)}\left(L_{3}-z_{i}\right) g(\rho) \mathrm{d} x_{j} \mathrm{~d} x_{i} \mathrm{~d} z_{i} \\
I_{k} & =\int_{0}^{d} \int_{0}^{\left(k+\frac{1}{2}\right) \delta} \int_{x_{j}^{-}\left(x_{i}, z_{i}\right)}^{x_{j}^{+}\left(x_{i}, z_{i}\right)}\left(L_{3}-z_{i}\right) g(\rho) \mathrm{d} x_{j} \mathrm{~d} x_{i} \mathrm{~d} z_{i}
\end{aligned}
$$


en las que:

$$
g(\rho)=\frac{K^{2}}{\pi} \Gamma_{-2}\left(K \rho, K \sqrt{L_{2}+\rho^{2}}\right)
$$

donde:

$$
\rho=\rho\left(x_{i}, x_{j}, z_{i}\right)=\sqrt{\left(x_{j}-x_{i}\right)^{2}+\left(L_{3}-z_{i}\right)^{2}}
$$

Utilizando el cambio $t_{1}=x_{j}-x_{i}$ y definiendo:

$$
\begin{aligned}
t_{1}^{-}\left(x_{i}, z_{i}\right) & =-\left(L_{3}-R-z_{i}\right) \frac{x_{i}+\frac{\delta}{2}-R \sqrt{1-\frac{R^{2}-\left(x_{i}+\frac{\delta}{2}\right)^{2}}{\left(L_{3}-R-z_{i}\right)^{2}}}}{L_{3}-2 R-z_{i}} \\
t_{1}^{+}\left(x_{i}, z_{i}\right) & =-\left(L_{3}-R-z_{i}\right) \frac{x_{i}-\frac{\delta}{2}+R \sqrt{1-\frac{R^{2}-\left(x_{i}-\frac{\delta}{2}\right)^{2}}{\left(L_{3}-R-z_{i}\right)^{2}}}}{L_{3}-2 R-z_{i}}
\end{aligned}
$$

se llega a:

$$
\begin{aligned}
& I_{\infty}=\int_{0}^{L_{3}-R} \int_{t_{1}^{-}\left(x_{i}^{+}, z_{i}\right)}^{t_{1}^{+}\left(x_{i}^{+}, z_{i}\right)}\left(\left(L_{3}-z_{i}\right)\left(x_{i}^{+}+\frac{\delta}{2}\right)+\left(L_{3}-R-z_{i}\right) t_{1}-R \rho\right) g(\rho) \mathrm{d} t_{1} \mathrm{~d} z_{i}+ \\
& +\int_{0}^{L_{3}-R} \int_{t_{1}^{+}\left(x_{i}^{+}, z_{i}\right)}^{t_{1}^{-}\left(0, z_{i}\right)}\left(\left(L_{3}-z_{i}\right) \delta-2 R \rho\right) g(\rho) \mathrm{d} t_{1} \mathrm{~d} z_{i}+ \\
& +\int_{0}^{L_{3}-R} \int_{t_{1}^{-}\left(0, z_{i}\right)}^{t_{1}^{+}\left(0, z_{i}\right)}\left(\left(L_{3}-z_{i}\right) \frac{\delta}{2}-\left(L_{3}-R-z_{i}\right) t_{1}-R \rho\right) g(\rho) \mathrm{d} t_{1} \mathrm{~d} z_{i} \\
& I_{k}=\int_{0}^{L_{3}-R} \int_{t_{1}^{-}\left(\left(k-\frac{1}{2}\right) \delta, z_{i}\right)}^{t_{1}^{+}\left(\left(k-\frac{1}{2}\right) \delta, z_{i}\right)}\left(k\left(L_{3}-z_{i}\right) \delta+\left(L_{3}-R-z_{i}\right) t_{1}-R \rho\right) g(\rho) \mathrm{d} t_{1} \mathrm{~d} z_{i}+ \\
& +\int_{0}^{L_{3}-R} \int_{t_{1}^{+}\left(\left(k-\frac{1}{2}\right) \delta, z_{i}\right)}^{t_{1}^{-}\left(0, z_{i}\right)}\left(\left(L_{3}-z_{i}\right) \delta-2 R \rho\right) g(\rho) \mathrm{d} t_{1} \mathrm{~d} z_{i}+ \\
& +\int_{0}^{L_{3}-R} \int_{t_{1}^{-}\left(0, z_{i}\right)}^{t_{1}^{+}}\left(\left(L_{3}-z_{i}\right) \frac{\delta}{2}-\left(L_{3}-R-z_{i}\right) t_{1}-R \rho\right) g(\rho) \mathrm{d} t_{1} \mathrm{~d} z_{i}
\end{aligned}
$$

siendo:

$$
\rho=\rho\left(t_{1}, z_{i}\right)=\sqrt{t_{1}^{2}+\left(L_{3}-z_{i}\right)^{2}}
$$


A.4.2.7. Intercambio entre la superficie de una pared final y la superficie de las baldosas en contacto con los rodillos

El intercambio directo entre las paredes finales inferiores y la baldosas (figura 3.11) puede calcularse mediante:

$$
\overline{s_{i} s_{j}}= \begin{cases}\sum_{k=1}^{\frac{x_{i}^{+}}{\delta}+\frac{1}{2}} I_{k} & \text { si están en el mismo sector } \\ 0 & \text { en caso contrario }\end{cases}
$$

donde:

$$
\begin{aligned}
& I_{k}=\int_{0}^{z_{i}^{+}} \int_{0}^{L_{2}} \int_{0}^{L_{2}} \int_{x_{j}^{-}\left(x_{i}\right)}^{x_{j}^{+}\left(x_{i}\right)} \frac{\left(x_{j}-\left(k-\frac{1}{2}\right) \delta\right)\left(L_{3}-z_{i}\right)}{\pi r^{4}} \mathrm{e}^{-K r} \mathrm{~d} x_{j} \mathrm{~d} y_{i} \mathrm{~d} y_{j} \mathrm{~d} z_{i} \\
& r=\sqrt{\left(x_{j}-\left(k+\frac{1}{2}\right) \delta\right)^{2}+\left(y_{j}-y_{i}\right)^{2}+\left(L_{3}-z_{i}\right)^{2}} \\
& x_{i}^{+}=\left(L_{3}-R\right) \sqrt{\left(\frac{\delta}{2 R}\right)^{2}-1} \\
& z_{i}^{+}=L_{3}-R-\frac{\left(k-\frac{1}{2}\right) \delta}{\sqrt{\left(\frac{\delta}{2 R}\right)^{2}-1}} \\
& x_{j}^{-}\left(z_{i}\right)=\left(k+\frac{1}{2}\right) \delta-\left(L_{3}-R-z_{i}\right) \frac{k \delta-R \sqrt{1-\frac{R^{2}-k^{2} \delta^{2}}{\left(L_{3}-R-z_{i}\right)^{2}}}}{L_{3}-2 R-z_{i}} \\
& x_{j}^{+}\left(z_{i}\right)=\left(k+\frac{1}{2}\right) \delta-\left(L_{3}-R-z_{i}\right) \frac{(k-1) \delta+R \sqrt{1-\frac{R^{2}-(k-1)^{2} \delta^{2}}{\left(L_{3}-R-z_{i}\right)^{2}}}}{L_{3}-2 R-z_{i}}
\end{aligned}
$$

Haciendo $t_{2}=y_{j}-y_{i}$ y $t_{1}=x_{j}-\left(k-\frac{1}{2}\right) \delta$ :

$$
I_{k}=\frac{2}{\pi} \int_{0}^{z_{i}^{+}} \int_{0}^{L_{2}} \int_{t_{1}^{-}\left(x_{i}\right)}^{t_{1}^{+}\left(x_{i}\right)} t_{1}\left(L_{2}-t_{2}\right)\left(L_{3}-z_{i}\right) \frac{\mathrm{e}^{-K \sqrt{t_{1}^{2}+t_{2}^{2}+\left(L_{3}-z_{i}\right)^{2}}}}{\left(t_{1}^{2}+t_{2}^{2}+\left(L_{3}-z_{i}\right)^{2}\right)^{2}} \mathrm{~d} t_{1} \mathrm{~d} t_{2} \mathrm{~d} z_{i}
$$

donde:

$$
\begin{aligned}
& t_{1}^{-}\left(z_{i}\right)=-\left(L_{3}-R-z_{i}\right) \frac{k \delta-R \sqrt{1-\frac{R^{2}-k^{2} \delta^{2}}{\left(L_{3}-R-z_{i}\right)^{2}}}}{L_{3}-2 R-z_{i}} \\
& t_{1}^{+}\left(z_{i}\right)=-\left(L_{3}-R-z_{i}\right) \frac{(k-1) \delta+R \sqrt{1-\frac{R^{2}-(k-1)^{2} \delta^{2}}{\left(L_{3}-R-z_{i}\right)^{2}}}}{L_{3}-2 R-z_{i}}
\end{aligned}
$$


Integrando respecto de $t_{1}$ :

$$
I_{k}=2 \int_{0}^{z_{i}^{+}} \int_{0}^{L_{2}}\left(L_{2}-t_{2}\right)\left(L_{3}-z_{i}\right) g\left(\rho, z_{i}\right) \mathrm{d} t_{2} \mathrm{~d} z_{i}
$$

donde:

$$
g\left(\rho, z_{i}\right)=\frac{2 K^{2}}{\pi} \Gamma_{-2}\left(K \sqrt{t_{1}^{+}\left(z_{i}\right)^{2}+\rho^{2}}, K \sqrt{t_{1}^{-}\left(z_{i}\right)^{2}+\rho^{2}}\right)
$$

$\mathrm{y}$ :

$$
\rho=\rho\left(t_{2}, z_{i}\right)=\sqrt{t_{2}^{2}+\left(L_{3}-z_{i}\right)^{2}}
$$

\section{A.4.2.8. Intercambio entre la superficie de las baldosas en contacto con los rodillos y la superficie de los rodillos}

Las baldosas solamente pueden intercambiar directamente energía radiante con los rodillos en su mismo sector (figura 3.12). La longitud de los rodillos es mucho mayor que su radio y que la distancia de separación entre ellos. Puede considerarse pues que la longitud de los rodillos es infinita en comparación con las otras dimensiones. Bajo esta consideración, el DEA entre las baldosas y los rodillos puede calcularse con:

$$
\overline{s_{i} s_{j}}=\frac{L_{1} L_{2}}{\delta} \int_{-\infty}^{\infty} \int_{0}^{\delta} \int_{\alpha_{j}^{-}\left(x_{i}\right)}^{3 \pi / 2} \frac{\cos \theta_{i} \cos \theta_{j}}{\pi r^{2}} \mathrm{e}^{-K r} R \mathrm{~d} \alpha_{j} \mathrm{~d} x_{i} \mathrm{~d} t_{2}
$$

siendo:

$$
\begin{aligned}
& r=\sqrt{\left(\delta+R \cos \alpha_{j}-x_{i}\right)^{2}+\left(y_{j}-y_{i}\right)^{2}+R^{2}\left(1+\operatorname{sen} \alpha_{j}\right)^{2}} \\
& \cos \theta_{i}=\frac{R\left(1+\operatorname{sen} \alpha_{j}\right)}{r} \\
& \cos \theta_{j}=\frac{\left(x_{i}-\delta\right) \cos \alpha_{j}-R\left(1+\operatorname{sen} \alpha_{j}\right)}{r} \\
& \alpha_{j}^{-}\left(x_{i}\right)= \begin{cases}2 \operatorname{arctg} \frac{x_{i}^{2}-R^{2}-2 \sqrt{\delta x_{i}\left(R^{2}+x_{i}\left(x_{i}-\delta\right)\right)}}{\left(x_{i}+R\right)^{2}-2 \delta x_{i}} & \text { si } x_{i}<\frac{\delta}{2}-\frac{\delta}{2} \sqrt{1-\left(\frac{2 R}{\delta}\right)^{2}} \\
\operatorname{arctg} \frac{\left(x_{i}-\delta\right)^{2}-R^{2}}{2 R\left(x_{i}-\delta\right)} & \text { si } x_{i} \geq \frac{\delta}{2}-\frac{\delta}{2} \sqrt{1-\left(\frac{2 R}{\delta}\right)^{2}}\end{cases}
\end{aligned}
$$

El DEA puede reescribirse:

$$
\overline{s_{i} s_{j}}=\frac{L_{1} L_{2} R}{\pi \delta} \int_{0}^{\delta} \int_{\alpha_{j}^{-}\left(x_{i}\right)}^{3 \pi / 2} f\left(x_{i}, \alpha_{j}\right) g(\rho) \mathrm{d} \alpha_{j} \mathrm{~d} x_{i}
$$


donde:

$$
f\left(x_{i}, \alpha_{j}\right)=R\left(1+\operatorname{sen} \alpha_{j}\right)\left(\left(x_{i}-\delta\right) \cos \alpha_{j}-R\left(1+\operatorname{sen} \alpha_{j}\right)\right)
$$

y:

$$
g(\rho)=\int_{-\infty}^{\infty} \frac{\mathrm{e}^{-K \sqrt{y^{2}+\rho^{2}}}}{\left(t_{2}^{2}+\rho^{2}\right)^{2}} \mathrm{~d} t_{2}
$$

en la que:

$$
\rho=\rho\left(x_{i}, \alpha_{j}\right)=\sqrt{\left(\delta+R \cos \alpha_{j}-x_{i}\right)^{2}+R^{2}\left(1+\operatorname{sen} \alpha_{j}\right)^{2}}
$$

Evaluando $g(\rho)$ :

$$
g(\rho)=\frac{1}{\rho^{3}}\left(\left(1+K^{2} \rho^{2}\right) \mathrm{S}_{0}\left(K \rho, \frac{\pi}{2}\right)+\mathrm{S}_{1}\left(K \rho, \frac{\pi}{2}\right)-\mathrm{S}_{2}\left(K \rho, \frac{\pi}{2}\right)\right)
$$

\section{A.4.2.9. Intercambio entre las superficies de los rodillos}

El DEA entre las superficies de los rodillos (figura 3.13) guarda similitudes con el DEA entre las baldosas y los rodillos. Al igual que en dicho caso, puede considerarse que la longitud de los rodillos es infinita:

$$
\overline{s_{i} s_{j}}=\frac{4 L_{1} L_{2}}{\delta} \int_{-\infty}^{\infty} \int_{0}^{\frac{\pi}{2}} \int_{\alpha_{j}^{-}\left(\alpha_{i}\right)}^{\alpha_{j}^{+}\left(\alpha_{i}\right)} \frac{\cos \theta_{i} \cos \theta_{j}}{\pi r^{2}} \mathrm{e}^{-K r} R^{2} \mathrm{~d} \alpha_{j} \mathrm{~d} \alpha_{i} \mathrm{~d} t_{2}
$$

donde:

$$
\begin{aligned}
& r=\sqrt{\left(\delta+R \cos \alpha_{j}-R \cos \alpha_{i}\right)^{2}+\left(y_{j}-y_{i}\right)^{2}+R^{2}\left(\operatorname{sen} \alpha_{j}-\operatorname{sen} \alpha_{i}\right)^{2}} \\
& \cos \theta_{i}=\frac{R\left(\cos \left(\alpha_{j}-\alpha_{i}\right)-1\right)+\delta \cos \alpha_{i}}{r} \\
& \cos \theta_{j}=\frac{R\left(\cos \left(\alpha_{j}-\alpha_{i}\right)-1\right)+\delta \cos \alpha_{j}}{r} \\
& \alpha_{j}^{-}\left(\alpha_{i}\right)=2 \operatorname{arctg} \frac{R \operatorname{sen} \alpha_{i}-\sqrt{\delta^{2}-2 R \delta \cos \alpha_{i}}}{R\left(1+\cos \alpha_{i}\right)-\delta} \\
& \alpha_{j}^{+}\left(\alpha_{i}\right)= \begin{cases}2 \operatorname{arctg} \frac{R \operatorname{sen} \alpha_{i}+\sqrt{\delta^{2}-2 R \delta \cos \alpha_{i}}}{R\left(1+\cos \alpha_{i}\right)-\delta} & \text { si } \alpha_{i} \leq \arccos \frac{2 R}{\delta} \\
2 \operatorname{arctg} \frac{R \operatorname{sen} \alpha_{i}+\sqrt{2 R \delta \cos \alpha_{i}-\delta^{2} \cos ^{2} \alpha_{i}}}{R\left(1+\cos \alpha_{i}\right)-\delta \cos \alpha_{i}} & \text { si } \alpha_{i}>\arccos \frac{2 R}{\delta}\end{cases}
\end{aligned}
$$


El DEA puede reescribirse:

$$
\overline{s_{i} s_{j}}=\frac{4 L_{1} L_{2} R^{2}}{\pi \delta} \int_{0}^{\frac{\pi}{2}} \int_{\alpha_{j}^{-}\left(\alpha_{i}\right)}^{\alpha_{j}^{+}\left(\alpha_{i}\right)} f\left(\alpha_{i}, \alpha_{j}\right) g(\rho) \mathrm{d} \alpha_{j} \mathrm{~d} \alpha_{i}
$$

donde:

$$
f\left(\alpha_{i}, \alpha_{j}\right)=\left(R\left(\cos \left(\alpha_{j}-\alpha_{i}\right)-1\right)+\delta \cos \alpha_{i}\right)\left(R\left(\cos \left(\alpha_{j}-\alpha_{i}\right)-1\right)+\delta \cos \alpha_{j}\right)
$$

y:

$$
g(\rho)=\int_{-\infty}^{\infty} \frac{\mathrm{e}^{-K \sqrt{y^{2}+\rho^{2}}}}{\left(t_{2}^{2}+\rho^{2}\right)^{2}} \mathrm{~d} t_{2}
$$

en la que:

$$
\rho=\rho\left(\alpha_{i}, \alpha_{j}\right)=\sqrt{\left(\delta+R\left(\cos \alpha_{j}-\cos \alpha_{i}\right)\right)^{2}+R^{2}\left(\operatorname{sen} \alpha_{j}-\operatorname{sen} \alpha_{i}\right)^{2}}
$$

La versión integrada de $g(\rho)$ ha aparecido anteriormente:

$$
g(\rho)=\frac{1}{\rho^{3}}\left(\left(1+K^{2} \rho^{2}\right) \mathrm{S}_{0}\left(K \rho, \frac{\pi}{2}\right)+\mathrm{S}_{1}\left(K \rho, \frac{\pi}{2}\right)-\mathrm{S}_{2}\left(K \rho, \frac{\pi}{2}\right)\right)
$$

\section{A.4.3. Intercambio entre volúmenes de gas y superficies}

\section{A.4.3.1. Intercambio entre un volumen de gas y una superficie plana}

El DEA entre un volumen de gas y una superficie plana, dispuestas según la configuración de la (figura 3.15), puede escribirse:

$$
\overline{g_{i} s_{j}}=\int_{0}^{L_{3}} \int_{0}^{L_{2}} \int_{0}^{L_{2}} \int_{d}^{d+L_{1}^{(j)}} \int_{0}^{L_{1}^{(i)}} \frac{K z_{i}}{\pi r^{3}} \mathrm{e}^{-K r} \mathrm{~d} x_{i} \mathrm{~d} x_{j} \mathrm{~d} y_{i} \mathrm{~d} y_{j} \mathrm{~d} z_{i}
$$

donde:

$$
r=\sqrt{\left(x_{j}-x_{i}\right)^{2}+\left(y_{j}-y_{i}\right)^{2}+z_{i}^{2}}
$$

Por aplicación del cambio de Mohr:

$$
\begin{aligned}
\overline{g_{i} s_{j}} & =\int_{d-L_{1}^{(i)}}^{d}\left(L_{1}^{(i)}-d+t_{1}\right) f\left(t_{1}\right) \mathrm{d} t_{1}+\int_{d}^{d+L_{1}^{(j)}-L_{1}^{(i)}} L_{1}^{(i)} f\left(t_{1}\right) \mathrm{d} t_{1}+ \\
& +\int_{d+L_{1}^{(j)}-L_{1}^{(i)}}^{d+L_{1}^{(j)}}\left(d+L_{1}^{(j)}-t_{1}\right) f\left(t_{1}\right) \mathrm{d} t_{1}
\end{aligned}
$$


en la que:

$$
f\left(t_{1}\right)=\frac{2 K}{\pi} \int_{0}^{L_{3}} \int_{0}^{L_{2}}\left(L_{2}-t_{2}\right) z_{i} \frac{\mathrm{e}^{-K \sqrt{t_{1}^{2}+t_{2}^{2}+z_{i}^{2}}}}{\left(t_{1}^{2}+t_{2}^{2}+z_{i}^{2}\right)^{\frac{3}{2}}} \mathrm{~d} t_{2} \mathrm{~d} z_{i}
$$

Integrando respecto de $z_{i}$ :

$$
f\left(t_{1}\right)=\frac{2 K^{2}}{\pi} \int_{0}^{L_{2}}\left(L_{2}-t_{2}\right) \Gamma_{-1}\left(K \rho, K \sqrt{\rho^{2}+L_{3}^{2}}\right) \mathrm{d} t_{2}
$$

donde:

$$
\rho=\rho\left(t_{1}, t_{2}\right)=\sqrt{t_{1}^{2}+t_{2}^{2}}
$$

El DEA puede reescribirse:

$$
\begin{aligned}
\overline{g_{i} s_{j}} & =I_{1}\left(d-L_{1}^{(i)}, d, L_{1}^{(i)}-d\right)+ \\
& +I_{0}\left(d, d+L_{1}^{(j)}-L_{1}^{(i)}, L_{1}^{(i)}\right)+ \\
& +I_{-1}\left(d-L_{1}^{(j)}-L_{1}^{(i)}, d+L_{1}^{(j)}, d+L_{1}^{(j)}\right)
\end{aligned}
$$

donde:

$$
I_{s}(a, b, c)=\frac{2 K^{2}}{\pi} \int_{a}^{b} \int_{0}^{L_{2}}\left(c+s t_{1}\right)\left(L_{2}-t_{2}\right) g(\rho) \mathrm{d} t_{2} \mathrm{~d} t_{1}
$$

en la que:

$$
g(\rho)=\frac{2 K^{2}}{\pi} \Gamma_{-1}\left(K \rho, K \sqrt{\rho^{2}+L_{3}^{2}}\right)
$$

La expresión para $I_{s}(a, b, c)$ puede desarrollarse mediante el cambio se Ström:

$$
I_{s}(a, b, c)=\int_{a}^{b} \varphi_{1}(\rho) g(\rho) \rho \mathrm{d} \rho+\int_{b}^{\sqrt{a^{2}+L_{2}^{2}}} \varphi_{2}(\rho) g(\rho) \rho \mathrm{d} \rho+\int_{\sqrt{a^{2}+L_{2}^{2}}}^{\sqrt{b^{2}+L_{2}^{2}}} \varphi_{3}(\rho) g(\rho) \rho \mathrm{d} \rho
$$

siendo:

$$
\begin{aligned}
\varphi_{1}(\rho) & =c L_{2} \arccos \frac{a}{\rho}+\frac{s}{2}\left(a^{2}-\rho^{2}\right)+c(a-\rho)+s L_{2} \sqrt{\rho^{2}-a^{2}} \\
\varphi_{2}(\rho) & =c L_{2}\left(\arccos \frac{a}{\rho}-\arccos \frac{b}{\rho}\right)+\frac{s}{2}\left(a^{2}-b^{2}\right)+c(a-b)+ \\
& +s L_{2}\left(\sqrt{\rho^{2}-a^{2}}-\sqrt{\rho^{2}-b^{2}}\right) \\
\varphi_{3}(\rho) & =c L_{2}\left(\operatorname{arcsen} \frac{L_{2}}{\rho}-\arccos \frac{b}{\rho}\right)+\frac{s}{2}\left(\rho^{2}+a^{2}-b^{2}\right)-c b+ \\
& +c \sqrt{\rho^{2}-L_{2}^{2}}-s L_{2} \sqrt{\rho^{2}-b^{2}}
\end{aligned}
$$




\section{A.4.3.2. Intercambio entre un volumen de gas y la superficie de una pared final}

El intercambio entre un volumen de gas y la superficie de una pared final (\$3.2.4.2) viene dada por:

$$
\overline{g_{i} s_{j}}=\int_{0}^{L_{3}} \int_{0}^{L_{3}} \int_{0}^{L_{2}} \int_{0}^{L_{2}} \int_{d}^{d+L_{1}^{(i)}} \frac{K x_{i}}{\pi r^{3}} \mathrm{e}^{-K r} \mathrm{~d} x_{i} \mathrm{~d} y_{i} \mathrm{~d} y_{j} \mathrm{~d} z_{i} \mathrm{~d} z_{j}
$$

donde:

$$
r=\sqrt{x_{i}^{2}+\left(y_{j}-y_{i}\right)^{2}+\left(z_{j}-z_{i}\right)^{2}}
$$

Haciendo los cambios $t_{2}=y_{j}-y_{i}$ y $t_{3}=z_{j}-z_{i}$ :

$$
\overline{g_{i} s_{j}}=\frac{4 K}{\pi} \int_{0}^{L_{3}} \int_{0}^{L_{2}} \int_{d}^{d+L_{1}^{(i)}} x_{i}\left(L_{2}-t_{2}\right)\left(L_{3}-t_{3}\right) \frac{\mathrm{e}^{-K \sqrt{x_{i}^{2}+t_{2}^{2}+t_{3}^{2}}}}{\left(x_{i}^{2}+t_{2}^{2}+t_{3}^{2}\right)^{\frac{3}{2}}} \mathrm{~d} x_{i} \mathrm{~d} t_{2} \mathrm{~d} t_{3}
$$

Integrando respecto de $z_{i}$ :

$$
\overline{g_{i} s_{j}}=\int_{0}^{L_{2}} \int_{d}^{d+L_{1}^{(i)}} x_{i}\left(L_{2}-t_{2}\right) g(\rho) \mathrm{d} x_{i} \mathrm{~d} t_{2}
$$

siendo:

$$
\begin{aligned}
g(\rho) & =\frac{4 K}{\pi}\left(\frac{L_{3}^{2}}{\rho^{2}} \frac{\mathrm{e}^{-K \sqrt{\rho^{2}+L_{3}^{2}}}}{\sqrt{\rho^{2}+L_{3}^{2}}}-\frac{K L_{3}}{\rho} \mathrm{S}_{0}\left(K \rho, \operatorname{arctg} \frac{L_{3}}{\rho}\right)+\frac{L_{3}}{K \rho^{3}} \mathrm{~S}_{2}\left(K \rho, \operatorname{arctg} \frac{L_{3}}{\rho}\right)-\right. \\
& \left.-K \Gamma_{-1}\left(K \rho, K \sqrt{\rho^{2}+L_{3}^{2}}\right)\right)
\end{aligned}
$$

en la que:

$$
\rho=\rho\left(x_{i}, t_{2}\right)=\sqrt{x_{i}^{2}+t_{2}^{2}}
$$

La aplicación del cambio de Ström conduce finalmente a:

$$
\overline{g_{i} s_{j}}=\int_{d}^{d+L_{1}} \varphi_{1}(\rho) g(\rho) \rho \mathrm{d} \rho+\int_{d+L_{1}}^{\sqrt{d^{2}+L_{2}^{2}}} \varphi_{2}(\rho) g(\rho) \rho \mathrm{d} \rho+\int_{\sqrt{d^{2}+L_{2}^{2}}}^{\sqrt{\left(d+L_{1}\right)^{2}+L_{2}^{2}}} \varphi_{3}(\rho) g(\rho) \rho \mathrm{d} \rho
$$

siendo:

$$
\begin{aligned}
& \varphi_{1}(\rho)=\frac{1}{2}\left(d^{2}-\rho^{2}\right)+L_{2} \sqrt{\rho^{2}-d^{2}} \\
& \varphi_{2}(\rho)=\frac{1}{2}\left(d^{2}-\left(d+L_{1}\right)^{2}\right)+L_{2}\left(\sqrt{\rho^{2}-d^{2}}-\sqrt{\rho^{2}-\left(d+L_{1}\right)^{2}}\right) \\
& \varphi_{3}(\rho)=\frac{1}{2}\left(\rho^{2}+L_{2}^{2}-\left(d+L_{1}\right)^{2}\right)-L_{2} \sqrt{\rho^{2}-\left(d+L_{1}\right)^{2}}
\end{aligned}
$$


A.4.3.3. Intercambio entre un volumen de gas y la superficie de las baldosas en contacto con los rodillos

El intercambio directo entre un gas inferior y las baldosas (figura 3.17) puede calcularse mediante:

$$
\overline{g_{i} s_{j}}= \begin{cases}2\left(\frac{L_{1}}{\delta}-\left\lfloor\frac{x_{i}^{+}}{\delta}+\frac{1}{2}\right\rfloor\right) I_{\infty}+2 \sum_{k=1}^{\frac{x_{i}^{+}}{\delta}+\frac{1}{2}} I_{k} & \text { si están en el mismo sector } \\ \left\lfloor\frac{x_{i}^{+}}{\delta}+\frac{1}{2}\right\rfloor I_{\infty}-\sum_{k=1}^{\frac{x_{i}^{+}}{\delta}+\frac{1}{2}} I_{k} & \text { si están en sectores contiguos } \\ 0 & \text { en caso contrario }\end{cases}
$$

donde:

$$
\begin{aligned}
& I_{\infty}=\int_{0}^{d} \int_{0}^{L_{2}} \int_{0}^{L_{2}} \int_{0}^{x_{i}^{+}\left(z_{i}\right)} \int_{x_{j}^{-}\left(x_{i}, z_{i}\right)}^{x_{j}^{+}\left(x_{i}, z_{i}\right)} \frac{K\left(L_{3}-z_{i}\right)}{\pi r^{3}} \mathrm{e}^{-K r} \mathrm{~d} x_{j} \mathrm{~d} x_{i} \mathrm{~d} y_{i} \mathrm{~d} y_{j} \mathrm{~d} z_{i} \\
& I_{k}=\int_{0}^{d} \int_{0}^{L_{2}} \int_{0}^{L_{2}} \int_{0}^{\left(k+\frac{1}{2}\right) \delta} \int_{x_{j}^{-}\left(x_{i}, z_{i}\right)}^{x_{j}^{+}\left(x_{i}, z_{i}\right)} \frac{K\left(L_{3}-z_{i}\right)}{\pi r^{3}} \mathrm{e}^{-K r} \mathrm{~d} x_{j} \mathrm{~d} x_{i} \mathrm{~d} y_{i} \mathrm{~d} y_{j} \mathrm{~d} z_{i} \\
& r=\sqrt{\left(x_{j}-x_{i}\right)^{2}+\left(y_{j}-y_{i}\right)^{2}+\left(L_{3}-z_{i}\right)^{2}} \\
& x_{i}^{+}\left(z_{i}\right)=\left(L_{3}-R-z_{i}\right) \sqrt{\left(\frac{\delta}{2 R}\right)^{2}-1} \\
& x_{j}^{-}\left(x_{i}, z_{i}\right)=x_{i}-\left(L_{3}-R-z_{i}\right) \frac{x_{i}+\frac{\delta}{2}-R \sqrt{1-\frac{R^{2}-\left(x_{i}+\frac{\delta}{2}\right)^{2}}{\left(L_{3}-R-z_{i}\right)^{2}}}}{L_{3}-2 R-z_{i}} \\
& x_{j}^{+}\left(x_{i}, z_{i}\right)=x_{i}-\left(L_{3}-R-z_{i}\right) \frac{x_{i}-\frac{\delta}{2}+R \sqrt{1-\frac{R^{2}-\left(x_{i}-\frac{\delta}{2}\right)^{2}}{\left(L_{3}-R-z_{i}\right)^{2}}}}{L_{3}-2 R-z_{i}}
\end{aligned}
$$

Utilizando el cambio $t_{2}=y_{j}-y_{i}$ e integrando respecto de $t_{2}$ :

$$
\begin{aligned}
I_{\infty} & =\int_{0}^{d} \int_{0}^{L_{2}} \int_{0}^{x_{i}^{+}\left(z_{i}\right)} \int_{x_{j}^{-}\left(x_{i}, z_{i}\right)}^{x_{j}^{+}\left(x_{i}, z_{i}\right)}\left(L_{3}-z_{i}\right) g(\rho) \mathrm{d} x_{j} \mathrm{~d} x_{i} \mathrm{~d} t_{2} \mathrm{~d} z_{i} \\
I_{k} & =\int_{0}^{d} \int_{0}^{\left(k+\frac{1}{2}\right) \delta} \int_{x_{j}^{-}\left(x_{i}, z_{i}\right)}^{x_{j}^{+}\left(x_{i}, z_{i}\right)}\left(L_{3}-z_{i}\right) g(\rho) \mathrm{d} x_{j} \mathrm{~d} x_{i} \mathrm{~d} z_{i}
\end{aligned}
$$


en las que:

$$
\begin{aligned}
g(\rho) & =\frac{2 L_{2}}{\pi \rho}\left(K \frac{\mathrm{e}^{-K \sqrt{t_{2}^{2}+\rho^{2}}}}{\sqrt{t_{2}^{2}+\rho^{2}}}-K^{2} \mathrm{~S}_{0}\left(K \rho, \operatorname{arctg} \frac{L_{2}}{\rho}\right)+\frac{1}{\rho} \mathrm{S}_{2}\left(K \rho, \operatorname{arctg} \frac{L_{2}}{\rho}\right)\right)- \\
& -\frac{2 K^{2}}{\pi} \Gamma_{-1}\left(K \rho, K \sqrt{L_{2}^{2}+\rho^{2}}\right)
\end{aligned}
$$

donde:

$$
\rho=\rho\left(x_{i}, x_{j}, z_{i}\right)=\sqrt{\left(x_{j}-x_{i}\right)^{2}+\left(L_{3}-z_{i}\right)^{2}}
$$

Haciendo el cambio $t_{1}=x_{j}-x_{i}$ y definiendo:

$$
\begin{aligned}
t_{1}^{-}\left(x_{i}, z_{i}\right) & =-\left(L_{3}-R-z_{i}\right) \frac{x_{i}+\frac{\delta}{2}-R \sqrt{1-\frac{R^{2}-\left(x_{i}+\frac{\delta}{2}\right)^{2}}{\left(L_{3}-R-z_{i}\right)^{2}}}}{L_{3}-2 R-z_{i}} \\
t_{1}^{+}\left(x_{i}, z_{i}\right) & =-\left(L_{3}-R-z_{i}\right) \frac{x_{i}-\frac{\delta}{2}+R \sqrt{1-\frac{R^{2}-\left(x_{i}-\frac{\delta}{2}\right)^{2}}{\left(L_{3}-R-z_{i}\right)^{2}}}}{L_{3}-2 R-z_{i}}
\end{aligned}
$$

se tiene:

$$
\begin{aligned}
& I_{\infty}=\int_{0}^{L_{3}-R} \int_{t_{1}^{-}\left(x_{i}^{+}, z_{i}\right)}^{t_{1}^{+}\left(x_{i}^{+}, z_{i}\right)}\left(\left(L_{3}-z_{i}\right)\left(x_{i}^{+}+\frac{\delta}{2}\right)+\left(L_{3}-R-z_{i}\right) t_{1}-R \rho\right) g(\rho) \mathrm{d} t_{1} \mathrm{~d} z_{i}+ \\
& +\int_{0}^{L_{3}-R} \int_{t_{1}^{+}\left(x_{i}^{+}, z_{i}\right)}^{t_{1}^{-}}\left(\left(L_{3}-z_{i}\right) \delta-2 R \rho\right) g(\rho) \mathrm{d} t_{1} \mathrm{~d} z_{i}+ \\
& +\int_{0}^{L_{3}-R} \int_{t_{1}^{-}}^{t_{1}^{+}\left(0, z_{i}\right)}\left(\left(L_{3}-z_{i}\right) \frac{\delta}{2}-\left(L_{3}-R-z_{i}\right) t_{1}-R \rho\right) g(\rho) \mathrm{d} t_{1} \mathrm{~d} z_{i}
\end{aligned}
$$

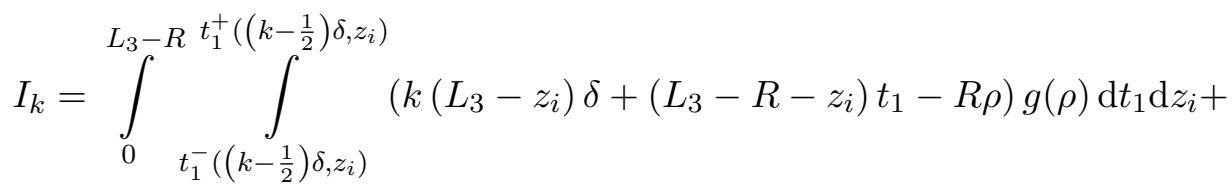

$$
\begin{aligned}
& +\int_{0}^{L_{3}-R} \int_{t_{1}^{+}\left(\left(k-\frac{1}{2}\right) \delta, z_{i}\right)}^{t_{1}^{-}\left(0, z_{i}\right)}\left(\left(L_{3}-z_{i}\right) \delta-2 R \rho\right) g(\rho) \mathrm{d} t_{1} \mathrm{~d} z_{i}+ \\
& +\int_{0}^{L_{3}-R} \int_{t_{1}^{-}\left(0, z_{i}\right)}^{t_{1}^{+}}\left(\left(L_{3}-z_{i}\right) \frac{\delta}{2}-\left(L_{3}-R-z_{i}\right) t_{1}-R \rho\right) g(\rho) \mathrm{d} t_{1} \mathrm{~d} z_{i}
\end{aligned}
$$




\section{A.5. DEA entre superficies en presencia de gases claros}

\section{A.5.1. Intercambio entre superficies planas paralelas}

Este DEA se obtuvo de la bibliografía [131,132]:

$$
\begin{aligned}
\overline{s_{i} s_{j}} & =\frac{L_{3}^{2}}{\pi}\left(\sqrt { A ^ { 2 } + 1 } \left(B \operatorname{arctg} \frac{B}{\sqrt{A^{2}+1}}-C \operatorname{arctg} \frac{C}{\sqrt{A^{2}+1}}-\right.\right. \\
& \left.-D \operatorname{arctg} \frac{D}{\sqrt{A^{2}+1}}+E \operatorname{arctg} \frac{E}{\sqrt{A^{2}+1}}\right)+ \\
& +A\left(\sqrt{B^{2}+1} \operatorname{arctg} \frac{A}{\sqrt{B^{2}+1}}-\sqrt{C^{2}+1} \operatorname{arctg} \frac{A}{\sqrt{C^{2}+1}}-\right. \\
& \left.-\sqrt{D^{2}+1} \operatorname{arctg} \frac{A}{\sqrt{D^{2}+1}}+\sqrt{E^{2}+1} \operatorname{arctg} \frac{A}{\sqrt{E^{2}+1}}\right)- \\
& -B \operatorname{arctg} B+C \operatorname{arctg} C+D \operatorname{arctg} D-E \operatorname{arctg} E+ \\
& \left.+\frac{1}{2} \ln \frac{\left(B^{2}+1\right)\left(A^{2}+C^{2}+1\right)\left(A^{2}+D^{2}+1\right)\left(E^{2}+1\right)}{\left(A^{2}+B^{2}+1\right)\left(C^{2}+1\right)\left(D^{2}+1\right)\left(A^{2}+E^{2}+1\right)}\right)
\end{aligned}
$$

en la que:

$$
\begin{aligned}
& A=\frac{L_{2}}{L_{3}} \\
& B=\frac{d-L_{1}^{(i)}}{L_{3}} \\
& C=\frac{d}{L_{3}} \\
& D=\frac{d+L_{1}^{(j)}-L_{1}^{(i)}}{L_{3}} \\
& E=\frac{d+L_{1}^{(j)}}{L_{3}}
\end{aligned}
$$

\section{A.5.2. Intercambio entre superficies planas perpendiculares}

Si el gas es claro, la expresión para $f\left(t_{1}\right)$ se simplifica a:

$$
f\left(t_{1}\right)=\int_{0}^{L_{3}} \int_{0}^{L_{2}} \frac{y_{i} z_{j}}{\pi r^{4}} \mathrm{~d} y_{i} \mathrm{~d} z_{j}=\frac{1}{4 \pi} \ln \frac{\left(t_{1}^{2}+L_{2}^{2}\right)\left(t_{1}^{2}+L_{3}^{2}\right)}{t_{1}^{2}\left(t_{1}^{2}+L_{2}^{2}+L_{3}^{2}\right)}
$$

\section{A.5.3. Intercambio entre una superficie de una pared final y una superficie plana}

Si el gas es claro, puede evaluarse $g(\rho)$ sin involucrar funciones especiales:

$$
g(\rho)=\lim _{K \rightarrow 0} \frac{2 K^{2}}{\pi} \Gamma_{-2}\left(K \rho, K \sqrt{\rho^{2}+L_{3}^{2}}\right)=\frac{1}{\pi} \frac{L_{3}^{2}}{\rho^{2}\left(\rho^{2}+L_{3}^{2}\right)}
$$




\section{A.5.4. Intercambio entre las superficies de las paredes finales}

El DEA entre las superficies de las paredes finales en presencia de un gas claro es bastante simple:

$$
\overline{s_{i} s_{j}}=\frac{4 d^{2}}{\pi} \int_{0}^{L_{3}} \int_{0}^{L_{2}} \frac{\left(L_{2}-t_{2}\right)\left(L_{3}-t_{3}\right)}{\left(d^{2}+t_{2}^{2}+t_{3}^{2}\right)^{2}} \mathrm{~d} t_{2} \mathrm{~d} t_{3}
$$

en versión integrada:

$$
\begin{aligned}
\overline{s_{i} s_{j}} & =\frac{2 d^{2}}{\pi}\left(A \sqrt{B^{2}+1} \operatorname{arctg} \frac{A}{\sqrt{B^{2}+1}}+B \sqrt{A^{2}+1} \operatorname{arctg} \frac{B}{\sqrt{A^{2}+1}}-\right. \\
& \left.-A \operatorname{arctg} A-B \operatorname{arctg} B+\frac{1}{2} \ln \frac{\left(A^{2}+1\right)\left(B^{2}+1\right)}{A^{2}+B^{2}+1}\right)
\end{aligned}
$$

siendo:

$$
\begin{aligned}
& A=\frac{L_{2}}{d} \\
& B=\frac{L_{3}}{d}
\end{aligned}
$$

A.5.5. Intercambio entre la superficie de la solera y la superficie de las baldosas en contacto con los rodillos

Si el gas es claro:

$$
g(\rho)=\frac{2 L_{3}}{\pi} \int_{0}^{L_{2}} \frac{L_{2}-t_{2}}{\left(t_{2}^{2}+\rho^{2}\right)^{2}} \mathrm{~d} t_{2}=\frac{L_{2} L_{3}}{\pi \rho^{3}} \operatorname{arctg} \frac{L_{2}}{\rho}
$$

A.5.6. Intercambio entre la superficie de las paredes laterales y la superficie de las baldosas en contacto con los rodillos

En el límite en el que $K \rightarrow 0$ :

$$
g(\rho)=\lim _{K \rightarrow 0} \frac{K^{2}}{\pi} \Gamma_{-2}\left(K \rho, K \sqrt{L_{2}+\rho^{2}}\right)=\frac{1}{2 \pi} \frac{L_{2}^{2}}{\rho^{2}\left(L_{2}^{2}+\rho^{2}\right)}
$$

A.5.7. Intercambio entre la superficie de una pared final y la superficie de las baldosas en contacto con los rodillos

Cuando el gas es claro:

$$
\begin{aligned}
g\left(\rho, z_{i}\right) & =\lim _{K \rightarrow 0} \frac{2 K^{2}}{\pi} \Gamma_{-2}\left(K \sqrt{t_{1}^{+}\left(z_{i}\right)^{2}+\rho^{2}}, K \sqrt{t_{1}^{-}\left(z_{i}\right)^{2}+\rho^{2}}\right)= \\
& =\frac{1}{\pi} \frac{t_{1}^{-}\left(z_{i}\right)^{2}-t_{1}^{+}\left(z_{i}\right)^{2}}{\left(t_{1}^{+}\left(z_{i}\right)^{2}+\rho^{2}\right)\left(t_{1}^{-}\left(z_{i}\right)^{2}+\rho^{2}\right)}
\end{aligned}
$$




\section{A.5.8. Intercambio entre la superficie de las baldosas en contacto con los rodillos y la superficie de los rodillos}

Este DEA puede determinarse a partir de la ley de las cuerdas cruzadas de Hottel [133]:

$$
\overline{s_{i} s_{j}}=L_{1} L_{2}\left(1+Y \arccos Y-\sqrt{1-Y^{2}}\right)
$$

siendo:

$$
Y=\frac{2 R}{\delta}
$$

\section{A.5.9. Intercambio entre las superficies de los rodillos}

El DEA entre los rodillos cuando $K=0$ puede obtenerse también mediante la ley de las cuerdas cruzadas (ejemplo 7-16 de la referencia [45]):

$$
\overline{s_{i} s_{j}}=\frac{4 L_{1} L_{2} R}{\delta}\left(\operatorname{arcsen} Y+\frac{1}{Y}\left(\sqrt{1-Y^{2}}-1\right)\right)
$$

donde:

$$
Y=\frac{2 R}{\delta}
$$

\section{A.6. DEA en presencia de gases opacos}

Si el coeficiente de absorción del gas gris es lo suficientemente elevado, se puede considerar que es opaco a la radiación. Si se da esta circunstancia, se cumple:

$$
\overline{s_{i} s_{j}}=0
$$

ya que el gas absorbe toda la radiación emitida por las superficies. Por lo tanto, si el volumen $V_{i}$ se encuentra en contacto con la superficie $A_{j}$ :

$$
\overline{g_{i} s_{j}}=A_{j}
$$

En caso contrario, $\overline{g_{i} s_{j}}=0$.

Del mismo modo, las DEA entre volúmenes de gas contiguos serán:

$$
\overline{g_{i} g_{j}}=A_{k}
$$

donde $A_{k}$ es el área de la superficie ficticia que delimita ambos volúmenes de gas. El DEA entre un volumen de gas y él mismo puede deducirse a partir de la segunda relación de conservación (1.32):

$$
\overline{g_{i} g_{i}}=4 K V_{i}-\sum_{k} A_{k}
$$

donde $A_{k}$ son las áreas de las superficies (reales o ficticias) que envuelven al volumen $V_{i}$. Las DEA entre cualquier otra pareja de gases obedecerán $\overline{g_{i} g_{j}}=0$. 


\section{A.7. Coeficiente de absorción medio}

En el artículo original de Hottel y Cohen [41] se presenta un tratamiento aplicable para el caso en el que el coeficiente de absorción del gas no es uniforme en el cerramiento, del que se han propuesto variantes más desarrolladas [134] y más simplificadas [91].

La composición de los humos en los distintos sectores en los que se divida el horno de rodillos (§3.1.2) será, en general, diferente. En consecuencia, debe contemplarse la posibilidad de que se den modificaciones en el coeficiente de absorción del gas a lo largo del horno, para lo que se recurrió al planteamiento de Hottel y Cohen.

Teniendo en cuenta que el coeficiente de absorción puede ser distinto en cada sector, pueden redefinirse las DEA de la manera siguiente:

$$
\begin{aligned}
\overline{s_{i} s_{j}} & =\iint_{A_{j}} \iint_{A_{i}} \frac{\cos \theta_{i} \cos \theta_{j}}{\pi r^{2}} \mathrm{e}^{-K r} \mathrm{~d} A_{i} \mathrm{~d} A_{j} \\
\overline{s_{i} g_{j}} & =\iiint_{V_{j}} \iint_{A_{i}} \frac{\cos \theta_{i} K^{(j)}}{\pi r^{2}} \mathrm{e}^{-K r} \mathrm{~d} A_{i} \mathrm{~d} V_{j} \\
\overline{g_{i} g_{j}} & =\iiint_{V_{j}} \iiint_{V_{i}} \frac{K^{(i)} K^{(j)}}{\pi r^{2}} \mathrm{e}^{-K r} \mathrm{~d} V_{i} \mathrm{~d} V_{j}
\end{aligned}
$$

en las que $K^{(i)}$ y $K^{(j)}$ denotan a los coeficientes de absorción de los gases contenidos en los sectores $(i)$ y $(j)$, respectivamente, y $K$ hace referencia a un coeficiente de absorción medio en el camino que separa los sectores $(i)$ y $(j)$.

El coeficiente de absorción medio se ha calculado mediante las siguientes expresiones:

- Intercambio entre zonas pertenecientes, respectivamente, a los sectores $(i)$ y $(j)$ :

$$
K=\frac{\frac{K^{(i)} L_{1}^{(i)}+K^{(j)} L_{1}^{(j)}}{2}+\sum_{k=i+1}^{j-1} K^{(k)} L_{1}^{(k)}}{\frac{L_{1}^{(i)}+L_{1}^{(j)}}{2}+\sum_{k=i+1}^{j-1} L_{1}^{(k)}}
$$

- Intercambio entre la superficie de una pared final y una zona en el sector $(j)$ :

$$
K=\frac{\frac{K^{(j)} L_{1}^{(j)}}{2}+\sum_{k=1}^{j-1} K^{(k)} L_{1}^{(k)}}{\frac{L_{1}^{(j)}}{2}+\sum_{k=1}^{j-1} L_{1}^{(k)}}
$$

- Intercambio entre las superficies de las paredes finales:

$$
K=\frac{\sum_{k=1}^{n} K^{(k)} L_{1}^{(k)}}{\sum_{k=1}^{n} L_{1}^{(k)}}
$$





\section{Bibliografía}

[1] Anónimo, Scala graduum caloris: calorum descriptiones et signa. Philos. T. Roy. Soc., vol. 22, pp. 824-829, 1701.

[2] UNE-EN 14411:2007, Baldosas cerámicas. Definiciones, clasificación, características y marcado.

[3] ISO 13006:2010, Baldosas cerámicas. Definiciones, clasificación, características y marcado.

[4] M. González, A. Miralles, R. Monfort, L. Montón, J. R. Castaño, A. Pitarch, F. C. de Mazarredo, J. J. Palencia, A. García, J. E. Enrique, G. Silva, A. Muñoz, J. Mira, T. Ros, R. Benedé, M. García, L. E. Domínguez, F. Pla, V. Cerdán y C. Jareño, Guía de la baldosa cerámica, $6 .^{a}$ ed. Valencia: Instituto Valenciado de la Edificación, 2011.

[5] E. Sánchez, J. García-Ten, P. Quereda y V. Beltrán, Estudio de composiciones de gres rojo de muy baja porosidad. Técnica Cerámica, vol. 305, pp. 902-909, 2002.

[6] A. Blasco, A. Escardino, G. Busani, E. Monfort, J. L. Amorós, J. E. EnRique, V. Beltrán y P. Negre, Tratamiento de emisiones gaseosas, efluentes líquidos y residuos sólidos de la industria cerámica. Castellón: Instituto de Tecnología Cerámica, 1992.

[7] E. Monfort, A. Mezquita, R. Granel, E. Vaquer, A. Escrig, A. Miralles y V. Zaera, Análisis de consumos energéticos y emisiones de dióxido de carbono en la fabricación de baldosas cerámicas. Bol. Soc. Esp. Ceram. Vidr., vol. 49, pp. 303-310, 2010.

[8] E. Monfort, A. Mezquita, G. Mallol, R. Granel y E. Vaquer, Informe sectorial de consumos energéticos y emisiones de dióxido de carbono en el proceso de fabricación de baldosas cerámicas. Valencia: Agencia Valenciana de la Energía, 2011.

[9] G. Nassetti, F. Ferrari, A. Fregni y G. Maestri, Piastrelle ceramiche 8 energia: banca dati dei consumi energetici nell'industria della piastrelle di ceramica. Bolonia: Centro Ceramico, 1998.

[10] M. Monzó, J. E. Enrique, J. De la Torre y J. L. Amorós, Defectos de los productos de monococción (y V). Defectos producidos en la cocción. Técnica Cerámica, vol. 170, pp. 18-27, 1989.

[11] E. Monfort, A. Mezquita, E. Vaquer, G. Mallol y D. Gabaldón-Estevan, La evolución energética del sector español de baldosas cerámicas. Bol. Soc. Esp. Ceram. Vidr., vol. 53, pp. 111-120, 2014. 
[12] J. L. Amorós, J. E. Enrique, A. Blasco y R. Benavent, Hornos para la fabricación de pavimentos y revestimientos cerámicos. Características y criterios de elección. Técnica Cerámica, vol. 104, pp. 750-768, 1982.

[13] J. E. Enrique, J. L. Amorós y M. Monzó, Tecnología cerámica, vol. IV. Secado y cocción. Valencia: Instituto de Química Técnica. Universidad de Valencia, 1985.

[14] SACMi y Asociación Española de Técnicos Cerámicos, Tecnología cerámica aplicada, vol. II. Castellón: Faenza Editrice Ibérica, 2004.

[15] L. Lorici, El horno de rodillos y su influencia sobre la planaridad del producto final. Cerámica y Cristal, vol. 114, pp. 19-28, 1995.

[16] A. Mezquita, J. Boix, E. Monfort y G. Mallol, Energy saving in ceramic tile kilns: cooling gas heat recovery. Appl. Therm. Eng., vol. 65, pp. 102-110, 2014.

[17] C. Ferrer, D. Llorens, G. Mallol, E. Monfort y A. Moreno, Optimización de las condiciones de funcionamiento en hornos monoestrato (III). Medida de gradientes transversales de temperatura. Técnica Cerámica, vol. 227, pp. 653-662, 1994.

[18] A. Blasco, L. Carda, G. Mallol y E. Monfort, Optimización de las condiciones de funcionamiento en hornos monoestrato (I). Curva de presiones. Técnica Cerámica, vol. 206, pp. 585-592, 1992.

[19] A. Blasco, J. E. Enrique, G. Mallol y E. Monfort, Optimización de las condiciones de funcionamiento en hornos monoestrato (II). Caudal de aire de combustión. Técnica Cerámica, vol. 218, pp. 716-729, 1993.

[20] P. Lybaert, Simulation numérique des fours de cuisson céramique. En: Conduite optimisée des fours dans l'industrie céramique. Seminaire COMETT, 1995.

[21] S. Ferrer, A. Mezquita, M. P. Gómez-Tena, C. Machí y E. Monfort, Estimation of the heat of reaction in traditional ceramic compositions. Appl. Clay Sci., vol. 108, pp. 28-39, 2015.

[22] A. Mezquita, E. Monfort, E. Vaquer, S. Ferrer, M. A. Arnal, J. Toledo y M. A. Cuesta, Optimización energética en la fabricación de baldosas cerámicas mediante el uso de aceite térmico. Bol. Soc. Esp. Ceram. Vidr., vol. 51, pp. 183-190, 2012.

[23] S. Ferrer, Análisis energético y exergético del proceso de cocción de baldosas cerámicas. Tesis Doctoral. Castellón: Universitat Jaume I, 2016.

[24] A. Blasco, J. L. Amorós, V. Beltrán y F. Negre, Mejora de producción y calidad de producto en la fabricación de pavimentos de gres, acoplando un secadero túnel de vagonetas a la entrada del horno. Diseño del secadero. Técnica Cerámica, vol. 130, pp. 18-29, 1985.

[25] E. Monfort, A. Mezquita, G. Mallol, R. Granel y E. Vaquer, Guía de ahorro energético en el sector de baldosas cerámicas. Valencia: Agencia Valenciana de la Energía, 2011.

[26] J. C. Jarque, A. Moreno, J. E. Enrique y A. Barba, Optimización de las condiciones de funcionamiento en hornos monoestrato (IV). Mecanismos de transmisión de energía calorífica. Técnica Cerámica, vol. 247, pp. 566-572, 1996. 
[27] V. Cantavella, Simulación de la deformación de baldosas cerámicas durante la cocción. Tesis Doctoral. Castellón: Universitat Jaume I, 1998.

[28] J. E. Enrique, V. Cantavella, A. Moreno y F. Negre, Estimating inner temperature profile in firing tile. Am. Ceram. Soc. Bull., vol. 77, pp. 77-80, 1998.

[29] V. Cantavella, A. Moreno, A. Mezquita, D. Llorens, J. Barberá y A. PaLANQUES, Distribución de temperaturas en el interior de una pieza durante la cocción industrial. En: Qualicer 2006: IX Congreso mundial de la calidad del azulejo y del pavimento cerámico, pp. P.BC151-P.BC164, 2006.

[30] H. S. Carslaw y J. C. Jaeger, Conduction of heat in solids, 2. ${ }^{\mathrm{a}}$ ed. Londres: Oxford University Press, 1959.

[31] A. Bejan, Theory of rolling contact heat transfer. J. Heat Transfer, vol. 111, pp. 257-263, 1989.

[32] H. HeRTz, Über die Berührung fester elastischer Körper. J. Reine Angew. Math., vol. 92, pp. 156-171, 1881.

[33] E. Specht y K. U. Holzapfel, Compensation of the heat transfer on the upperside and under-side in roller-kilns. En: Proceedings of the 5th European conference on industrial furnaces and boilers, pp. 577-586, 2000.

[34] M. Chmielowski y E. Specht, Modelling of the heat transfer of transport rollers in kilns. Appl. Therm. Eng., vol. 26, pp. 736-744, 2006.

[35] K. U. Holzapfel y E. Specht, Heat transfer between a rotating cylinder and a transported plate. Exp. Heat Transfer, vol. 19, pp. 39-51, 2006.

[36] J. Alves e Sousa, J. Ward y R. A. Wallis, An experimental study of convection in an industrial gas-fired heat treatment furnace. En: Proceedings of the ASME heat transfer division, vol. 317-2, pp. 541-548, 1995.

[37] R. Viskanta y M. P. MengüÇ, Radiation heat transfer in combustion systems. Prog. Energy Combust. Sci., vol. 13, pp. 97-160, 1987.

[38] R. G. Siddall, Flux methods for the analysis of radiant heat transfer. J. Inst. Fuel, vol. 47, pp. 101-109, 1974.

[39] J. R. Howell, The Monte Carlo method in radiative heat transfer. J. Heat Transfer, vol. 120 , pp. 547-570, 1998.

[40] H. A. J. Vercammen y G. F. Froment, An improved zone method using Monte Carlo techniques for the simulation of radiation in industrial furnaces. Int. J. Heat Mass Transfer, vol. 23, no. 3, pp. 329-337, 1980.

[41] H. C. Hottel y E. S. Cohen, Radiant heat exchange in a gas-filled enclosure: allowance for nonuniformity of gas temperature. A. I. Ch. E. Journal, vol. 4, pp. 3-14, 1958.

[42] E. S. CoHen, Effect of gas temperature gradients on radiant heat transmission. Tesis Doctoral. Cambridge: Massachusetts Institute of Technology, 1955.

[43] A. F. SARofim, Radiant heat transmission in enclosures. Tesis Doctoral. Cambridge: Massachusetts Institute of Technology, 1962. 
[44] H. C. Hottel y A. F. Sarofim, Radiative transfer. Nueva York: McGraw-Hill, 1967.

[45] R. Siegel y J. R. Howell, Thermal radiation heat transfer, 2. ${ }^{\text {a }}$ ed. Nueva York: McGraw-Hill, 1981.

[46] J. J. Noble, The zone method: explicit matrix relations for total exchange areas. Int. J. Heat Mass Transfer, vol. 18, pp. 261-269, 1975.

[47] G. El Hitti, M. Nemer y K. El Khoury, Reducing CPU time for radiative exchange and transient heat transfer analysis using zone method. Numer. Heat Transfer, vol. B56, pp. 23-37, 2009.

[48] G. El Hitti, M. Nemer, K. El Khoury y D. Clodic, The re-plating algorithm for radiation total exchange area calculation. Numer. Heat Transfer, vol. B57, pp. $110-125,2010$.

[49] J. S. Truelove, A mixed grey gas model for flame radiation. United Kingdom Atomic Energy Authority, Informe técnico AERE-R-8494, 1976.

[50] N. Lallemant, A. Sayret y R. Weber, Evaluation of emissivity correlations for $\mathrm{H}_{2} \mathrm{O}-\mathrm{CO}_{2}-\mathrm{N}_{2}$ /air mixtures and coupling with solution methods of the radiative transfer equation. Prog. Energy Combust. Sci., vol. 22, pp. 543-574, 1996.

[51] A. Marcu, W. Stoefs, K. Tuokko, C. Egenhofer, A. Renda, F. Simonelli, F. Genoese, E. Storti, E. Drabik, T. Hähl, M. Overgaag, K. Grave, M. Koper, G. Luchetta, M. Freudenthaler y A. Bolognini, Composition and drivers of energy prices and costs: case studies in selected energy-intensive industries. Comisión Europea, Informe técnico 409/PP/2014/FC Lot 1, 2016.

[52] A. Escardino, El esfuerzo en innovación de la industria cerámica de la Comunidad Valenciana para reducir las emisiones de dióxido de carbono. En: Simposio internacional sobre el cambio climático, desde la ciencia a la sociedad, pp. 121-133, 2005.

[53] C. Agrafiotis y T. Tsoutsos, Energy saving technologies in the European ceramic sector: a systematic review. Appl. Therm. Eng., vol. 21, pp. 1231-1249, 2001.

[54] D. Gabaldón-Estevan, A. Mezquita, S. Ferrer y E. Monfort, Unwanted effects of European Union environmental policy to promote a post-carbon industry. The case of energy in the European ceramic tile sector. J. Clean. Prod., vol. 117, pp. 41-49, 2016.

[55] A. Mezquita, E. Monfort y V. Zaera, Sector azulejero y comercio de emisiones: reducción de emisiones de $\mathrm{CO}_{2}$, benchmarking europeo. Bol. Soc. Esp. Ceram. Vidr., vol. 48, pp. 211-222, 2009.

[56] A. A. Boateng y P. V. BARR, A thermal model for the rotary kiln including heat transfer within the bed. Int. J. Heat Mass Transfer, vol. 39, pp. 2131-2147, 1996.

[57] M. A. Martins, L. S. Oliveira y A. S. Franca, Modeling and simulation of petroleum coke calcination in rotary kilns. Fuel, vol. 80, pp. 1611-1622, 2001.

[58] F. Marias, H. Roustan y A. Pichat, Modelling of a rotary kiln for the pyrolysis of aluminium waste. Chem. Eng. Sci., vol. 60, pp. 4609-4622, 2005. 
[59] L. Post, Modelling of flow and combustion in a glass melting furnace. Tesis Doctoral. Delft: Technische Universiteit Delft, 1988.

[60] R. Viskanta, Review of three-dimensional mathematical modeling of glass melting. J. Non-Cryst. Solids, vol. 177, pp. 347-362, 1994.

[61] M. V. Ramana Rao, P. M. Plehiers y G. F. Froment, The coupled simulation of heat transfer and reaction in a pyrolysis furnace. Chem. Eng. Sci., vol. 43, pp. 1223-1229, 1988.

[62] P. M. Plehiers, G. C. Reyniers y G. F. Froment, Simulation of the run length of an ethane cracking furnace. Ind. Eng. Chem. Res., vol. 29, pp. 636-641, 1990.

[63] G. J. Heynderickx, A. J. Oprins, G. B. Marin y E. Dick, Three-dimensional flow patterns in cracking furnaces with long-flame burners. A. I. Ch. E. Journal, vol. 47, pp. 388-400, 2001.

[64] G. Hu, H. Wang, F. Qian, K. M. Van Geem, C. M. Schietekat y G. B. MARIN, Coupled simulation of an industrial naphtha cracking furnace equipped with long-flame and radiation burners. Comput. Chem. Eng., vol. 38, pp. 24-34, 2012.

[65] Z. Li, P. V. Barr y J. K. Brimacombe, Computer simulation of the slab reheating furnace. Can. Metall. Quart., vol. 27, pp. 187-196, 1988.

[66] K. S. Chapman, S. Ramadhyani y R. Viskanta, Modeling and parametric studies of heat transfer in a direct-fired batch reheating furnace. J. Heat Treating, vol. 8, pp. 137-146, 1990.

[67] K. S. Chapman, S. Ramadhyani y R. Viskanta, Modeling and parametric studies of heat transfer in a direct-fired continuous reheating furnace. Metall. Trans. B, vol. 22, pp. 513-521, 1991.

[68] A. Emadi, A. Saboonchi, M. Taheri y S. Hassanpour, Heating characteristics of billet in a walking hearth type reheating furnace. Appl. Therm. Eng., vol. 63, pp. 396-405, 2014.

[69] Y. Hu, C. K. Tan, J. Broughton y P. A. Roach, Development of a first-principles hybrid model for large-scale reheating furnaces. Appl. Energ., vol. 173, pp. 555-566, 2016 .

[70] R. Méchi, H. Farhat, K. Halouani y M.-S. Radhouani, Modélisation des transferts radiatifs dans un incinérateur des émissions polluantes de la pyrolyse du bois. Int. J. Therm. Sci., vol. 43, pp. 697-708, 2004.

[71] D. R. Dugwell y D. E. OAKLey, A model of heat transfer in tunnel kilns used for firing refractories. Int. J. Heat Mass Transfer, vol. 31, pp. 2381-2390, 1988.

[72] P. Lybaert, M. El Hayek y H. Meunier, Numerical modelling of ceramics firing kilns. Application to the improvement of energy efficiency and product quality. En: Energy efficiency in process technology, P. Pilavachi (Ed.). Londres: Springer, 1993.

[73] S. Kaya, K. KÜÇÜKada y E. MançUhan, Model-based optimization of heat recovery in the cooling zone of a tunnel kiln. Appl. Therm. Eng., vol. 28, pp. 633-641, 2008. 
[74] J. UChe y J. M. Marín, Simulación y optimización de un horno cerámico monocapa. Bol. Soc. Esp. Ceram. Vidr., vol. 40, pp. 377-383, 2001.

[75] J. Alves e Sousa, S. C. Correia, J. Ward y M. Nogueira, A transient, multidimensional, zone model of a roller kiln used for firing ceramic tiles. En: Proceedings of the 6th European conference on industrial furnaces and boilers, vol. IV, pp. 169$182,2002$.

[76] J. M. Rhine y R. J. Tucker, Modelling of gas-fired furnaces and boilers. Nueva York: McGraw-Hill, 1991.

[77] M. Dal Bó, V. Cantavella, E. Sánchez, D. Hotza y A. Boschi, Modelización mecánica del enfriamiento rápido en sistemas tipo gres porcelánico. Bol. Soc. Esp. Ceram. Vidr., vol. 51, pp. 95-102, 2012.

[78] D. E. Knuth, Computer programming as an art. Commun. ACM, vol. 17, pp. 667-673, 1974.

[79] J. G. Knudsen, J. B. Kenneth, A. D. Holt, H. C. Hottel, A. F. Sarofim, F. C. Standiford, D. Stuhlbarg y V. W. Uhl, Heat transmission. En: Perry's

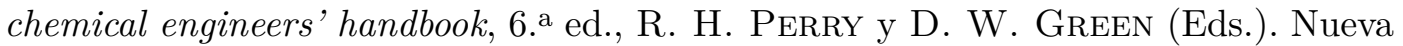
York: McGraw-Hill, 1984.

[80] D. Lide (Ed.), CRC handbook of chemistry and physics, $74 .^{\text {a }}$ ed. Boca Ratón: CRC Press, 1993.

[81] P. J. Coelho, J. M. Gonçalves, M. G. Carvalho y D. N. Trivic, Modelling of radiative heat transfer in enclosures with obstacles. Int. J. Heat Mass Transfer, vol. 41, pp. 745-756, 1998.

[82] B. J. Leon, Lumped systems. Nueva York: Holt, Rinehart and Winston, 1972.

[83] H. C. Hottel y A. F. SArofim, Models of radiative transfer in furnaces. J. Eng. Phys., vol. 19, pp. 1102-1114, 1970.

[84] M. R. Palmer, Modelling high temperature plant. British Gas Midlands Research Station, Informe técnico E521, 1988.

[85] H. ERKKu, Radiant heat exchange in gas-filled slabs and cylinders. Tesis Doctoral. Cambridge: Massachusetts Institute of Technology, 1959.

[86] H. C. Hottel y A. F. SARofim, The effect of gas flow patterns on radiative transfer in cylindrical furnaces. Int. J. Heat Mass Transfer, vol. 8, pp. 1153-1169, 1965.

[87] T. K. Kim y T. F. Smith, Radiative and conductive transfer for a real gas in a cylindrical enclosure with gray walls. Int. J. Heat Mass Transfer, vol. 28, pp. 2269$2277,1985$.

[88] E. R. Cohen, T. Cvitaš, J. G. Frey, B. Holmström, K. Kuchitzu, R. Marquardt, I. Mills, F. Pavese, M. Quack, J. Stohner, H. L. Strauss, M. TaKAMI y A. J. ThOR, Quantities, units and symbols in physical chemistry, 3. ${ }^{\mathrm{a}}$ ed. Cambridge: IUPAC \& RSC Publishing, 2008.

[89] H. B. BeckeR, A mathematical solution for gas-to-surface radiative exchange area for a rectangular parallelepiped enclosure containing a gray medium. J. Heat Transfer, vol. 99, pp. 203-207, 1977. 
[90] M. E. Larsen y J. R. Howell, The exchange factor method: an alternative basis for zonal analysis of radiating enclosures. J. Heat Transfer, vol. 107, pp. 936-942, 1985 .

[91] T. R. Johnson y J. M. Beer, Radiative heat transfer in furnaces: further development of the zone method of analysis. En: Proceedings of the 14th international symposium on combustion, pp. 639-649, 1973.

[92] M. Abramowitz y I. A. Stegun, Handbook of mathematical functions with formulas, graphs and mathematical tables. Nueva York: Dover, 1972.

[93] D. A. Lawson y C. D. Ziesler, An accurate program for radiation modelling in the design of high-temperature furnaces. IMA J. Math. Appl. Bus. Indust., vol. 7, pp. 109-116, 1996.

[94] W. Zhou y T. QIU, Zone modeling of radiative heat transfer in industrial furnaces using adjusted Monte-Carlo integral method for direct exchange area calculation. Appl. Therm. Eng., vol. 81, pp. 161-167, 2015.

[95] R. J. Tucker, Direct exchange areas for calculating radiation transfer in rectangular furnaces. J. Heat Transfer, vol. 108, pp. 707-710, 1986.

[96] H. Ebrahimi, A. Zamaniyan, J. S. S. Mohammadzadeh y A. A. Khalili, Zonal modeling of radiative heat transfer in industrial furnaces using simplified model for exchange area calculation. Appl. Math. Model., vol. 37, pp. 8004-8015, 2013.

[97] M. E. LARsen y J. R. Howell, Least-squares smoothing of direct exchange areas in zonal analysis. J. Heat Transfer, vol. 108, pp. 239-242, 1986.

[98] J. V. Leersum, A method for determining a consistent set of radiation view factor from a set generated by a nonexact method. Int. J. Heat Fluid Flow, vol. 10, pp. 83-85, 1989.

[99] C. V. S. Murty y B. S. N. Murty, Significance of exchange area adjustment in zone modelling. Int. J. Heat Mass Transfer, vol. 34, pp. 499-503, 1991.

[100] D. A. Lawson, An improved method for smoothing approximate exchange areas. Int. J. Heat Mass Transfer, vol. 38, pp. 3109-3110, 1995.

[101] K. J. Daun, D. P. Morton y J. R. Howell, Smoothing Monte Carlo exchange factors through constrained maximum likelihood estimation. ASME J. Heat Transfer, vol. 127, pp. 1124-1128, 2005.

[102] M. MÁrquez, Combustión y quemadores. Barcelona: Marcombo, 2005.

[103] National Institute of Standards and Technology, NIST chemistry webbook. [En línea], consulta: 2010-07-29. <http://webbook.nist.gov/chemistry/>

[104] G. Elmi, Le four à rouleaux. En: Conduite optimisée des fours dans l'industrie céramique. Seminaire COMETT, 1995.

[105] J. E. Enrique, A. Blasco, M. Fuster y J. L. Amorós, Tablas cerámicas, 2. a ed. Valencia: Instituto de Química Técnica. Universidad de Valencia, 1984.

[106] S. W. Churchill y H. H. S. Chu, Correlating equations for laminar and turbulent free convection from a vertical plate. Int. J. Heat Mass Transfer, vol. 18, pp. 13231329, 1975. 
[107] M. Al-Arabi y M. K. El-Riedy, Natural convection heat transfer from isothermal horizontal plates of different shapes. Int. J. Heat Mass Transfer, vol. 19, pp. 13991404, 1976.

[108] M. Fishenden y O. A. Saunders, An Introduction to heat transfer. Oxford: Clarendon Press, 1950.

[109] J.-M. Briot, B. Bourouga y J.-P. Bardon, Etude de la conductance thermique de transport entre les bagues d'un roulement à rouleaux. Rev. Gén. Therm., vol. 36, pp. 610-623, 1997.

[110] M. H. N. Naraghi y B. T. F. Chung, A unified matrix formulation for the zone method: a stochastic approach. Int. J. Heat Mass Transfer, vol. 28, pp. 245-251, 1985.

[111] Z.-F. Shen, T. F. Smith y P. Hix, Linearization of the radiation terms for improved convergence by use of the zone method. Numer. Heat Transfer, vol. A6, pp. 377-382, 1983.

[112] G. H. Golub y C. F. VAn Loan, Matrix computations, $4 .^{\text {a }}$ ed. Baltimore: Johns Hopkins University Press, 2013.

[113] B. Stroustrup, The $C++$ programming language, $4 .^{\mathrm{a}}$ ed. Boston: Addison-Wesley, 2013.

[114] ISO/IEC 14882:2014, Programming languages-C++.

[115] T. L. Anderson, Fracture mechanics: fundamentals and applications, $3 .^{\mathrm{a}}$ ed. Boca Ratón: CRC Press, 2005.

[116] A. Barba, V. Beltrán, C. Feliu, J. García-Ten, F. Ginés, E. Sánchez y V. SANZ, Materias primas para la fabricación de soportes de baldosas cerámicas, 2. ${ }^{a}$ ed. Castellón: Instituto de Tecnología Cerámica, 2002.

[117] EnagÁs, Calidad del gas por municipio. [En línea], consulta: 2012-05-21. <http: //www.enagas.es/>

[118] EnaGás, Calidad del gas por municipio. [En línea], consulta: 2015-04-17. <http: //www.enagas.es/>

[119] Enagás, Calidad del gas por municipio. [En línea], consulta: 2016-06-02. <http: //www.enagas.es/>

[120] M. Azara, J. E. Enrique, A. Blasco y J. L. Amorós, Ahorro energético en el sector azulejero, 2. ${ }^{\mathrm{a}}$ ed. Valencia: Conselleria d'Indústria, Comerç i Turisme, 1990.

[121] J. L. Amorós, V. Beltrán, A. Blasco, J. E. Enrique, A. Escardino y F. NeGRE, Defectos de fabricación de pavimentos y revestimientos cerámicos. Castellón: Instituto de Tecnología Cerámica, 1991.

[122] F. Sandoval y A. Ibáñez, Fast-fired wollastonite-based wall tile. Am. Ceram. Soc. Bull., vol. 84, pp. 9301-9303, 2005.

[123] V. Bargues, F. Calomarde, A. Orenga, J. García-Ten, P. Quereda y A. Mezquita, Utilización de la wollastonita en la fabricación de azulejos. En: Qualicer 2010: XI Congreso mundial de la calidad del azulejo y del pavimento cerámico, 2010. 
[124] J. E. Enrique, V. Cantavella, F. Negre y E. Sánchez, Simulazione della deformazione delle piastrelle ceramiche durante la cottura. Cer. Acta, vol. 11, pp. 31-41, 1999.

[125] J. E. Enrique, V. Cantavella, F. Negre y E. Sánchez, Model predicts tile deformation in firing. Am. Ceram. Soc. Bull., vol. 68, pp. 65-69, 1999.

[126] R. M. Sievert, Die Intensitätsverteilung der primären $\gamma$-strahlung in der Nähe medizinischer Radiumpräparate. Acta Radiol., vol. 1, pp. 89-128, 1921.

[127] I. MichieLI, Point kernel calculations of dose fields from line sources using expanded polynomial form of buildup factor data: generalized secant integral series representations. Radiat. Phis. Chem., vol. 51, pp. 121-128, 1998.

[128] R. G. Siddall, Accurate evaluation of radiative direct-exchange areas for rectangular geometries. En: Proceedings of the 8th international heat transfer conference, vol. 2, pp. 751-756, 1986.

[129] B. Ström, A simplified dynamic model of reheating furnaces. Tesis Doctoral. Estocolmo: Kungliga Tekniska Högskolan, 1979.

[130] I. MichieLI, Some properties of generalized secant integrals: extended definition and recurrence relations. Radiat. Phis. Chem., vol. 60, pp. 551-554, 2001.

[131] C.-J. Hsu, Shape factor equation for radiant heat transfer between two arbitrary sizes of rectangular planes. Can. J. Chem. Eng., vol. 45, pp. 58-60, 1967.

[132] J. R. Howell, A catalog of radiation configuration factors. Nueva York: McGrawHill, 1982.

[133] H. C. Hottel, Radiant heat transmission. En: Heat transmission, 3. ${ }^{\mathrm{a}}$ ed., W. H. McAdams (Ed.). Nueva York: McGraw-Hill, 1954.

[134] W. W. YUEn, The multiple absorption coefficient zonal method (MACZM), an efficient computational approach for the analysis of radiative heat transfer in multidimensional inhomogeneous nongray media. Numer. Heat Transfer, vol. B49, pp. 89-103, 2006. 



\section{Nomenclatura}

\section{Símbolos latinos}

a semieje del elipsoide de contacto entre sólidos elásticos (m)

$a_{k}(T) \quad$ peso de la WSGG evaluado a la temperatura $T$

$A \quad$ área de una superficie $\left(\mathrm{m}^{2}\right)$

$b \quad$ semieje del elipsoide de contacto entre sólidos elásticos (m)

$b_{0} \quad$ coeficiente de un polinomio

$b_{1} \quad$ coeficiente de un polinomio

$b_{2} \quad$ coeficiente de un polinomio

$c_{p b} \quad$ capacidad calorífica media de las baldosas $\left(\mathrm{J} \mathrm{kg}^{-1} \mathrm{~K}^{-1}\right)$

$c_{p r} \quad$ capacidad calorífica media de los rodillos $\left(\mathrm{J} \mathrm{kg}^{-1} \mathrm{~K}^{-1}\right)$

$d \quad$ distancia $(\mathrm{m})$

e residuo del sistema de balances de energía linealizados (W)

$E_{i} \quad$ poder emisivo del cuerpo negro a la temperatura $T_{i}\left(\mathrm{~W} \mathrm{~m}^{-2}\right)$

$g \quad$ aceleración de la gravedad $\left(9,81 \mathrm{~m} \mathrm{~s}^{-2}\right)$

$\overline{g_{i} g_{j}} \quad$ área de intercambio directo entre volúmenes de gas $\left(\mathrm{m}^{2}\right)$

[g. matriz de áreas de intercambio directo entre volúmenes de gas $\left(\mathrm{m}^{2}\right)$

$\overline{G_{i} G_{j}} \quad$ área de intercambio total entre volúmenes de gas $\left(\mathrm{m}^{2}\right)$

[GG $]$ matriz de áreas de intercambio total entre volúmenes de gas $\left(\mathrm{m}^{2}\right)$

$\overrightarrow{G_{i} G_{j}} \quad$ área de flujo dirigido desde un volumen de gas hasta otro volumen de gas $\left(\mathrm{m}^{2}\right)$

[ $\overrightarrow{\mathbf{G G}}] \quad$ matriz de áreas de flujo dirigido desde volúmenes de gas hasta otros volúmenes de gas $\left(\mathrm{m}^{2}\right)$

$\overline{g_{i} s_{j}} \quad$ área de intercambio directo entre un volumen de gas y una superficie $\left(\mathrm{m}^{2}\right)$

$[\overline{\mathrm{gs}}] \quad$ matriz de áreas de intercambio directo entre volúmenes de gas y superficies $\left(\mathrm{m}^{2}\right)$ 
$\overline{G_{i} S_{j}} \quad$ área de intercambio total entre volúmenes de gas y superficies $\left(\mathrm{m}^{2}\right)$

[GS matriz de áreas de intercambio total entre volúmenes de gas y superficies $\left(\mathrm{m}^{2}\right)$

$\overrightarrow{G_{i} S_{j}} \quad$ área de flujo dirigido desde un volumen de gas hasta una superficie $\left(\mathrm{m}^{2}\right)$

[GS matriz de áreas de flujo dirigido desde volúmenes de gas hasta superficies $\left(\mathrm{m}^{2}\right)$

$h_{C} \quad$ coeficiente individual de transferencia de energía por convección $\left(\mathrm{W} \mathrm{m}^{-2} \mathrm{~K}^{-1}\right)$

$h_{R} \quad$ coeficiente individual de transferencia de energía por radiación $\left(\mathrm{W} \mathrm{m}^{-2} \mathrm{~K}^{-1}\right)$

$h_{x} \quad$ coeficiente local de transmisión de calor por contacto entre las baldosas y los rodillos $\left(\mathrm{W} \mathrm{m}^{-2} \mathrm{~K}^{-1}\right)$

$K \quad$ coeficiente de absorción de un gas gris $\left(\mathrm{m}^{-1}\right)$

$\ell \quad \quad$ espesor de una pared $(\mathrm{m})$

$\ell_{b}^{(j)} \quad$ espesor de una de las capas en las que se dividen las baldosas (m)

L longitud característica (m)

$L_{1} \quad$ longitud de un sector del horno (m)

$L_{2} \quad$ anchura útil del horno $(\mathrm{m})$

$L_{3} \quad$ distancia entre las baldosas y la bóveda o la solera $(\mathrm{m})$

m número de zonas de superficie

$\dot{m}^{(j)} \quad$ caudal másico de baldosas crudas dividido por el número de capas en las que éstas se dividen $\left(\mathrm{kg} \mathrm{s}^{-1}\right)$

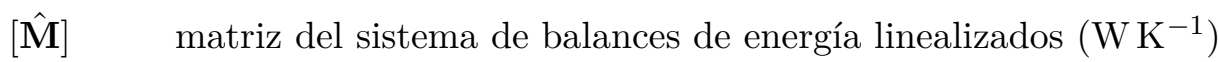

n número de zonas de volumen

$\mathrm{Nu} \quad$ módulo de Nusselt

presión $(\mathrm{Pa})$

$p_{R} \quad$ suma de las presiones parciales de $\mathrm{H}_{2} \mathrm{O}$ y de $\mathrm{CO}_{2}(\mathrm{~Pa})$

Pr módulo de Prandtl

q número de gases grises en la WSGG

$\dot{Q} \quad$ flujo de calor $(\mathrm{W})$

$\dot{Q}_{i \rightarrow j} \quad$ flujo de energía radiante que abandona la superficie $A_{i}$ (o el volumen $V_{i}$ ) y alcanza directamente a la superficie $A_{j}$ (o al volumen $V_{j}$ ) (W) 
$\{\hat{\mathbf{Q}}\} \quad$ vector de términos independientes del sistema de balances de energía linealizados $(\mathrm{W})$

$r \quad$ distancia entre dos puntos $(\mathrm{m})$

$R \quad$ radio de los rodillos $(\mathrm{m})$

[R] matriz que surge en el cálculo de las TEA $\left(\mathrm{m}^{-2}\right)$

Ra módulo de Rayleigh

$\overline{s_{i} g_{j}} \quad$ área de intercambio directo entre una superficie y un volumen de gas $\left(\mathrm{m}^{2}\right)$

[sg] matriz de áreas de intercambio directo entre superficies y volúmenes de gas $\left(\mathrm{m}^{2}\right)$

$\overline{S_{i} G_{j}} \quad$ área de intercambio total entre una superficie y un volumen de gas $\left(\mathrm{m}^{2}\right)$

[SG $]$ matriz de áreas de intercambio total entre superficies y volúmenes de gas $\left(\mathrm{m}^{2}\right)$

$\overrightarrow{S_{i} G_{j}} \quad$ área de flujo dirigido desde una superficie hasta un volumen de gas $\left(\mathrm{m}^{2}\right)$

$[\overrightarrow{\mathbf{S G}}] \quad$ matriz de áreas de flujo dirigido desde superficies hasta volúmenes de gas $\left(\mathrm{m}^{2}\right)$

$\overline{s_{i} s_{j}} \quad$ área de intercambio directo entre superficies $\left(\mathrm{m}^{2}\right)$

$[\overline{\mathbf{s s}}] \quad$ matriz de áreas de intercambio directo entre superficies $\left(\mathrm{m}^{2}\right)$

$\overline{S_{i} S_{j}} \quad$ área de intercambio total entre superficies $\left(\mathrm{m}^{2}\right)$

$[\overline{\mathbf{S S}}] \quad$ matriz de áreas de intercambio total entre superficies $\left(\mathrm{m}^{2}\right)$

$\overrightarrow{S_{i} S_{j}} \quad$ área de flujo dirigido desde una supercie hasta otra superficie $\left(\mathrm{m}^{2}\right)$

$[\overrightarrow{\mathbf{S S}}] \quad$ matriz de áreas de flujo dirigido desde superficies hasta otras superficies $\left(\mathrm{m}^{2}\right)$

T temperatura $(\mathrm{K})$

$T_{a} \quad$ temperatura del aire comburente $(\mathrm{K})$

$T_{b} \quad$ temperatura de las baldosas $(\mathrm{K})$

$T_{e} \quad$ temperatura del aire de enfriamiento incorporado al calentamiento $(\mathrm{K})$

$T_{g} \quad$ temperatura de un volumen de gas $(\mathrm{K})$

$T_{i} \quad$ temperatura de una superficie interior del horno $(\mathrm{K})$

$T_{\text {máx }} \quad$ temperatura máxima de cocción $(\mathrm{K})$

$T_{o} \quad$ temperatura de una superficie exterior del horno (K)

$T_{\text {ref }} \quad$ temperatura de referencia $(298,15 \mathrm{~K})$ 
$T_{\infty} \quad$ temperatura ambiente $(\mathrm{K})$

$\{\mathbf{T}\} \quad$ vector de temperaturas de las zonas $(\mathrm{K})$

$\{\hat{\mathbf{T}}\} \quad$ vector de temperaturas de las zonas estimadas (K)

$u \quad$ velocidad de avance de las baldosas $\left(\mathrm{ms}^{-1}\right)$

$u_{g} \quad$ velocidad media de circulación de los gases $\left(\mathrm{m} \mathrm{s}^{-1}\right)$

$U \quad$ coeficiente global de transmisión de calor $\left(\mathrm{W} \mathrm{m}^{-2} \mathrm{~K}^{-1}\right)$

$V \quad$ volumen $\left(\mathrm{m}^{3}\right)$

$V_{\mathrm{CO}_{2}} \quad$ volumen de $\mathrm{CO}_{2}$ generado en la combustión de una unidad de volumen de combustible

$V_{\mathrm{H}_{2} \mathrm{O}} \quad$ volumen de $\mathrm{H}_{2} \mathrm{O}$ generado en la combustión de una unidad de volumen de combustible

$V_{\mathrm{O}_{2} \text { mín }} \quad$ volumen mínimo de $\mathrm{O}_{2}$ requerido para completar la combustión de una unidad de volumen de combustible

$\dot{V}_{a} \quad$ caudal volumétrico de aire comburente en condiciones normales $\left(\mathrm{m}^{3} \mathrm{~s}^{-1}\right)$

$\dot{V}_{c} \quad$ caudal volumétrico de combustible en condiciones normales $\left(\mathrm{m}^{3} \mathrm{~s}^{-1}\right)$

$\dot{V}_{d} \quad$ caudal volumétrico de productos de descomposición de las baldosas en condiciones normales $\left(\mathrm{m}^{3} \mathrm{~s}^{-1}\right)$

$\dot{V}_{e} \quad$ caudal volumétrico de aire de enfriamiento incorporado al calentamiento en condiciones normales $\left(\mathrm{m}^{3} \mathrm{~s}^{-1}\right)$

$\dot{V}_{g} \quad$ caudal volumétrico de humos en condiciones normales $\left(\mathrm{m}^{3} \mathrm{~s}^{-1}\right)$

$\dot{V}_{k} \quad$ caudal volumétrico de la especie $k$ en condiciones normales $\left(\mathrm{m}^{3} \mathrm{~s}^{-1}\right)$

$w_{k} \quad$ fracción másica inicial del componente de referencia de la $k$-ésima reacción química

$x \quad$ coordenada espacial $(\mathrm{m})$

$x_{k} \quad$ fracción volumétrica de la especie $k$

$x_{\mathrm{O}_{2} a} \quad$ fracción volumétrica de $\mathrm{O}_{2}$ en el aire comburente

y coordenada espacial $(\mathrm{m})$

$z \quad$ coordenada espacial $(\mathrm{m})$

$\overrightarrow{Z_{i} Z_{j}} \quad$ área de flujo dirigido generalizada $\left(\mathrm{m}^{2}\right)$

$[\overrightarrow{\mathbf{Z Z}}] \quad$ matriz de áreas de flujo dirigido generalizadas $\left(\mathrm{m}^{2}\right)$ 


\section{Símbolos griegos}

$\alpha \quad$ factor de relajación

$\alpha_{a} \quad$ difusividad térmica del aire $\left(\mathrm{m}^{2} \mathrm{~s}^{-1}\right)$

$\alpha_{b} \quad$ difusividad térmica aparente de las baldosas $\left(\mathrm{m}^{2} \mathrm{~s}^{-1}\right)$

$\beta \quad$ coeficiente de expansión cúbica $\left(\mathrm{K}^{-1}\right)$

$\delta \quad$ separación entre los ejes de los rodillos $(\mathrm{m})$

$\Delta H_{a}^{\ominus}(T)$ variación en la entalpía específica del aire comburente en el estado base al pasar de la temperatura de referencia a la temperatura $T\left(\mathrm{~J} \mathrm{~m}^{-3}\right)$

$\Delta H_{d}^{\ominus}(T)$ variación en la entalpía específica de los productos de descomposición de las baldosas en el estado base al pasar de la temperatura de referencia a la temperatura $T\left(\mathrm{~J} \mathrm{~m}^{-3}\right)$

$\Delta H_{e}^{\ominus}(T)$ variación en la entalpía específica del aire de enfriamiento incorporado al calentamiento en el estado base al pasar de la temperatura de referencia a la temperatura $T\left(\mathrm{~J} \mathrm{~m}^{-3}\right)$

$\Delta H_{g}^{\ominus}(T)$ variación en la entalpía específica de los humos en el estado base al pasar de la temperatura de referencia a la temperatura $T\left(\mathrm{~J} \mathrm{~m}^{-3}\right)$

$\Delta H_{k}^{\ominus}(T)$ variación en la entalpía específica de la especie $k$ en el estado base al pasar de la temperatura de referencia a la temperatura $T\left(\mathrm{~J} \mathrm{~m}^{-3}\right)$

$\Delta_{r} H_{c}^{\ominus} \quad$ calor de la reacción de combustión del gas natural en el estado base y a la temperatura de referencia $\left(\mathrm{J} \mathrm{m}^{-3}\right)$

$\Delta_{r} H_{k}^{\ominus} \quad$ calor de reacción de la $k$-ésima reacción química en el estado base y a la temperatura de referencia $\left(\mathrm{J} \mathrm{kg}^{-1}\right.$ o $\left.\mathrm{J} \mathrm{m}^{-3}\right)$

$\varepsilon \quad$ emisividad

$\varepsilon_{R} \quad$ emisividad resultante

$\theta_{i} \quad$ ángulo formado entre el vector normal a la superficie $A_{i}$ y el vector que une dos puntos de las superficies $A_{i}$ y $A_{j}$

$\Theta_{k} \quad$ temperatura adimensional definida en la ecuación (1.17)

$\kappa_{k} \quad$ coeficiente de absorción del gas gris $k\left(\mathrm{~Pa}^{-1} \mathrm{~m}^{-1}\right)$

$\lambda \quad$ conductividad térmica $\left(\mathrm{W} \mathrm{m}^{-1} \mathrm{~K}^{-1}\right)$

$\lambda_{a} \quad$ conductividad térmica del aire $\left(\mathrm{W} \mathrm{m}^{-1} \mathrm{~K}^{-1}\right)$

$\lambda_{b} \quad$ conductividad térmica de las baldosas $\left(\mathrm{W} \mathrm{m}^{-1} \mathrm{~K}^{-1}\right)$ 
$\lambda_{r} \quad$ conductividad térmica de los rodillos $\left(\mathrm{W} \mathrm{m}^{-1} \mathrm{~K}^{-1}\right)$

$\Lambda \quad$ recorrido libre medio molecular modificado $(\mathrm{m})$

$\nu_{a} \quad$ viscosidad cinemática del aire $\left(\mathrm{m}^{2} \mathrm{~s}^{-1}\right)$

$\xi_{k} \quad$ grado de avance de la $k$-ésima reacción química referido al componente de referencia

$\varrho \quad$ reflectancia

$\rho_{b} \quad$ densidad aparente de las baldosas $\left(\mathrm{kg} \mathrm{m}^{-3}\right)$

$\rho_{k} \quad$ densidad de la especie $k$ en condiciones normales $\left(\mathrm{kg} \mathrm{m}^{-3}\right)$

$\rho_{r} \quad$ densidad aparente de los rodillos $\left(\mathrm{kg} \mathrm{m}^{-3}\right)$

$\sigma$

constante de Stefan-Boltzmann $\left(5,67032 \cdot 10^{-8} \mathrm{~W} \mathrm{~m}^{-2} \mathrm{~K}^{-4}\right)$

$\tau \quad$ duración del ciclo de cocción (s) 


\section{Acrónimos}

DEA direct exchange area

DFA directed flux area

GEI gas de efecto invernadero

IUPAC International Union of Pure and Applied Chemistry

NIST National Institute of Standards and Technology

PCI poder calorífico inferior

PCS poder calorífico superior

TEA total exchange area

WSGG weighted sum of grey gases 